SANDIA REPORT

SAND98-2050

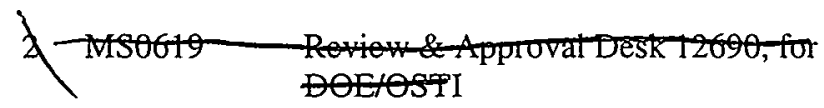

Unlimited Release

Printed September 1998

\title{
Data Qualíty Management Plan (DQMP) for the Alternative Landfill Cover Demonstrátion Project
} $\sqrt{2}$

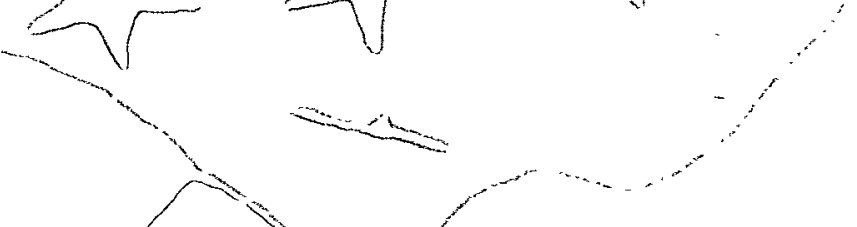

A 033

OSTI

Stephen F. Dwyer, Jesus Lopez, and Richard Aguilar

Prepared by

Sandia National Laboratories

Albuquerque, New Mexico - $87-185$ and Livermore, California 94550

Sandia is a multiprogram laboratory operated by Sandia Corporation, a Lockheed Martin Company, for the United States Department of

Energy under Contract DE-AC04-94AL85000.

Approved for public release; further dissemination unlimited. 
Issued by Sandia National Laboratories, operated for the United States Department of Energy by Sandia Corporation.

NOTICE: This report was prepared as an account of work sponsored by an agency of the United States Government. Neither the United States Government, nor any agency thereof, nor any of their employees, nor any of their contractors, subcontractors, or their employees, make any warranty, express or implied, or assume any legal liability or responsibility for the accuracy, completeness, or usefulness of any information, apparatus, product, or process disclosed, or represent that its use would not infringe privately owned rights. Reference herein to any specific commercial product, process, or service by trade name, trademark, manufacturer, or otherwise, does not necessarily constitute or imply its endorsement, recommendation, or favoring by the United States Government, any agency thereof, or any of their contractors or subcontractors. The views and opinions expressed herein do not necessarily state or reflect those of the United States Government, any agency thereof, or any of their contractors.

Printed in the United States of America. This report has been reproduced directly from the best available copy.

Available to DOE and DOE contractors from

Office of Scientific and Technical Information

P.O. Box 62

Oak Ridge, TN 37831

Prices available from (703) 605-6000

Web site: http://www.ntis.gov/ordering.htm

Available to the public from

National Technical Information Service

U.S. Department of Commerce

5285 Port Royal Rd

Springfield, VA 22161

NTIS price codes

Printed copy: A17

Microfiche copy: A01

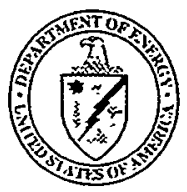




\section{DISCLAIMER}

Portions of this document may be illegible in electronic image products. Images are produced from the best available original document. 
SAND98-2050

Unlimited Release

Printed September 1998

\title{
Data Quality Management Plan (DQMP) for the Alternative Landfill Cover Demonstration Project
}

TTP No.: AL27SS27

\author{
Prepared for: \\ U.S. Department of Energy, Office of Science and Technology \\ Subsurface Contaminants Focus Area
}

\author{
Stephen F. Dwyer and Jesus Lopez \\ Environmental Restoration Technology Department \\ Sandia National Laboratories \\ P.O. Box 5800 \\ Albuquerque, NM 87185-0719 \\ Richard Aguilar \\ YMP Performance Assessment \\ Sandia National Laboratories \\ P.O. Box 5800 \\ Albuquerque, NM 87185-0778
}

\begin{abstract}
The Alternative Landfill Cover Demonstration (ALCD) is a large-scale field test that compares the effectiveness of various landfill cover designs in dry environments. More specifically, the demonstration compares the performance of four alternative designs specifically geared for drier environments to the performance of two cover designs endorsed by the Environmental Protection Agency (EPA) for nationwide use. For a thorough comparison, data concerning the weather, soil moisture status, surface runoff, lateral drainage, and percolation through the covers must be collected. A complex array of instrumentation is in place to continually collect this data. The system is completely automated. There are manual backup systems in place to verify the accuracy of the primary automated system as well as serve as backup in case of failure in any part
\end{abstract}


of the primary system. This document gives a detailed description of the project including its scope, test configuration, test plot designs, construction quality assurance, material quality assurance, instrumentation/monitoring equipment and procedures data analysis and interpretation, data quality assurance, environmental safety and health requirements, and expected benefits from the ALCD project. All pertinent information is also included. 


\section{CONTENTS}

\section{Page}

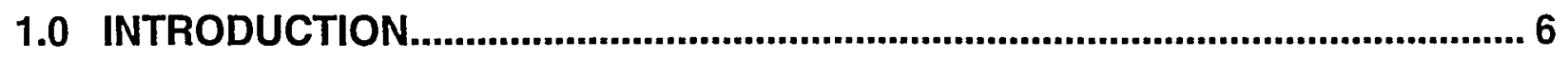

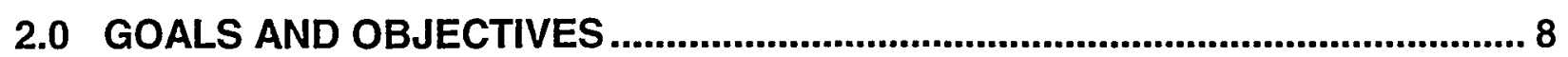

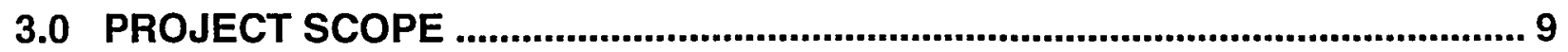

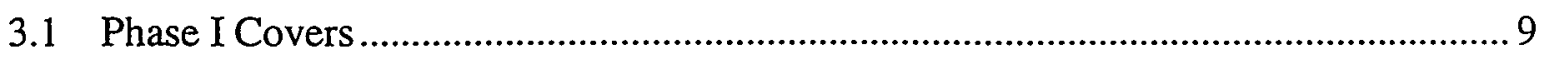

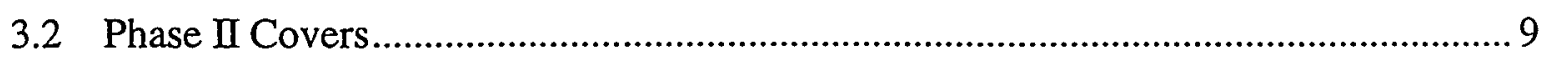

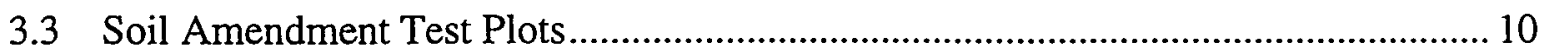

3.4 Evaluation of Alternative Cover Design Performance.............................................. 11

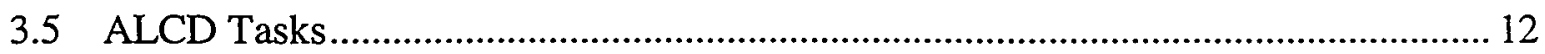

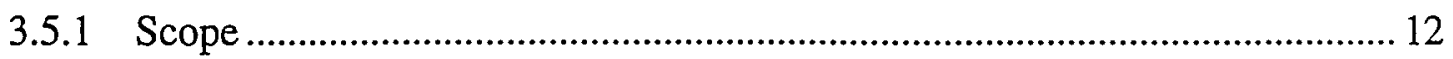

3.5.2 Site Acquisition, Characterization, and Permitting ..................................... 12

3.5.3 Design of Landfill Covers and Soil Amendment Plots ................................... 13

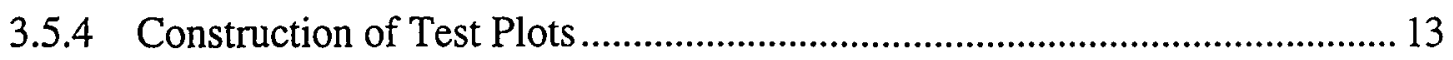

3.5.5 Monitoring of Test Plots .......................................................................... 13

3.5.6 Documentation of Cover Design Performance ............................................ 13

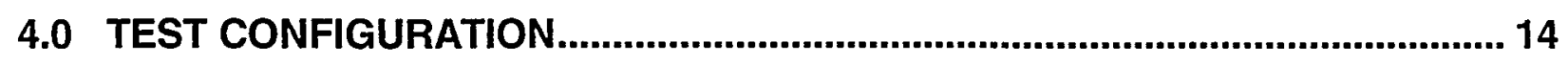

5.0 DESIGN/CONSTRUCTION OF TEST COVERS .............................................. 15

5.1 Baseline Cover Designs.................................................................................... 15

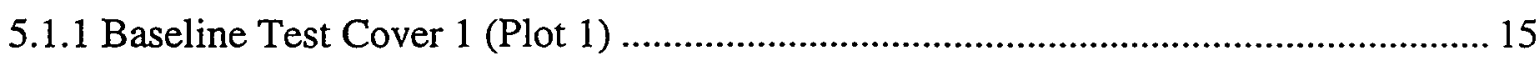

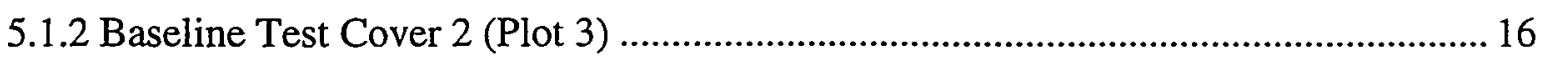

5.2 Alternative Cover Designs ...........................................................................

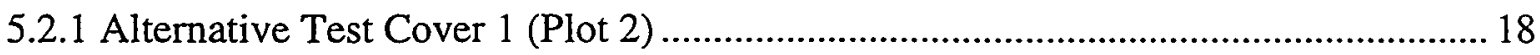

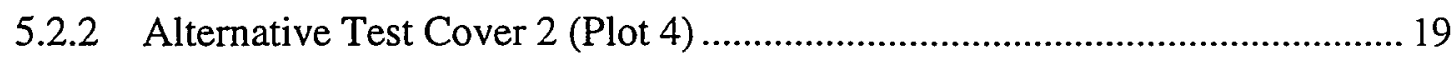

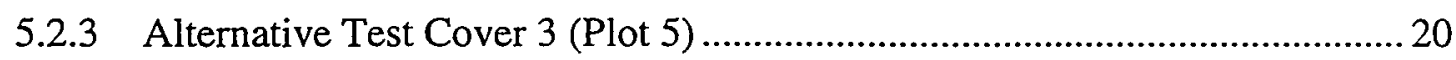

5.2.4 Alternative Test Cover 4 (Plot 6) ................................................................. 21

6.0 CONSTRUCTON QUALITY ASSURANCE .......................................................... 22

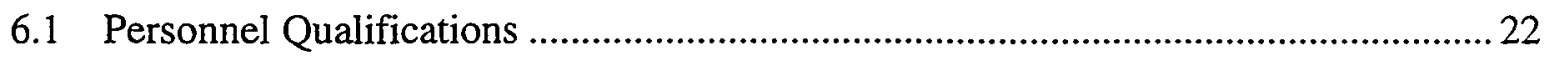

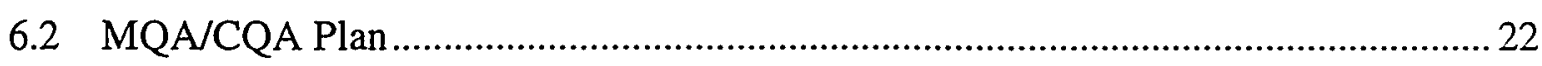

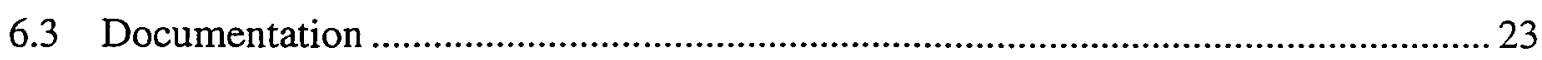

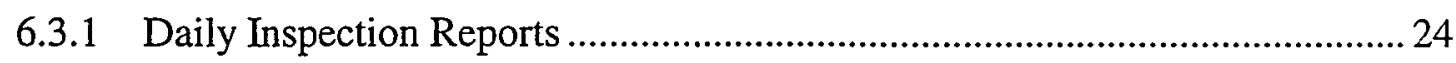

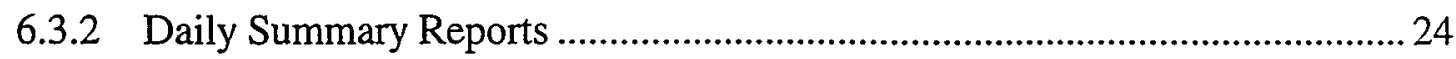

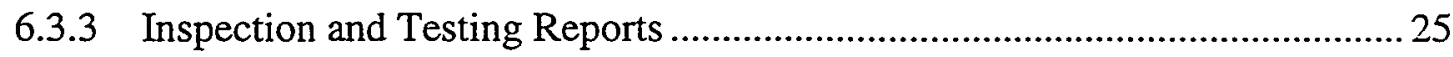

6.3.4 Problem Identification \& Corrective Measures Reports .................................... 25

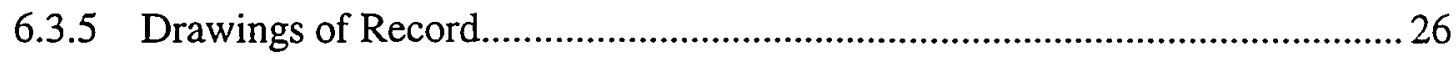




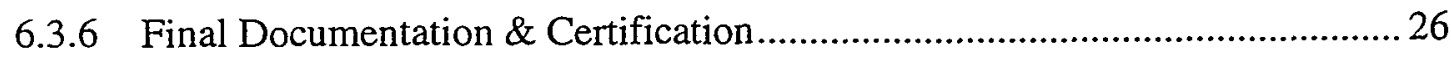

6.3.7 Document Control ................................................................................. 26

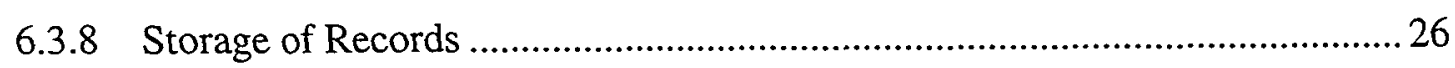

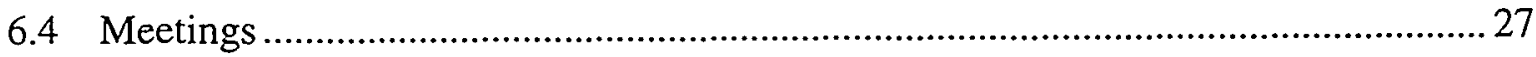

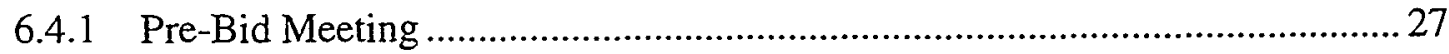

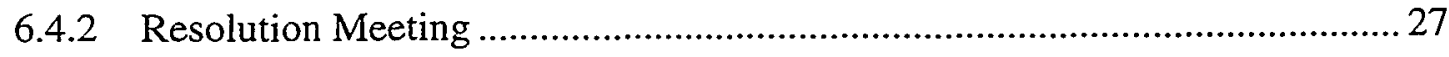

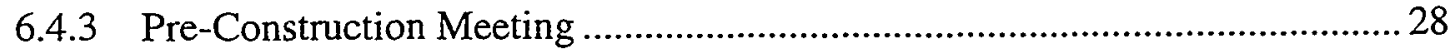

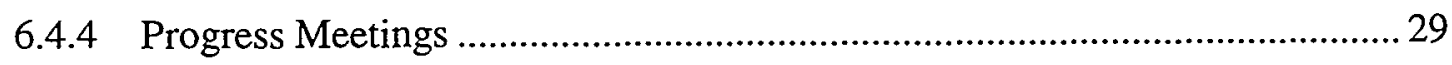

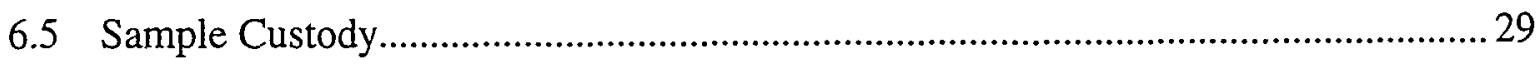

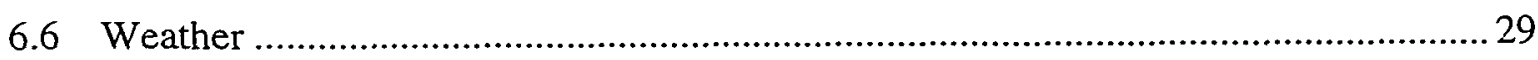

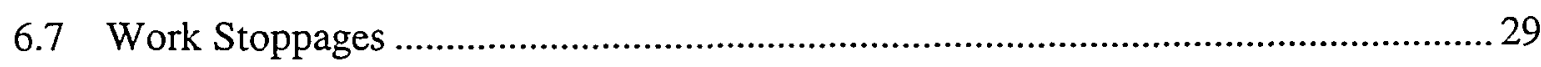

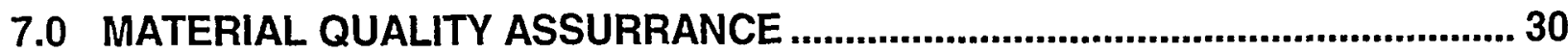

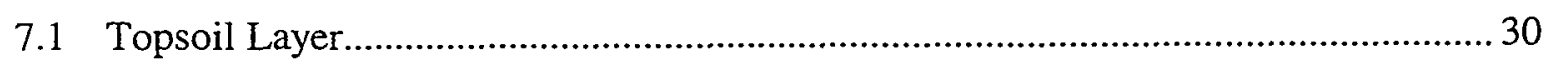

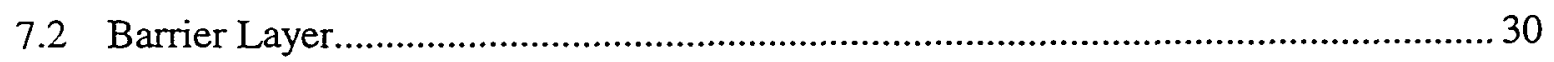

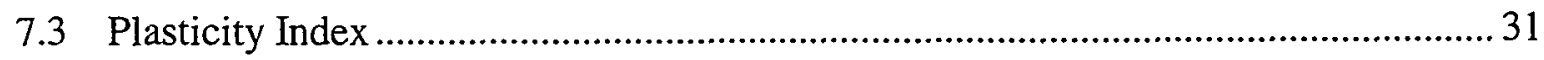

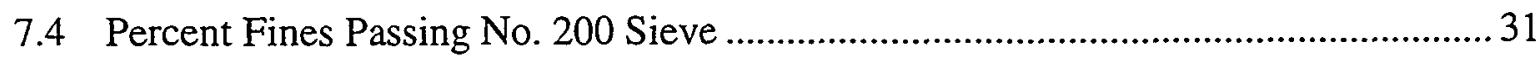

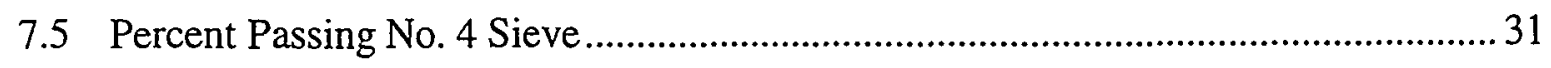

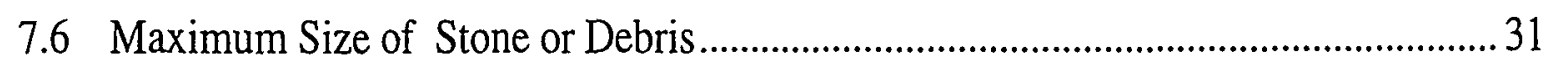

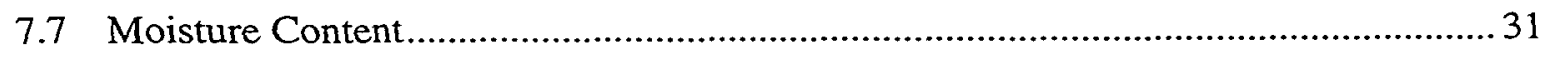

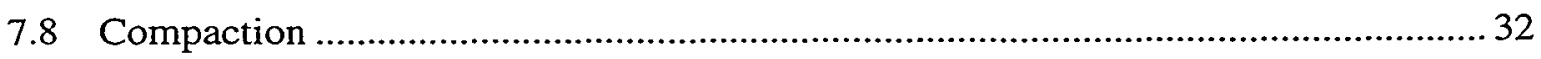

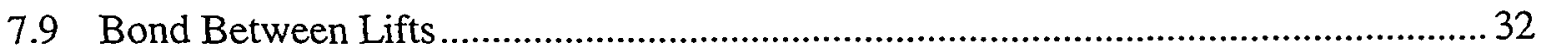

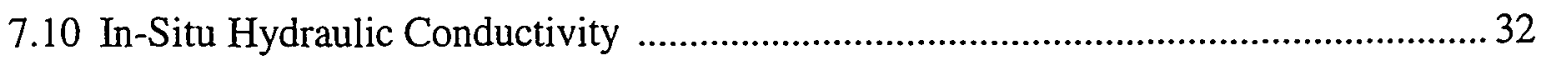

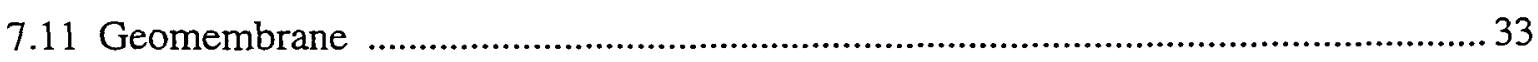

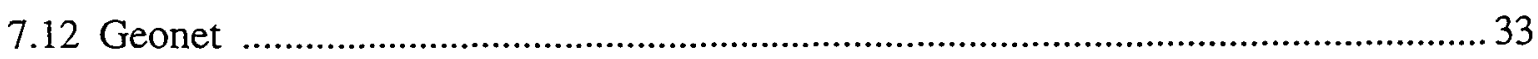

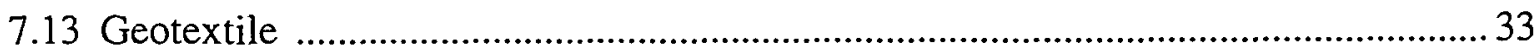

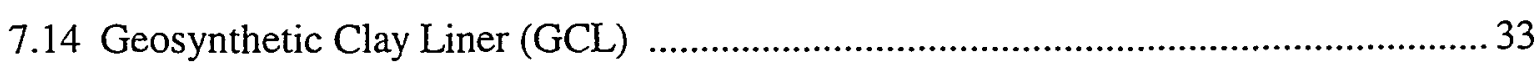

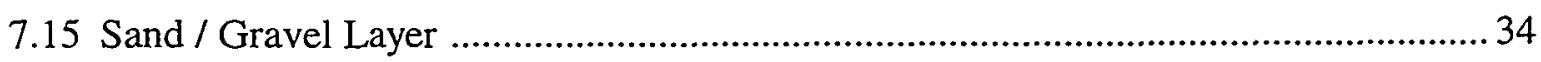

8.0 INSTRUMENTATION/MONITORING EQUIPMENT \& PROCEDURES ............... 41

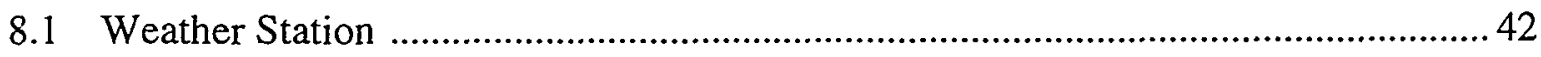

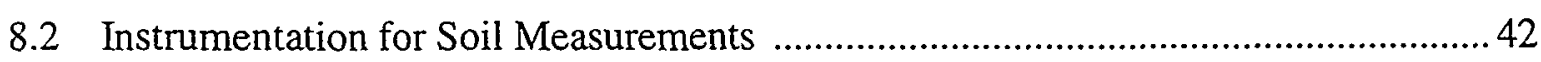

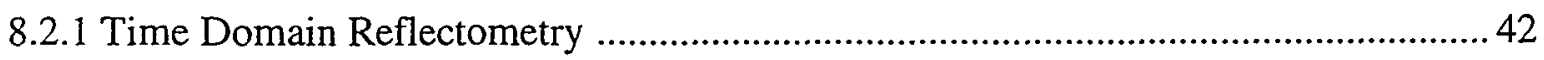

8.2.2 TDR Calibration for Phase I Cover Soil Materials ................................................... 45

8.2.3 TDR Calibration for Phase II Cover Soil Materials .................................................... 46

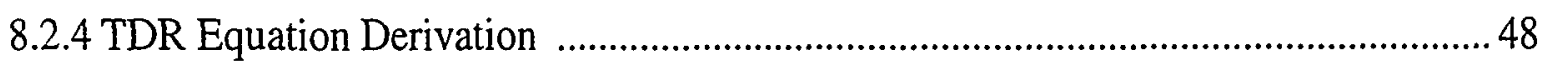

8.3 Surface Runoff, Percolation, and Interflow Measurements ........................................49

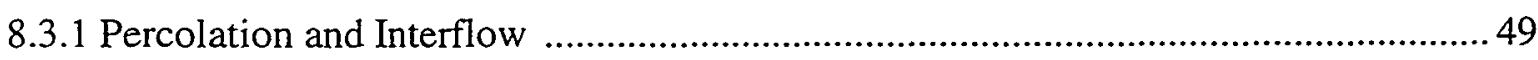

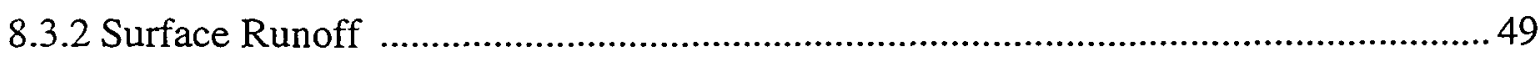


8.4 Data Collection and Organization .................................................................... 51

8.4.1 Stage 1: Data Collection - Data Logger ............................................................. 51

8.4.2 Stage 2: Data Collection - Central Computer ............................................ 53

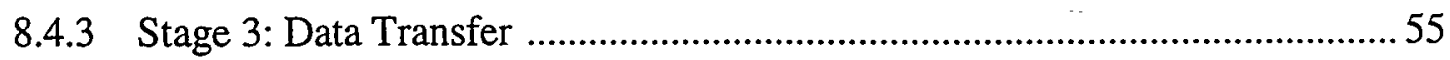

9.0 DATA ANALYSIS AND INTERPRETATION .................................................. 56

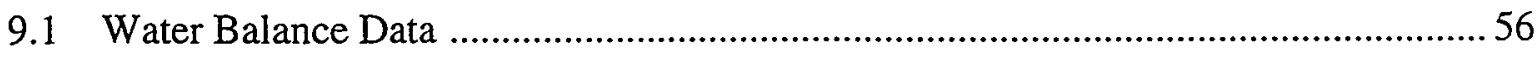

9.2 Comparison with Predictive Models ............................................................... 57

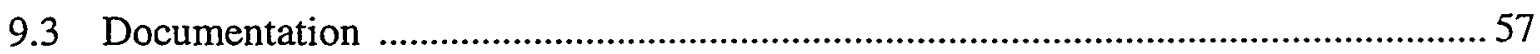

10.0 DATA QUALITY ASSURANCE .............................................................. 58

11.0 ENVIRONMENTAL SAFETY AND HEALTH REQUIREMENTS ........................59

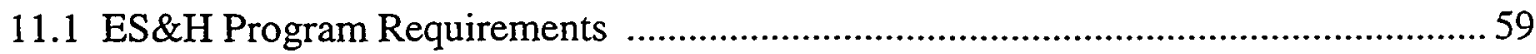

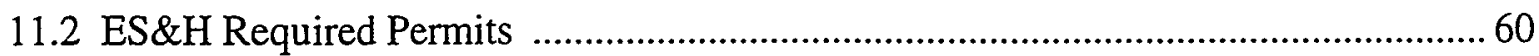

12.0 EXPECTED BENEFITS FROM THE ALCD PROJECT ................................... 60

13.0 LITERATURE CITED ............................................................................ 61

ATTACHMENTS:

A Soil Amendment Study Implementation \& Procedures ......................................A-1

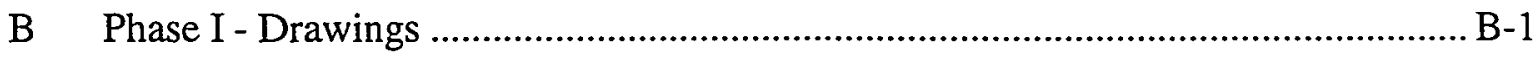

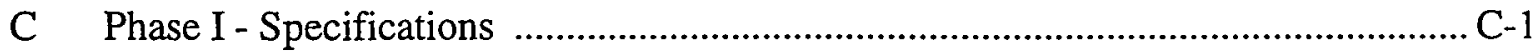

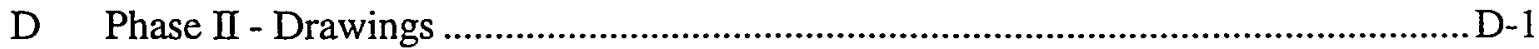

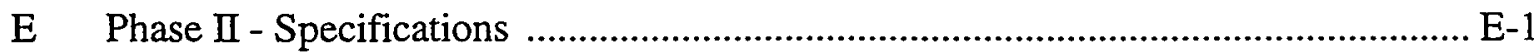

F TDR Calibration - Volumetric Moisture Content ................................................

G TDR Calibration - Polynomial Derivation ........................................................

H Surface Runoff System Calibration................................................................. 


\subsection{INTRODUCTION}

The Alternative Landfill Cover Demonstration (ALCD) is a large-scale field test designed to compare and contrast the performance of various landfill cover designs in an arid environment. A total of six landfill covers were constructed for testing. The cover designs include two baseline covers, a soil cover that meets minimum requirements set forth by the Environmental Protection Agency's (EPA) Resource Conservation and Recovery Act (RCRA) Subtitle ' $D$ ' governing municipal landfills and a traditional EPA/RCRA Subtitle ' $C$ ' Clay Cover principally used for hazardous waste landfills. Four additional "custom design" alternative covers to emphasize a dry environment were also constructed and will be tested. Precipitation and several soil water parameters, including the moisture content at various depths, runoff, and percolation below the cover, will be continuously monitored for all six landfill covers. The demonstration project was implemented in two phases. Phase I, consisted of the construction in FY95 of three separate covers installed side-by-side (the two regulatory standard covers described above and one alternative cover). Phase II construction, consisting of three additional alternative covers (also installed side-by-side), was implemented and completed in FY96. Field data acquisition began immediately upon the completion of the Phase II landfill covers. Predicted water balances generated through computer modeling will be compared with estimates based upon field measurements.

As an extension of the landfill cover study, a multi-plot soil amendment study was established to evaluate the effectiveness of various amendments aimed at improving soil conditions for plant establishment and growth. Improved topsoil conditions and increased vegetation establishment and growth result in greater evapotranspiration, reduced percolation, and therefore greater isolation of near-surface wastes and contaminants. The soil amendment study will focus on testing the performance of various methods for enhancing the establishment of native grasses and forbs, including a component of mesic plants (those which are most biologically active during the cool spring months when the potential for subsurface leaching and contaminant transport is greatest). Study plot soils were analyzed prior to and after the amendment of various organic materials to test for changes in soil organic matter and plant nutrient availability. The plots were seeded similarly to the landfill covers with native rangeland grassland plant species. Vegetation analyses will include periodic measurement of plant establishment and growth as a function of the various treatments. It is hoped that the study results will provide a basis for the establishment of enhanced revegetation guidelines for landfill covers in arid and semiarid regions.

In 1988, the U.S. EPA identified the need for research into improved landfills and alternative landfill cover designs because the traditional covers were deemed inadequate under certain circumstances (EPA, 1988). The EPA evaluated 163 randomly chosen landfills across the country to arrive at this conclusion. Of these 163 landfills, 146 sites had experienced groundwater contamination with severity ranging from elevated contaminant constituents in "onsite" groundwater to severe contamination of major aquifers and productive well fields. Thirtyfive of the sites had experienced severe contamination which required immediate drastic action; this included changing the water supply because Federal and/or State drinking water standards had been compromised. Seventy-three of the sites reported surface water contamination and, thirteen sites reported severe ecological impacts such as fish kills and short-term or chronic long- 
term damage to the health of aquatic flora and/or fauna. Many of these sites displayed multiple adverse ecological symptoms. Virtually all parts of the nation have experienced water contamination to some degree from landfill waste.

Landfill integrity depends on its ability to contain waste. The landfill cover is designed to minimize percolation of water into the underlying waste and thus minimize the generation of leachate. Arguably, the cover is the single most important component of a landfill. Many existing landfills were poorly sited and/or have no liners to isolate the waste within. Under these circumstances, an appropriately designed and engineered cover would play a major role in the continued isolation of the waste.

Based on the 1988 EPA study, there is an obvious need for improved landfill technology, particularly with cover design. The EPA Guidance Document for Design and Construction of RCRA/CERCLA Covers (EPA, 1991) states the following: "In arid regions, a barrier layer composed of clay (natural soil) and a geomembrane is not very effective. Since the soil is compacted 'wet of optimum', the layer will dry and crack'. The ALCD project is directly addressing these concerns by demonstrating and testing alternative covers for arid regions that improve on existing mandated technologies.

Development of an improved technology, however, does not guarantee that it will be implemented. A key ingredient to the effective transfer of new remediation technologies to user organizations is acceptance or "buy-in" by the relevant regulatory authorities. A University of North Dakota study (Wentz, 1989) on the criteria considered for selecting a hazardous waste management technology revealed that the established regulatory guidelines for the particular problem(s) is the criteria most often considered. The technologic merits of the various technologies available for dealing with the problem(s) are considered last. The study revealed the following ranking of criteria considered for the selection of a waste management technology: (1) government regulations, (2) economics, (3) public relations, and (4) process/technology.

Recognizing that promoting the acceptance of alternative landfill construction technologies is a difficult task, the ALCD project involved industry experts and regulators from the beginning to provide input on the project's scope, design, and implementation.

Reviewers of this test plan included:

\section{Industry Experts:}

Dr. David Daniel

Dr. Tom Hakonson

Gerald Peace

- Dr. John Stormont
Geotechnical Engineer, Civil Engineering Department, University of Texas.

Ecologist \& Environmental Scientist, Risk Assessment

Department, Colorado State University.

Environmental Engineer, Environmental Restoration Group, Sandia National Laboratories.

Geomechanics Engineer, Department of Civil Engineering, University of New Mexico. 
Brian Dwyer

\section{Regulators:}

Robert Landreth

Nancy Morelock

Mercedes Vidan

Anthony Leverrock

Ram Ramanujan

Jeff Kelley

Jeff Denison

Matt McDermott

Richard Anderson

Marty Gray
Environmental Engineer, , Environmental Restoration

Technologies Department (Dept. 6131), Sandia National

Laboratories

\section{U.S. EPA, Cincinnati, OH \\ U.S. EPA, Region VI, Dallas, TX}

Solid Waste Section, Dept. of Environmental Quality - State of Arizona, Phoenix, AZ

Hazardous Waste Section, Dept. of Environmental Quality - State of Arizona, Phoenix, AZ

Department of Toxic Substances Control, State of California, Sacramento, CA

Dept. of Environmental Quality - State of Nebraska, Lincoln, NE

Bureau of Waste Management, Division of Environmental Protection - State of Nevada, Carson City, NV

South Dakota Department of Environment and Natural Resources, Pierre, SD

Industrial and Hazardous Waste Division, Texas Natural Resources and Conservation Commission, Austin, TX.

Division of Solid and Hazardous Waste, Dept. of Environmental

Quality - State of Utah, Salt Lake City, UT

\subsection{GOALS AND OBJECTIVES}

The goals of the ALCD Project are to field-test, compare, and document the performance of alternative landfill cover technologies (of various complexities and costs) for the interim stabilization and/or final closure of landfills in arid and semiarid environments. To accomplish these goals the following objectives must be fulfilled:

1) demonstrate the constructibility of cover design alternatives,

2) compare the overall costs of cover design alternatives,

3) measure the performance of the design alternatives over a five-year post-construction period using water balance and ancillary data (e.g., waste isolation and leachate minimization) as the primary evaluation criteria,

4) validate predictive models and decision tools for evaluating long-term performance of the cover designs, and

5) document the constructibility, technical performance, overall cost, and predictive model comparisons of the ALCD cover alternatives. 
The test covers are constructed side by side (3-meter buffers between the covers) to allow for direct comparison among the regulatory standard and alternative covers. The ALCD does not intend to highlight any one particular cover system, rather the focus of the project is to provide the necessary information (i.e., cost, construction, and performance data) for regulatory agencies to encourage their acceptance. It is our hope that the alternative covers be accepted by regulatory agencies, thus providing design engineers with more effective alternatives for waste isolation in arid and semiarid regions.

\subsection{PROJECT SCOPE}

The ALCD Project will evaluate several different landfill cover designs, representing a range of engineering complexities and installation costs. Due to funding constraints, the ALCD test covers were constructed in two separate phases. Phase I was built in FY95. The construction of Phase II occurred in FY96.

\subsection{Phase I Covers}

Three landfill covers were installed during Phase I of the project. These covers are referred to as resistive barriers because their design emphasizes an impermeable barrier with very low saturated hydraulic conductivity to minimize the infiltration and subsequent percolation of water through the cover. These include:

1) A simple soil cover adhering to minimum RCRA Subtitle ' $D$ ' regulations. (This cover is intended to serve as a baseline for solid waste landfills.)

2) The conventional EPA compacted clay cover adhering to minimum RCRA Subtitle ' $C$ ' regulations. (This cover is intended to serve as a baseline for hazardous waste landfills.)

3) Alternative Cover \#1 -- a replicate of the conventional Subtitle ' $\mathrm{C}$ ' compacted clay cover that substitutes a manufactured product (geosynthetic clay liner) for the compacted clay-barrier layer.

\subsection{Phase II Covers}

Three additional alternative covers were constructed in Phase II of the project deviate from the standard of providing low saturated hydraulic conductivity under saturated soil conditions as a measure of their adequacy. Instead, these covers are designed to minimize percolation under unsaturated soil conditions. The three covers include:

1) a capillary barrier,

2) an anisotropic barrier, and

3) an evapotranspiration (ET) cover. 


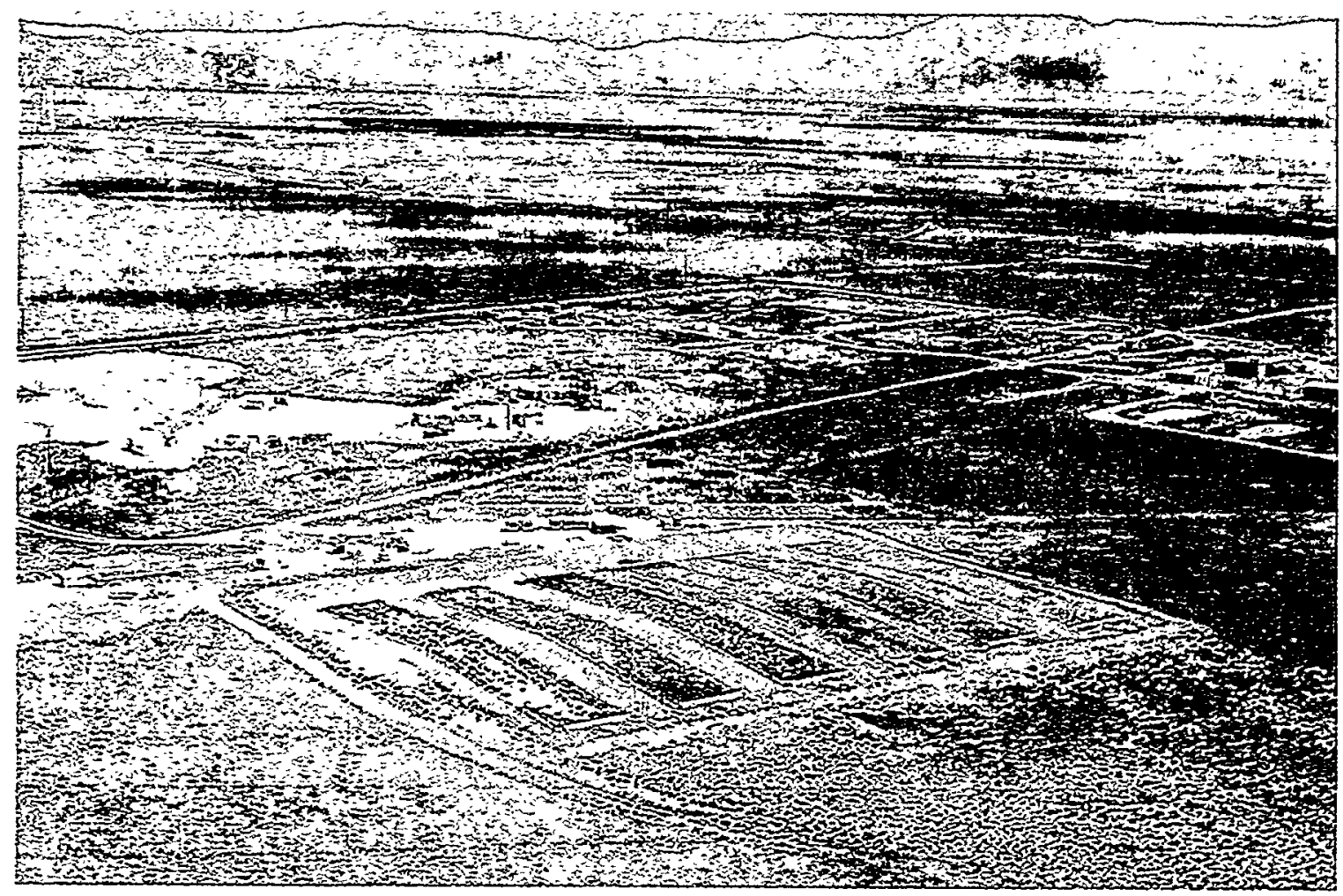

Figure 1. Aerial View of Alternative Landfill Cover Demonstration

\subsection{Soil Amendment Test Plots}

The soil amendment study has two primary technical objectives:

1) We will evaluate the changes in soil chemical, physical, and biological properties resulting from additions of organic amendments including biosolids compost, greenwaste compost, and dairy manure compost. The biosolids compost was derived primarily from treated municipal sewage sludge supplied by the City of Albuquerque. Green waste compost is derived from other organic materials, primarily plant material, separated from the City's solid waste prior to disposal and burial at the landfill. Soil properties analyzed will include those that affect plant establishment, production (growth), and long-term survival. Examples of chemical properties affecting plant growth are nitrogen, phosphorus, $\mathrm{pH}$, electrical conductivity (EC) and trace metals. Organic matter content will be measured because of its important relationship to water-holding capacity, soil structure, infiltration and permeability. Also being tested are the effects of cobble (5-8 cm diameter stones) mulching on infiltration, evaporation and soil-water storage. The effects of supplemental water (irrigation) added during the typically dry late spring to early summer months (May June) will also be investigated.

2) Quantify and correlate the differences in plant establishment and production under the various treatments tested and correlate these to soil properties (item \#1 above). 
Study plot soils were sampled and analyzed prior to the addition of the organic amendments. The organic amendments were rotar-tilled into the upper six inches of soil. The soil will be re-analyzed annually for at least two years to assess changes resulting from the amendments. Seedling establishment, growth, and survival of plants will be quantified by comparing species presence and productivity within the various treatments in early summer and late fall. The study plots will be monitored for a minimum of five years after the project's implementation.

The eight treatments being evaluated are:

1. Control Plots (no amendments, no water)

2. Control (no amendments) + Water

3. Dairy Manure Compost (2.5 $\mathrm{yd}^{3}$ per plot)

4. Green Waste Compost $\left(2.5 \mathrm{yd}^{3}\right)$

5. Biosolids Compost $\left(2.5 \mathrm{yd}^{3}\right)$

6. Green Waste Compost $\left(5.0 \mathrm{yd}^{3}\right)+$ Water

7. Biosolids Compost $\left(5.0 \mathrm{yd}^{3}\right)+$ Water

8. Cobble Mulch (2-3 inch diameter cobbles spread uniformly on the ground surface as a mulch)

The study's experimental design is categorized as a completely randomized block design containing 24 plots. There are eight treatments with three replicates of each (Figure A-1, Attachment A).

\subsection{Evaluation of Alternative Cover Design Performance}

Two models will be used to predict water balances on the landfill covers and these predicted values will be compared with field data acquired through the ALCD Project. The EPA's Hydrologic Evaluation of Landfill Performance (HELP) model (EPA, 1994) will be used to predict water balances for all six ALCD covers. The HELP model is designed to analyze "resistive" type barriers that may inhibit downward moisture movement under saturated conditions. The three covers installed in Phase I are all "resistive" barriers. The alternative covers installed during Phase $\Pi$ of the project are not "resistive" barriers, but rather are designed to minimize infiltration and percolation under unsaturated conditions. The HELP model is not as accurate in predicting hydrologic processes for the Phase II covers because of its inability to predict water movement under unsaturated conditions. Therefore the TOUGH2 model (Pruess, 1991) will be used to model these three covers. We anticipate that TOUGH2 will yield more accurate predictive results than HELP for the three Phase II alternative landfill covers because of the model's capability for handling water movement under unsaturated conditions (Webb, 1996).

The models used will segregate the water balance variables of each cover into six categories:

1) precipitation $(P)$,

2) surface runoff $(R)$, 
3) lateral drainage $(D)$,

4) evapotranspiration $(E)$,

5) change in soil water storage $(\Delta S)$, and

6) infiltration or percolation (I).

The models predict the percolation through the cover by calculating the following mass balance equation:

$$
I=P-R-D-E-\Delta S \quad \text { (Eq. 3.4-1) }
$$

The field data obtained to verify the computer model predictions will include all of the above water balance variables, with the exception of evapotranspiration which will be calculated by rearranging Eq. 3.4-1 into the following mass balance equation:

$$
E=P-I-R-D-\Delta S \quad \text { (Eq. 3.4-2) }
$$

Instruments to measure runoff and erosion, changes in soil moisture storage, percolation through the cap, and lateral flow were installed within each landfill cover. Meteorological measurements at the ALCD site include precipitation, wind speed and direction, temperature, and solar radiation. Site monitoring began upon completion of the Phase II construction and will continue for a minimum of five years.

Natural precipitation will drive the hydrologic response of the various landfill cover designs. Supplemental water will also be applied through sprinkler irrigation to the eastern half of each cover to hydrologically stress the cover design components.

\subsection{ALCD Tasks}

This test plan elaborates on six project tasks that must be accomplished in order to achieve the goal and objectives of the ALCD. These tasks are:

\subsubsection{Scope}

- Coordinate with Sandia National Laboratories Environmental Restoration Program (SNL-ER), New Mexico Environment Department (NMED), and EPA to scope applicable regulatory requirements for the study through a general review process.

- Assemble a technical review group composed of industry experts, environmental restoration personnel from academia, government organizations, and private industry.

\subsubsection{Site Acquisition, Characterization, and Permitting}

- Select a location for the study.

- Characterize the near-field area, including major fauna and flora association, geology, hydrology, soils, and climatic parameters. 
- Obtain required permits and safety documentation to allow for construction of the demonstration project.

\subsubsection{Design of Landfill Covers and Soil Amendment Plots}

- Phase I - three landfill covers to be designed by ALCD Project Principal Investigator (PD).

- Phase II - establish contracts and construct three additional alternative cover designs, and review and finalize these cover designs. Soil amendment plots to compare the performance of various revegetation technologies will also be established during Phase II.

\subsubsection{Construction of Test Plots}

- Prepare bid documents.

- Establish contracts.

- Acquire and assemble materials and equipment.

- Provide utility rough-ins (water, power).

- Construct test plots and install instrumentation.

\subsubsection{Monitoring of Test Plots}

- Complete installation of all monitoring equipment.

- Prepare data acquisition system.

- Collect data on precipitation, runoff and erosion, infiltration, soil moisture contents, and percolation/leachate from the base of each landfill cover cap as a function of time. Automated data acquisition systems will be employed for most of the measurements.

- Periodically (semi-annually) measure vegetation characteristics (total plant cover, species presence and variation, general production and growth patterns) on each plot.

- Perform additional measurements (e.g., infiltrometer field tests, laboratory tests, etc.) to provide estimates for other parameters needed to calculate water balances and document cover performance.

- Manage data.

\subsubsection{Documentation of Cover Design Performance}

- Document the construction process for each cover.

- Document the costs associated with each cover.

- Summarize, compare, and document water balance data for cover designs to quantify potential benefits of the alternative designs. 
- Compare actual field data with predicted performance from computer modeling (HELP Model and TOUGH2).

- Document recommendations on use of cover alternatives for interim stabilization and/or closure of landfills in arid/semiarid sites.

\subsection{TEST CONFIGURATION}

The six test covers were installed side-by-side to allow for direct comparisons. Each test cover is $13 \mathrm{~m}$ (40 ft) wide by $100 \mathrm{~m}$ ( $300 \mathrm{ft}$ ) long. The $100 \mathrm{~m}$ cover lengths are crowned at mid-section, with half of the length $(50 \mathrm{~m})$ sloping $5 \%$ to the east and the other half sloping $5 \%$ toward the west. Every layer comprising the covers was constructed with a 5\% slope gradients. The covers are level ( $0 \%$ gradient) across their widths. The $100 \mathrm{~m}$ landfill length dimension was chosen because this length is representative of typical hazardous and mixed waste landfills found throughout the DOE complex. The overall landfill covers are approximately two acres in surface area. The western segment of each landfill cover is being monitored under ambient conditions (passive monitoring). The eastern segments of the covers have had a sprinkler system installed for supplemental irrigation to facilitate stress testing (active monitoring).

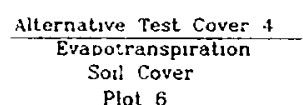

Plot 6
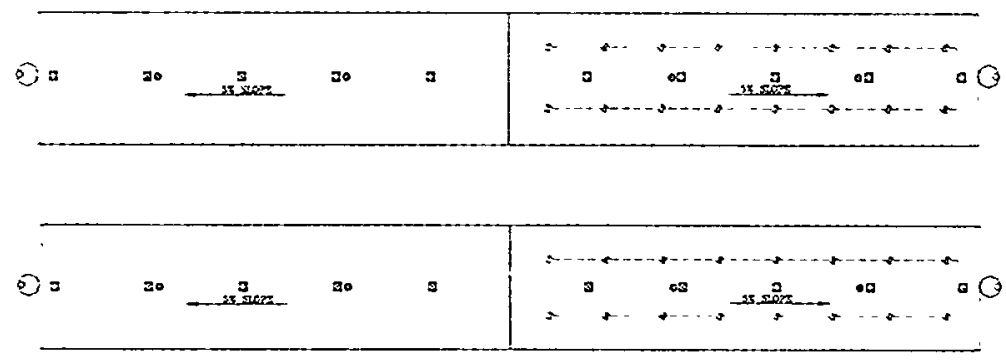

Aiternel:va Test Cover 2
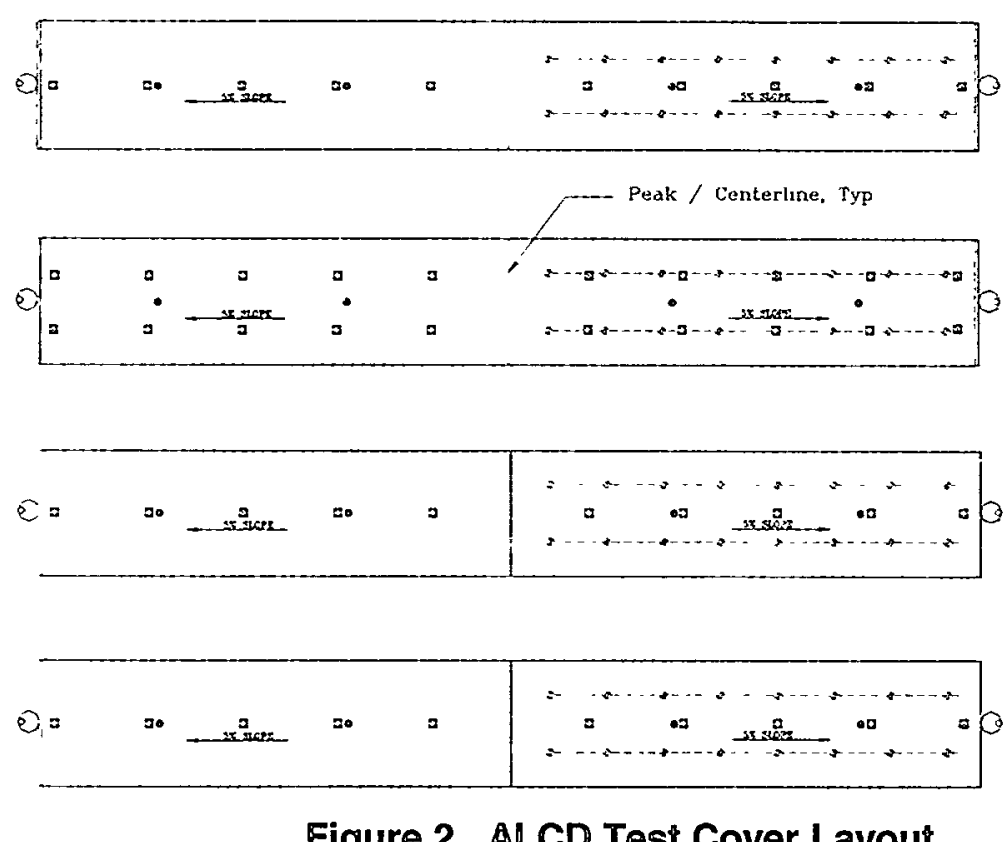

Figure 2. ALCD Test Cover Layout Capllary Barrier Plot *

Baseline Test Cover ? Sust:tle ' $C$ '

Compacted Clay Cover Plot 3

Uternative Test Cover: GCL Cover Plut ?

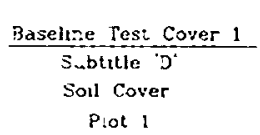

Alternative Test Cover 3 Plot 5

Plot 1 $e$

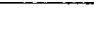

Legend

a TDR Probe, installed by SNi personnel

- Thermocoupie, installed by S.NI. personnel

(3) Nanhole

$\rightarrow-$ Sprinkler System

Direction of Slope 
The covers were designed and constructed using on-site soils. The sections were designed such that the cut volume of earth was approximately equal to the fill required to construct the covers. Therefore, no borrow fill was required. Refer to the drawings in Attachment B (Phase I Covers) and Attachment D (Phase II Covers). Also refer to the specifications in Attachment C (Phase I Covers) and Attachment E (Phase II Covers).

The construction of the test covers closely simulated the construction sequencing that would be seen in the completed fabrication of an actual landfill cover.

\subsection{DESIGN/CONSTRUCTION OF TEST COVERS}

\subsection{Baseline Cover Designs}

\subsubsection{Baseline Test Cover 1 (Plot 1)}

This is a basic soil cover installed to meet minimum requirements for RCRA Subtitle ' $D$ ' regulated landfills (DOE, 1993). Engineered covers for sanitary solid waste landfills must meet these requirements and performance objectives:

1) Cover permeability must be less than or equal to the permeability of the bottom liner/subsoil or no greater than $10^{-5} \mathrm{~cm} / \mathrm{sec}$.

2) Infiltration should be minimized with at least $45 \mathrm{~cm}$ of soil serving as a barrier layer.

3) Erosion should be minimized with at least $15 \mathrm{~cm}$ of topsoil provided for adequate plant establishment and growth.

The completed test cover is $60 \mathrm{~cm}$ thick and is constructed of two layers (Figure 3). The bottom layer is a $45 \mathrm{~cm}$ thick compacted soil barrier layer. The soil barrier layer's primary purpose is to minimize the infiltration and leaching of water into the underlying waste. The top layer consists of $15 \mathrm{~cm}$ of loosely laid topsoil which provides for establishment of vegetation and erosion protection.

The barrier layer was constructed of soil material taken from within the site during excavation. The soil in the barrier layer was placed in a manner to achieve a low hydraulic conductivity using 30,000 to 40,000-pound compaction equipment (moderate weight). Because of the moderate equipment weight, standard proctor was specified as the compaction test method instead of the modified proctor test method, which would require heavier equipment. Whenever a lift was placed above and in contact with a smooth-rolled lift, the top of the smooth-rolled lift was scarified to a depth of one to twoinches to allow the lifts to bond together better; this scarified zone was included in the next loose lift thickness. TDR probes were installed in the test covers between lifts as the covers were constructed. Refer to the drawings in Attachments B and D for TDR locations. 
The topsoil material (originally removed from the site and stockpiled nearby for subsequent use) was placed immediately over the underlying barrier layer without compaction.

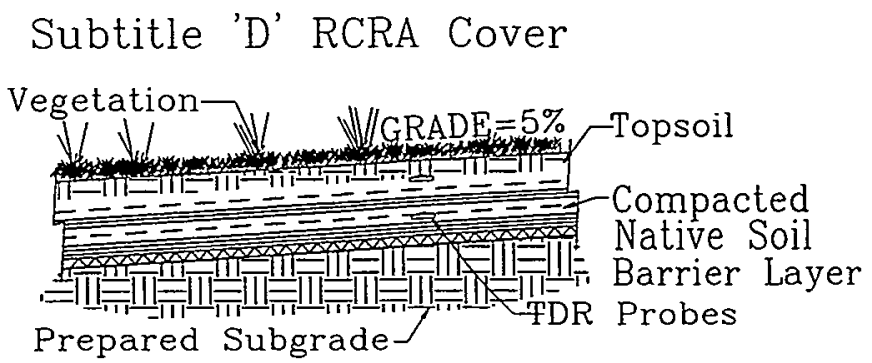

Figure 3. Baseline Test Cover 1

\subsubsection{Baseline Test Cover 2 (Plot 3)}

This test cover contains a Compacted Clay Cover (CCC) designed and constructed according to minimum regulatory requirements for closure of hazardous and mixed waste landfills found in 40 CFR Parts 264 and 265, Subpart N. Under these regulations, owners/operators of landfills are required to perform landfill closures. The primary closure requirements of 264.310 and 265.310 require that the owners/operators design and construct a low-permeability cover over the landfill to minimize migration of liquids into the waste contained therein. Also required are 30 years of post-closure monitoring and maintenance in order to prevent waste migration into the environment. The cover design must provide the following:

1) minimal liquid migration,

2) promote drainage while controlling erosion,

3) minimize maintenance,

4) permeability equal to or less than the permeability of natural subsoil,

5) account for freeze/thaw effects, and

6) accommodate settling and subsidence so that the cover's integrity is maintained.

The recommended EPA RCRA Subtitle 'C' final cover design (EPA 1991) consists of the following layers from the base to surface of the cover:

1) Low hydraulic conductivity geomembrane/soil layer: 60-cm layer of compacted natural or amended soil with a maximum saturated hydraulic conductivity of $1 \mathrm{x}$ $10^{-7} \mathrm{~cm} / \mathrm{sec}$ in contact with a minimum 40 -mil geomembrane.

2) Drainage layer: $\geq 30-\mathrm{cm}$ sand layer having a minimum saturated hydraulic conductivity of $1 \times 10^{-2} \mathrm{~cm} / \mathrm{sec}$, or a layer of geosynthetic material having similar hydraulic conductivity.

3) Topsoil layer: a surface layer with established vegetation (or an armored surface) and $\leq 60 \mathrm{~cm}$ of unconsolidated soil graded at a 3-5\% slope. 
The Baseline Test Cover 2 was designed with the following features:

The cover is $1.5 \mathrm{~m}$ thick and consists of three layers (Figure 4). The bottom layer is a 60 $\mathrm{cm}$ thick compacted soil layer to serve as a barrier layer for the prevention of downward water movement into the underlying waste. Laboratory tests revealed that the native soil selected for this barrier layer did not meet the required hydraulic conductivity. Thus, an amendment of finer material was needed to meet the required saturated hydraulic conductivity (maximum of $1 \times 10^{-7} \mathrm{~cm} / \mathrm{sec}$ ). The laboratory tests included the evaluation of different combinations of native soil and sodium bentonite amendment to attain a saturated hydraulic conductivity of $\cdot 1 \times 10^{-7} \mathrm{~cm} / \mathrm{sec}$. Through laboratory testing using a rigid-wall permeameter (Boyton and Daniels, 1985), we determined that a mixture of sodium bentonite ( $6 \%$ by weight) with the native soil compacted 'wet of optimum' to $98 \%$ of maximum dry density (per ASTM D698) produced the required hydraulic conductivity. The soil was compacted 'wet of optimum' as recommended by the EPA in order to remold the soil to lower the hydraulic conductivity range. All permeameter tests conducted in the 'wet of optimum' range yielded hydraulic conductivity's ranging between $10^{-8}$ to $10^{-9} \mathrm{~cm} / \mathrm{sec}$.

A 40 mil linear low density polyethylene geomembrane was placed directly on the soil barrier layer. The soil barrier layer surface was rolled smooth to ensure intimate contact between the soil and the geomembrane, thereby creating a composite barrier layer.

Eight perforations (approximately one centimeter in diameter) were purposely inserted into the barrier layer geomembrane to simulate what might actually occur under field conditions. The number of perforations was based on an average number of penetrations found in a geomembrane placed in a landfill constructed with average quality control (EPA, 1994).

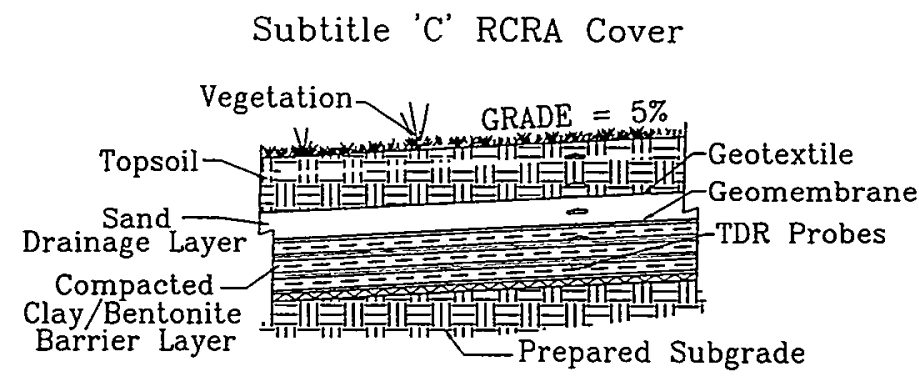

Figure 4. Baseline Test Cover 2

The cover's middle layer is a $30 \mathrm{~cm}$ thick drainage layer designed to minimize the time infiltrated water remains in contact with the underlying barrier layer. The drainage layer does this by quickly re-routing water that has passed through the topsoil layer laterally to collection drains normally located at the perimeter of the landfill. This layer consists of sand placed directly over the geomembrane. The sandy material used was specified to have a minimum saturated hydraulic conductivity of $1 \times 10^{-2} \mathrm{~cm} / \mathrm{sec}$. The sand material was not to contain more than $5 \%$ by weight of fines passing through a No. 200 sieve $(0.074 \mathrm{~mm}$ diameter) to minimize the risk of future clogging of the drainage layer. $\mathrm{A}$ 
non-woven polyester needle-punched geotextile that serves as a filter between the drainage layer material (sand) and overlying topsoil layer was placed directly between the two zones.

The uppermost layer is a $60 \mathrm{~cm}$ thick topsoil layer consisting of uncompacted soil; the layer was constructed with $45 \mathrm{~cm}$ of native soil covered by $15 \mathrm{~cm}$ of topsoil. This layer's primary purpose is to provide a vegetation growth medium and protect the underlying layers from harmful freeze/thaw cycles and surface erosion. The topsoil layer allows for storage of infiltrated water that can later be evaporated.

\subsection{Alternative Cover Designs}

Design criteria for the Alternative Cover designs were very basic. These covers are to be: (1) less expensive than the Baseline Test Cover 2, (2) more effective than the baseline covers at preventing the infiltration of water into the underlying waste in arid environments, and (3) easier and more reliable to construct. The native soil used on the construction of the Alternative Covers came from on-site cut excavations. Other materials purchased off-site, such as the sands and gravels used, were common and readily available materials (i.e., no exotic grain-size distributions, etc.).

Any compaction of soil specified by design for the Alternative Covers was compacted 'dry of optimum' rather than 'wet of optimum' as currently recommended by the EPA with the standard regulatory covers. Dry-side compaction should result in a compacted barrier soil that is less susceptible to potential cracking due to desiccation. Dry-side compaction also allows for easier construction and therefore less expense overall. Furthermore, dry-side compaction should result in greater soil water storage capacity due to the retention of more pore space in the soil matrix.

The decision to compact soil 'dry of optimum' for all the Alternative Covers was supported by laboratory testing that showed the saturated hydraulic conductivity of the soil is relatively insensitive to the molding water content. The hydraulic conductivity was measured in three soil test specimens. One of the specimens was compacted 'wet of optimum', the second was compacted at the 'optimum' water content, and the third was compacted 'dry of optimum'. The standard Proctor curves confirmed the relevant insensitivity of the hydraulic conductivity to the water content.

\subsubsection{Alternative Test Cover 1 (Plot 2)}

This is a Geosynthetic Clay Liner Cover (GCL) identical to the traditional regulatory compacted clay cover with one major difference. The problematic clay barrier layer in the traditional compacted clay cover was replaced with a manufactured geosynthetic clay liner (GCL) (Figure 5). All other design aspects of the cover are similar to those of Baseline Test Cover 2. The overall thickness of the GCL cover is $90 \mathrm{~cm}$ and includes (from bottom to top) a barrier layer (the GCL membrane covered with a geomembrane comprises the composite barrier layer), a sand drainage layer, a geotextile filter fabric, and a topsoil layer, respectively. The geomembrane had the same number of perforations inserted into it as the Baseline Test Cover 2 (Plot 3). The GCL sheet is a composite of 
two non-woven fabrics sandwiching a layer of bentonite. The saturated hydraulic conductivity of the GCL per the manufacturer is $5 \times 10^{-9} \mathrm{~cm} / \mathrm{sec}$ (Claymax, 1995).

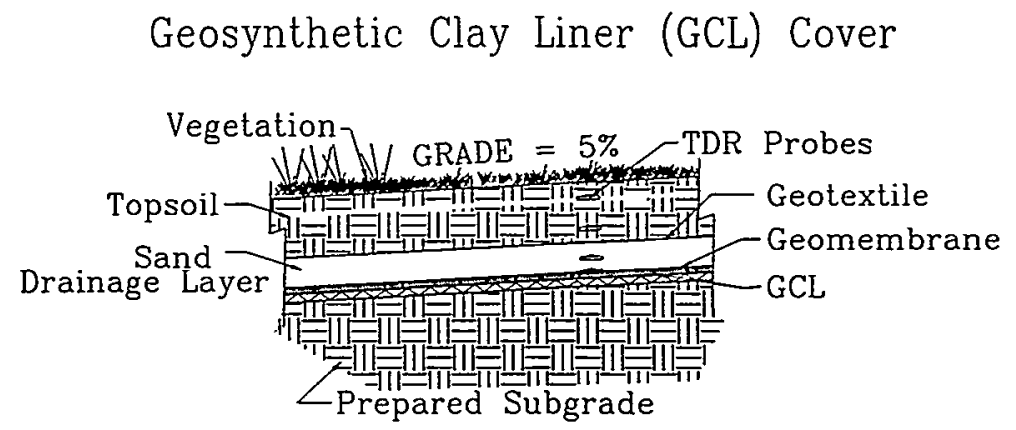

Figure 5. Alternative Test Cover 1

\subsubsection{Alternative Test Cover 2 (Plot 4)}

This cover constitutes a capillary barrier. In the Capillary Barrier Cover (CBC), differences in pore size distribution and thus, differences in matrix potential characteristics between two soil layers will cause water to be retained in the upper soil layer under conditions of unsaturated flow. This phenomenon will occur as long as the contrast in unsaturated properties (e.g., soil-moisture retention and unsaturated hydraulic conductivity) of the soils in the two layers is sufficiently large. In general, the upper soil layer must consist of a soil with a substantially higher matrix potential (i.e., moisture retention) at a similar water content than the lower soil layer for downward water movement to be restricted under unsaturated conditions. In essence, the capillary barrier effect results when a 'relatively fine-grained soil' overlies a "relatively coarse-grained soil". In theory, the capillary pressure head in the fine-grained upper soil layer must approach a value near zero (i.e., saturated conditions) before any appreciable flow occurs into the underlying coarse-grained soil.

Design considerations for the CBC system included determining the proper slope gradient and soil layer thicknesses to minimize the percolation of water through it. In general, recommended layer thicknesses and slope gradients depend upon: (1) climatological information for the site where the cover is to be constructed (e.g., annual precipitation, temperature, humidity, and evaporation), (2) the characteristics of the soils used in the cover (e.g., , moisture retention characteristics, hydraulic conductivity), and other subordinate factors (e.g., slope stability, desiccation considerations). Case studies (Melchoir et al. 1994; Benson and Khire 1995) were used as bases for these design considerations.

The CBC system includes four primary layers: (1) a topsoil layer, (2) an upper drainage layer, (3) a barrier soil layer, and (4) a lower drainage layer (Figure 6). The topsoil layer is $30 \mathrm{~cm}$ thick. In addition to providing a medium for plant growth, this surface layer is placed to enhance evapotranspiration (ET) and minimize desiccation in the barrier soil layer. Vegetation establishment and growth increases ET and protects the cover against surface erosion. The upper lateral drainage layer is $22 \mathrm{~cm}$ of gravel overlain by $8 \mathrm{~cm}$ of 
sand. The sand serves as a graded filter to prevent topsoil from clogging the underlying drainage layer. The gravel allows for lateral drainage of any water that has percolated through the topsoil. The barrier soil layer $(45 \mathrm{~cm}$ of compacted soil) and lower drainage layer ( $30 \mathrm{~cm}$ of sand) comprise the capillary barrier.

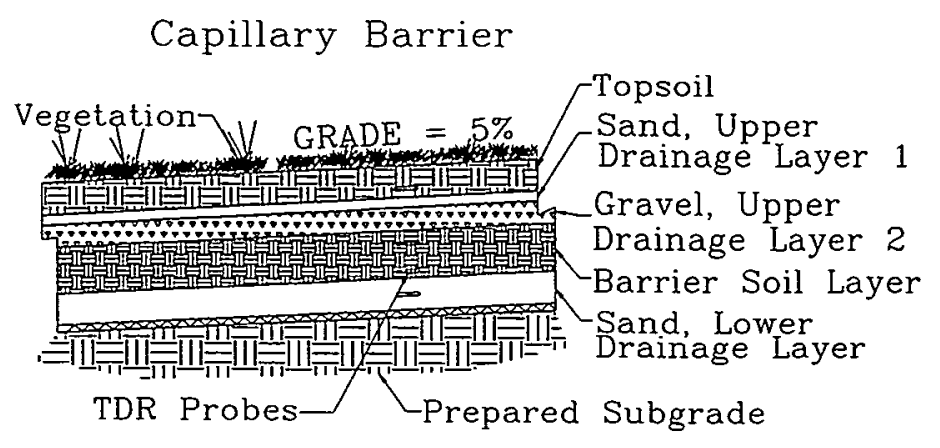

Figure 6. Alternative Test Cover 2

\subsubsection{Alternative Test Cover 3 (Plot 5)}

This cover is referred to as the Anisotropic Barrier (AB) Cover. This system is designed to limit downward water movement while at the same time encouraging lateral movement of water. This cover is composed of a layering of capillary barriers.

The AB Cover (Figure 7) consists of four layers: (1) a gravel-enhanced topsoil layer, (2) a cover soil layer, (3) an interface layer, and (4) a sub-layer. The topsoil layer (15 cm thick) is composed of a mixture of local topsoil and pea-gravel (the gravel to soil mixture is $25 \%$ by weight). The gravel was added to help minimize surface erosion due to surface runoff. The gravel-enhanced topsoil also enhances vegetation growth and evapotranspiration. The underlying cover soil layer is $60 \mathrm{~cm}$ of native soil which functions as plant rooting medium and a storage layer for water that is ultimately subjected to evapotranspiration. The interface layer consists of $15 \mathrm{~cm}$ of fine sand intended as a filter between the overlying soil and the underlying gravel layers. The fine sand interface layer also serves as a drainage layer to laterally divert water that has percolated through the cover soil. The sublayer is composed of $15 \mathrm{~cm}$ of pea-sized gravel. The soil layers over sand layer create one capillary barrier while the sand layer over pea-sized gravel layer creates a second capillary barrier. The interface layer and sublayer combined serve a dual purpose as bio-barriers (e.g., they minimize the penetration or intrusion of roots and burrowing animals into the underlying waste). 
Anisotropic Barrier

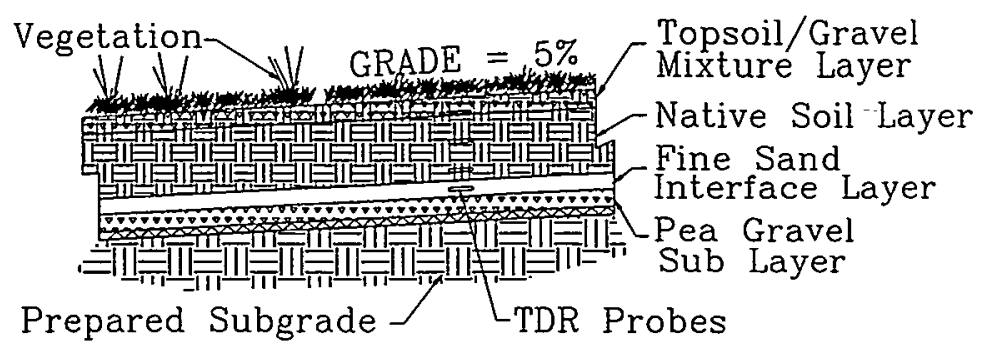

Figure 7. Alternative Test Cover 3

\subsubsection{Alternative Test Cover 4 (Plot 6)}

This cover is referred to as an Evapotranspiration (ET) Cover (Figure 8). The ET Cover consists of a single, vegetated soil layer constructed to represent an optimum mix of soil texture, soil thickness, and vegetation cover. Regional case studies considerations (Nyhan et al. 1986, Nyhan et al. 1990) were used to assist with this cover's design. The studies showed that a well designed, simple soil cover can be very effective in preventing excessive erosion due to runoff and percolation - particularly in dry environments. The vegetated soil cover serves as the principal mechanism inhibiting soil water movement through the cover and into the underlying waste, with the texture and depth of soil providing the means for storing soil moisture in the zone where it is available to plants and most susceptible to evaporation. Many studies have shown that vegetation in arid and semiarid environments has the potential to remove nearly $100 \%$ of the water that infiltrates the soil, as long as plant roots can access the moisture (Hakonson, 1996). Under these circumstances, the potential for deep percolation of soil moisture is minimal. Ideally, the vegetation cover should include an optimum mixture of species, including cool season and warm season species. Additionally, depending upon their rooting habits, species with different growth forms (such as grasses, shrubs, or trees) should be used to revegetate the site in order to distribute the ET over as much of the year as possible. However, caution must be exercised so that deep roots do not penetrate through the landfill cover and breach the landfill's integrity.

The ET Cover is a $90 \mathrm{~cm}$ thick monolithic soil cover. The lower $75 \mathrm{~cm}$ of soil was compacted while the upper $15 \mathrm{~cm}$ was distributed loosely over the site without compaction. The non-compacted soil cover allows for ample water storage and, when combined with a good vegetation cover, will produce high ET rates and greatly reduce the potential for leaching. 


\section{Evapotranspiration Soil Cover}

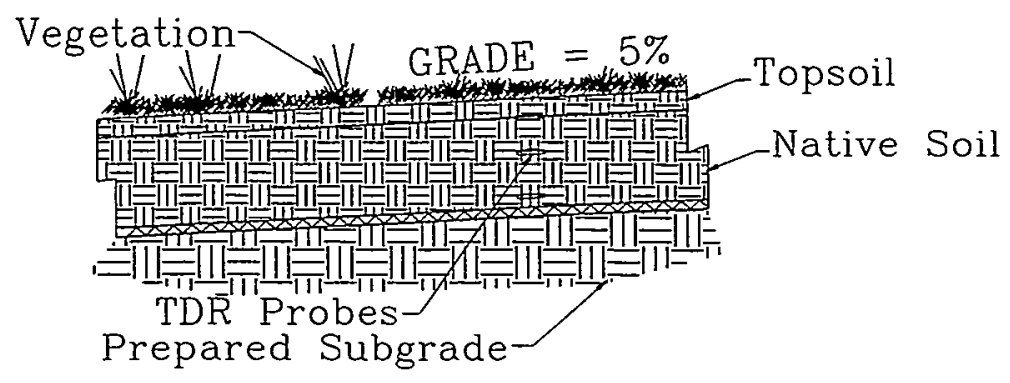

Figure 8. Alternative Cover 4

\subsection{CONSTRUCTION QUALITY ASSURANCE}

\subsection{Personnel Qualifications}

An important factor in assessing the quality of a future constructed product is the degree to which key personnel involved in the process are qualified to perform the required tasks. The key individuals involved in Materials Quality Assurance (MQA)/Construction Quality Assurance (CQA) during the construction of the ALCD Project test covers and their minimum recommended qualifications are listed in Table 6.1.

\subsection{MQAVQA Plan}

A written MQA/CQA plan must precede any field construction activities. Section 6 of this DQMP is an overview of the CQA plan. This plan adheres to the standards recommended in the EPA Technical Guidance Document "Quality Assurance and Quality Control for Waste Containment Facilities" (EPA, 1994).

Most state and federal regulatory agencies require that a MQA/CQA plan be submitted by the owner/operator and be approved by that agency prior to construction of the proposed project. The MQA/CQA plan is usually part of the permit application.

A copy of the site-specific plans and specifications, MQA/CQA plan, and MQA/CQA documentation reports will be retained at the facility by the MQA/CQA engineer. The plans, specifications, and MQA/CQA documents will be the chief means for the facility owner/operator to demonstrate to the regulatory agency that MQA/CQA objectives for a project have been met.

This MQA/CQA plan includes a detailed description of all MQA/CQA activities that were used during materials inspection and construction to manage the installed quality of the ALCD Project landfill covers and associated facilities. The standard EPA MQA/CQA plan has been tailored to the ALCD Project with all important guidelines and standards integrated into the project plans and specifications. 
Table 6.1. Minimum Personnel Qualifications (EPA, 1994)

\begin{tabular}{|c|c|}
\hline Individual & Minimum Qualifications \\
\hline Design Engineer & Registered Professional Engineer. \\
\hline SNL Designated Representative & $\begin{array}{l}\text { The specific individual designated by SNL } \\
\text { with knowledge of the project, and its } \\
\text { plans, specifications, and Quality Control } \\
\text { (QC)/Quality Assurance (QA) documents. }\end{array}$ \\
\hline Manufacturer/Fabricator & $\begin{array}{l}\text { Experience in manufacturing, or } \\
\text { fabricating, at least } 1,000,000 \mathrm{ft}^{2} \text { of similar } \\
\text { geosynthetic materials. }\end{array}$ \\
\hline MQC Personnel & $\begin{array}{l}\text { Manufacturer - or fabricator - trained } \\
\text { personnel in charge of quality control of the } \\
\text { geosynthetic materials to be used in the } \\
\text { specific waste containment facility. }\end{array}$ \\
\hline MQC Officer & $\begin{array}{l}\text { The individual specifically designated by a } \\
\text { manufacturer or fabricator in charge of } \\
\text { geosynthetic material quality control. }\end{array}$ \\
\hline Geosynthetic Installer's Representative & $\begin{array}{l}\text { Experience installing at least } 100,000 \mathrm{ft}^{2} \text { of } \\
\text { similar geosynthetic materials. Must have } \\
\text { an on-site foremen that is NICET trained as } \\
\text { a minimum. }\end{array}$ \\
\hline CQC Personnel & $\begin{array}{l}\text { Employed by the general contractor, } \\
\text { installation contractor, or earthwork } \\
\text { contractor involved in waste containment } \\
\text { facilities; appropriately trained. }\end{array}$ \\
\hline CQA Personnel & Appropriately trained. \\
\hline MQA/CQA Engineer & $\begin{array}{l}\text { Employed separately from the contractor; } \\
\text { Registered Professional Engineer. }\end{array}$ \\
\hline MQA/CQA Certifying Engineer & $\begin{array}{l}\text { Registered Professional Engineer in the } \\
\text { State of New Mexico. }\end{array}$ \\
\hline
\end{tabular}

\subsection{Documentation}

In addition to insuring the correct installation of the ALCD Project landfill covers/facilities, another major intent of this MQA/CQA process is to provide documentation for those individuals who were unable to observe the entire construction process (e.g., representatives 
of the regulatory agency, etc.) so that these individuals can make informed judgments about the quality of construction for the project. MQA/CQA procedures and results were thoroughly documented for the ALCD Project.

\subsubsection{Daily Inspection Reports}

Daily reporting and documentation procedures were required. The MQA/CQA engineer prepared daily written inspection reports that were included in the final MQA/CQA document. The daily reports included information about the work that was accomplished, tests that were performed and observations that were made, and descriptions of the adequacy of the work completed.

\subsubsection{Daily Summary Reports}

A daily written summary was prepared by the MQA/CQA engineer. These reports provide a chronological framework for identifying and recording all other reports and aids in tracking what activities/tasks were completed and by whom. At a minimum, the daily summary reports included the following:

- Date, project name (ALCD), location, waste containment unit under construction, personnel involved in major activities, and other relevant identification information.

- Description of weather conditions, including temperature, cloud cover, and precipitation.

- Summaries of any meetings held and actions recommended or taken.

- Specific work units and locations of construction under way during that particular day.

- Equipment and personnel being utilized in each work task, including subcontractors.

- Identification of areas or units of work being inspected.

- Unique identifying sheet number of geomembranes for cross-referencing and document control.

- Description of off-site materials received, including any quality control data provided by the supplier.

- Calibrations or recalibrations of test equipment, including actions taken as a result of recalibration.

- Decisions made regarding approval (or disapproval) of units of material or of work and/or corrective actions to be taken in instances of substandard or suspect quality.

- Inspection data sheets and/or problem reporting and corrective measures used to substantiate any MQA/CQA decisions described in the previous item.

- Signature of the MQA/CQA engineer. 


\subsubsection{Inspection and Testing Reports}

All observations, results of field tests, and results of laboratory tests performed on- or offsite were recorded on a data sheet. Recorded observations and test results took the form of notes, charts, sketches, or photographs, or a combination of these.

At a minimum, the inspection data sheets include the following information:

- Description or title of the inspection activity.

- Location of the inspection activity or location from which the sample was taken.

- Type of inspection activity and procedure used (reference to standard method when appropriate or specific method described in MQA/CQA plan).

- Unique identifying geomembrane sheet number for cross-referencing and document control.

- Recorded observation or test data.

- Results of the inspection activity (pass/fail); comparison with specification requirements.

- In addition to the individual preparing the data sheet, identification of all personnel involved in the inspection.

- Signature of the MQA/CQA inspector and review signature by the MQA/CQA engineer.

\subsubsection{Problem Identification and Corrective Measures Reports}

A problem is defined as material or workmanship that does not meet the requirements of the plans, specifications, or MQA/CQA plan for a project or any obvious defect in material or workmanship (even if there is conformance with plans, specifications, and the MQA/CQA plan). At a minimum, problem identification and corrective measures reports contain the following information:

- Location of the problem.

- Description of the problem (in sufficient detail and with supporting sketches or photographic information where appropriate) to adequately describe the problem.

- Unique identifying geomembrane sheet number for cross-referencing and document control.

- Probable cause for the problem.

- How and when the problem was identified (reference to inspection data sheet or daily summary report by inspector).

- Where relevant, estimation of how long the problem existed.

- Any disagreement noted by the inspector between him/her-self and contractor about whether or not a problem existed or the cause of the problem.

- Suggested corrective measure(s). 
- Documentation of correction, if corrective action was taken and completed prior to finalization of the problem, and completed corrective measures report (reference to inspection data sheet, where applicable).

- Where applicable, outline of suggested methods to prevent similar problems in the future.

- Signature of the MQA/CQA inspector and review signature of MQA/CQA engineer.

\subsubsection{Drawings of Record}

Drawings of record (better known as "as-built" drawings) were prepared to document the actual lines, grades, and conditions of each component of the ALCD Project. For the landfill cover soil components, the record drawings included survey data that identified lower and upper elevations of a particular component (layer), the plan dimensions of the component, and locations of all destructive test sampling sites. Separate drawings show record cross sections and special features. Refer to Attachments B and D.

\subsubsection{Final Documentation and Certification}

Upon completion of the project, the MQA/CQA engineer prepared a final documentation and certification report. This report included all daily inspection reports, the daily MQA/CQA engineer's summary reports, inspection data sheets, problem identification and corrective measures reports, other documentation (such as quality control data provided by manufacturers or fabricators, laboratory test results, photographs, as-built drawings, internal MQA/CQA memoranda or reports with data interpretation or analyses), and design changes made by the design engineer during construction. The document was certified to be correct by the MQA/CQA certifying engineer.

\subsubsection{Document Control}

The MQA/CQA documents were maintained under a document control procedure. Any modifications to the documents were reported to and agreed upon by all parties involved. An indexing procedure was developed for conveniently replacing pages in the MQA/CQA plan when modifications became necessary; the replacement pages detailed the revision status.

\subsubsection{Storage of Records}

During construction, the MQA/CQA engineer was responsible for all MQA/CQA documents including copies of the design criteria, specifications, plan revisions, and originals of all data sheets and reports. Duplicate records were kept at a separate location (Building 823, Room 4071C.) to prevent the loss of this valuable information if the originals were inadvertently destroyed. 


\subsection{Meetings}

Pre-designated meetings included a pre-bid meeting held prior to bidding of the contract. Also, a pre-construction meeting was held in conjunction with a resolution meeting after the contract had been awarded, but prior to the start of construction activities.

\subsubsection{Pre-Bid Meeting}

The intent of this meeting was used to discuss the MQA/CQA plan and to resolve differences of opinion among the various concerned parties before the project was let for bidding. Holding the pre-bid meeting before formal construction bids were prepared also allowed the companies bidding on the construction to better understand the level of MQA/CQA required on the project. Also, if the bidders identified problems with the MQA/CQA plan, SNL had the opportunity to correct those problems early on in the process.

\subsubsection{Resolution Meeting}

The objectives of the resolution meeting were to establish lines of communication, review construction plans and specifications, emphasize the critical aspects of the project needed to achieve proper quality, begin planning and coordination of tasks, and identify potential factors that might cause difficulties or delays in construction. The meeting was attended by the project's design engineer, representatives of the general contractor and major subcontractors, the MQA/CQA engineer, and the MQA/CQA certifying engineer.

The resolution meeting covered the following activities:

- An individual was assigned to take minutes (a representative of SNL).

- Individuals were introduced to one another and their project responsibilities (or potential responsibilities) were identified.

- Copies of the project plans and specifications were made available for group discussion.

- The MQA/CQA plan was distributed.

- Copies of any special permit restrictions that are relevant to construction or MQA/CQA were distributed.

- The plans and specifications were described, unique design features were discussed (so the contractors would understand the rationale behind the general design), potential construction problems were identified and discussed, and questions from any of the parties concerning the construction were fielded.

- The MQA/CQA plan was reviewed and discussed, with the MQA/CQA engineer and MQA/CQA certifying engineer outlining their expectations and identifying the most critical components of their project participation.

- Procedures for MQC/CQC proposed by installers and contractors were reviewed and discussed.

- Corrective actions to resolve potential construction problems were discussed. 
- Procedures for documentation and distribution of documents were discussed.

- Each organization's responsibility, authority, and lines of communication were discussed.

- Suggested modifications to the MQA/CQA plan that would improve quality management on the project were solicited.

- Climatic variables (e.g., precipitation, wind, temperature) that might affect the construction schedule were discussed.

Familiarizing all project participants with inspection and testing procedures and the criteria for pass/fail decisions (including the resolution of test data outliers) was a key objective of this meeting. Additionally, it was imperative that all parties understand the key problems MQA/CQA personnel had identified and that each party fully understood their roles and responsibilities and the procedures regarding problem resolution.

\subsubsection{Pre-Construction Meeting}

The pre-construction meeting was held in conjunction with the resolution meeting. The meetings were scheduled after the general construction contracts had been awarded and the major subcontractors and material suppliers had been established. The purpose of the pre-construction meeting was to review the details of the MQA/CQA plan, to ensure that the responsibility and authority of each individual was clearly understood, to reach agreement on the established procedures to resolve construction problems, and to establish a foundation of cooperation in quality management. The pre-construction meeting was attended by the design engineer, representatives of the general contractor and major subcontractors, the MQA/CQA engineer, and the MQA/CQA certifying engineer.

The pre-construction meeting included the following activities:

- Assignment of an individual (SNL representative) to take meeting minutes.

- Introduce parties and identify their responsibilities and authority.

- Distribute the MQA/CQA plan, identify any revisions made after the resolution meeting, and answer any questions about the MQA/CQA plan, procedures, or related documentation.

- Discuss lines of project communication.

- Discuss reporting procedures, distribution of documents, the schedule for routine project meetings, and resolution of construction problems.

- Review site requirements and logistics, including safety procedures.

- Review the project design, discuss the most critical construction aspects, and discuss scheduling and sequencing issues.

- Discuss MQC procedures to be employed by the geosynthetics manufacturer(s).

- Discuss CQC procedures to be employed by the installer or contractor, for example, establish and agree on geomembrane repair procedures. 
- Compile a list of action items requiring resolution and assign responsibilities for these items.

\subsubsection{Progress Meetings}

Weekly progress meetings were held at the job site. At times, additional progress meetings were called at the discretion of the CQA engineer. Meeting attendees were those involved in the specific issues being discussed. These meetings were helpful in maintaining lines of communication, resolving problems soon after they developed, identifying action items, and improving overall quality management. The MQA/CQA engineer or his/her designated representative was present at all meetings.

\subsection{Sample Custody}

All samples were identified in the manner described in the MQA/CQA plan. Whenever a sample was taken, a chain of custody record was made for that sample. If the sample was transferred to another individual or laboratory, records of the transfer were established so that chain of custody could be traced. The purpose for the records of sample custody was to assist in tracing the cause of anomalous test results or other testing problems, and to minimize the potential for accidental sample loss.

Excess soil samples were discarded after testing. Geosynthetic materials sampled during destructive testing were subsampled in triplicate, with one sample tested by CQC personnel, the second sample tested by CQA personnel, and the third retained in storage as prescribed in the CQA plan.

\subsection{Weather}

Weather played a critical role in the construction of the ALCD Project landfill covers. Installation of all geosynthetic materials (including geosynthetic clay liners) and natural clay liners were particularly sensitive to weather parameters. The contractor or installer was responsible for complying with the contract plans and specifications (along with the $\mathrm{MQC} / \mathrm{CQC}$ plans for the various components of the system). Included in the specifications (Attachments $\mathrm{C}$ and $\mathrm{E}$ ) were restrictions on weather conditions for certain construction activities. The contractor or installer was responsible for insuring that these weather restrictions were observed during construction.

\subsection{Work Stoppages}

Unexpected work stoppages resulted from a variety of causes. For example, some testing by other SNL groups on adjacent sites required a half mile radius clearance, encompassing the ALCD site. The MQA/CQA engineer was careful during these work stoppages to determine: (1) whether in-place materials were covered and protected from damage (e.g., lifting of a geomembrane by wind or premature hydration of geosynthetic clay liners), (2) whether partially covered materials were adequately protected from the desiccation (e.g., compacted clay liners), and (3) whether manufactured materials were properly stored and properly or adequately protected from the elements (e.g., protection of geotextiles from excess ultraviolet 
exposure). In essence, the cessation of construction during work stoppages did not mean that MQA/CQA inspection and documentation would temporarily cease.

\subsection{MATERIALS QUALITY ASSURANCE}

This section provides an overview of MQA involved in the construction of the ALCD Project landfill covers and related facilities. Also included is an overview of the level of quality control performed to insure that the material installation met the specifications. Refer to Tables 7.1 through 7.8 for QA specifics adhered to during construction activities. The following subsections describe the intent behind most of the QA requirements.

\subsection{Topsoil Layer}

Quality Assurance of this soil layer accomplished two objectives:

1) ensured the layer materials were suitable, and

2) ensured layer materials were properly placed.

The objective of the surface layers was to provide a medium that allowed for plant establishment and growth. Plant cover reduces the harmful effects of surface erosion resulting from both runoff and wind shear. Each surface layer should be composed of topsoil with adequate levels of plant-essential nutrients to encourage the establishment and productivity non-woody indigenous plants (primarily grasses and forbs). A medium-textured soil, such as a loam with adequate organic matter, will generally meet these requirements.

The surface topsoil layers must provide adequate rooting depth and must not contain too many coarse fragments (gravels, stones, or cobbles) that would limit soil water storage capacity. Additionally, the upper six inches of the soil layer should not be too compacted, as this will also limit plant establishment and growth.

\subsection{Barrier Layer}

Quality Assurance of the compacted soil barrier layers accomplished three objectives:

1) ensured layer material quality met specifications,

2) ensured layer materials were properly placed, and

3) ensured completed layer was properly protected.

The objective of a barrier layer is to provide a uniform layer that has restrictive hydraulic conductivity. To achieve this objective, several materials and construction specifications must be met. The plasticity index (PI) of the soil must be within an acceptable range, the soil must contain a minimum percentage of fines (passing No. 200 sieve $-0.074 \mathrm{~mm}$ diameter) with a maximum percentage of gravel (passing a No. 4 sieve $-4.76 \mathrm{~mm}$ diameter), and no clods or stones larger than the maximum size allowed can be present. 


\subsection{Plasticity Index (PI)}

The soil's PI is the numerical difference between its liquid and plastic limit indices. The liquid limit index is defined as the soil moisture content corresponding to the arbitrary limit between the liquid and plastic states of soil consistency. The plastic limit index is defined as the water content corresponding to the arbitrary limit between the plastic and solid states of consistency of a soil. The higher the soil's PI, the more readily it is compacted and remolded, thus eliminating as many voids (pore space) as possible and lowering the hydraulic conductivity. Caution must be exercised so as not to attain too high a PI because at very high PIs the soil tends to get sticky and clod, and generally become difficult to work with, or lose shear strength.

\subsection{Percent Fines Passing No. 200 Sieve}

A minimum amount of fines are required in fill soils to produce required hydraulic conductivity attributes. Soils with an inadequate quantity of fines (silt and clay-sized particles) typically do not produce a suitably low hydraulic conductivity. Under these circumstances, amendment of fines from external sources may be required. Bentonite clay may be used for this purpose to help fill voids between larger soil particles. Conversely, the amount of fines in the sand and gravel layers were kept to a minimum to reduce the potential for clogging of drainage and retain a higher hydraulic conductivity.

\subsection{Percent Passing No. 4 Sieve}

A maximum percentage of gravel will be maintained in fill soils. Gravel uniformly mixed and totally surrounded by clay does not usually pose a problem in barrier layers. However, problems can occur when the mixture is not uniform and there is a high percentage of gravel in pockets, thereby yielding higher hydraulic conductivity in these areas. Also, gravel in direct contact with the geomembrane can cause damage.

\subsection{Maximum Size of Stones or Debris}

The maximum size allowed for clods and rocks was also specified in the construction plans. Coarse fragments pose a greater potential for problems in dry hard soils than in wet soils. Rocks and other large foreign matter must be removed and clods should be broken down and/or remolded. If a large quantity of these large objects remain in the soil material, higher hydraulic conductivity may occur in portions of the cover.

\subsection{Moisture Content}

Moisture content is the key factor for compaction of a given soil material for several reasons. A high degree of compaction is recommended to attain the lowest possible hydraulic conductivity. The soil must be within a moisture range that enables compaction of the layer to a required high density. An optimum moisture content allows for the maximum dry density to be achieved during compaction. The EPA recommends soil barrier layers be compacted 'wet of optimum' in an effort to maximize the compaction and thus reduce the soil layer's saturated hydraulic conductivity. The higher water content is needed to allow the soil to be remolded to minimize voids when being compacted. Additionally, existing clods in 
the soil material can easily be remolded when wet. An optimum moisture content is also required to prevent the soil's PI from getting so high that the soil becomes sticky and difficult to work with. The shear strength of the soil may also get too low if the soil is wet too much. Another potential major problem may arise when the soil is compacted 'wet of optimum'; the soil may swell and, as it dries, shrinks and cracks known as desiccation cracking.

Compaction of soil barriers in the ALCD alternative covers (Plots 4, 5, and 6) was carried out at 'dry of optimum' moisture contents, yet still within the acceptable moisture range to achieve the required minimum dry density. The reason for this is that the design for the alternative covers is based on effective waste isolation under conditions of unsaturated soils. The more important criteria for these soil layers is that desiccation cracking be kept to a minimum. Added benefits of 'dry of optimum' compaction are that the completed soil layers will have greater water storage capacity than if they were compacted under wetter soil conditions and drier soils can be easier to work with during construction.

\subsection{Compaction}

The soil layers in the various covers at the ALCD site had different compaction requirements. In general, smooth-rolled vibratory compaction was used \{Plots 1, 3 (top lift), 4, and 6\}. In those cases where kneading compaction was employed (Plot 3), the depth of the compactor feet was substantially less than the loose lift thickness so as not to damage instrumentation placed in the prior lift. With kneading, the soil can more readily be remolded and compressed, as compared to smooth-rolled compaction. The energy (compaction equipment weight) that goes into the compaction, as well as the number of passes, is also important. Standard proctor was specified for this project because the heavier equipment required to achieve modified proctor was not practical. Obviously, the heavier the equipment and the more passes, the higher the compaction. Loose lift thicknesses were specified primarily based on past experience.

\subsection{Bond Between Lifts}

It is crucial that each lift be bonded to the previous lift. This cuts down on the creation of a inter-lift passageways (cracks) for the water to travel along as it passes from an overlying lift to a lower one. To minimize the creation of inter-lift passageways, each lift was scarified to a depth of 1-2 inches prior to the placement of the next lift thus establishing continuity between the lifts.

\subsection{In-Situ Hydraulic Conductivity (Infiltration)}

Hydraulic conductivity will hereafter be interchangeably used with the term "permeability". Permeability is defined as the ease with which gases, liquids, or plants roots penetrate or pass through a bulk mass of soil (or any porous medium) (Fairbridge and Finkl, 1979). In this report, the term permeability primarily refers to the rate of water flow or percolation through

a soil layer. Presumably, the lower the hydraulic conductivity of a given soil cover layer, the better the cover prevents the percolation of water through it into the underlying waste. Two test pads were constructed adjacent to the test cover plots to evaluate in-situ saturated hydraulic conductivity for the various soil materials used in the barrier layers of the two 
Baseline Test Covers. The two test pads have plan dimensions of 7.3-m by 7.3-m and were constructed similarly to the barrier layers in Plot 1 (Subtitle D RCRA Cover) and Plot 3 (Subtitle C RCRA Cover), respectively. A double-ring infiltrometer with a sealed-inner ring was installed on each test pad and the tests were carried out following the guidelines outlined in ASTM D5093.

\subsection{Geomembrane}

The objectives of a geomembrane is to prevent the downward percolation of liquids and to withstand the weight of construction traffic as the test covers are built. The geomembrane used was a 40 mil linear low density polyethylene (LLDPE) liner. Attachment C, Section 02776 describes in detail the QA requirements that ensure an effective membrane was installed. In general, the QA guidelines require that the membrane be installed with no defects. All seams were tested for continuity and adequate strength.

\subsection{Geonet}

The geonet installed below all of the test covers is intended to allow for rapid and free flowing lateral drainage of percolation and also withstand construction traffic during the installation of the test covers. Attachment $\mathrm{C}$, Section 02779 describes in detail the quality assurance required to ensure an effective drainage layer is installed. In general, the $\mathrm{QA}$ requirements specify that the geonet be continuos and undamaged across the entire reach of the cover. Additionally, all seams were required to be adequately connected with plastic ties.

\subsection{Geotextile}

The geotextile installed in the test covers is intended to act as filter fabric to prevent overlying soil from mixing with the underlying layer, yet allowing for free flowing drainage through it. The geotextile should also withstand construction traffic during the installation of the test covers. Attachment C, Section 02778 describes in detail the QA requirements to ensure effective geotextile installation. In general, the requirements specify that the geotextile be continuos and undamaged across the entire reach of the cover and that all seams were to be adequately overlapped.

\subsection{Geosynthetic Clay Liner (GCL)}

The objectives of the geosynthetic clay liner (GCL) installed in Alternative Test Cover 3 (Plot 2) was to prevent the downward percolation of liquids. The GCL used, a product manufactured by Claymax, was required to maintain its integrity when subjected to the compaction caused by traffic during the project contraction. The liner is composed of two nonwoven fabric sandwiching a thin layer of bentonite. Attachment C, Section 02777 describes in detail the QA required to ensure an effective membrane is installed. In general, the QA requirements specify that the GCL be continuous and undamaged across the entire reach of the cover and that all seams were to be adequately overlapped. 


\subsection{Sand/Gravel Layer}

Quality assurance requirements for materials used as sand/gravel layers were intended to meet two objectives:

1) ensured the layer materials were suitable, and

2) ensured layer materials were properly placed.

Each cover design posed different concerns for their respective specified sand/gravel layer(s). The key concerns were that a specified hydraulic conductivity was met, a maximum percent of fines was not exceeded, and that the material fell within an acceptable particle-size distribution (PSD). An appropriate PSD helped ensure material uniformity and that hydraulic conductivity and filtration requirements were met. Fulfillment of these requirements was important to ensure that two distinct adjoining soil layers did not intermix in those covers where a geotextile liner or other synthetic material was not placed between them.

TABLE 7.1. Tests/Observations for Test Covers Subgrade

\begin{tabular}{llll}
\hline Parameter & Method & Minimum Frequency & Limiting Criteria \\
\hline Compaction & ASTM D698 & 1 & $95 \% \mathrm{~min}$. \\
Compaction & ASTM D1556 & $1 /$ cover & $95 \% \mathrm{~min}$. \\
$\begin{array}{l}\text { Compaction } \\
\begin{array}{l}\text { Max. size stone/debris } \\
\text { on surface }\end{array}\end{array}$ & ASTM D2922 & $5 /$ cover & $95 \% \mathrm{~min}$. \\
\hline
\end{tabular}


TABLE 7.2. Tests/Observations for Material in Soil Cover (Plot 1)

\begin{tabular}{|c|c|c|c|}
\hline Parameter & Method & Min. Frequency & Limiting Criteria \\
\hline $\begin{array}{l}\% \text { of fines (No. } 200 \\
\text { sieve) }\end{array}$ & ASTM D422 or D1140 & 2/lift/cover & $20 \%$ minimum \\
\hline $\begin{array}{l}\% \text { of gravel (No. } 4 \\
\text { sieve) }\end{array}$ & ASTM D422 & 2/lift/cover & $10 \%$ maximum \\
\hline Max. size clod/stone & observation & continuous & 5-cm dia. max. \\
\hline Bentonite content & observation & continuous & $\begin{array}{l}6 \% \text { minimum by } \\
\text { weight }\end{array}$ \\
\hline Water Content & ASTM D2216 & 1/lift cover & wet of optimum (1) \\
\hline Water content & ASTM D3017 & 5/lift/cover ${ }^{(4)}$ & wet of optimum ${ }^{(1)}$ \\
\hline Compaction & ASTM D698 & 1 & $95 \%$ minimum \\
\hline Compaction & ASTM D1556 & 1/lift/cover & $95 \% \min$. \\
\hline Compaction & ASTM D2922 & 5/lift/cover ${ }^{(4)}$ & $95 \% \min$. \\
\hline Compactor weight & observation & record actual wgt. & 30,000 lbs. $\min .^{(2)}$ \\
\hline Compaction passes & observation & (3) & (3) \\
\hline Lift thickness & observation & (3) & $9 "$ maximum (loose) \\
\hline $\mathrm{PI}$ & ASTM D4318 & 1/lift/cover & 0 to $35 \%$ \\
\hline $\begin{array}{l}\text { In-Situ Hydraulic } \\
\text { Conductivity }\end{array}$ & ASTM D5093 & $1 /$ cover & $1 \times 10^{-5} \mathrm{~cm} / \mathrm{sec}$ \\
\hline \multicolumn{4}{|c|}{$\begin{array}{l}\text { (1) Must be within acceptable range within standard proctor curve } \\
\text { (2) Recommended min. wgt. To coincide with use of standard proctor curve } \\
\text { (3) To be determined in the field } \\
\text { (4) If an outlier is found } 2 \text { more tests must be taken }\end{array}$} \\
\hline
\end{tabular}


TABLE 7.3. Tests/Observations for Material in GCL Cover (Plot 2)

\begin{tabular}{|c|c|c|c|c|}
\hline Parameter & Method & Min. Frequency & $\begin{array}{l}\text { Limiting Criteria } \\
\text { Vegetation Layer }\end{array}$ & $\begin{array}{l}\text { Limiting criteria } \\
\text { Sand Drainage Layer }\end{array}$ \\
\hline $\begin{array}{l}\% \text { of fines } \\
\text { (No. } 200 \text { sieve) }\end{array}$ & $\begin{array}{l}\text { ASTM D422 } \\
\text { or D1140 }\end{array}$ & 2/lift/cover & $20 \%$ minimum & $5 \%$ maximum \\
\hline $\begin{array}{l}\% \text { of gravel } \\
\text { (No. } 4 \text { sieve) }\end{array}$ & ASTM D422 & 2/lift/cover & $10 \%$ maximum & \\
\hline Compaction & observation & continuous & loose, uniform & loose \\
\hline $\begin{array}{l}\text { Max. size } \\
\text { clod/stone }\end{array}$ & observation & continuous & 5-cm max. & \\
\hline $\begin{array}{l}\text { In-Situ Hydraulic } \\
\text { Conductivity }\end{array}$ & ASTM D5093 & $\begin{array}{l}\text { 1/cover - supplier's } \\
\text { submittal }\end{array}$ & & $1 \times 10^{-2} \mathrm{~cm} / \mathrm{sec}(\mathrm{min})$. \\
\hline $\begin{array}{l}\text { (1) Must be within } \\
\text { (2) Recommended } \\
\text { (3) To be determin } \\
\text { (4) If an outlier is } \mathrm{f}\end{array}$ & $\begin{array}{l}\text { eptable range wi } \\
\text { n. wgt. To coinci } \\
\text { in the field } \\
\text { nd, } 2 \text { more tests } r\end{array}$ & $\begin{array}{l}\text { in standard proctor cur } \\
\text { with use of standard p } \\
\text { ast be taken }\end{array}$ & ctor curve & \\
\hline
\end{tabular}




\section{TABLE 7.4. Tests/Observations for Material in Subtitle ' $C$ ' - Compacted Clay Cover (Plot 2)}

\begin{tabular}{|c|c|c|c|c|c|}
\hline Parameter & Method & Min. Frequency & $\begin{array}{l}\text { Limiting } \\
\text { Criteria } \\
\text { Vegetation } \\
\text { Layer }\end{array}$ & $\begin{array}{l}\text { Limiting Criteria } \\
\text { Sand Drainage } \\
\text { Layer }\end{array}$ & $\begin{array}{l}\text { Limiting Criteria } \\
\text { Soil Barrier Layer }\end{array}$ \\
\hline $\begin{array}{l}\% \text { fines (No. } 200 \\
\text { sieve) }\end{array}$ & $\begin{array}{l}\text { ASTM D422 or } \\
\text { D1140 }\end{array}$ & 2/lift/cover ${ }^{(4)}$ & $20 \% \min$. & $5 \%$ maximum & $20 \%$ minimum \\
\hline $\begin{array}{l}\% \text { gravel (No. } 4 \\
\text { sieve) }\end{array}$ & ASTM D422 & 2/lift/cover & $10 \% \max$. & & $10 \% \max$. \\
\hline Max. size clod/stone & observation & continuous & 2" dia. max. & & 2" dia. max. \\
\hline Bentonite content & $\begin{array}{l}\text { observation - SNL } \\
\text { present during } \\
\text { mixing }\end{array}$ & (3) & & & $6 \%$ by weight, $\min$. \\
\hline Water Content & ASTM D2216 & 1/lift cover & & & wet of optimum \\
\hline Water content & ASTM D3017 & $5 /$ ifft/cover ${ }^{(4)}$ & & & wet of optimum \\
\hline Compaction & ASTM D698 & 1 & loose & loose & $98 \% \min$. \\
\hline Compaction & ASTM D1556 & 1/lift/cover & loose & loose & $98 \% \min$. \\
\hline Compaction & ASTM D2922 & 5/lift/cover ${ }^{(4)}$ & loose & loose & $98 \% \min$. \\
\hline Compactor weight & observation & $\begin{array}{l}\text { record actual } \\
\text { wgt. }\end{array}$ & & & $30,000 \mathrm{lbs}^{(5)}$ \\
\hline Compaction passes & observation & (3) & (3) & & \\
\hline Lift thickness & observation & (3) & & & 23-cm max. (loose) \\
\hline PI & ASTM D4318 & $1 /$ lift/cover ${ }^{(4)}$ & & & 10 to $35 \%$ \\
\hline $\begin{array}{l}\text { Hydraulic } \\
\text { Conductivity }\end{array}$ & ASTM D5093 & $\begin{array}{l}1 \text { test plot per } \\
\text { cover, plots } \\
1 \& 3 \\
\end{array}$ & & $.01 \mathrm{~cm} / \mathrm{sec}, \min$ & $\begin{array}{l}1 \times 10^{-7} \mathrm{~cm} / \mathrm{sec} \\
\max .\end{array}$ \\
\hline $\begin{array}{l}\text { (1) Must be calibrated } \\
\text { (2) Must be calibrated } \\
\text { (3) To be determined } \\
\text { (4) If an outlier is four } \\
\text { (5) Recommended wei }\end{array}$ & $\begin{array}{l}\text { using microwave o } \\
\text { using sand cone } \\
\text { in the field } \\
\text { nd, } 2 \text { more tests mus } \\
\text { ight }\end{array}$ & en technique & & & \\
\hline
\end{tabular}




\section{TABLE 7.5. Tests/Observations for Material in the Capillary Barrier (Plot 4)}

\begin{tabular}{|c|c|c|c|c|c|c|}
\hline Parameter & Method & $\begin{array}{l}\text { Minimum } \\
\text { Frequency }\end{array}$ & $\begin{array}{l}\text { Limiting } \\
\text { Criteria } \\
\text { Vegetation } \\
\text { Layer }\end{array}$ & $\begin{array}{l}\text { Limiting } \\
\text { Criteria } \\
\text { Sand Layer }\end{array}$ & $\begin{array}{l}\text { Limiting } \\
\text { Criteria } \\
\text { Gravel } \\
\text { Layer }\end{array}$ & $\begin{array}{l}\text { Limiting } \\
\text { Criteria } \\
\text { Barrier Soil }\end{array}$ \\
\hline $\begin{array}{l}\text { Max. Size } \\
\text { stone/clod }\end{array}$ & Observation & Continuous & 5-cm dia. & (1) & (2) & 2-cm dia. \\
\hline Compaction & $\begin{array}{l}\text { ASTM } \\
\text { D1556 }\end{array}$ & 1/lift/cover & loose & loose & loose & $95-110 \%$ \\
\hline Compaction & $\begin{array}{l}\text { ASTM } \\
\text { D2922 }\end{array}$ & 5/lift/cover & loose & loose & loose & $95-110 \%$ \\
\hline PI & $\begin{array}{l}\text { ASTM } \\
\text { D4318 }\end{array}$ & $1 /$ cover & - & - & - & $10-35$ \\
\hline $\begin{array}{l}\text { Particle Size } \\
\text { Distribution }\end{array}$ & $\begin{array}{l}\text { ASTM } \\
\text { D422 }\end{array}$ & $1 /$ cover & - & (1) & (2) & - \\
\hline$\%$ fines & $\begin{array}{l}\text { ASTM } \\
\text { D1140 }\end{array}$ & $1 /$ cover & - & $5 \% \max$. & $5 \% \max$. & $20 \% \mathrm{~min}$. \\
\hline $\begin{array}{l}\text { Moisture } \\
\text { Content }\end{array}$ & $\begin{array}{l}\text { ASTM } \\
\text { D2216 }\end{array}$ & 1/lift/cover & - & - & - & $\begin{array}{l}\text { dry of } \\
\text { optimum }^{(3)}\end{array}$ \\
\hline $\begin{array}{l}\text { Moisture } \\
\text { Content }\end{array}$ & $\begin{array}{l}\text { ASTM } \\
\text { D3017 }\end{array}$ & 5/lift/cover & - & - & - & $\begin{array}{l}\text { dry of } \\
\text { optimum }\end{array}$ \\
\hline $\begin{array}{l}\text { Lift } \\
\text { Thickness }\end{array}$ & Observation & Continuous & - & - & - & $\begin{array}{l}15-\mathrm{cm} \\
\text { compacted }\end{array}$ \\
\hline $\begin{array}{l}\text { Compactor } \\
\text { Wgt. }\end{array}$ & Observation & $\begin{array}{l}\text { Record } \\
\text { Actual Wgt. }\end{array}$ & - & - & - & $\begin{array}{l}30,000 \# \\
\text { min. }\end{array}$ \\
\hline \%gravel & $\begin{array}{l}\text { ASTM } \\
\text { D422 }\end{array}$ & $1 /$ cover & $10 \% \max$. & - & - & $10 \% \max$. \\
\hline $\begin{array}{l}\text { Hydraulic } \\
\text { Conductivity }\end{array}$ & $\begin{array}{l}\text { ASTM } \\
\text { D5084 }\end{array}$ & $1 /$ cover & - & $0.01 \mathrm{~cm} / \mathrm{sec}$ & $0.1 \mathrm{~cm} / \mathrm{sec}$ & - \\
\hline
\end{tabular}

(1) Attachment E, Section 02200, figures 3 \& 4

(2) Attachment E, Section 02200, figures 3 \& 4

(3) Attachment $E$, section 02200, figure 2 
TABLE 7.6. Tests/Observations for Material in Anisotropic Barrier (Plot 5)

\begin{tabular}{|c|c|c|c|c|c|c|}
\hline Parameter & Method & $\begin{array}{l}\text { Minimum } \\
\text { Frequency }\end{array}$ & $\begin{array}{l}\text { Limiting } \\
\text { Criteria } \\
\text { Vegetation } \\
\text { Layer }\end{array}$ & $\begin{array}{l}\text { Limiting } \\
\text { Criteria } \\
\text { Sand } \\
\text { Layer }\end{array}$ & $\begin{array}{l}\text { Limiting } \\
\text { Criteria } \\
\text { Gravel } \\
\text { Layer }\end{array}$ & $\begin{array}{l}\text { Limiting } \\
\text { Criteria } \\
\text { Compacted } \\
\text { Soil Layer }\end{array}$ \\
\hline $\begin{array}{l}\text { Max. Size } \\
\text { stone/clod }\end{array}$ & Observation & Continuous & 5-cm dia. & (1) & (2) & 5-cm dia. \\
\hline Compaction & $\begin{array}{l}\text { ASTM } \\
\text { D1556 }\end{array}$ & 1/lift/cover & loose & loose & loose & $\begin{array}{l}\text { 100pcf to } \\
110 \mathrm{pcf}\end{array}$ \\
\hline Compaction & $\begin{array}{l}\text { ASTM } \\
\text { D2922 }\end{array}$ & 5/lift/cover & loose & loose & loose & $\begin{array}{l}\text { 100pcf to } \\
110 p c f\end{array}$ \\
\hline PI & $\begin{array}{l}\text { ASTM } \\
\text { D4318 }\end{array}$ & $1 /$ cover & - & - & - & $0-35$ \\
\hline $\begin{array}{l}\text { Particle Size } \\
\text { Distribution }\end{array}$ & ASTM D422 & $1 /$ cover & - & (1) & (2) & - \\
\hline$\%$ fines & $\begin{array}{l}\text { ASTM } \\
\text { D1140 }\end{array}$ & 1/cover & - & $5 \% \max$. & $5 \% \max$. & $20 \% \min$. \\
\hline $\begin{array}{l}\text { Moisture } \\
\text { Content }\end{array}$ & $\begin{array}{l}\text { ASTM } \\
\text { D2216 }\end{array}$ & 1/lift/cover & - & - & - & $\begin{array}{l}\text { dry of } \\
\text { optimum }^{(3)}\end{array}$ \\
\hline $\begin{array}{l}\text { Moisture } \\
\text { Content }\end{array}$ & $\begin{array}{l}\text { ASTM } \\
\text { D3017 }\end{array}$ & 5/lift/cover & - & - & - & $\begin{array}{l}\text { dry of } \\
\text { optimum }\end{array}$ \\
\hline $\begin{array}{l}\text { Lift } \\
\text { Thickness }\end{array}$ & Observation & Continuous & - & - & - & $\begin{array}{l}15-\mathrm{cm} \\
\text { compacted }\end{array}$ \\
\hline $\begin{array}{l}\text { Compactor } \\
\text { Wgt. }\end{array}$ & Observation & $\begin{array}{l}\text { Record } \\
\text { Actual Wgt. }\end{array}$ & - & - & - & $\begin{array}{l}30,000 \# \\
\text { min. }\end{array}$ \\
\hline \%gravel & $\begin{array}{l}\text { ASTM } \\
\text { D422 }\end{array}$ & 1/cover & - & - & - & $10 \% \max$. \\
\hline \%gravel & observation & continuous & $\begin{array}{l}25 \% \text { by } \\
\text { weight }\end{array}$ & - & - & - \\
\hline $\begin{array}{l}\text { Hydraulic } \\
\text { Conductivity }\end{array}$ & $\begin{array}{l}\text { ASTM } \\
\text { D5084 }\end{array}$ & $1 /$ cover & - & $\begin{array}{l}0.01 \\
\mathrm{~cm} / \mathrm{sec}\end{array}$ & $0.1 \mathrm{~cm} / \mathrm{sec}$ & - \\
\hline
\end{tabular}

(1) Attachment E, Section 02200, figure 3

(2) Attachment E, Section 02200, figure 3

(3) Attachment E, Section 02200, figure 5 
TABLE 7.7. Tests/Observations for Material in ET Cover (Plot 6)

\begin{tabular}{|c|c|c|c|c|}
\hline Parameter & Method & Min. Frequency & $\begin{array}{l}\text { Limiting Criteria } \\
\text { Topsoil }\end{array}$ & $\begin{array}{l}\text { Limiting } \\
\text { Criteria } \\
\text { Topsoil }\end{array}$ \\
\hline $\begin{array}{l}\% \text { of fines (No. } 200 \\
\text { sieve) }\end{array}$ & $\begin{array}{l}\text { ASTM D422 or } \\
\text { D1140 }\end{array}$ & 2/lift/cover & $20 \%$ minimum & $20 \%$ minimum \\
\hline $\begin{array}{l}\% \text { of gravel (No. } 4 \\
\text { sieve) }\end{array}$ & ASTM D422 & 2/lift/cover & $10 \%$ maximum & $\begin{array}{l}10 \% \\
\text { maximum }\end{array}$ \\
\hline $\begin{array}{l}\text { Max. size } \\
\text { clod/stone }\end{array}$ & observation & continuous & 5-cm dia. max. & 5-cm dia. max. \\
\hline Water Content & ASTM D2216 & 1/lift cover & - & $\begin{array}{l}\text { dry of } \\
\text { optimum (1) }\end{array}$ \\
\hline Water content & ASTM D3017 & 5/lift/cover ${ }^{(4)}$ & 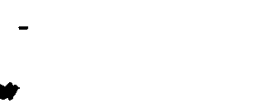 & $\begin{array}{l}\text { dry of } \\
\text { optimum }\end{array}$ \\
\hline Compaction & ASTM D698 & 1 & - & $95 \% \min$. \\
\hline Compaction & ASTM D1556 & 1/lift/cover & - & $95 \% \min$. \\
\hline Compaction & ASTM D2922 & 5/lift/cover ${ }^{(4)}$ & - & $95 \% \min$. \\
\hline Compactor weight & observation & $\begin{array}{l}\text { record actual } \\
\text { wgt. }\end{array}$ & - & $\begin{array}{l}30,000 \mathrm{lbs} . \\
\min ^{(2)}\end{array}$ \\
\hline Lift thickness & observation & (3) & - & $\begin{array}{l}\text { 15-cm max. } \\
\text { (compacted) }\end{array}$ \\
\hline $\mathrm{Pl}$ & ASTM D4318 & 1/lift/cover & - & 0 to $35 \%$ \\
\hline $\begin{array}{l}\text { (1) Must be within acc } \\
\text { (2) Recommended mir } \\
\text { (3) To be determined } \\
\text { (4) If an outlier is four }\end{array}$ & $\begin{array}{l}\text { ptable range withi } \\
\text { wgt. To coincide } \\
\text { the field } \\
2 \text { more tests mus }\end{array}$ & $\begin{array}{l}\text { lard proctor curve } \\
\text { se of standard proc }\end{array}$ & curve & \\
\hline
\end{tabular}

\subsection{Allowable Outliers for Material Tests}

\begin{tabular}{ll}
\hline Parameter & $\begin{array}{l}\text { Maximum Allowable Percentage } \\
\text { of Outliers (if applicable) }\end{array}$ \\
\hline$\%$ fines & $5 \%$ overall, no more than 1 per lift \\
$\%$ gravel & $5 \%$ overall, no more than 1 per lift \\
Max. size clod & $10 \%$ overall, reduce as much as practical \\
Bentonite content & $5 \%$ overall \\
Water content & $5 \%$ overall \\
Compaction & $5 \%$ overall \\
Lift thickness & $5 \%$ overall \\
PI & $5 \%$ overall, no more than 1 per lift \\
\hline
\end{tabular}




\subsection{INSTRUMENTATION/MONITORING EQUIPMENT AND PROCEDURES}

The objectives of the site monitoring activities are to: (1) collect continuous water balance data from each landfill cover design and collect ancillary supporting data from the site for a minimum five-year post-construction period, (2) periodically measure characteristics of the vegetation (total cover and species presence) on each plot, and (3) manage the computer database.

Passive Monitoring: For the first few months after the completion of the test covers, daily onsite observations were required to validate system performance and to correct any problems identified. As the "bugs" in the monitoring system were worked out, on-site observations are necessary less frequently. Continuous data is being obtained for soil moisture status, percolation, inter-flow, runoff, erosion, solar radiation, precipitation, wind speed and direction, relative humidity, air and soil temperatures. Periodic measurements are obtained on the vegetation (percentage ground cover, biomass, leaf area index, and species composition). All of the plots will be monitored for a minimum of five years after construction.

Active Monitoring: Supplemental water will be added through sprinkler irrigation to hydrologically stress the various barriers systems. The sprinkler system was thoroughly tested for rate and uniformity of water application. All monitoring activities employed during the passive monitoring phases of the project will be used here as well.

Data on changes in vegetation cover will be needed to support modeling of evapotranspiration rates and relate these seasonal changes to the annual water balance and the potential for erosion and leaching below the barrier layers of the covers. A photographic record of the changes in vegetation on each cover will also be maintained.

Attributes of the vegetation will be measured seasonally. A point frame will be used to record cover and leaf area indices. Total plant biomass will be determined by clipping and weighing oven-dried samples of the plant material collected from subplots within each cover. Species composition will be measured using line transacts and/or quadrants. These activities commenced when the plot construction was completed.

As previously discussed (Section 3.3), a simplified representation of water balance describes surface runoff and one-dimensional movement of water in the soil profile through the plant rooting depth. For net rates and amounts in centimeters, the water balance equation is:

$$
E=P-I-R-D-\Delta S \quad \text { (Equation 8-1) }
$$

where, precipitation $(P)$, surface runoff $(R)$, lateral drainage $(D)$, evapotranspiration $(E)$, soil water storage $(S)$, and percolation or infiltration $(I)$ are the six water balance variables. All variables in the equation, with the exception of $E$, are being obtained through the site's monitoring systems. Evapotranspiration will be estimated by solving Equation 2. Other variables being measured include temperature, relative humidity, solar radiation, soil temperature by depth, vegetation biomass and cover, and wind speed and direction. Most of these physical 
attributes are being measured with automated monitoring equipment to provide continuous data, while the vegetation characteristics will be recorded manually in the field seasonally.

The instruments that measure the required water balance variables are a combination of commercially available devices and devices developed specifically by engineers associated with the ALCD Project at Sandia National Laboratories (SNL). Most of the commercially-available measuring devices were factory calibrated and could therefore record data immediately upon installation. However, the measuring devices developed specifically for application at the ALCD site \{specifically, the time domain reflectometry (TDR) probes installed in the covers\}, required calibration after they were installed before meaningful data could be obtained.

\subsection{Weather Station}

Weather data required for the ALCD Project is being acquired with an on-site weather station that is a composite of commercially available instruments which measure the different elements of the weather. Wind speed and direction are measured by a 05305 R.M. Young wind monitor. The LI200X Pyranometer measures solar radiation. Temperature and relative humidity measurements are made with an HMP35C temperature/RH probe. A Met One 385 tipping bucket rain gage measures precipitation at the ALCD site. All of the instruments at the site are connected to a Campbell Scientific CR10 data logger which controls how often measurements are made and recorded. In the case of the weather station, measurements are made every ten seconds, however, the measurements are only stored in the CR10's memory once every hour.

\subsection{Instrumentation for Soil Measurements}

Soil water content, humidity, and temperature in the various landfill cover test plots are measured with two types of TDR probes and Type-E thermocouples. The thermocouples measure soil temperature to $60-\mathrm{cm}$ depths in the center of the east and west half-portions of each cover. The thermocouples are calibrated and certified to operate between the temperature range of ten to $32{ }^{\circ} \mathrm{F}$ by the Sandia Standards laboratory. The thermocouples are connected to a Campbell Scientific CR7. The CR7 measures and records soil temperature every hour. Two types of TDR probes measure the soil moisture content throughout the covers. In Phase I (Plots 1, 2, and 3), probes designed and built by Sandia are used to measure the soil moisture content, while in the Phase II covers (Plots 3, 4, and 5) TDR probes developed and manufactured by Campbell Scientific are used. The TDR probes are divided into six stations with Campbell Scientific's Soil Moisture System controlling all of the stations. All stations measure and record values from each probe every two hours. However, both types of probes required rigorous calibration before they could provide accurate and meaningful data.

\subsubsection{Time Domain Reflectometry}

Soil moisture measurements are taken with TDR technology. The TDR process involves sending pulsed waveforms through a coaxial cable to a fixed length probe at some point within a soil and observing the reflected waveform. The reflected waveform is analyzed for two distinct points: the beginning of the probe and the end of the probe. The distance 
between the two points is identified as the apparent length of the probe (Figure 7). The apparent length of the probe is proportional to the square root of the apparent dielectric constant of the soil. Because the dielectric constant of water is relatively high in comparison to other medium (e.g., mineral soil), a signal will propagate slower in a wetter media (i.e., moist soil) than in the same medium when dry. Therefore, probes in soils with a high moisture content have a longer apparent length than probes in drier soils. Further, a mathematical relationship between the volumetric moisture content of the soil and the apparent probe length obtained from the waveform can be established. The resulting equation provides a tool for in-situ volumetric soil moisture content measurements using a time domain reflectometry system.

The main components of Campbell Scientific's TDR Soil Moisture System used in the ALCD Project are a Tektronix 1502B cable tester and a CR10 measurement and control system. The use of the 1502B in conjunction with the CR10 allows for the greatest flexibility in the TDR system. The CR10 contains several different algorithms for wave analysis. For example, the CR10 can measure and store entire waveforms, electrical conductivity, or the ratio of apparent length to actual length of the probe. For the ALCD Project, the CR10 is programmed to output the ratio of the apparent length of the rods to the actual length of the rods $\left(L_{a} / L\right)$; volumetric moisture contents are calculated from the ratio in a separate algorithm.
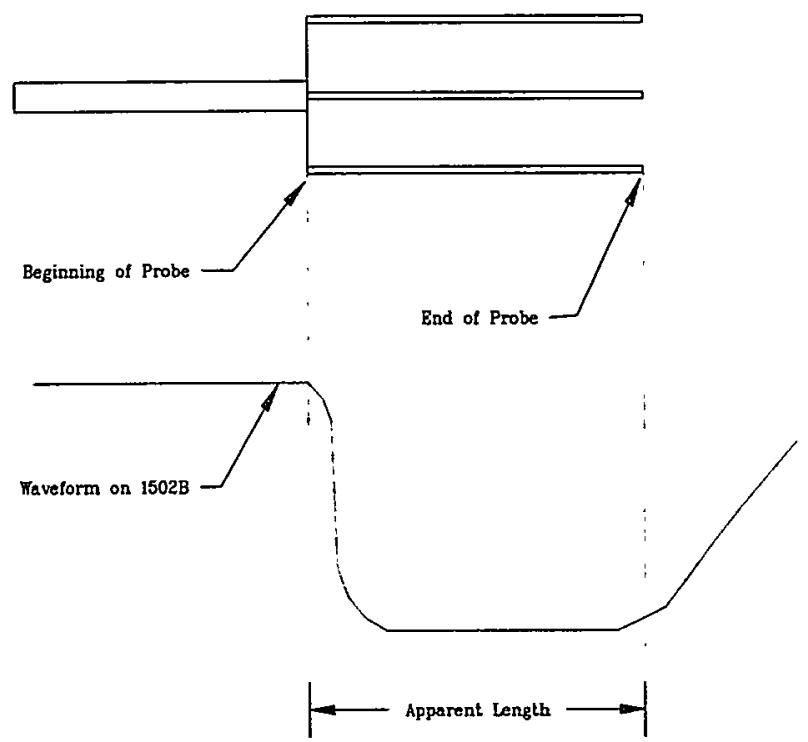

Figure 7. Apparent Length of TDR Probe

The TDR probes used in the Phase I test covers (Plots 1,2, and 3) are the product of extensive research conducted at SNL. During the design of Phase I test covers, commercially available probes reflected a waveform that could not be used because the waveform did not show a rise when the signal reached the end of the probe and the wave end-point could not be determined. This problem necessitated the development of an acceptable probe by SNL personnel for use in the project. Components of the probe, such as rod length, rod spacing, number of rods (two or three), coating the rods (with several 
different types of coatings, coating thickness, and surface preparation), rod diameter, and diode installments were varied until an optimum probe was developed. The resulting probes are composed of a potted PVC housing with a length of RG-8 coaxial cable connected to three equally spaced (six inch exposed length) epoxy coated prongs (Figure 8). The center prong is connected to the signal propagating conductor, while the two outside prongs are connected to the coaxial shield. The epoxy coating inhibits conductivity arising from saline conditions in highly compacted, wet soils.

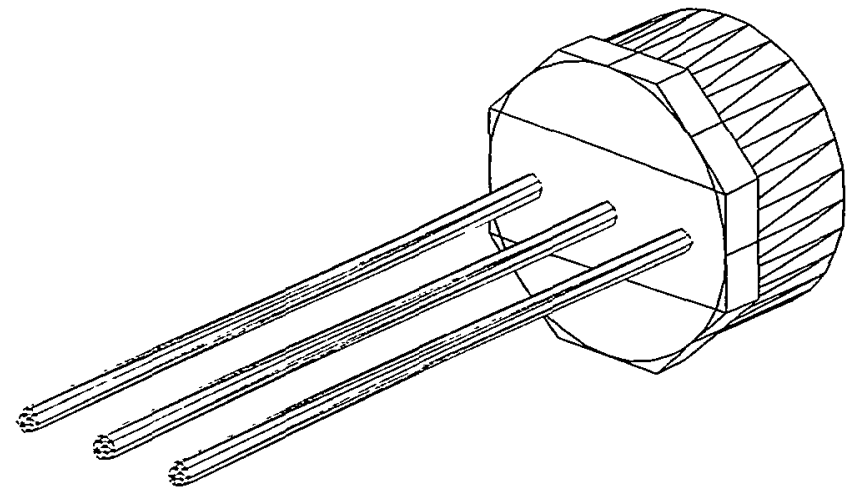

Figure 8. TDR Probe used in Phase I of the ALCD Project

While Phase II was in the design phase, Campbell Scientific released a TDR probe capable of reflecting waveforms under soil conditions similar to the soils used in the Phase II test plots. Thus, the TDR probes for Phase II were purchased from Campbell Scientific rather than expending greater resources designing and building custom probes at SNL (see Figure 9). The Campbell Scientific probes consist of RG-8 coaxial cable terminating in an epoxy body. Within the epoxy body, the center conductor of the cable is connected to the center rod of the three parallel $30-\mathrm{cm}$ long, $1 / 2-\mathrm{cm}$ diameter stainless steel rods. The shield of the coaxial cable is attached to the two outer rods. The rods on the Campbell Scientific probes are not coated due to the increased resolution of the longer rods and the absence of sodium bentonite in the soil materials used in Phase II test covers which allows well defined waveforms to be produced despite the absence of coating on the rods.

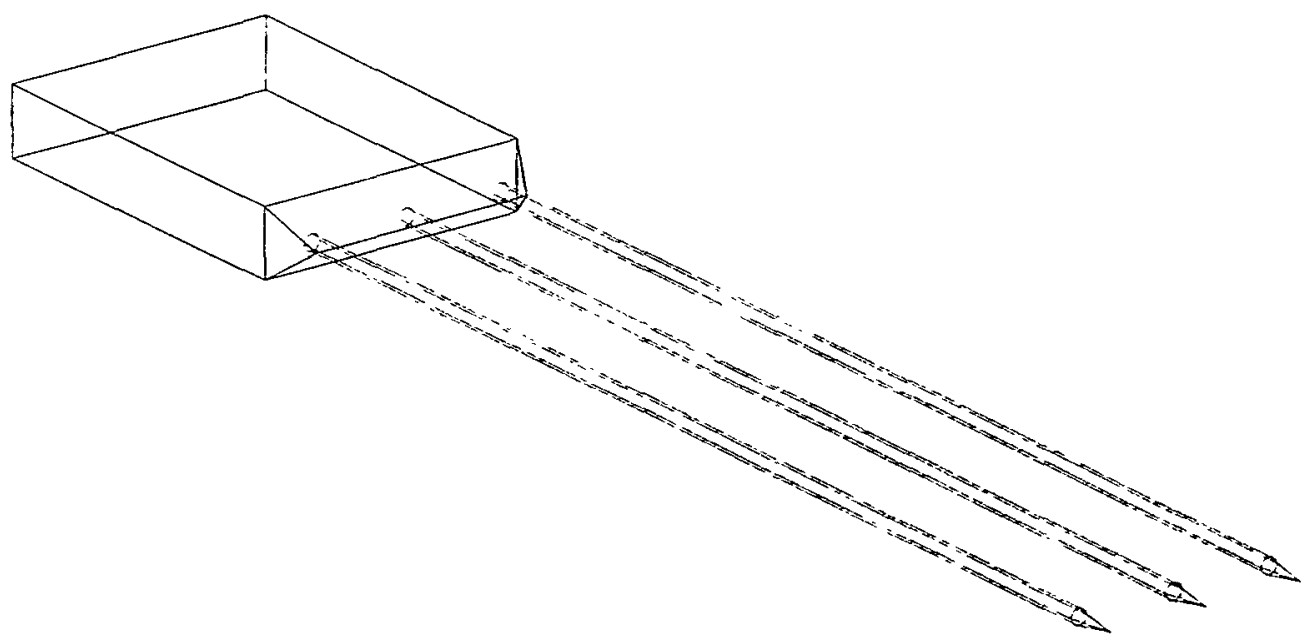

Figure 9. Phase II TDR Probe 


\subsubsection{TDR Calibration for the Phase I Cover Soil Materials}

Many external variables (i.e.; coaxial cable length, soil moisture content, soil type, and soil density) may alter the CR10's analysis of the waveform used to measure the soil moisture content. Therefore, the calibration procedure required the most significant variables likely to be a factor in the soils used in the construction of the ALCD covers be accounted for. During the calibration process the TDR probes in the Phase I covers were placed in four distinct soils: compacted soil mixed with bentonite (six percent by weight), sand, compacted native soil and uncompacted native soil. The CR10 records a different $\mathrm{L}_{\mathrm{a}} / \mathrm{L}$ ratio for similar moisture contents; therefore, calibration data was collected for each of the different soils in Phase I at varying moisture contents. Furthermore, the total cable length of each probe affects the waveform and the resulting $L_{a} / L$ value. After examining the cable lengths that are required for the various soil layers in each plot, five cable lengths which adequately represent the entire array of cable lengths used in the project were identified. Coaxial cables were prepared to allow for the following lengths: $18-\mathrm{m}$, 25-m, 30-m, 37-m, and 42-m. Data was collected for each of these five cable lengths within each soil type during the calibration process.

To achieve proper soil compaction, a customized mold was designed and manufactured by SNL technicians. The mold is similar to a Proctor mold; however, the diameter of the mold is $19.7-\mathrm{cm}$, and the height is $13.3-\mathrm{cm}$. Also, a fitting for a TDR probe was built into the mold. The fitting held the PVC housing of the TDR probes in place while soil was compacted around the probe rods in the mold. The fitting prevented any probe movement and damage to the probe rod coating if the probe was forced into a compacted clod of soil. A thin PVC pipe was cut to fit within the mold and around the probe. The PVC pipe prevents the metal mold from altering the TDR signal. The custom mold allowed for repeatable soil compaction to be achieved throughout the entire calibration procedure.

Calibration for the Six-Percent Bentonite Soil and Compacted Native Soil: Throughout the calibration procedure, representative field conditions were simulated. Samples of each soil material type were taken from their respective stockpiles at the construction site and sieved through a No. 4 sieve ( $4.76 \mathrm{~mm}$ diameter). The resulting soil was then placed in the mold with four equal lifts. The customized mold was approximately twice the size of the standard Proctor mold; therefore, each lift was subjected to 56 compaction blows (more than twice the number required for a standard Proctor test) with a standard Proctor Compaction hammer to simulate the soil density in the test cover's compacted soil layers. The TDR probe was placed in the mold between the second and third lifts. Once compaction was completed, the excess soil was shaved from the top of the mold so that the height of soil in the mold was level with the top of the mold. Ten $\mathrm{L}_{\mathrm{a}} / \mathrm{L}$ values were recorded for each soil material by the CR10. The soil sample was then weighed. After the ten $\mathrm{L}_{\mathrm{a}} / \mathrm{L}$ values were obtained, the sample was broken apart to get a small amount of soil adjacent to the probes to determine its gravimetric water content per ASTM D4643. Water was then added to the same soil sample, and the soil was again mixed to produce a homogeneous sample. This procedure was repeated for five different gravimetric water contents ranging from $1.4 \%$ to $18 \%$. After ten $L_{a} / L$ values were obtained for each of the 
five different water contents, the cable length was changed and the procedure was repeated for each of the five different cable lengths.

Calibration of the Uncompacted Native Soil Material: A similar TDR calibration procedure was repeated for the uncompacted native soil. However, an inconsistent compaction procedure simulated field conditions for this soil material. Samples of soil were again taken from the stockpile in the field and sieved through a No. 4 sieve. The TDR probe was secured into the mold while the soil was placed in the mold and slightly compacted at varying intervals. Once "compaction" was completed, the excess soil was shaved from the top of the mold so that the height of soil in the mold was level with the top of the mold. Ten $L_{a} / L$ values were recorded for each soil material by the CR 10 . The soil sample was then weighed. After the ten $\mathrm{L}_{\mathrm{a}} / \mathrm{L}$ values were obtained, the sample was broken apart to get a small amount of soil adjacent to the probes to determine its gravimetric water content per ASTM D4643. Water was then added to the soil, and the soil is mixed again to produce a homogeneous sample. The procedure was repeated for five different gravimetric water contents ranging from $1.4 \%$ to $18 \%$. When $\mathrm{L}_{\mathrm{a}} / \mathrm{L}$ values were obtained for the five different water contents, the cable length was changed and the procedure was repeated for the five different cable lengths.

Sand: Samples of sand were also taken from the field stockpile of the material. The TDR probe was secured into the mold while the sand was placed in the mold and slightly compacted at varying intervals. Similar to that for the uncompacted native soil, an inconsistent procedure of compaction was used to simulate field conditions. Once compaction was completed, the excess soil was shaved from the top of the mold so that the height of soil in the mold was level with the top of the mold. Ten $\mathrm{L}_{\mathrm{a}} / \mathrm{L}$ values were recorded for each soil material by the CR10. The soil sample was then weighed. After the ten $L_{a} / L$ values were obtained, the sample was broken apart to get a small amount of soil adjacent to the probes to determine its gravimetric water content per ASTM D4643. Water was then added to the soil, and the soil was again mixed to attain homogeneity. The procedure was repeated for five different gravimetric water contents ranging from $1.4 \%$ to $18 \%$. When $\mathrm{L}_{\mathrm{a}} / \mathrm{L}$ values were obtained for the five different water contents, the cable length was changed and the procedure was repeated for the five different cable lengths.

\subsubsection{TDR Calibration Procedure for the Phase Il Cover Soil Materials}

Soil type and cable length were again identified as the most significant variables affecting the CR10's analysis of the waveform for the Phase II cover soil materials. Therefore, data was collected for the three types of soil materials (sand, compacted native soil, and uncompacted native soil) used in constructing the Phase II covers. Calibration data was collected for five representative cable lengths $(26,31,36,42$, and $48-\mathrm{m})$ for the three soil materials.

In order to achieve proper compaction of the soil, a mold was designed and built at SNL to accommodate the larger probes used in the Phase II covers. The mold was constructed from a $0.64-\mathrm{cm}$ steel plate with the following dimensions: $23-\mathrm{cm}$ wide by $61-\mathrm{cm}$. long by 
15-cm deep. This mold size allowed the entire probe to lie flat; consequently, a fitting was not required to hold the probe in place. Soil was compacted around the entire probe within the mold.

Sand: Samples of sand were taken from its respective field stockpile. The TDR probe was secured into the mold while the sand was placed in the mold and compacted slightly at varying intervals. An inconsistent procedure of compaction was used to simulate field conditions. Once compaction was completed, the excess soil was shaved from the top of the mold so the height of soil in the mold was level with the top of the mold. Ten $\mathrm{L}_{2} / \mathrm{L}$ values were recorded for each soil material by the CR10. The soil sample was then weighed. After the ten $L_{a} / L$ values were obtained, the sample was broken apart to get a small amount of soil adjacent to the probes to determine its gravimetric water content per ASTM D4643. Water was then added to the soil, and the soil was again mixed to attain homogeneity. The procedure was repeated for the following approximate gravimetric water contents: $1.5 \%, 5 \%, 7 \%, 11 \%$, and $15 \%$. For the Phase II TDR calibration, the cables used to connect the probes to the $1502 \mathrm{~B}$ were constructed so that the probes would not have to be removed from the soil when the cable lengths were changed. Therefore, the five moisture readings were taken consecutively for each soil material on the same sample.

Compacted Native Soil: Throughout the calibration procedure, field conditions were simulated as much as reasonably possible. Therefore, samples of each soil used in the procedure were taken from their respective stockpiles at the construction site and then sieved through a No. 4 sieve. The resulting soil material was then placed in the mold in three equal lifts. Because the customized mold was a different size and shape than a standard proctor mold, tests were conducted to determine the number of blows required from a Standard Proctor hammer in order to achieve 95\% compaction. The test results showed that 256 blows per lift were required. The TDR probe was placed in the mold in the middle of the second lift. Once compaction was completed, the excess soil was shaved from the top of the mold so that the height of soil in the mold was level with the top of the mold. Ten $L_{a} / L$ values were recorded for each soil material by the CR10. The soil sample was then weighed. After the ten $L_{a} / L$ values were obtained, the sample was broken apart to get a small amount of soil adjacent to the probes to determine its gravimetric water content per ASTM D4643. Water was then added to the soil and the soil was thoroughly mixed again to produce sample homogeneity. The procedure was repeated for the following approximate gravimetric water contents: $1.5 \%, 5 \%, 7 \%, 11 \%$, and $15 \%$. For the Phase II TDR probes, the cables used to connect the probes to the $1502 \mathrm{~B}$ were constructed so that the probe would not have to be removed from the soil when the cable lengths were changed, thereby allowing the consecutive measurement of the five moisture reading for each soil material on the same sample.

Uncompacted Native Soil: A similar TDR calibration procedure was repeated for the uncompacted native soil in Phase II covers as was used in the Phase I calibration. Samples of soil were taken from the field stockpile and passed through a No. 4 sieve. The soil was placed in the mold in three equal lifts. The TDR probe was placed in the 
mold in the middle of the second lift. Each lift received 200 blows with a Standard Proctor Hammer to simulate field compaction conditions. Once "compaction" was completed, the excess soil was shaved from the top of the mold so that the height of soil in the mold was level with the top of the mold. Ten $\mathrm{L}_{\mathfrak{a}} / \mathrm{L}$ values were recorded for each soil material by the CR10. The soil sample was then weighed. After the ten $\mathrm{L}_{\mathrm{a}} / \mathrm{L}$ values were obtained, the sample was broken apart to get a small amount of soil adjacent to the probes to determine its gravimetric water content per ASTM D4643. Water was then added to the soil, and the soil was again mixed to produce a homogeneous sample. The procedure was repeated for the following approximate gravimetric water contents: $1.5 \%$, $5 \%, 7 \%, 11 \%$, and $15 \%$. For the Phase II calibration, the cables used to connect the probes to the $1502 \mathrm{~B}$ were constructed so that the probe would not have to be removed from the soil when the cable lengths are changed; this allowed for the consecutive measurement of the five moisture contents for each soil.

\subsubsection{TDR Equation Derivation}

The first step in developing the TDR equations to accurately measure soil water contents in the field was to convert the wavelength data into volumetric soil moisture contents (see Appendix F). The gravimetric moisture content, total mass of the sample, and volume of the sample were known variables. The volumetric moisture content could therefore be determined from the following equation:

$$
\begin{gathered}
\theta_{v}=\frac{w M_{t}}{(1+w) V_{t}} \\
\text { where: } \theta_{v}=\text { Volumetric Moisture Content } \\
w=\text { Gravimetric Moisture Content } \\
M_{t}=\text { Total Mass of Sample } \\
V_{t}=\text { Total Volume of Sample (Mold) }
\end{gathered}
$$

Once the volumetric moisture content for all of the tests is calculated, the ten $\mathrm{La}_{\mathrm{a}} / \mathrm{L}$ values recorded by the CR10 were averaged for each moisture level. The five volumetric water contents were plotted against the resulting average $\mathrm{L}_{a} / \mathrm{L}$ value for each cable length on an Excel $^{\mathrm{TM}}$ spreadsheet. Using the "Insert Trend Line" feature on Excel, a third order polynomial is found to be the best fit for the data (see Appendix G). After a polynomial equation for each individual cable length was determined, the data from all of the cable lengths in each soil material tested was plotted onto one graph to verify that separate equations were required for the various cable lengths.

Upon examination, the data on the plot containing all of the ratios from the various cable lengths was more scattered than the data on the plots for individual cable lengths. The third order polynomial that fit all of the data did not have as high a correlation as the polynomials that fit the data for individual cable lengths. Therefore, it was apparent that

\footnotetext{
${ }^{n \pi}$ Excel is a registered trademark of Microsoft.
} 
separate polynomials were required for the five different cable lengths for majority of the soil materials tested. The sand, however, only required two equations for the five different cable lengths, one for the shortest cable and another for the remaining four lengths used in Phase I. In Phase I, only one equation was required for sand. The remaining soils require five separate equations for both the Phase I and Phase II samples tested. The equations derived through this process will allow for an adequate approximation of the volumetric water content in all the soil materials used in the ALCD Project through the use of the TDR method.

\subsection{Surface Runoff, Percolation, and Interflow Measurements}

Different methodologies are required to measure surface runoff, percolation/leaching, and lateral drainage. Lateral drainage and percolation are low flow variables. Surface runoff, however, can range from a very high flow rates (fifteen gallons per minute) to a low flow rate (less than one gallon per minute). Therefore, a different approach is required for the measurement of each variable.

\subsubsection{Percolation and Interflow}

Due to the low flow rates, lateral drainage and percolation are measured with tipping buckets. Tipping buckets operate on a simple principle. Water enters the top of a metal cylinder and is funneled to a bucket of known volume within the cylinder. The bucket is separated into two halves and is mounted on a pivot so that water fills each half of the bucket separately. When one half of the bucket becomes full, it tips over and spills its water. The other half of the bucket then moves into position where it can be filled with water, and the entire procedure is repeated each time a half bucket is filled. Each time the bucket tips, a pulse is sent through a signal wire connected to the bucket. The signal wire is connected to the same CR7 data logger that controls the thermocouples; the CR7 counts the number of pulses and multiplies the number of pulses by a calibration factor that indicates the quantity of water emptied.

In the case of the percolation measurements, one pulse is equal to $4.7 \mathrm{ml}$ of water. The volume of water produced by lateral drainage within the plots is expected to be greater than the volume of water produced through percolation. Therefore, a larger tipping bucket is used to measure lateral drainage. For this larger tipping bucket, one pulse equals $13.6 \mathrm{ml}$. The volume measurements for each tipping bucket are determined by the manufacturer and are outlined in the manuals that accompanied the devices. Therefore, the tipping buckets did not require field calibration.

\subsubsection{Surface Runoff}

The complex nature of surface runoff necessitated the use of a more sophisticated system of measurement in order to accurately quantify the amount of water removed from the landfill covers through runoff. During large, high intensity runoff events, a tipping bucket could be flooded before it can tip and therefore result in inaccurate measurement of the quantity of runoff water produced during the event. Additionally, the measuring system had to be capable of operating despite the silt and other debris carried in the flow 
or suspended in the runoff water. A tipping bucket is susceptible to failure with the presence of the silt and other debris in the water. Incorrect volumes will be measured for each tip if sediment is present, and a large piece of debris might even prevent the bucket from tipping entirely. The surface runoff collection and quantification system must be able to operate conditions of very flow rates with silt and other debris present in the water.

The surface runoff quantification system used at the ALCD project was designed to first remove the silt and debris from the runoff water and then measure varying water flow rates (see Figure 10). The system consists of a $46-\mathrm{cm}$ wide by $1.2-\mathrm{m}$ long by $46-\mathrm{cm}$ deep sink, a pump, and an Omega FP-540 flow meter. Each half section of the six plots has a separate runoff measurement system. The sinks operate like small sedimentation tanks, with runoff water entering the sink being forced through a series of baffles within the sink to slow water flow and allow for the settling of silt and debris. The exit drain in the sink is covered with a screen to prevent large, floating particles from entering the pump and causing damage to the pump or the flow meter. A minimum water level must be maintained within the sink in order to achieve the desired effect of a sedimentation tank. When surface runoff enters the sink, a bottom float switch rises and triggers a two hour timer. If the water is below the minimum level, surface runoff that enters the sink will not immediately force the float to rise and accuracy in the runoff measurement is compromised. If the water level in the sink triggers the upper float switch before the two hour timer expires, the pump moves water from the sink through a flow meter and out of the gallery until the sink reaches the minimum water level. If the upper float switch is not triggered within the two hour period, the timer will turn on the pump which, in turn, will then empty the sink to the minimum water level.

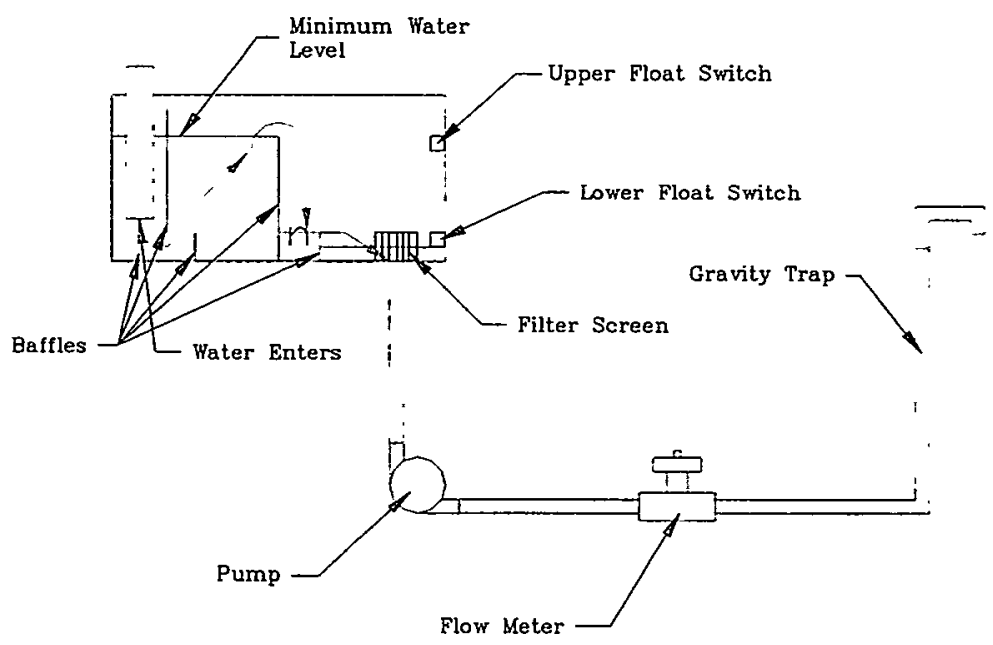

Figure 10. Surface Runoff Water Collection System

The volume of water that passes through the system is quantified with an Omega FP-540 flow meter. The electronic flow meter operates on a similar principle as the tipping bucket. When a fixed amount of water passes through the device, it sends a pulse through 
a signal wire. The signal wire is connected to the CR7 and the CR7 counts the number of pulses and multiplies the number of pulses by a calibration factor that indicates the quantity of water associated with each pulse. Finally, the quantity of water recorded is stored in the CR7's memory. In the event of a very large storm, it is possible for the flow meters to send more pulses than the CR7 can handle. The number of pulses generated by the flow meters and tipping buckets is measured every ten minutes in order to avoid such problems. However, the flow meter requires a steady flow of water to perform properly. Therefore, the pump transmits the water through the flow meter at a steady flow. Furthermore, a gravity trap installed at the end of the PVC water line maintains the pipes full of water thereby preventing air from passing through the flow meter and generating questionable data.

To ensure accurate volumetric measurements, the flow meters were field-calibrated specifically for the ALCD surface runoff collection system. First, a 5-liter vessel was calibrated by the SNL Standards Laboratory. Three volumes of water (5-, 19-, and 38liters) were poured into each respective sink and then pumped through a flow meter. The individual surface water collection system depicted in figure 10 is replicated for each respective slope of each test cover - therefore, there are 12 identical surface water collection systems. The number of pulses for each volume was recorded. The procedure was repeated three times. The data from all of the trials was placed in an Excel spreadsheet. A trend line to fit the data was generated; the resulting equation allows for the determination of the amount of water pumped through each sink to be measured and stored for future calculations (see Appendix $\mathrm{H}$ ).

\subsection{Data Collection and Organization}

The collection and organization of the data is achieved in three stages using a combination of commercially available software from Campbell Scientific Instruments and programs developed from Lab Windows/CVI at Sandia National Laboratories (SNL). In the first stage, data loggers from Campbell Scientific control the collection of data recorded by the various field instruments described above. The data loggers trigger measurements of the desired variables at pre-designated times and store the acquired values. Next, TCom®, a software tool developed by Campbell Scientific Instruments, collects the stored data values from the data loggers and saves them in a central computer. An SNL program developed from Lab Windows/CVI completes the second stage; the program organizes the raw data files from the data loggers into arrays that correspond to the various plots. Finally, the data arrays are written into an Excel Workbook for final storage. Once the data is in final storage within the Excel Workbook the data can be more easily viewed and manipulated so that a thorough comparison of the measured data parameters among the project covers is possible.

\subsubsection{Stage 1: Data Collection - Data Logger}

Six Campbell Scientific Instruments- CR10 data loggers record and store the volumetric soil moisture contents measured by the TDR probes installed in the various soil layers of the test covers. A separate CR10 operates the on-site weather station; again, measurements of the different weather parameters (precipitation, relative humidity, 
incident solar radiation, wind speed and direction, and barometric pressure) are recorded and stored in the CR10. A CR7 data logger records and stores the other parameters such as surface runoff, soil temperature, lateral drainage, and percolation. Each of the data loggers operates independently with separate control programs.

Software code language known as Edlog $®$ developed by Campbell Scientific is used to write the programs that operate all of Campbell Scientific's data loggers. In Edlog, the user writes a .CSI file; the .CSI file contains a written description of all of the instructions that are to be transmitted to the computer, along with a description of the variables required for each instruction. When a .CSI file is complete, Edlog compiles the program and generates a .DLD file which is the actual program that is transmitted to the data loggers and controls their actions.

The .DLD programs are downloaded to the data loggers through the use of short haul RAD modems and Campbell Scientific software. All of the data loggers connect via RAD modems into communication ports on a central computer. The Tcom software allows the user to communicate with each of the data loggers from the one central computer location. Once connected to a data logger, the user can monitor the actions of the data logger, set the clock on the data logger, collect data, or transmit .DLD programs to the data logger as required in the first stage. Because each data logger is connected to a separate communication port, TCom is capable of sending different .DLD programs to the various data loggers.

Each of the data loggers used on the ALCD Project requires a different .DLD program. While six CR10's all use the same TDR recording instructions, the TDR system variables, such as cable length, within each instruction vary. Therefore, each of the six CR10's used for TDR measurements require a separate program. Each station is programmed to record the volumetric soil moisture content at each probe every two hours. Therefore, twelve TDR measurements per day are recorded from each probe. The .DLD program in the weather station's CR10 is programmed to record temperature, solar radiation, relative humidity, wind speed, wind direction, precipitation, and barometric pressure every ten seconds. However, the data are only stored and reported every hour. Therefore, the precipitation value stored each hour is the total amount of rain measured the previous hour. Also, only the maximum wind speed for the previous hour is recorded.

The program operating the CR7 is more sophisticated than the programs used to operate any of the CR10's. The CR7 program is divided into two tables. One of the tables measures and records the soil temperature from each of the soil thermocouples at the ALCD site every hour. The second table measures and stores the quantity of surface runoff, percolation, and lateral drainage (if applicable) from each of the covers. When the two tables operate simultaneously, the CR7 stores 24 measurements from each soil thermocouple and one measurement from each flow meter and tipping bucket. Once the appropriate programs have been downloaded, the data loggers will record and store the site data continuously. The stored values will remain in each data logger until the memory of each data logger has been exhausted, or the data is transferred into an external 
source. Project personnel transfer the data stored in data loggers on a regular basis to prevent losses that might result from memory overloading.

\subsubsection{Stage 2: Data Collection - Central Computer}

In the second stage, TCom software is used to collect the data from all of the data loggers and transfer it into the central computer's memory. Next, a program developed from Lab Windows/CVI separates the raw data collected by TCom into data files created for specific data, such as TDR data or weather station data. Within each data file, the data is separated into arrays that correspond to each of the six test covers; the arrays can then be viewed or manipulated with other programs developed from Lab Windows/CVI.

TCom begins the process of data collection. TCom is programmed to collect the data from all of the data loggers once a day between midnight and 1:00 a.m. Therefore, all data collected is a full-day's assemblage. TCom stores the data from each data logger in a separate data file. For example, the weather station data is stored in a file labeled "cr10_0.dat." The data stored in the CR10 files is in a raw form, therefore additional programs are used to reorganize the data into a more useful configuration.

The data in the files obtained by TCom can be viewed as a large two-dimensional data arrays in spreadsheet format. Rows represent specific times the measurements were taken and recorded and the columns contain data recorded from the various instruments (TDR probes, soil thermocouples, weather station, etc). For example, a typical TDR data file will contain twelve rows (hourly recordings) and as many columns as the number of TDR probes that particular data logger records. Because the imported raw data is in ASCIIcomma-separated format, and not in the form of the aforementioned two-dimensional array, a program developed from Lab Windows/CVI rewrites the data file into the twodimensional arrays. The reformatted data could then be used in other Lab Windows/CVI programs.

Before the new data arrays were created it was necessary to develop a data organizational scheme appropriate for the ALCD Project. In the case of the weather station data and the surface runoff, percolation, and lateral drainage data, the organizational scheme was fairly straightforward. The daily weather data is stored in two-dimensional array format. The surface runoff, percolation, and lateral drainage data are each stored in one-dimensional array as only one reading a day is recorded. The soil thermocouple and TDR data pose a different problem. The data for each of the covers is lumped together in the data files. For comparison purposes, the data is more useful if it can be viewed for each cover rather than one large array of data. Therefore, the thermocouple and TDR data are placed in twenty four arrays (twelve for thermocouple data and twelve for TDR data), with each array representing one of the 12 discrete plot segments being tested at the ALCD site (e.g., irrigated + non-irrigated segments of the six test covers). The programs developed from Lab Windows/CVI take the data files obtained by TCom and place the data in the desired scheme. 
The $\mathrm{C}$ functions that organize the data into the desired scheme all operate in a similar manner. First, the functions open the files collected from the data loggers by TCom. Once the files are opened, all of a single day's data is read into a large one-dimensional array and the file is closed. The one-dimensional array is broken into a two-dimensional array with the use of two loops. An inner loop copies the data from the one-dimensional array into the appropriate number of columns (i.e., the number of TDR probes) in the two-dimensional array configuration. Once the appropriate number of columns has been filled, the outer loop moves to the next row in the array and returns control to the inner loop; the inner loop fills the columns in the new row. The process continues until all of the data from the one-dimensional array is copied into the two-dimensional array. Finally, the completed two-dimensional array is saved as a new file with an appropriate name and date stamp (see Table 8.1).

The TDR data is stored in two separate files. One file consists of twelve two-dimensional arrays containing all of the records collected for each of the 12 discrete plot segments. A separate file contains twelve one-dimensional arrays containing the computed average daily volumetric soil moisture content in each plot segment. The average value stored in the data file is free from poor probe readings that might have been caused by external interference because any value that is outside of the range of $0-50 \%$ volumetric moisture content is not included in the calculation of the average; values beyond 50\% are not within the calibration range for the probes and, additionally, are not realistic for the soil materials at the ALCD site.

The data collected from the CR7 data logger is also separated into two files. The surface runoff, percolation, and lateral drainage are stored in a one-dimensional array onto one file. Soil thermocouple data is stored in twelve two-dimensional arrays in a different file.

The data files can be generated with two programs: "ALCD_tm.prj" or "ALCD_man.prj." "ALCD_tm.prj" operates on a 24-hr timer. The program will automatically open the data logger files, write the data into the new files and save the new files, and then delete the old data logger files. If the data must be seen immediately and the timer has not created the data files, a user may obtain the data files with "ALCD_man.prj." "ALCD_man.prj" performs the same functions as "ALCD_tm.prj;" however, are not created automatically on a timed sequence and the user must execute a prompt in the program for the data file creation.

Table 8.1. Data File Names

\begin{tabular}{cc}
\hline Data collected & $\begin{array}{c}\text { Name of data file if collected } \\
\text { on March 31 }\end{array}$ \\
\hline Weather Station & wea3_31 \\
TDR & tdr3_31 \\
Average TDR & tavg3_31 \\
Thermocouple & tcp3_31 \\
Surface Runoff, Percolation, and & cr73_31 \\
Lateral Drainage & \\
\hline
\end{tabular}


Once the data files have been created, the data is accessible with other $\mathrm{C}$ programs developed from Lab Windows/CVI. For example, a user may view and produce hard copies of all of the data with "ALCD_mn.prj." "ALCD_mn.prj" provides panels that readily allow a user to view the TDR data (daily average or two hour records), the weather station records, hydrological data (surface runoff, percolation, lateral drainage), and graphs of soil temperature recorded throughout the day. The program allows site personnel to identify any problems that might occur with the various instrumentation. However, the program does not allow for recorded data values to be manipulated or used directly for simple calculations. Therefore, another program is required to place the data in an easily manageable format.

\subsubsection{Stage 3: Data Transfer}

The final step in the data acquisition process is to place the data in a format that allows easy manipulation for comparison of the test covers. In such a format, the pertinent data for all of the covers must be easily viewed. Also, the data should be accessible for edits and simple calculations. For ALCD purposes, the data for each of the twelve plot segments is written in a separate spreadsheet. The twelve separate spreadsheets are saved in two Excel Workbooks. In such a format, the data for each plot segment file remains separate. However, the data between and among the 12 plot segments can be visually compared by switching between the desired worksheets within the workbook itself. Furthermore, data from the different plot segments may be copied and pasted onto new worksheets within the workbook where subsequent data calculations may be performed. Therefore, the ability for simple visual data comparisons and comparisons based upon statistical calculations (means, standard deviations, etc.) makes an Excel Workbook the ideal format for final data storage.

Another program developed from Lab Windows/CVI writes the data into the Excel Workbooks. "Exc_dat.prj" allows a user to open the Excel Workbook "plots.xls", write the new data from each plot into the appropriate worksheet, and save the workbook with a new name. First, the user accesses the Excel program from "exc_dat.prj" and then opens "plots.xls." "Plots.xls" contains twelve spreadsheets, one for each of the 12 test plot segments. Each spreadsheet contains cells labeled specifically for the data associated with each plot segment; the spreadsheets also have formulas to average and report the moisture content of each of the different soil layers in the various plot segments. Next, the user extracts the desired data. In extracting the data, two options are available. The user may allow "exc_dat.prj" to automatically open the current day's data files, or the user may manually select any of the existing data files. Once the data has been extracted from the data files, the user prompts "exc_dat.prj" to write the data into each plot's respective spreadsheet. When all of the data has been written into the new workbook, the user assigns the workbook a new name prior to saving so that "plots.xls" remains empty for future use. Two options exist in saving the file: 1) the user may allow "exc_dat.prj" to save the file with a title containing the current date; for example, a file saved on June 2 would be saved as "dat6_2.xls.", or 2) the user may assign a different name to file. 
Once the workbook has been saved, "exc_dat.prj" closes the workbook and the user is then allowed to repeat the entire process by entering additional data into a new workbook.

"ALCD_row_data.prj" operates in a similar manner as "exc_dat.prj." Once the user accesses the Excel program, he/she is allowed to open the file "alcdwb.xls." "Alcdwb.xls" contains 12 worksheets, each corresponding to one of the 12 plot segments. The user has similar options for obtaining the data as in "exc_dat.prj." However, the procedure for placing data within the workbook is different. The program writes all of the data from one plot onto a row corresponding to its Julian day on the plot's worksheet. In this format, a user may observe how one TDR probe or other instrument reading changes from day to day within the plot segment. Once all of the data has been entered the files are all saved under the same name; therefore, all of the data is stored in one easily accessible file. The data can now be easily viewed and manipulated for any calculations required for the plot segment comparisons. With data collection and organization complete, the analyses needed to compare the various plot segments become less difficult. Therefore, time resources can be directed towards other project activities.

\subsection{DATA ANALYSIS AND INTERPRETATION}

The objectives of the data analysis are to: (1) summarize, compare, and document water balance data and to quantify potential benefits of the different cover designs in the ALCD, (2) compare test results with predictive models, and (3) document recommendations on use of cover alternatives for interim stabilization and/or closure of landfills in arid/semiarid regions.

\subsection{Water Balance Data}

Hydrologic monitoring data will be used to compute a daily water balance (see Equation 3.42) for each cover design as a means of determining the performance of the cover. Analysis of variance techniques will be used to test for statistically significant differences in water balance components among the various designs and as a function of precipitation (i.e., comparisons of irrigated vs. non-irrigated plot segments. Special emphasis will be placed on evaluating the barrier alternatives with emphasis on technical performance, ease of construction, and cost. Variables that will be quantitatively or qualitatively compared include the following:

- Runoff

- Erosion

- Changes in soil moisture

- Interflow or interflow plus water vapor removal

- Percolation/Leaching

- Evapotranspiration

- Vegetation Parameters (percent ground cover, biomass production, and species composition) 
- Ease of Construction

- Cost

Important components of this sub-task include the following:

- generating final summary data in required format (i.e. hard copy, disk, etc.),

- computing periodic water balance,

- conducting statistical analysis, and

- generating interim reports on results.

\subsection{Comparison with Predictive Models}

Results from the demonstration will be compared with those generated by predictive models such as the Hydrologic Evaluation of Landfill Performance (HELP) Model for Phase I covers. Important components of this sub-task include the following:

- Running HELP for ALCD cover designs;

- Formatting monitoring data for comparison with HELP output;

- Conducting statistical tests for differences between data generated by HELP and field data;

- Documenting results of comparisons; and

- Submitting recommendations and results to the HELP model owners.

The project's field results will also be compared with predictions produced by the TOUGH2 program. TOUGH2 is an unsaturated flow software package deemed to best represent the movement of water through the test covers under unsaturated conditions (Webb, 1996).

\subsection{Documentation}

Dissemination of ALCD Project results are planned through presentations at briefings, technical and operational meetings, and symposia, reports, peer reviewed publications, and possibly book chapters. Important components of this project sub-task include the following:

- publication/presentation responsibilities,

- publication/presentations coordinator,

- preparing individual reports from participants,

- integration of individual reports,

- peer review of reports,

- identifying publication vehicles, and

- publishing individual/integrated papers. 


\subsection{DATA QUALITY ASSURANCE}

The Data Management System for the ALCD is organized in a Notebook System containing all documentation relevant to the test, including electronic data. Access to the Notebook System is controlled to protect the data.

The Data Management System is a key element of the ALCD Project. The goals of the Data Management System are to: (1) ensure all relevant documentation is collected and stored in a trackable manner, (2) ensure compliance with Quality Assurance requirements, and (3) control data dissemination. A comprehensive Data Management System is particularly important for a long-term project such as the ALCD because of the likely probability of changes in project personnel during the course of the project.

The Notebook System contains all documentation relevant to the project. A partial listing of the Notebook System components is as follows:

- Test Plan

- Instrumentation

- Procurement records

- Calibration records

- Installation procedures and records

- As-built configuration

- Dimensions of each design

- Instrumentation location

- Construction notes

- Photographs

- Data collection procedures

- Erosion

- Runoff

- Breakthrough and lateral divergence

- Met station

- Hydrologic testing

- Vegetation surveys

- Other

- Soil collection and testing

- Soil sampling procedures

- Soil testing procedures

- Soil testing results 
- Manual data (raw)

- Erosion

- Vegetation

- Breakthrough, lateral divergence

- Stress

- FDR (backup, if needed)

- Hydrologic testing

- Other

- Electronic data (raw)

- Met station

- TDR

- Other

- Water balance database (screened)

A series of spreadsheets are used to store the electronic data as described in Section 8. Copies of the spreadsheets are submitted to the Notebook System.

Data for all of the water balance components, including both electronic and manual data, are combined in a water balance database. These data are screened to remove faulty data, and to allow its assemblage into a common time scale. The water balance database is updated periodically and stored in the Notebook System.

Only that data contained in the Notebook System is to be considered official for dissemination purposes. The use of unofficial data is not permitted, except as specifically authorized by the Project Manager or his/her designated delegate. Access to the Notebook System is restricted to prevent loss of data. Housing the Notebook System in Building 823 allows for sufficient control and facilitates access by clerical personnel.

\subsection{ENVIRONMENTAL SAFETY \& HEALTH REQUIREMENTS}

\subsection{ES\&H Program Requirements}

Environmental, Safety, and Health $(\mathrm{ES} \& H)$ requirements and concerns are taken very seriously for all work performed on the ALCD Project. All personnel involved in the ALCD are required to undergo proper ES\&H training for their specific activities. Any project participant that is required to use any potentially hazardous equipment or materials on the ALCD site must receive specific training for their appropriate and safe use. Each phase of ES\&H training must be documented, with a copy being kept in the Department $6131 \mathrm{ES} \& \mathrm{H}$ file. 
SNL's ES\&H Manual is enforced for the project. Everyone working on the project must be familiar with all the components of this manual. All personnel involved with ALCD field activities must attend a safety briefing prior to the start of their activities.

Periodically, a brief on-site safety meeting will be conducted during the morning prior to the commencement of day's field activities. This safety meeting will summarize all potential hazards/problems anticipated during the day and outline the recommended remedies for these problems in the event that they are encountered. The project participants are also notified at this time of any ES\&H violations that have been sighted.

\subsection{ES\&H Required Permits}

Permits and related activities required for field activities at the ALCD Project site:

- NEPA Permit

- Topsoil Disturbance Permit

- Dust Control Plan

- Notice of Intent to Discharge Surface Water

- DOE Safety Documentation Form

- Excavation Permit

All of the above permit requests were submitted by the appropriate project authority and subsequently approved.

\subsection{EXPECTED BENEFITS OF THE ALCD}

The anticipated outcome of this demonstration project will be a thorough and critical evaluation of the various cover designs tested and a recommendation for the acceptance of alternative cover designs for landfills in the arid/semiarid western United States to the appropriate regulatory agencies. The intent is to widely publish and disseminate the study results so as to present the advantages/limitations of each of the cover designs tested to the public, and as many regulatory personnel and design engineers as possible.

The demonstration is expected to provide performance and cost data for alternative cover components and systems that are more applicable to the drier Western climatic conditions. A direct comparison between conventional (based on more humid climatic conditions) and alternative designs will be available. The "active" testing phase of the project will permit data to be collected under extreme and accelerated conditions. This information will provide those individuals responsible for the development of landfill cover design guidance to have a defensible basis for the transition from designs suited for the humid Eastern U.S. to other designs more appropriately suited to the drier Western states.

The probable outcome of this demonstration project is the acceptance of alternative cover designs that are significantly less costly to implement than conventional designs. Given the thousands of 
acres of buried waste sites across the $\mathrm{DOE}$ and $\mathrm{DoD}$ complexes to that need to be stabilized and isolated, the payoff from this demonstration may be on the order of many hundreds of millions of dollars in savings to U.S. tax-payers.

\subsection{Literature Cited}

Benson, C.H. and M.V. Khire. 1995. Earthen covers for semi-arid and arid climates. Pages 201217 in R.J. Dunn and U.P. Singh, eds. Environmental protection and landfill covers...environmental protection and land recovery. ASCE, San Diego, CA.

Boyton, S.S. and D.E. Daniel. 1985. Hydraulic conductivity tests on compacted clay. Journal of Geotechnical Engineering. Vol. 111, No.: pp. 465-478.

Claymax 1995. Material Specifications Claymax Geosynthetic Clay Liners: CLAYMAX 200R, CLAYMAX 500SP, CLAYMAX 506SP. Claymax Corporation, Fairmount, GA 30139, s9200, January 1995.

EPA. 1994. Construction Quality Assurance/Construction Quality Control (CQA/CQC) for Waste Containment Facilities; and Hydrologic Evaluation of Landfill Performance (HELP) Model. EPA/625/K-94/001, May 1994. Part I. Geomembrane liners. Geotextiles and Geomembranes. Vol. 8:27-67.

EPA. 1994. The Hydrologic Evaluation of Landfill Performance (HELP) Model, Engineering Documentation for Version 3. EPA/600/R-94/168b. September 1994.

EPA. 1991. EPA Seminar Publication. Design and Construction of RCRA / CERCLA Final Covers. EPA/625/4-91/025, May 1991.

EPA. 1988. Draft - Background Document. Case Studies on Ground-Water and Surface Water Contamination from Municipal Solid Waste Landfills: Criteria for Municipal Solid Waste Landfills, (40 CFR Part 258), Subtitle D of Resource Conservation and Recovery Act (RCRA), U.S. Environmental Protection Agency, Office of Solid Waste. EPA/530-SW-88040. (PB88-242466), July 1988.

Fairbridge, R.W. and C.W. Finkl, Jr. 1979. The Encyclopedia of Soil Science, Part 1 - Physics, Chemistry, Biology, Fertility, and Technology. Dowden, Hutchinson \& Ross, Inc. Stoudsburg, PA. 646 p.

Hakonson, T.E.. 1996. The Use of HELP3 for Design and Evaluation of Some Cover Alternatives at the ALCD, CSU-DRHS-SNL-ALCD-96-1, Colorado State University, October 1996.

Melchoir, S., K. Berger, B. Vielhaber, and G. Miehlich. 1994. Multilayered landfill covers: field data on the water balance and liner performance. Pages 411-425 in G.W. Gee and N.R. Wing, Eds., In-Situ Remediation: Scientific Basis for Current and Future Technologies, $33^{\text {rd }}$ Hanford Symposium on Health and the Environment, Pasco, WA.

Nyhan, J. W., and L. J. Lane. 1986. Erosion control technology: a user's guide to the use of the universal soil loss equation at waste burial sites. Los Alamos National Laboratory Report, LA-10262-M.

Nyhan, J. W., T. E. Hakonson, and B. J. Drennon 1990. A water balance study of two landfill cover designs for semiarid regions. Journal of Environmental Quality: 281-288. 
Pruess, K. 1991. TOUGH2 - A General Purpose Numerical Simulator for Multiphase Fluid and Heat Flow, LBL-29400, Lawrence Berkeley Laboratory, May 1991.

Webb, S.W. 1996. "Selection of a Numerical Unsaturated Flow Code for Tilted Capillary Barrier Performance Evaluation." Sandia National Laboratories report - SAND96-2271

Wentz, Charles A. 1989. Hazardous Waste Management. McGraw-Hill Publishing Company, New York, NY 10020.

US Department of Energy - Office of Environmental Guidance 1993. RCRA Information Brief "Closure of Municipal Solid Waste Landfills (MSWLFs)". EH-231-036/0793. 


\section{ATTACHMENT A \\ Soil Amendment Study Implementation \\ and Procedures}

\section{Site Preparation}

The site selected for the soil amendment study, a triangularly shaped area of approximately 2.0 acres, is located just east of the existing ALCD Phase I and II Landfill Covers. The existing native vegetation (arid rangeland grasses and shrubs) within the study site was scraped away and the top soil (upper $1.0 \mathrm{ft}$. of soil) was then graded to one corner of the site where it was temporarily stock-piled. The underlying soil was then mechanically "ripped" so as to destroy the natural soil structure within the next depth interval (12 to 24 in. depth). The stockpiled soil was subsequently spread back uniformly onto the site. The cumulative effect of the vegetation and topsoil removal and ripping of the subsoil was to simulate site disturbance during a landfill excavation and the subsequent regrading of the topsoil material over the landfill cover after closure.

\section{Plot Establishment}

Twenty-four study plots were then established to evaluate the effects of the various soil amendments on subsequent plant establishment (Figure A-1). The layout of the plots (each plot is $100 \mathrm{~m}^{2}$ in area, dimensions $10 \times 10 \mathrm{~m}$ ) within the triangularly shaped area included approximately $3.0 \mathrm{~m}$ buffer strips between each plot. Three organic soil amendments (biosolids, dairy manure, and green waste composts) and a cobble mulch (2-3 inch diameter cobbles) were selected for the study. The green waste and biosolids compost were donated by the City of Albuquerque's Public Work's Wastewater Utility Division. The diary manure compost and cobbles were purchased locally from private industry, Western Organics, Inc. and Western Mobile, respectively. Either $2.5 \mathrm{yd}^{3}$ or $5.0 \mathrm{yd}^{3}$ (depending upon the treatment) of organic amendment was applied to each plot with a front-end loader ( $0.5 \mathrm{yd}^{3}$ bucket), spread uniformly over the entire plot, and then rotor-tilled to a depth of about six inches. The plots were then seeded with a mixture of cool and warm season native grassland species (Table A-1). On the three cobble-mulched plots, the seed mixture was broadcast uniformly over the ground surface and the cobbles were then spread uniformly over the plots to completely cover ground with a mono-layer of cobbles.

\section{Soil Sampling}

Three soil samples were obtained from random sites within each plot to establish a baseline on soil chemical properties prior to the addition of the organic amendments and cobble mulch. Each soil sample (approximately $1.0 \mathrm{~kg}$ ) was taken from the upper six inches of soil. The samples were placed in air tight, quart capacity zip-lock plastic bags and transported to a greenhouse and allowed to dry to the dry greenhouse air moisture conditions. The soil samples were passed through a $2 \mathrm{~mm}(\# 10)$ sieve to separate the fine earth fraction $(\cdot 2.0 \mathrm{~mm}$ diameter particles) from coarse fragments ( $>2.0 \mathrm{~mm}$ diameter). The fine earth fraction was then submitted to New Mexico State University's Soil Water and Air Testing Laboratory for analyses. 
Table A-1. Species selected for revegetation of the Soil Amendment Study Plots.

\begin{tabular}{lcc}
\hline Warm Season Grasses: & $\begin{array}{c}\text { Desired Establishment } \\
\text { (\% of total vegetation) }\end{array}$ & $\begin{array}{c}\text { Quantity in Mixture" } \\
\text { (lbs/acre) }\end{array}$ \\
\hline Bouteloua gracillis (Blue Grama) & 20 & 1.0 \\
$\quad$ Hilaria jamesii (Galleta) & 10 & 3.0 \\
$\quad$ Sporabolis cyptandrus (Sand Dropseed) & 50 & 0.5 \\
Cool Season Grasses: & 10 & 3.0 \\
$\quad$ Oryzopsis hymenoides (Indian Ricegrass) & 10 & 4.0 \\
Stipa comata (Needle \& Thread) & & \\
\hline
\end{tabular}

\footnotetext{
*Approximate percentage of total species present in number of plants per given area.

"Note that differences in weight among the various species can result in large differences in the mass ratio (lbs/acre) of seed required in the seed mixture.
}

\section{Laboratory Analyses}

Analytical tests for the soils followed standard procedures as outlined in Page et al. (1982) and Richards (1969). Soil samples were analyzed for $\mathrm{pH}$, electrical conductivity (EC), \% organic matter, total nitrogen (TKN), $\mathrm{KCl}$ extractable $\mathrm{NH}_{4}-\mathrm{N}$ and $\mathrm{NO}_{3}-\mathrm{N}, \mathrm{NaHCO}_{3}$ extractable $\mathrm{P}$, total phosphorus, and heavy metal contents $(\mathrm{Cd}, \mathrm{Cu}, \mathrm{Pb}, \mathrm{Zn}$, and $\mathrm{Mo}$ ). In addition, we conducted a textural analysis (hydrometer method, Day (1982)) of the soils during the first sampling period. Saturated pastes were used for EC and $\mathrm{pH}$ measurements. Organic matter contents were determined by chromic acid oxidation using the modified Walkley-Black method described by Nelson and Sommers (1975). We determined total nitrogen on Kjeldahl digests (Bremner and Mulvaney 1982). $\mathrm{NaHCO}_{3}$ extractable $\mathrm{P}$, an index of plant-available $\mathrm{P}$ in alkaline soils, was analyzed following the methodology outlined by Olsen and Sommers (1982) as. $\mathrm{KCl}$ extractable $\mathrm{NH}_{4}-\mathrm{N}$ and $\mathrm{NO}_{3}-\mathrm{N}$ were determined colorimetrically following the procedures outlined by Bremner (1965). Total phosphorus was determined colorimetrically in extracts from total digests of soil samples according to the method of Murphy and Riley (1962). Similarly, metal contents were determined from extracts of total soil digests by optical emission spectrometry (Soltenpour et al. 1982). 
Fence

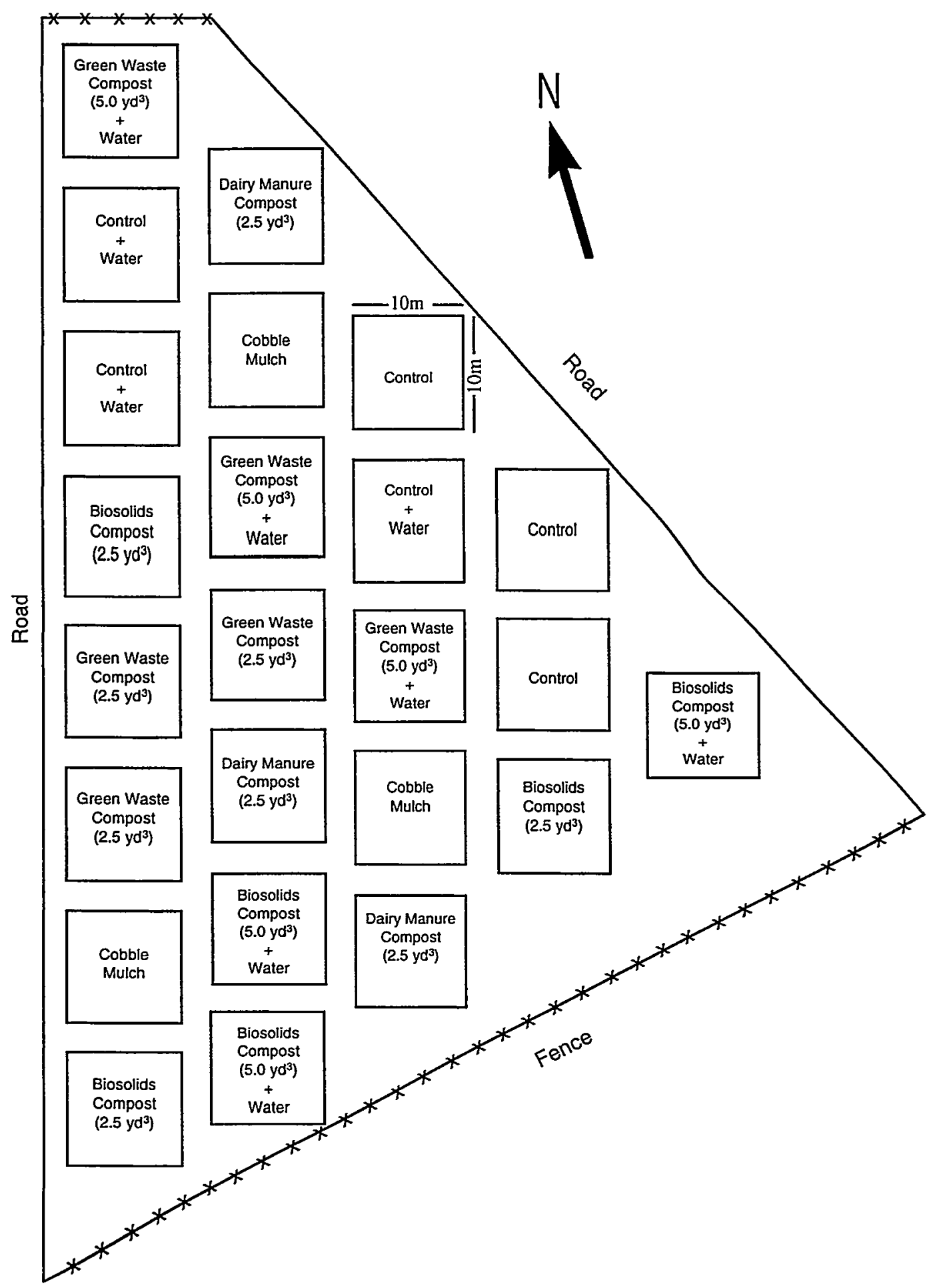

Figure A-1. Soil Amendment Study plot layout at the ALCD Project Site. [diagrammatic only - not to scale] 


\section{Statistical Analysis}

Analysis of variance (ANOVA) techniques will be used to test for statistically significant differences between treated and control plots in plant cover and nutrient and trace elements in soils. Repeated measures ANOVA (Statistical Package for the Social Sciences) will be used to test for significant main factors (treatment and time), significant interactions, normally distributed data, and homoscedasticity (homogeneous variances). If the data are normally distributed and have homogeneous variance, we will proceed with a one-way ANOVA followed by a Tukey's multiple comparison test. If we encounter problems with non-normality or heteroscedasticity we will then employ the Pillai's statistic which is "more robust" to these problems than the F statistic. We will then proceeded with a one-way ANOVA and the appropriate multiple comparison test. Unless otherwise noted, we will adapt a type I error rate of $\alpha=0.05$ for all statistical analyses.

\section{Literature Cited}

Bremner, J.M., and C.S. Mulvaney. 1982. Nitrogen-Total. In: A.L. Page et al. (ed.) Methods of Soil Analysis. Part 2 - Chemical and Microbiological Properties. 2nd ed. Agronomy 9:595-624. American Society of Agronomy, Madison, WI.

Day, P.R. 1982. Particle fractionation and particle-size analysis. pp. 545-567. In: A.L. Page et al. (ed.) Methods of Soil Analysis. Part 1 - Physical and Mineralogical Properties, Including Statistics of Measurement and Sampling. 2nd ed. Agronomy 9:595-624. American Society of Agronomy, Madison, WI.

Murphy, J., and J. P. Riley. 1962. A modified single solution method for determination of phosphate in natural water. Anal. Chim. Acta. 27:31-36.

Nelson, D.W. and L.E. Sommers. 1975. A rapid and accurate procedure for estimation of organic carbon in soil. Proceedings of the Indiana Academy of Sciences 84:456-462.

Olsen, S.R. and L.E. Sommers. 1982. Phosphorus. pp. 403-430. In: A.L. Page (ed.) Methods of Soil Analysis, Part 2 - Chemical and Microbiological Properties. 2nd ed. Agronomy 9:403-430. American Society of Agronomy, Madison, WI.

Richards, L.A. (ed.) 1969. Diagnosis and improvement of saline and alkali soils. USDA Agric. Handbk. 60, U.S. Department of Agriculture, Washington, D.C.

Soltanpour, P.N., J. Benton Jones, Jr., and S.M. Workman. 1982. Optical emission spectrometry. pp. 29-65. In: A.L. Page (ed.) Methods of Soil Analysis, Part 2 Chemical and Microbiological Properties. 2nd ed. Agronomy 9:403-430. American Society of Agronomy, Madison, WI. 


\section{ATTACHMENT B}

Phase I - Drawings 


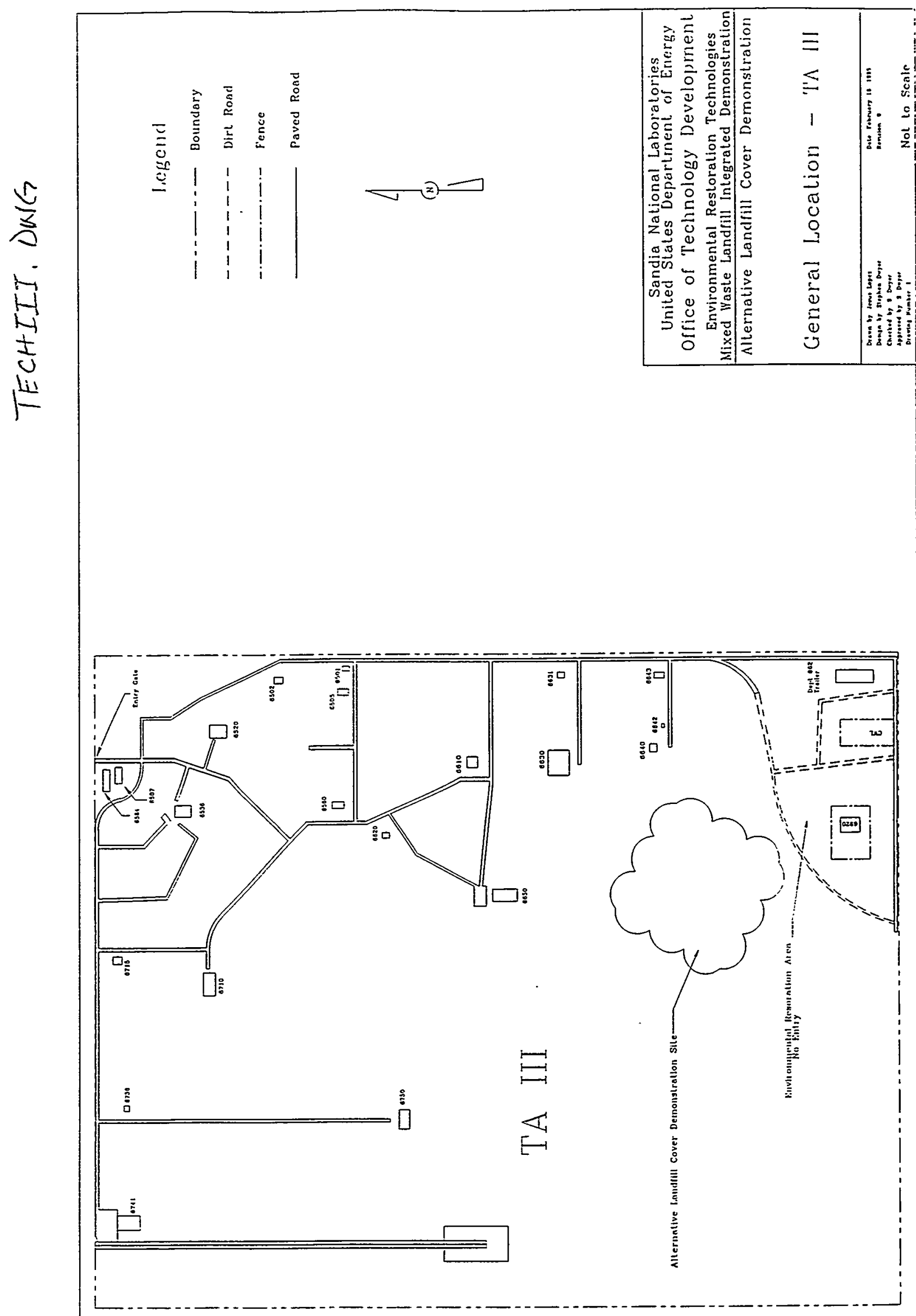



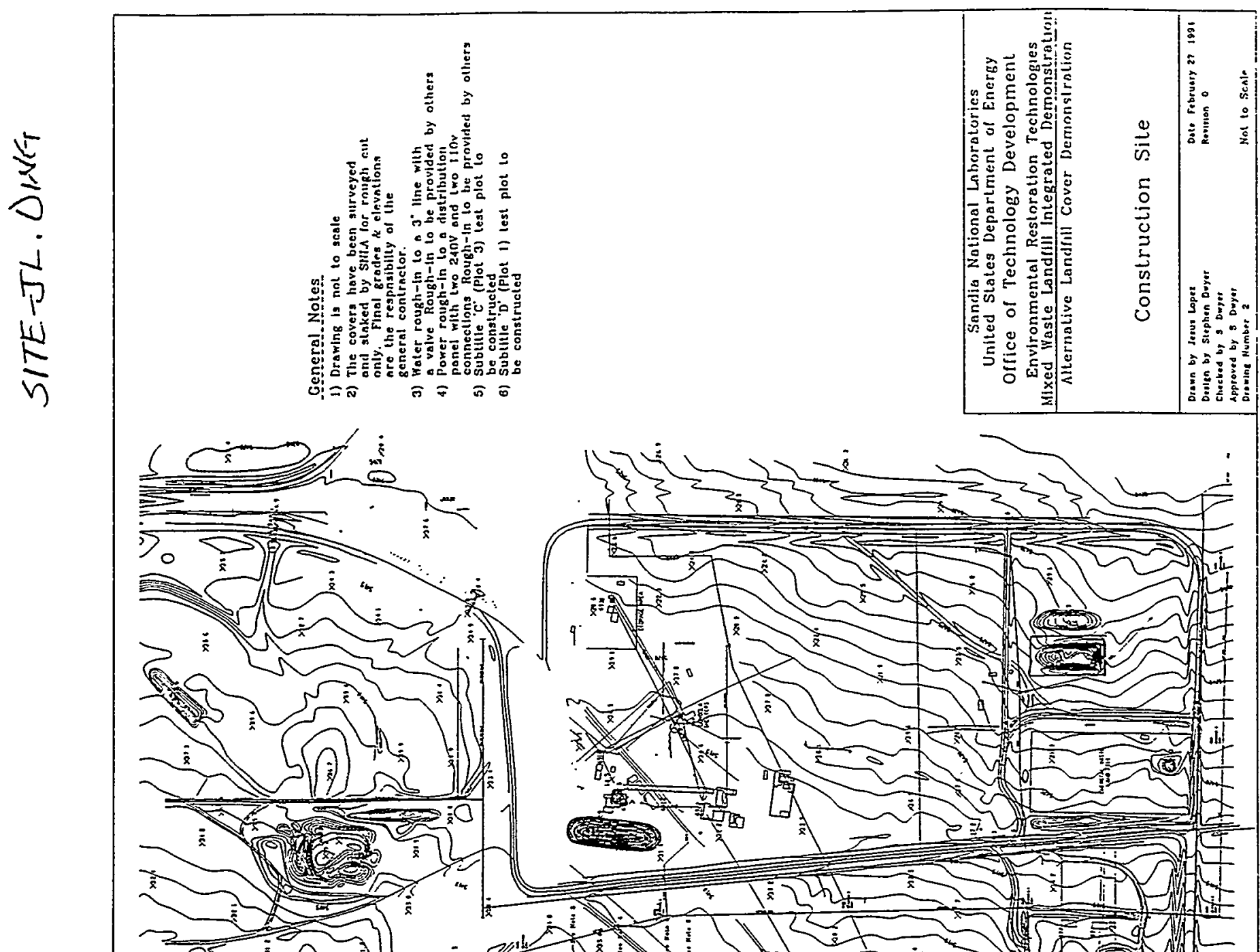


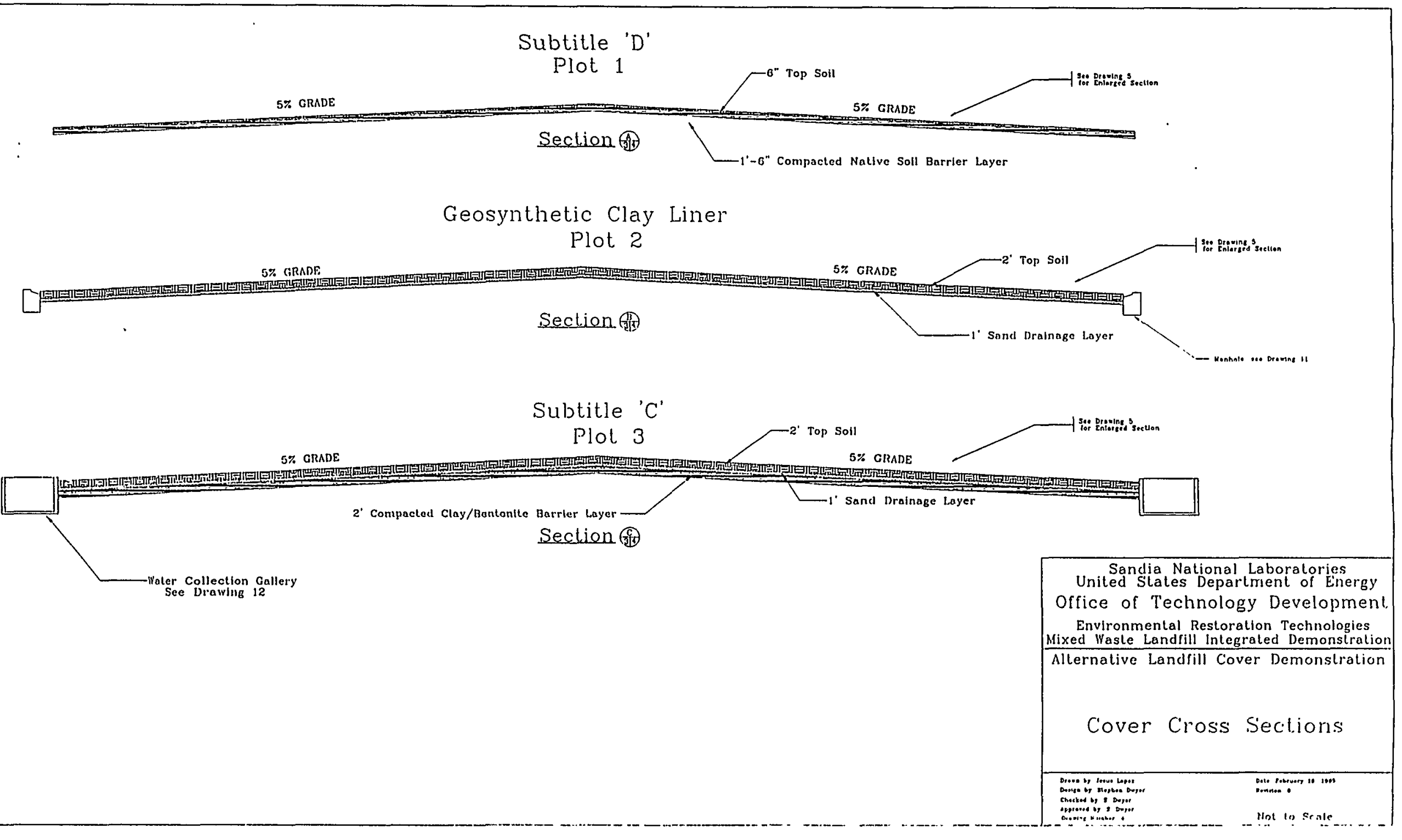




\section{Geosynthelic Clay Liner Cover}

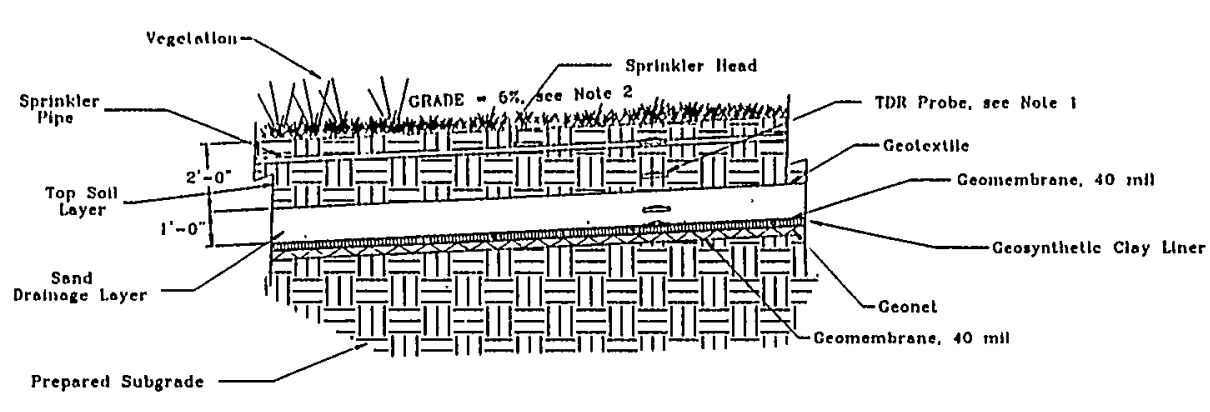

\section{Subtille 'C' RCRA Cover}

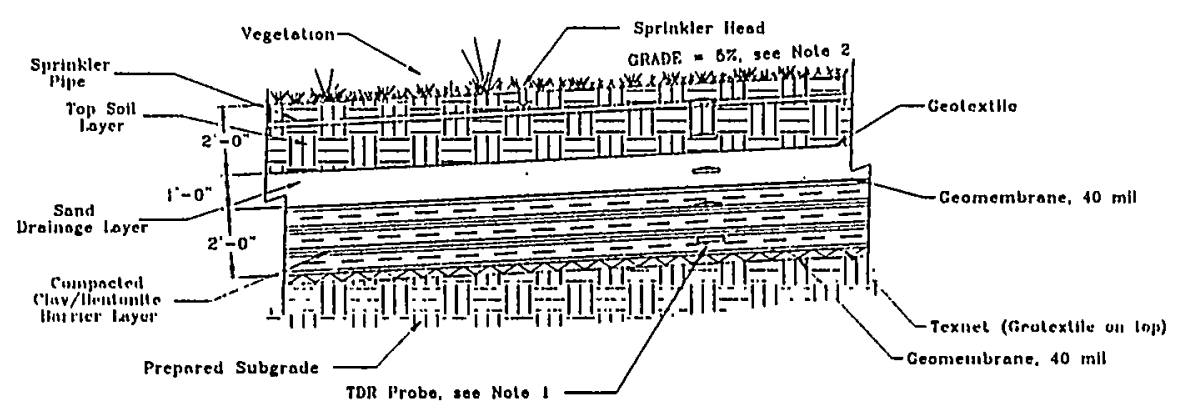

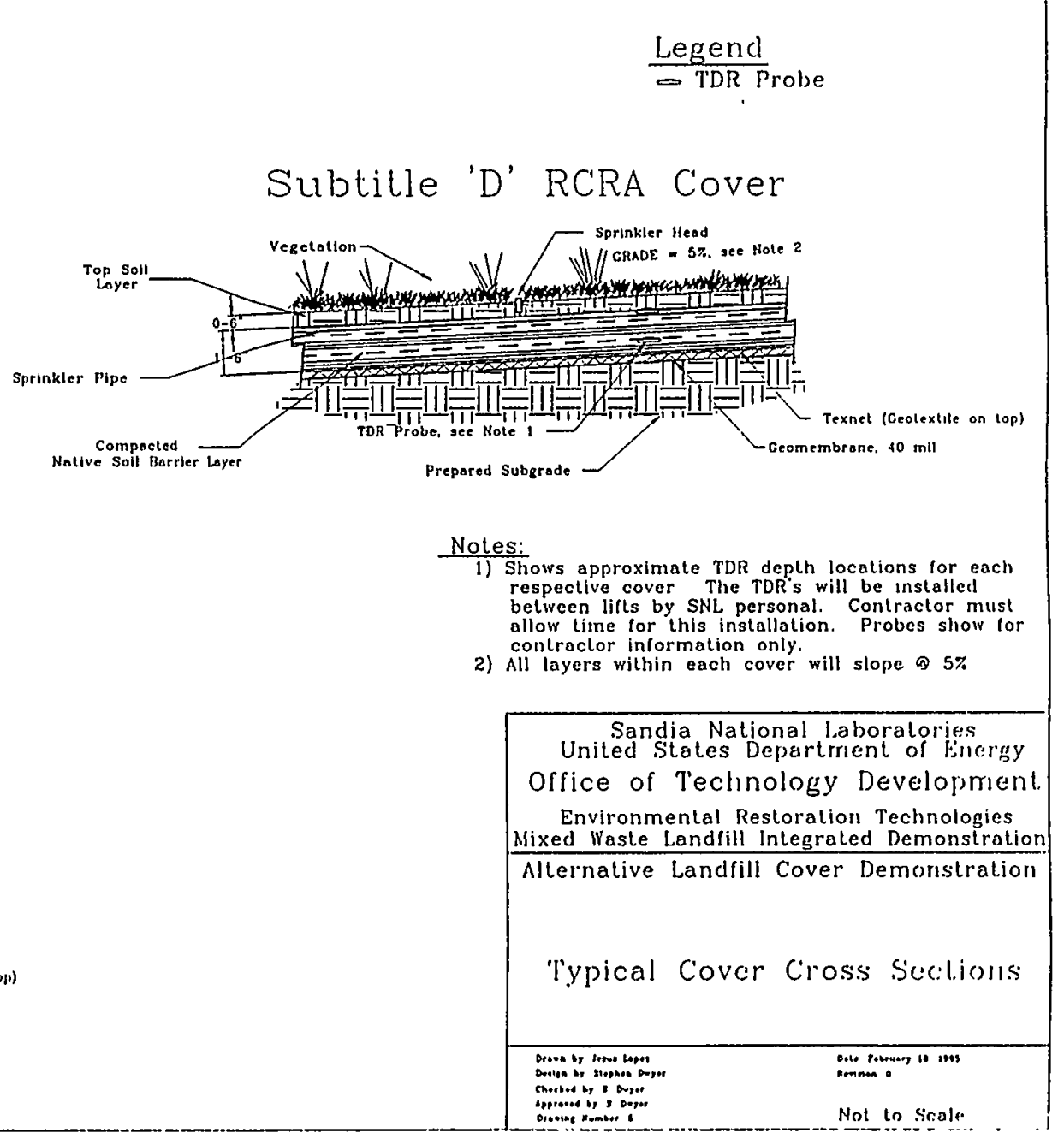




\section{INST. DWG}

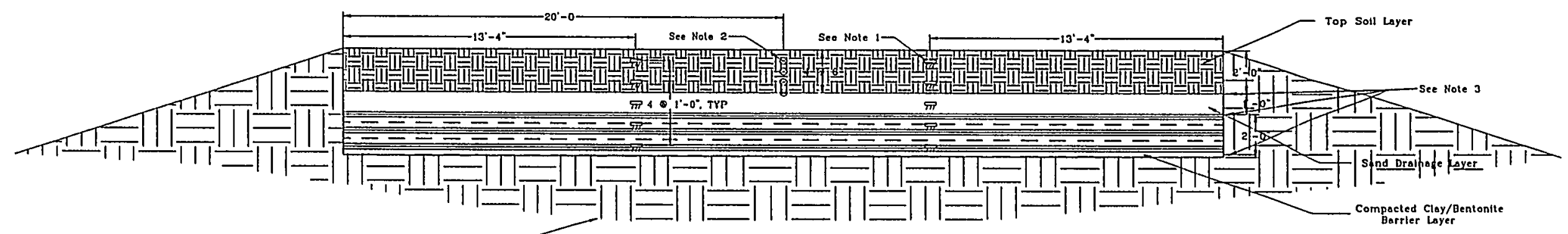

Prepared Subgrade $\sim$

\section{Subtitle 'C' RCRA Cover (Plot 3) Seclion (중}

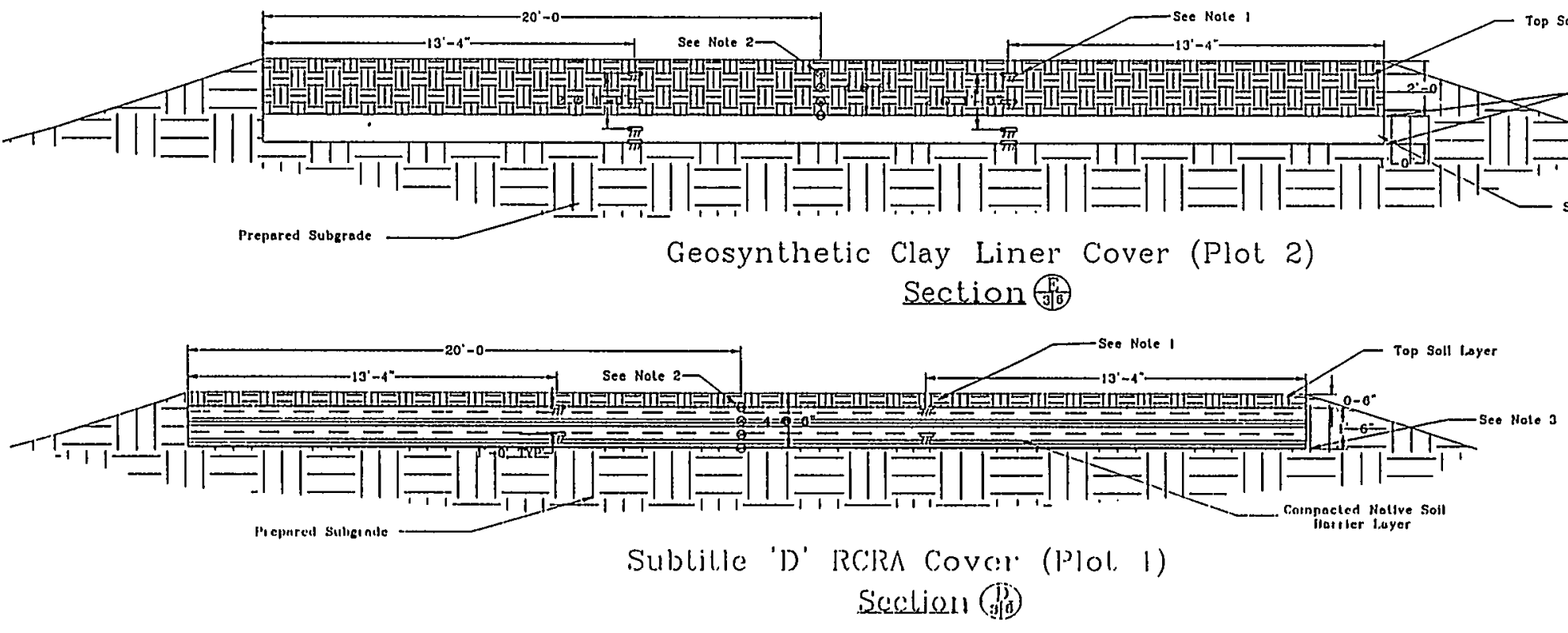

\author{
Legend \\ in TDR Probe \\ - Thermocouple
}

Netes:

Stiows approximate instrumentation The TDR's will be installed belween installed by SNL personal Contraclor must nllow lime for tors inslallation. Probes shown

2) Thermocouples will be inslalled ust ollow time for this installation They will be installed in llie lop 2
of the cover shown for iniormation
of

3) All geosynthetics are to stop at the respective cover They will in no cose slop short of this point. but than a couple of inches beyond the edge.

Sandia National Laboratories
United States Department of Fneroy Office of Technology Developinent Environmental Restoration Technologies Mixed Waste Landill Integrated Demonsiration Nlternative Landfill Cover Demonstration

Typical Cross Sections

\begin{tabular}{|c|c|}
\hline 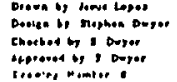 & 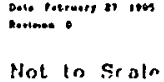 \\
\hline
\end{tabular}




\section{PIPE-FR, DWG}

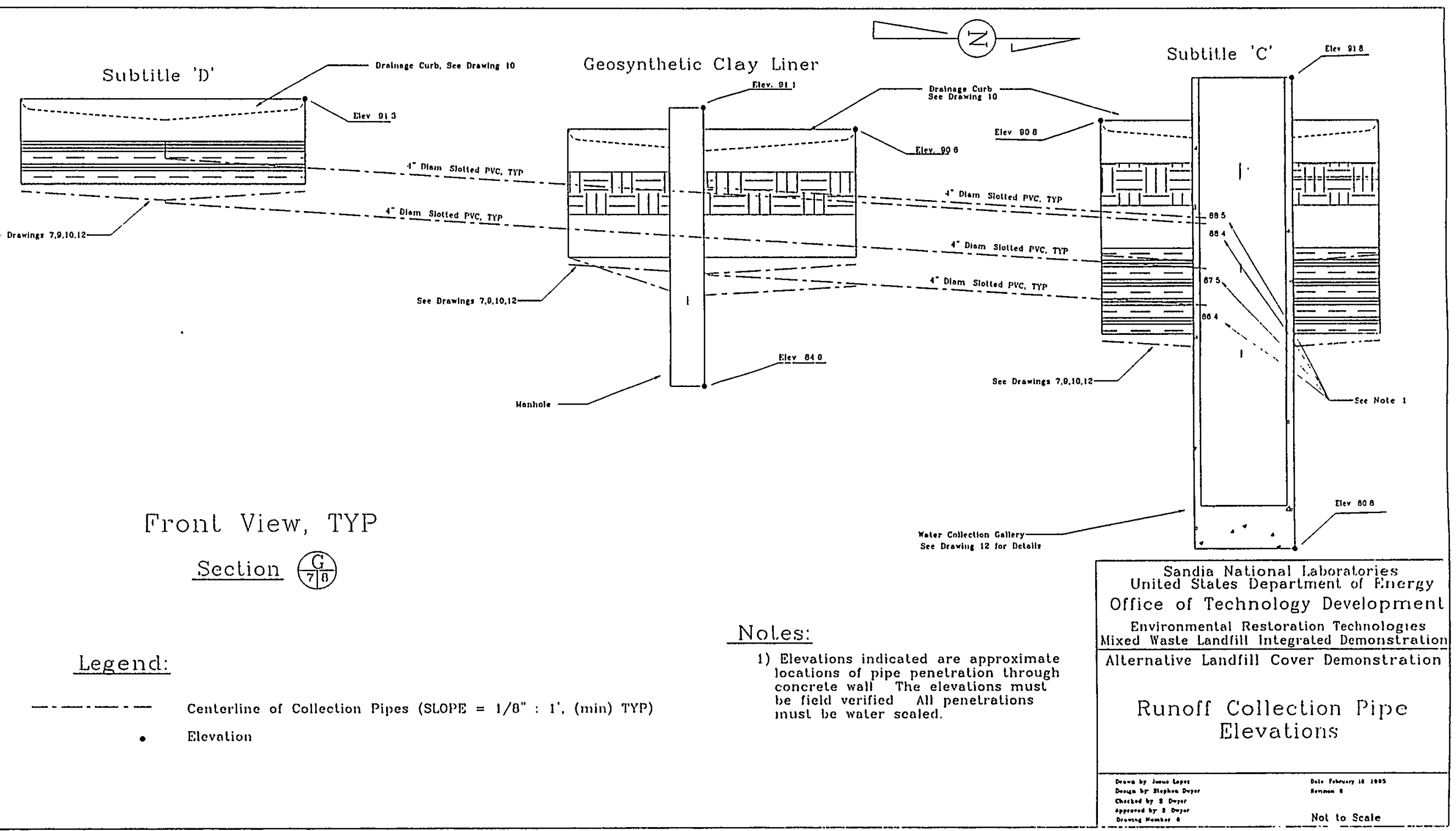




\section{PIPCOL. DNG}

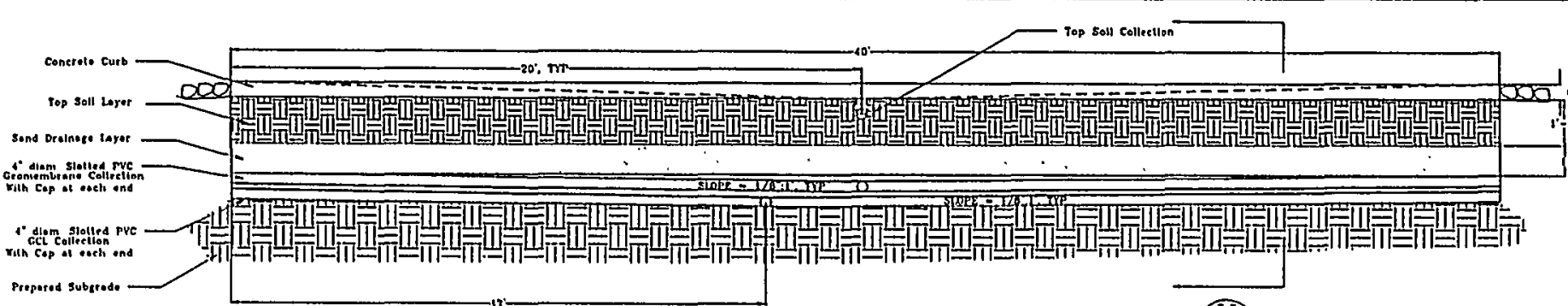

propored suberode

\section{Geosynthetic Clay Liner} Section $(\sqrt{3})$
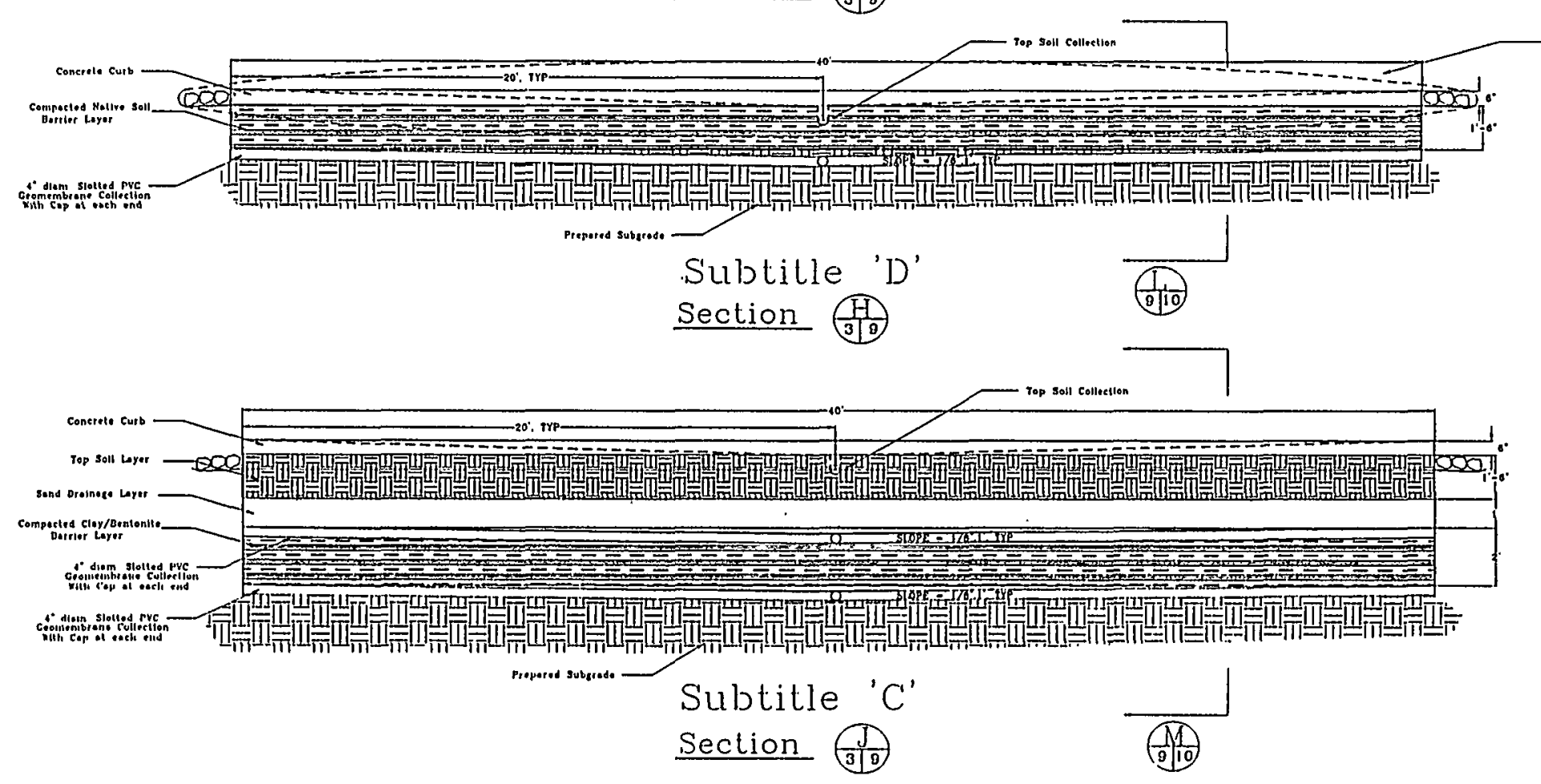

United States Department of Energ Office of Technology Development Environmental Resloration Technologics
Mixed Waste Landill Mixed Waste Landfill integrated Demonstration Alternative Landfill Cover Demonstration

Runoff C'ollection Pipes 


\section{PJPE-SI, DWG}

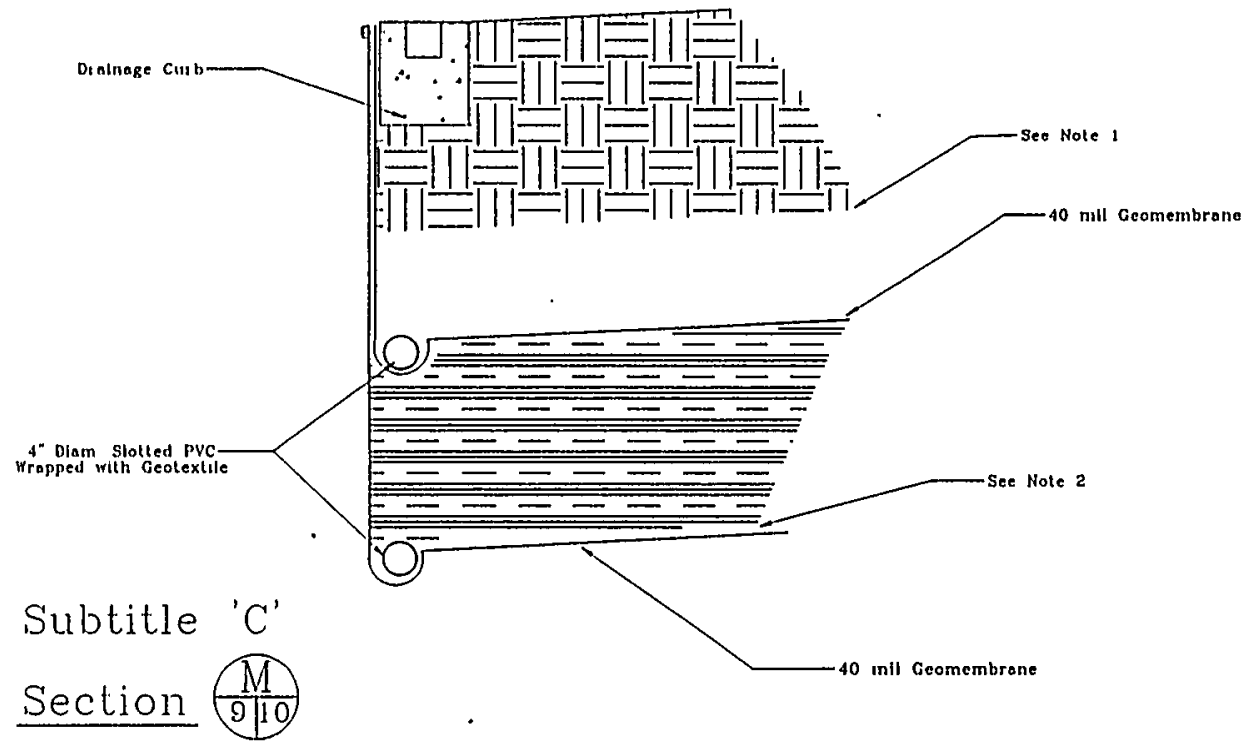

Geosynthetic Clay Liner section $(\overline{0})$

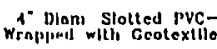

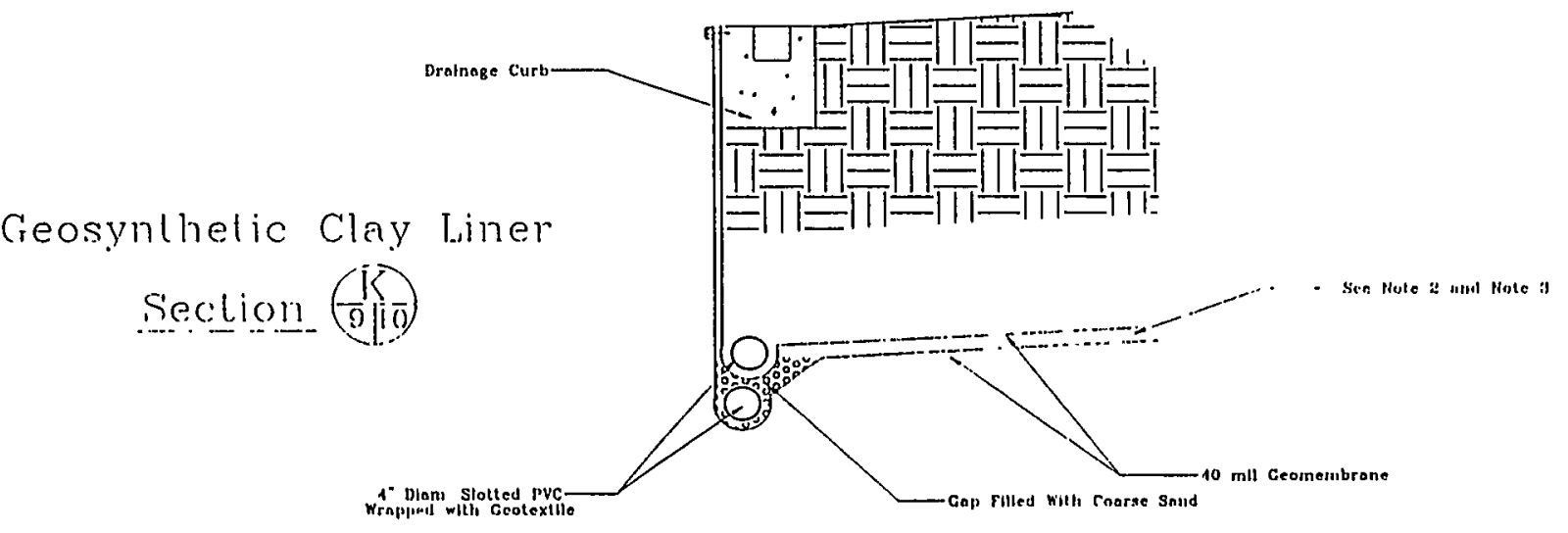

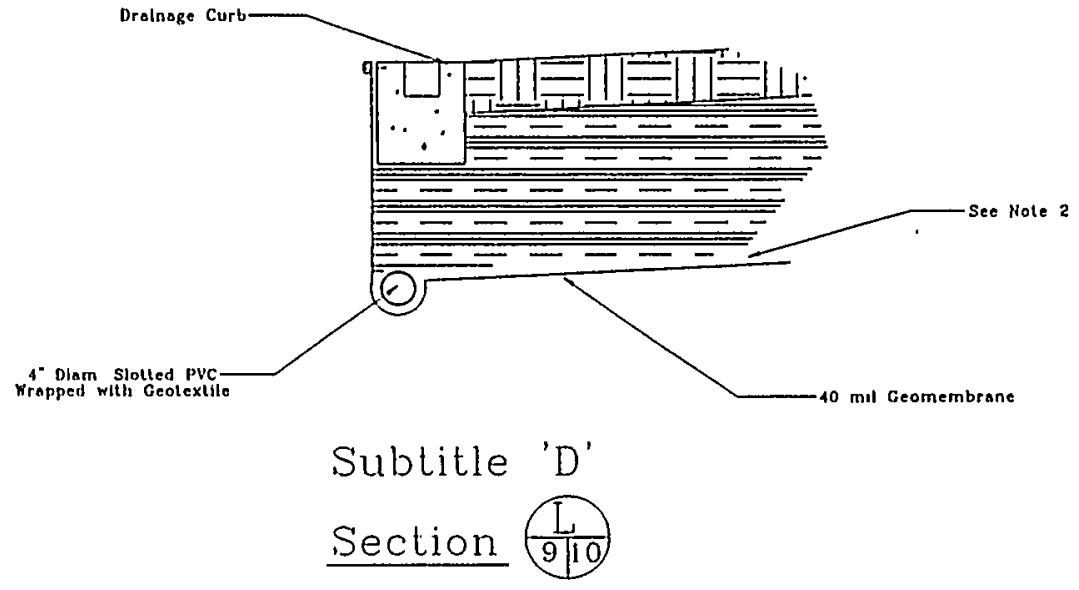

Noles:

Geotextile to be installed. Len out for clority 3) Gexnet to be installed. Lefl out for clarity Geosynthetic clay liner to be installed Let

Sandia National Laboratories office of T'echnology Development Environmental Restoration Technologies Mixed Waste Landfill Inlegrated Demonstralion Alternative landfill Cover Demonstrilion

profile or Rumoff C'ollection Pipes

\begin{tabular}{|c|c|}
\hline "1, & 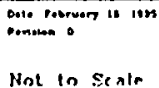 \\
\hline
\end{tabular}




\section{GUTT. DWG}
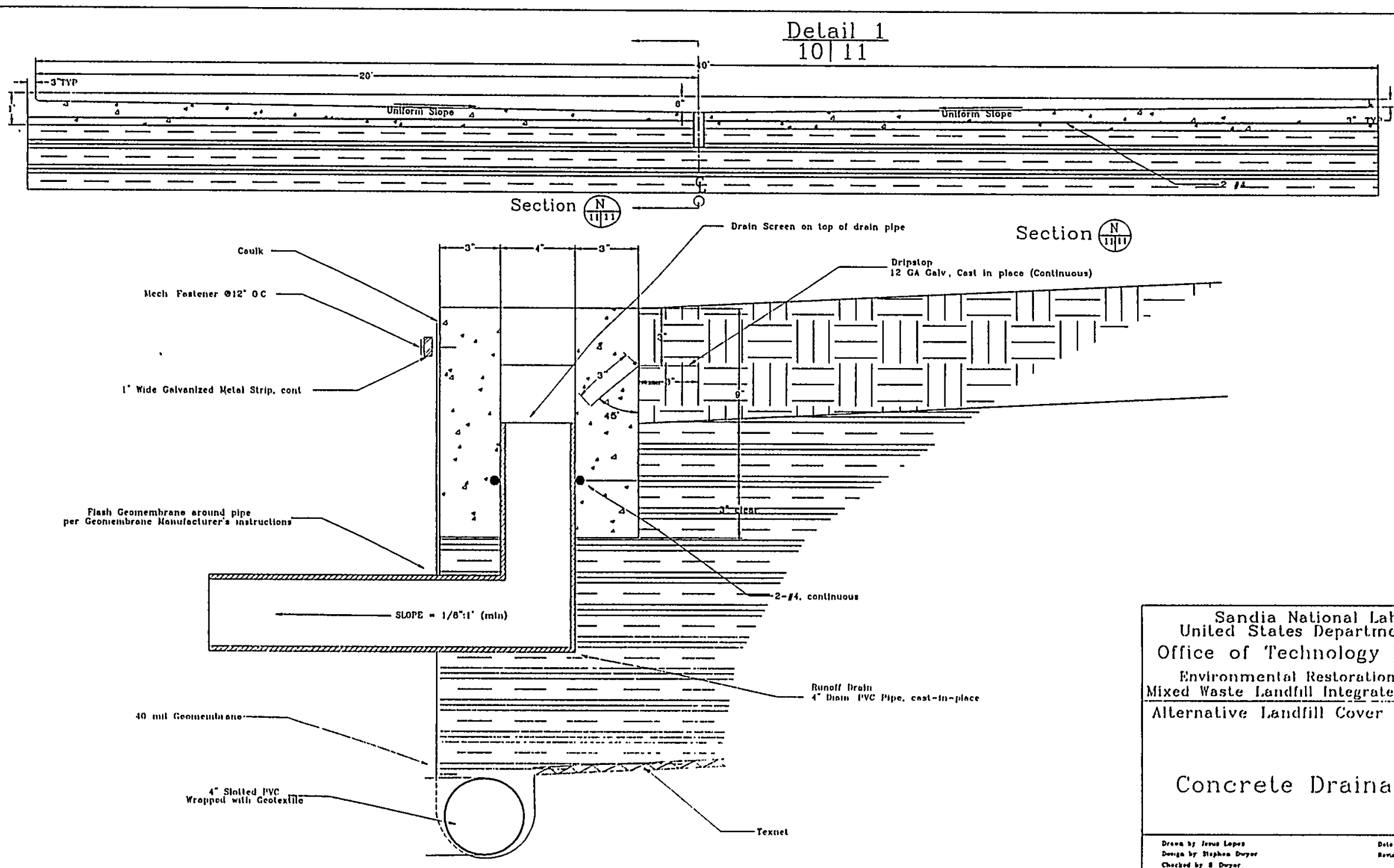

Section (1111)

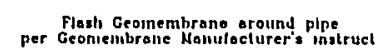

Sandia National Laboratories
United States Depart.ment of Office of Technology Development

Environmental Restoration Teschnologies Mixed Woste l.andfill integrated pernonsistration Alternative Landfill Cover Demonstmalion

Concrete Drainage Curb

\begin{tabular}{ll} 
& \\
\hline
\end{tabular}




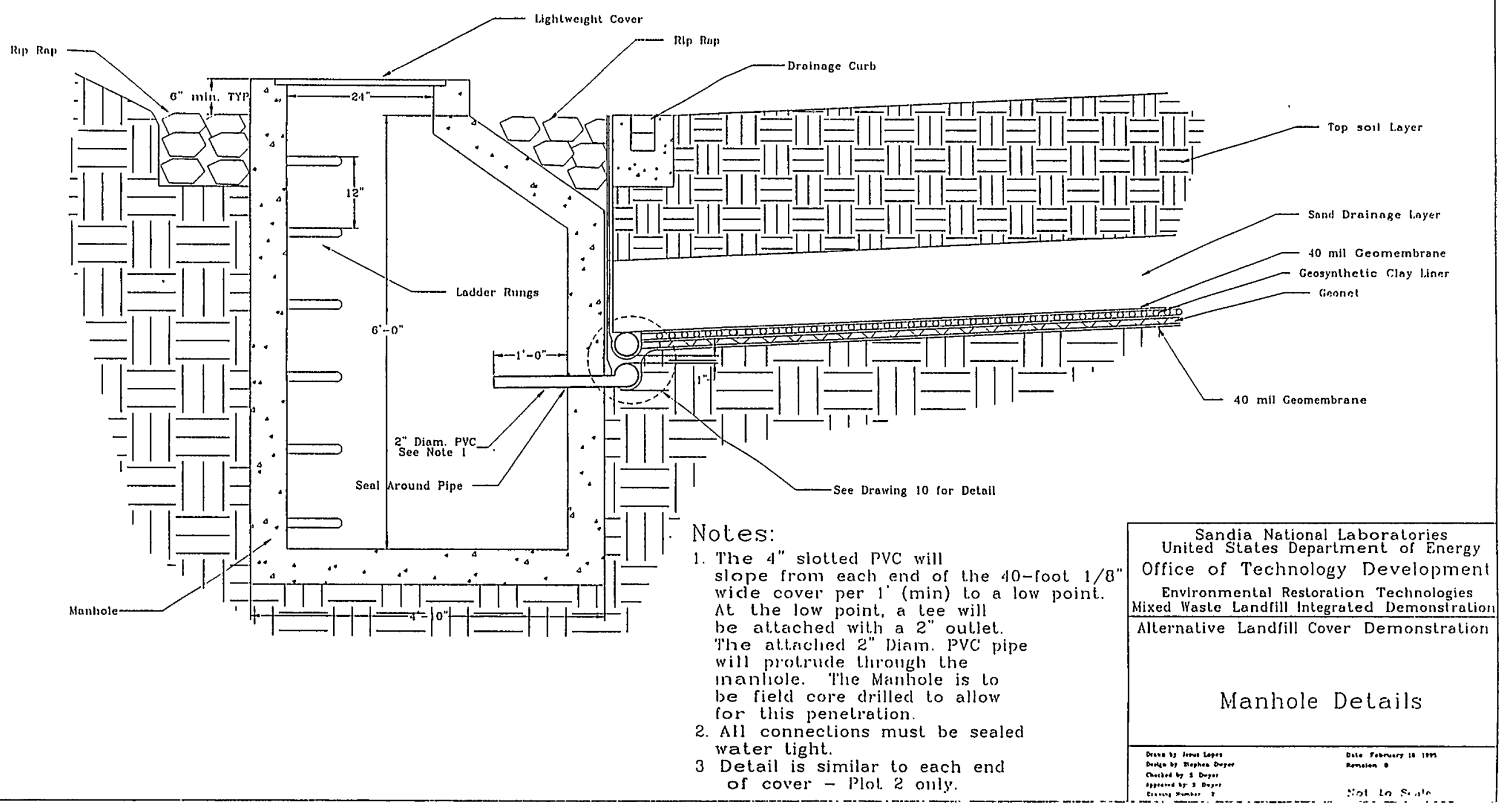




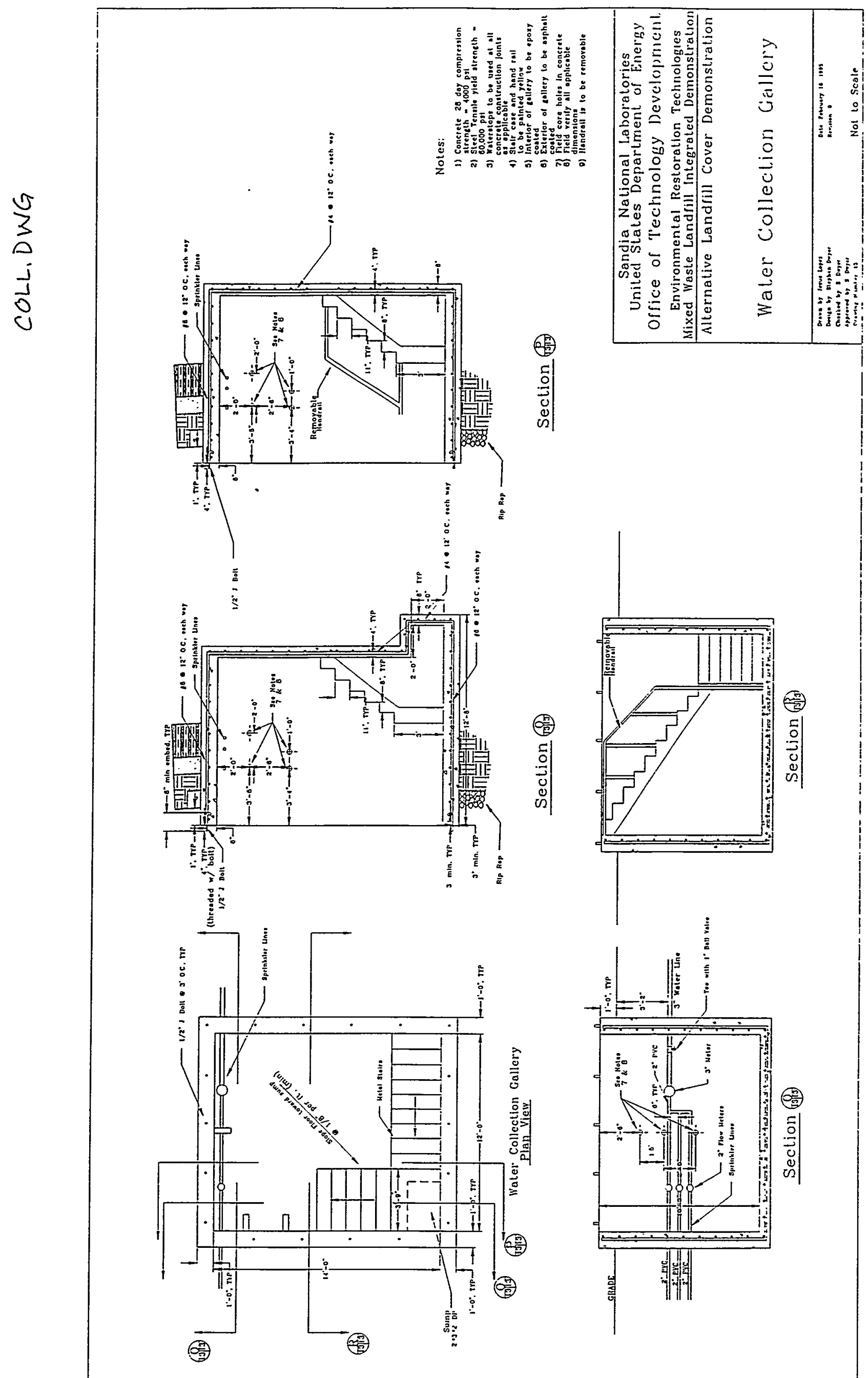




\section{RIPRAP. DWG}

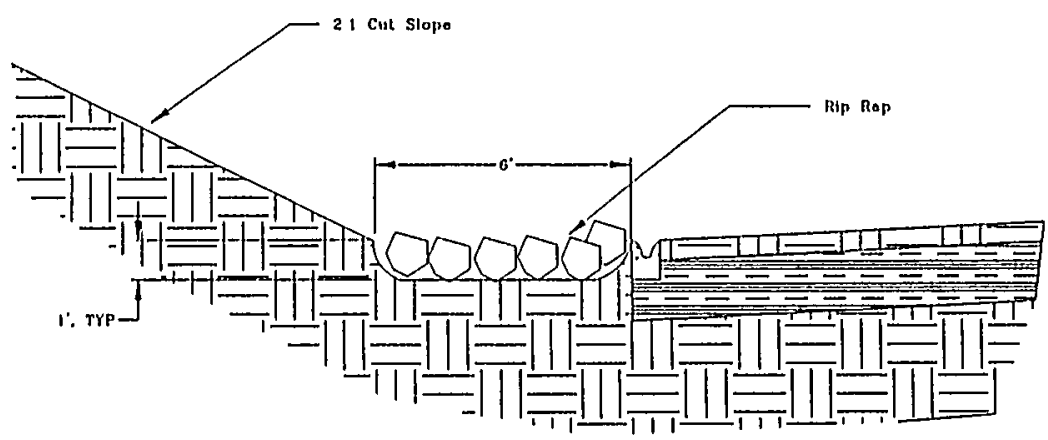

Section $\frac{1}{3 \cdot 8}$

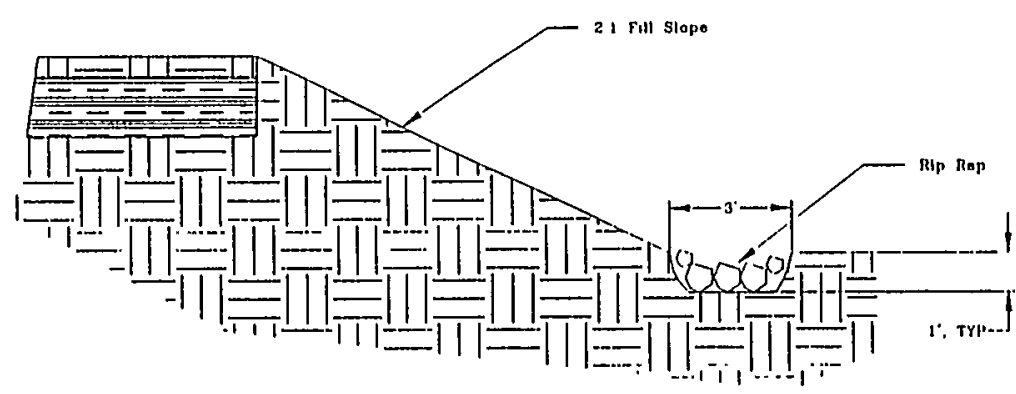

Section $\left(\frac{1}{3}\right)$

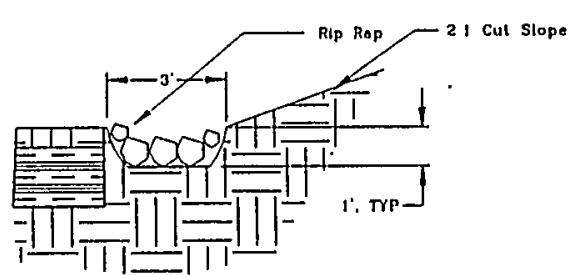

Section $\frac{K}{3 \sqrt{8}}$
Sandia National Laboralories
United Stales Department of Einergy office of Technology Development.

Environmental Resloration Pechlunologirs Mixed Waste Landfill Integrated Demoristration Alternalive Landfill Cover Demonstration

Rip Raps Dolails

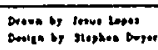

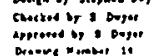

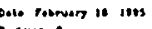

Nol 10 Senile 


\section{FDR, DWG}

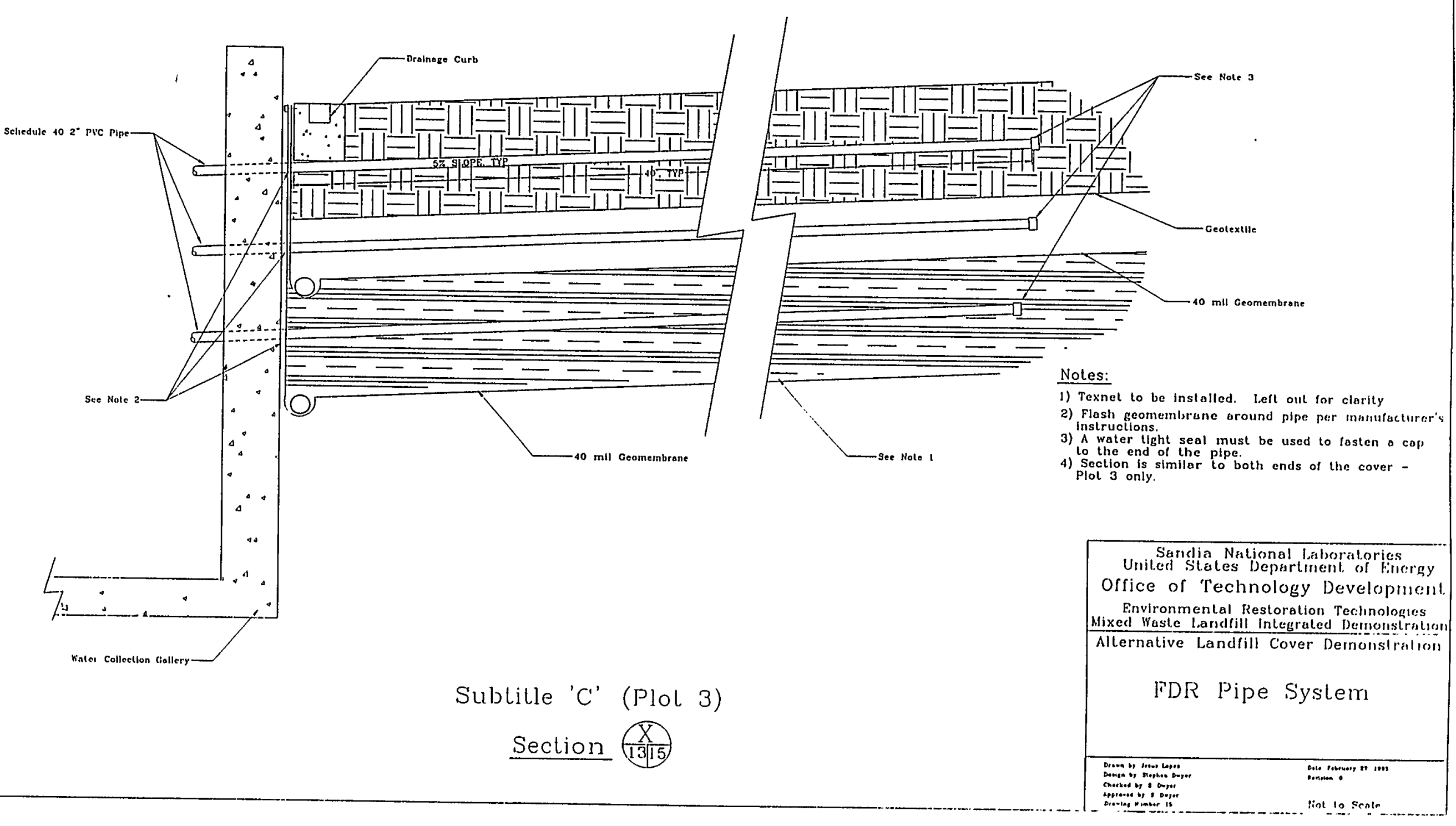




\section{ATTACHMENT C}

Phase I - Specifications 
Sandia National Laboratories

\title{
A7ternative Landfi11 Cover Demonstration
}

\author{
Specifications
}

$5-19-95$

prepared by:

Stephen F. Dwyer 


\section{SPECIFICATIONS}

\begin{tabular}{|c|c|}
\hline DIVISION 1 & GENERAL REQUIREMENTS \\
\hline SECTION 01000 & OVERVIEW OF TEST CONFIGURATION \\
\hline SECTION 01300 & DESCRIPTIVE SUBMITTALS \\
\hline DIVISION 2 & SITE WORK \\
\hline SECTION 02110 & CLEARING AND GRUBBING \\
\hline SECTION 02200 & EARTHWORK \\
\hline SECTION 02210 & RIPRAP \\
\hline SECTION 02725 & MANHOLES \\
\hline SECTION 02776 & GEOMEMBRANE \\
\hline SECTION 02777 & GEOSYNTHETIC CLAY LINER \\
\hline SECTION 02778 & GEOTEXTILES \\
\hline SECTION 02779 & DRAINAGE NETS \\
\hline SECTION 02780 & TEXNET \\
\hline SECTION 02900 & SEEDING \\
\hline SECTION 02901 & SPRINKLER SYSTEM \\
\hline DIVISION 3 & CONCRETE \\
\hline SECTION 03300 & CAST-IN-PLACE CONCRETE \\
\hline DIVISION 5 & METALS \\
\hline SECTION 05120 & STRUCTURAL STEEL \\
\hline SECTION 05500 & METAL FABRICATIONS \\
\hline DIVISION 9 & FINISHES \\
\hline
\end{tabular}




\section{SECTION 01000}

\section{OVERVIEW OF TEST CONFIGURATION}

Three covers will be installed side-by-side in Phase I. Phase I will be constructed in FY95. Phase II will be installed similarly continuing the side-by-side arrangement begun in Phase I. Phase II will be constructed in FY96. The plan dimensions of each cover will be 40 -feet wide by 300 -feet long. The 300 -foot length will be divided into two halves one sloping at $5 \%$ toward the east with the other half sloping at $5 \%$ toward the west. The north-south slope on each cover will be $0 \%$. The eastern half of each cover will have a sprinkler system installed in them to simulate various precipitation events during stress testing.

The covers were designed and will be constructed such that the cut volume of earth will approximately equal the fill required to construct the covers. Therefore, no borrow fill will be required. Refer to the drawings in Attachment A.

Plot 1 will be the EPA/RCRA Subtitle ' $D$ ' soil cover. These type of covers are generally used to close municipal landfills. This cover will be two-feet thick. The bottom 18inches will be compacted native soil. The earth will be placed such that it has a uniform minimum hydraulic conductivity of $1 \times 10^{-5} \mathrm{~cm} / \mathrm{sec}$. This layer is the barrier layer. Its primary purpose is to limit water infiltration through it. Subtitle ' $D$ ' regulations require this layer to be a minimum of 18 -inches thick with the lower of a (1) hydraulic conductivity less than or equal to the landfill liner or (2) $1 \times 10^{-5} \mathrm{~cm} / \mathrm{sec}$. The top sixinches of the cover will be topsoil. This layer is the vegetation layer and serves to establish native vegetation growth, minimize erosion, and protect the barrier layer. Subtitle ' $D$ ' regulations require a minimum of six-inches of earthen material for this layer. All layers will have a $5 \%$ slope.

Plot 2 will be identical to plot 3 with one exception. A GCL will be used in lieu of the two-foot thick compacted clay barrier layer. The GCL has a hydraulic conductivity of 5 $X 10^{-9} \mathrm{~cm} / \mathrm{sec}$. Therefore the entire section of plot 2 will only be about three-feet thick.

Plot 3 will be the EPA/RCRA Subtitle ' $C$ ' compacted clay cover. This cover will be approximately five-feet thick. The bottom two-feet will be the barrier layer. It will be compacted such that it has a minimum hydraulic conductivity of $1 \times 10^{-7} \mathrm{~cm} / \mathrm{sec}$. Because the soil at the site does not have adequate clay material in it, the soil will have to be amended prior to compaction by adding bentonite. Immediately above the clay barrier layer, a geomembrane will be placed to add a second barrier layer. The geomembrane will be placed in intimate contact with the soil barrier layer so they act as a composite barrier layer. Immediately above the geomembrane will be a one-foot thick layer of sand. This is a drainage layer designed to laterally route any water that has infiltrated the top vegetation layer quickly to collection drains located around the perimeter of the cover ( for this demonstration they will be located at the bottom of each slope). The drainage 
layer will not be compacted and will have a hydraulic conductivity of at least $1 \times 10^{-2}$ $\mathrm{cm} / \mathrm{sec}$. Above the drainage layer, a geotextile will be placed to act as a filter to keep the drainage layer material separate from the top vegetation layer soil. The top two-feet will be native soil. It will not be compacted. This layer will provide for vegetation growth, minimize erosion, and protect the barrier layer from harmful freeze/thaw action. This cover meets the minimum requirements for RCRA Subtitle ' $C$ ' covers. All layers will have a 5\% slope. Refer to the drawings in Attachment $\mathrm{A}$ for details of the above described test plots.

The plots will be instrumented to determine all variables in the water balance \{precipitation $(P)$, surface runoff $(R)$, lateral drainage $(D)$, soil water storage $(S)$, and percolation or infiltration (I)\}. equation with the exception of evapotranspiration (E) which will be estimated by solving the mass balance equation.

$$
\mathrm{E}=\mathrm{P}-\mathrm{I}-\mathrm{R}-\mathrm{D}-\Delta \mathrm{S} \quad \text { [Equation 1] }
$$

Precipitation and any water added to the covers with a sprinkler system will be quantified using a met station and/or flow meter on the sprinkler system. All water passing through the vegetation and barrier layers will be laterally routed to respective collection underdrain systems. Surface water runoff will be collected at the end of each respective slope with a gutter system. This collected water will then be routed through measurement devices to quantify it. TDRs will be strategically located throughout the covers at various depths to determine soil moisture contents that can in turn be interpolated to determine water storage capacities. Refer to section 8.0 of this Test Plan for descriptions of the instrumentation to be installed. The drawings in Attachment A show locations and details of the instrumentation. Sheds will be located on-site to house the data acquisition equipment to protect it from the weather. A met station will be located on-site to record all pertinent meteorological data.

The site has been designed so as to minimize runoff and run-on. Civil engineering cut/fill techniques were used to design the site. The cut taken from the site approximately equals the fill volume required to build the test plots. This ensures that soil specific to the sight is used for layers of the covers. Drainage is provided for around the perimeter of each cover.

A sprinkler system will be installed in the east half of each test plot. This system will be installed as designed in the drawings in Attachment A using the materials specified in Attachment B. Sandia's Facilities Engineering Division will provide rough-in of all utilities to the site including power and water. The water will be potable water. After all earthwork activities are complete including trenching, the test plots as well as all disturbed earthen areas will be seeded with native vegetation as specified. The seed will also be applied and fertilized as specified. 


\section{STANDARD SPECIFICATIION \\ SECTION 01300 \\ DESCRIPTIVE SUBMITTALS}

\section{PART 1 - GENERAL}

\subsection{DESCRIPTION OF WORK}

Section Includes: This section includes administrative and procedural requirements for submittals required for performance of the work, including:
A. Type A - Shop Drawings
B. Type B - Catalog Data
C. Type $\mathrm{C}$ - Installation Instructions
D. Type D - Operating Manual, Maintenance Instructions and Parts List
E. Type E-Internal Wiring Diagrams
F. Type S - Special

\subsection{PURPOSE OF SUBMITTAL REVIEW}

A. General Contractor Responsibility: The General Contractor's responsibility with respect to submittals shall be to review, stamp, and submit all submittals in the proper format and in a timely manner. The General Contractor shall be responsible for the following items and shall not submit a request for approval of said items but shall be ultimately and solely responsible for them:

1. Field verification and confirmation of all dimensions for proper fit of all materials. Sub-contractors' requests for field verification of dimensions shall be addressed by the General Contractor prior to submitting shop drawings to Sandia National Laboratories (the Sandia Delegated Representative).

2. Field verification and confirmation of quantities of materials.

3. Fabrication processes and techniques of construction.

B. Sandia Delegated Representative Responsibility: Submittals shall be reviewed by the Sandia Delegated Representative for the sole purpose of checking general conformance with the design concepts of the project and general compliance with the contract documents. 
Any action shown is subject to the requirements of the contract documents including plans and specifications and does not relieve the General Contractor from compliance with all provisions and requirements therein. Approval of a submittal does not relieve the General Contractor of his responsibility for correcting any errors which may exist in the submittal or for meeting the requirements of the contract documents.

\section{PART 2-MATERIALS}

\subsection{DESCRIPTIVE SUBMITTAL LISTS}

A. Submittal Codes: The following abbreviations: Arch., Civ., Struc., Mech., Elec., or Con. - identify the respective Architectural, Civil, Structural, Mechanical, Electrical or Controls discipline for which information is being requested.

B. Reference Numbers: Reference numbers are to be assigned to each submittal.

C. Submittal Types: The descriptive submittal types are used to identify the type of information being requested. They are defined below and are marked on the "Descriptive Submittals" review form.

1. Type A - Shop Drawings: Detailed drawings prepared specifically for the project and used for fabrication and/or assembly of material or equipment.

a. the Sandia Delegated Representative design/construction drawings shall not be reproduced and used for shop drawings.

b. Shop drawings shall be prepared accurately and to scale. Encircle deviations from the contract documents.

c. Shop drawings shall include dimensions, identification of products and materials, notation of special coordination requirements, notation of dimensions established by field measurements and shall indicate compliance with specified standards.

d. Shop drawings shall be submitted on sheets at least 8-1/2" $\times 11^{\prime \prime}(216$ $\mathrm{mm} \times 279 \mathrm{~mm}$ ) but no larger than 24" x 36" (610 mm x $914 \mathrm{~mm})$.

2. Type B - Catalog Data: Manufacturer's published literature describing the salient features of the product (e.g. size, weight, performance, code conformance, capacity, physical size, pictures, ratings, and construction features). Clearly highlight specified items and optional features such that there is no misunderstanding as to what precisely is being proposed.

3. Type C - Installation Instructions: Manufacturer's information explaining the preparation and installation of material and equipment, including: 
a. Outline drawings showing major components and dimensional service connection points.

b. Service connection requirements such as electrical fuse or circuit breaker size and type, pipe size and type, air duct, critical ambient conditions (cooling, heating), foundation requirements, and special precautions.

c. Adjustment requirements that must precede operation, including initial alignment procedures, leveling, purging, charging, and lubrication.

4. Type D - Operating Manual. Maintenance Instructions, and Parts List: Complete calibration procedures and operating instructions including all necessary pre-start up checks and safety precautions.

a. Provide instructions for step-by-step preventive maintenance and minor field repairs that will ensure optimum dependable performance, longevity, and life cycle cost.

b. Provide manufacturer's compiled parts list for maintenance and repair.

c. Provide a symptom, diagnostic, and corrective action schedule.

5. Type E - Internal Wiring Diagrams: Internal wiring and elementary (ladder) diagram for factory pre-wired equipment.

a. Include control diagrams that show the relative position of each component as a system diagram.

b. Include any additional information requested in the Mechanical and Electrical specifications for a particular product.

6. Type S - Special: Special information, samples and others as indicated.

a. Include actual color, texture, and pattern when submitting samples.

b. Indicate product sources and availability as well as manufacturer's name.

D. Asbestos Free and Lead Paint Free Certification Submittal: It is required that all products used on the construction project be "free of asbestos" and "free of lead paint." In addition to the above submittal types, for every product on which a submittal is requested, the Contractor shall also submit written certification from the product manufacturer stating that the products to be used on this project are "free of asbestos" and "free of lead paint."

\section{PART 3 - EXECUTION}

\subsection{SUBMITTAL PROCEDURES}


A. Coordination: Coordinate preparation and processing of submittals with performance of construction activities. Transmit each submittal in advance of performance of related construction activities.

1. Coordinate each submittal with fabrications, purchasing, testing, delivery, other submittals and related activities that require sequential activity.

2. Coordinate transmittal of different types of submittals for related materials so processing will not be delayed by the need to review related submittals concurrently.

The Sandia Delegated Representative reserves the right to withhold action on a submittal requiring review of related submittals until the related submittal information is received.

B. Review Time: The Sandia Delegated Representative may take two weeks for initial review. The Sandia Delegated Representative may also take additional time if processing must be delayed to permit review of related subsequent submittals. Allow additional time for processing of resubmittals.

C. Submittal Packaging: Bindable submittals, for each discipline, shall be three hole punched and bound into a card stock binder with flexible metal clips:

1. The submittal binder shall be stamped on the lower right hand corner of the cover with an approximately 4" x 4" (102 mm x $102 \mathrm{~mm})$ identification stamp as described below: .

"Discipline: " (e.g., Arch., etc.)

"Contract Number: " (e.g., TW-0030)

"Descriptive Submittal Reference Number: through 15 and 19 through 22)

"General Contractor Information:"

"Company Name: 11

"Company Phone Number: II

"General Contractor Reviewer Signature:

"General Contractor Reviewer Printed Name: (e.g., $\quad 1$ 2. The General Contractor shall submit descriptive submittals to the Sandia

- General Contractor's Reviewer shall sign his name on the identification stamp on the cover of the binder indicating General Contractor's approval of the submittal and acknowledgment as to the appropriateness of the material or information submitted for use in construction. 
Unstamped, unsigned, or otherwise incomplete submittals shall not be accepted by the Sandia Delegated Representative.

3. Submittals shall be grouped together with related products of the same discipline when possible (e.g., Finishes - vinyl floor tile, floor tile adhesive, vinyl base, ceiling tile, ceiling tile grid, carpet tile, carpet tile adhesive, ceramic tile, ceramic tile adhesive). The requested submittal types for a particular item should always be complete and submitted together. Do not submit one type of submittal apart from other types of submittals for the same product. Each submittal shall be legible and grouped together in the following order for each product requested:

a. Asbestos and Lead Paint Free Certification(s)

b. Submittal Type A - Shop Drawings. Fold shop drawings down to 8$1 / 2^{\prime \prime} \times 11^{\prime \prime}(216 \mathrm{~mm} \times 279 \mathrm{~mm})$ and insert into submittal package.

c. Suḅmittal Type B - Catalog Data

d. Submittal Type C - Installation Instructions

e. Submittal Type D - Operating Manual, Maintenance Instructions and Parts List

f. Submittal Type E - Internal Wiring Diagrams

g. Submittal Type S - Special: (Only bindable special submittals including color charts and fabric swatch charts.) Unbindable special submittals shall be submitted under separate cover and per product specification.

D. SUBMITTAL DELIVERY: For all types of submittals except samples, transmit the number of submittals needed for the General Contractor's purposes plus two (2) copies to be retained by the Sandia Delegated Representative. Submittals received from sources other than the General Contractor shall not be accepted. Incomplete, improperly packaged and/or submittals which have not been stamped by the General Contractor shall be returned without action. When submitting samples, submit two (2) sets of samples for review unless otherwise requested.

1. Deliver descriptive submittals to the Sandia Delegated Representative within 15 calendar days after the Notice to Proceed, except: 1) as may be stipulated elsewhere in this Contract, or 2) for "original drawing(s)" prepared by the General Contractor for specialty systems, such as: HVAC Controls, fire protection sprinklers and/or lightning protection system, which shall be delivered within 30 calendar days after the Notice to Proceed.

2. Material and/or equipment shall not be installed until requested submittals pertaining to said material and/or equipment have been approved. 
E. SUBMITTAL REVIEW: Submittals will be reviewed and stamped with a self-explanatory action stamp. The stamp will be appropriately marked, as follows, to indicate the action taken:

1. Final Unrestricted Release: When submittals are marked "Approved", that part of the work covered by the submittal may proceed provided it complies with requirements of the contract documents; final acceptance will depend upon that compliance. The term "Approved" shall only indicate that there are no exceptions taken to the submittal.

2. Final But Restricted Release: When submittals are marked "Approved Except as Noted," that part of the work covered by the submittal may proceed provided it complies with notations or corrections on the submittal and requirements of the contract documents; final acceptance will depend upon that compliance.

3. Returned for Resubmittal: When submittal is marked "Not Approved, Revise and Resubmit", do not proceed with that part of the work covered by the or prepare a new submittal in accordance with the notations and resubmit without delay. Repeat as necessary to obtain a different action mark.

\section{PART 4 - ITEMS TO BE SUBMITTED}

\subsection{ITEMS TO BE SUBMITTED}

The following items are to be submitted as a minimum:

\begin{tabular}{|l|c|c|c|c|c|l|}
\hline \multicolumn{1}{|c|}{ Type } & A & B & C & D & E & \multicolumn{1}{|c|}{ S } \\
\hline Item & $\bullet$ & & & & & \\
\hline Stairs & $\bullet$ & $\bullet$ & & $\bullet$ & & \\
\hline Sprinkler System & & $\bullet$ & $\bullet$ & & & Mixing method \\
\hline Bentonite & & $\bullet$ & $\bullet$ & & & \\
\hline Seed & & $\bullet$ & $\bullet$ & & & \\
\hline Fertilizer & & $\bullet$ & $\bullet$ & & & $\begin{array}{l}\text { Including } \\
\text { tackifier }\end{array}$ \\
\hline Reinforced. Concrete & $\bullet$ & $\bullet$ & & & & $\begin{array}{l}\text { Conc. \& Reinf. } \\
\text { Steel }\end{array}$ \\
\hline Geomembrane & $\bullet$ & $\bullet$ & $\bullet$ & & & $\begin{array}{l}\text { Installation } \\
\text { Layout }\end{array}$ \\
\hline Geosynthetic Clay Liner & $\bullet$ & $\bullet$ & $\bullet$ & & & $\begin{array}{l}\text { Installation } \\
\text { Layout }\end{array}$ \\
\hline $\begin{array}{l}\text { TexNet (Drainage net \& } \\
\text { Geotextile) }\end{array}$ & $\bullet$ & $\bullet$ & $\bullet$ & & & $\begin{array}{l}\text { Installation } \\
\text { Layout }\end{array}$ \\
\hline Geotextile & $\bullet$ & $\bullet$ & $\bullet$ & & & $\begin{array}{l}\text { Installation } \\
\text { Layout }\end{array}$ \\
\hline
\end{tabular}




\begin{tabular}{|l|c|c|c|c|c|l|}
\hline Sand & & $\cdot$ & & & & $\begin{array}{l}\text { Sieve Analysis, } \\
\text { Hydraulic } \\
\text { Conductivity } \\
\text { Test Results }\end{array}$ \\
\hline Riprap & & $\bullet$ & & & & \\
\hline Slotted PVC Pipe & & $\bullet$ & & & & \\
\hline PVC Pipe & & $\bullet$ & & & & \\
\hline Adhesives & & $\bullet$ & & & & \\
\hline Paint & & $\bullet$ & $\bullet$ & & & \\
\hline Manholes & & $\bullet$ & $\bullet$ & & & \\
\hline Equipment & & & & & & $\begin{array}{l}\text { All equip. to be } \\
\text { used, where, \& } \\
\text { how. }\end{array}$ \\
\hline Epoxy Coating & & $\bullet$ & $\bullet$ & & & \\
\hline Anchors & $\bullet$ & $\bullet$ & & & & \\
\hline Ashphaltic Coating & & $\bullet$ & $\bullet$ & & & \\
\hline
\end{tabular}

END OF SECTION 01300 


\section{SECTION 02110}

\section{CLEARING AND GRUBBING}

\section{DESCRIPTION}

1 This work consists of clearing, grubbing, removing, and disposing of vegetation and debris within the limits of the right of way, easement areas borrow pits, and other areas shown in the Contract or required by the work. Vegetation and objects designated to remain shall be preserved free from injury or defacement.

\section{CONSTRUCTION REQUIREMENTS}

2 The SNLA Delegated Representative will designate all trees shrubs, plants, and other objects to remain. Any object that is designated to remain and is damaged shall be repaired or replaced as directed by the Engineer, at the Contractor's expense. Clearing and grubbing shall extend to the toe of fill or the top of cut slopes, unless otherwise designated. All surface objects, trees, stumps, roots, and other protruding obstructions not designated to remain shall be cleared and grubbed, including mowing, as required. Undisturbed stumps, roots, and nonperishable solid objects located two feet or more below subgrade or embankment slope may remain in place. In areas to be rounded at the tops of backslopes stumps shall be removed to at least two feet below the surface of the final slope line.

Except in areas to be excavated, all holes resulting from the removal of obstructions shall be backfilled with suitable material and compacted in accordance with section 02200 .

Burning of perishable material will not be permitted. They must be hauled off and disposed of in an acceptable landfill.

No material or debris shall be disposed of within the project limits without the written permission of the SNLA Delegated Representative. Material or debris that is disposed of within the project limits shall be buried to a depth of at least one foot and the surface shall be reshaped to match the adjacent ground line. The Contractor shall make all arrangements to obtain written permission from property owners for disposal locations outside the limits and view of the project. Copies of this written agreement shall be furnished to the SNLA Delegated Representative before the disposal area is used.

All cleared merchantable timber shall be removed from the project and shall become the property of the Contractor. 
The Contractor shall scalp the areas within the excavation or embankment grading limits. Mowed sod need not be removed where the embankment to be constructed is four feet or more in height. Scalping shall include the removal from the ground surface of brush, roots, sod, grass, residue of agricultural crops, sawdust, and other vegetable matter.

END OF SECTION 02110 


\section{SECTION 02200}

\section{EARTHWORK}

\section{PART 1 - GENERAL}

\section{$1.1 \mathrm{SCOPE}$}

A. This Specification Section describes the requirements for the following:

1. The earthwork related to the following operations:

a. Dust control.

b. Excavation of materials from the site and construction of the cover system.

c. Construction of the final cover systems.

d. Subgrade preparation for the covers and permanent drainage ditches.

e. Finish grading of the site.

2. Furnishing and installing displacement monuments as shown on the Contract Drawings.

\subsection{RELATED WORK}

A. Section 01300 Descriptive Submittals

B. Section 02110 Clearing and Grubbing

C. Section 02900 Seeding.

\subsection{DEFINITIONS}

A. Excavation: Excavation is defined as excavation required to reach the lines and grades indicated on the Contract Drawings or specified herein. It shall include excavation of topsoil, silt, clay, sand, gravel, talus, soft or disintegrated rock, boulders or detached pieces of solid rock.

B. Contaminated Materials Excavation: There are no contaminated materials at this site.

C. Unicontaminated Materials Excavation: Excavation of uncontaminated materials including topsoil, silt, clay, sand, gravel, talus, soft or disintegrated rock, boulders 
and removal of detached pieces of solid rock carried out to reach lines and grades indicated on the Contract Drawings or specified herein. This excavation shall include excavations for cover, drainage ditches, and finish grading.

D. Overexcavation: Overexcavation is defined as excavation carried out beyond the lines and grades indicated on the Contract Drawings or in the Contract Specifications.

E. Percent Maximum Density: Percent maximum density is a percentage of the maximum density obtained by the test procedure presented in ASTM D698, as' applicable.

F. Topsoil: Topsoil is the existing surface soil stripped to a depth of six-inches and consisting of natural, friable soil representative of productive soils in the vicinity. Topsoil shall be free of any admixture of subsoil, foreign matter, objects larger than two-inches in any dimension, toxic substances, and any material or substance that may be harmful to plant growth.

G. Subgrade Preparation: Subgrade preparation includes fine grading and compaction of excavations for the landfill cover systems including drainage ditches, backfills, and cover upon which bedding materials, riprap, or other features are to be constructed.

H. Cover: Cover shall consist of multiple layers of varied materials placed to form test plots as shown on the Contract Drawings.

\subsection{APPLICABLE PUBLICATIONS}

A. The Publications listed below form a part of this Specification to the extent referenced. The Publications are referred to in the text by the basic designation only:

1. American Society for Testing and Materials (ASTM):

C33-85 Specifications for Concrete Aggregates

D422-63 Method for Particle-Size Analysis of Soils

D698-91 Test methods for Moisture-Density Relations of Soils and SoilAggregate Mixtures Using $5.5 \mathrm{lb}$. (2.49-kg) Rammer and 12-in. (305-mm) Drop

D1140-92 Test Method for Amount of Material in Soils Finer than the No. 200 (75-um) Sieve 
D1556-90 Test method for Density of Soil in Place by the Sand-Cone Method

D2216-80 Test Method for Laboratory Determination of Water (Moisture) Content of Soil, Rock, and Soil-Aggregate Mixtures

D2487-93 Test Method for Classification of Soils for Engineering Purposes

D2922-91 Test Methods for Density of Soil and Soil-Aggregate in Place by Nuclear methods (Shallow Depth)

D4318-93 Test Method for Liquid Limit, Plastic Limit, and Plasticity Index of Soils

\subsection{QUALITY ASSURANCE}

A. The Contractor is responsible for meeting all requirements set forth in the contract documents. The SNLA Delegated Representative will take soil samples and perform moisture-density, gradation and other tests to ascertain that the work is being performed in compliance with these Specifications. Samples may be taken at the place of excavation, stockpiles, or on the fill itself. The SNLA Delegated Representative will conduct the density and other tests on the fill and related laboratory testing as frequently as the SNLA Delegated Representative considers necessary. The Contractor shall remove surface material and render assistance as necessary to enable sampling and testing.

B. Methods of Sampling and Testing:

1. In-Place Density: ASTM D1556 or D2922

2. Liquid Limit, Plastic Limit and Plasticity Index: ASTM D4318

3. Particle Size Analysis: ASTM D422

4. Percentage Passing No. 200 Sieve: ASTM D1140

5. Moisture Content: ASTM D2216, D2922

6. Laboratory Moisture-Density Relations: ASTM D698

7. Soil Classification: ASTM D2487

C. Suitability of Materials: The suitability of all materials for foundations and backfill will be approved by the SNLA Delegated Representative . Fill material 
will be approved material from off-site borrow areas and / or required excavations.

D. The SNLA Delegated Representative may direct that inspection trenches or test pits be cut into fills to determine that the Specifications have been met. Such trenches or pits will be of limited depth and size, and shall be backfilled with the material excavated therefrom, or other fill material meeting the requirements for the zones cut into. Backfill shall be compacted to a density at least equal to that of the contiguous fill .

E. Inspection trenches or test pits, and the refilling of the same, shall be at the Contractor's expense.

\section{PART 2 - PRODUCTS}

\subsection{FILL AND INFILTRATION MATERIALS}

A. General:

1. Fill materials shall be obtained from cut excavations. Sand and bentonite shall be purchased off-site and hauled on-site.

2. The Contractor shall make his own determination of any processing that may be required, and shall perform testing as required to meet the Specifications for the various construction materials.

3. The SNLA Delegated Representative shall be granted access to each proposed source to collect samples for testing. The SNLA Delegated Representative may perform additional tests to determine if the materials meet the requirements specified herein.

4. Approval will be based on evidence of compliance with the requirements specified herein.

B. Gradations: Gradations specified shall be as determined after delivery to the site or after cut has been made and material is ready to be refilled.

C. Fill and Infiltration Materials:

1. General: Fill materials for general fill shall conform to the following requirements:

a . All materials except peat (PT), organic clay or organic silt (OL or $\mathrm{OH})$ as classified by ASTM D2487 and other materials free from roots, organic 
matter, trash, debris, frozen materials, and stones generally not larger than two-inches in diameter.

b. Fill material, except for demolition debris and except for clearing and stripping debris, shall not contain more than one percent organic material, by weight, or other deleterious substances.

2. Infiltration Barrier Materials: Infiltration barrier materials shall conform to the following requirements.

a. Infiltration barrier fill materials shall be uncontaminated soils obtained from the cut excavation.

b. Infiltration barrier material shall not contain clearly visible organic matter or other-deleterious substances.

c. Soil requirements:

Plot 1: Infiltration barrier materials shall consist predominantly of soils with classification of $\mathrm{SC}, \mathrm{SM}, \mathrm{CL}, \mathrm{CH}, \mathrm{MH}$ when classified in accordance with the requirements of ASTM D2487, and graded with maximum particle size of two-inches. The Laboratory hydraulic conductivity of these materials when compacted to $95 \%$ of maximum dry density per ASTM D698 (verified by SNL per ASTM D2922 and ASTM D1556) shall be equal to or below $1 \times 10^{-5} \mathrm{~cm} / \mathrm{sec}$ (verified by SNL per ASTM D5093). Compliance with these Specifications will be determined by the SNLA Delegated Representative.

A 24-foot by 24-foot test plot of this infiltration barrier layer is to also be constructed near the plot 1 cover. This test plot will be flat with no slope. All other specifications followed for the cover will be adhered to with the test plot. Immediatley after completetion of the construction of this test plot, it is to be covered with a clean sheet of plastic. The sheet of plastic is to be secured against wind uplift (e.g. with sand bags).

Plot 3: Infiltration barrier materials shall consist predominantly of soils with classification of SC, SM, CL, CH, MH when classified in accordance with the requirements of ASTM D2487, and graded with maximum particle size of two-inches and minimum of $20 \%$ percent passing No. 200 Sieve. The barrier material will be limited to a maximum of $10 \%$ gravel passing No. 4 sieve per ASTM D422. Such materials shall have a Plasticity Index (PI) between 10 to $35 \%$ when tested according to ASTM D4318. The Laboratory hydraulic conductivity of these materials when compacted to $98 \%$ of maximum dry density per ASTM D698 (verified by SNL per ASTM D2922 and ASTM D1556) shall be equal to or below $1 \times 10^{-7} \mathrm{~cm} / \mathrm{sec}$ (verified by SNL per ASTM D5093). Bentonite must be 
added to the fill material to achieve the desired properties. The bentonite to be added must be a minimum of $6 \%$ by weight. The soil mixture. is to be placed at $4 \%+/-2 \%$ wet of optimum. Compliance with these Specifications will be determined by the SNLA Delegated Representative.

A 24-foot by 24-foot test plot of this infiltration barrier layer is to also be constructed near the plot 3 cover. This test plot will be flat with no slope. All other specifications followed for the cover will be adhered to with the test plot. Immediatley after completetion of the construction of this test plot, it is to be covered with a clean sheet of plastic. The sheet of plastic is to be secured against wind uplift (e.g. with sand bags).

3. Bentonite: (for plot 3) Used to help reduce permeability in infiltration barrier layer.

a. Bentonite shall be untreated Sodium bentonite, suitable for use in a variety of liquid or effluent conditions, "Envirogel-10", as manufactured by WyoBen, Inc., Billings, Montana, or approved equal.

b. Bentonite shall meet the requirements of specification $13 \mathrm{~A}$ of the American Petroleum Institute.

4. Sand - Drainage Layer:

a. Clean, coarse sand with a general particle size distribution of $2 \mathrm{~mm}$ to $0.25 \mathrm{~mm}$ (verified by SNL per ASTM D1140 and ASTM D422). No more than $5 \%$ will be allowed to pass through the no. 200 sieve. Special care must be taken when placing sand so as not to damage liner or disturb the barrier layer. The hydraulic conductivity of the drainage layer must be greater than or equal to $1 \times 10^{-2} \mathrm{~cm} / \mathrm{sec}$ (test results verifying compliance with this to be supplied by contractor to SNL - also to be field verified by SNL). The General Contractor must provide a submittal showing that the sand to be used meets the hydraulic conductivity requirement.

\subsection{EQUTPMENT}

All equipment to be used on the project must be submitted to and approved by the SNLA Designated Representative.

A. The mixer for mixing bentonite with the cover material shall be capable of thoroughly mixing and controlling the percentage, by weight, of bentonite, soil, and water. The mixer shall be one of the following, or approved equal:

1. Self-propelled road mixer, Seaman TO-730H. 
2. Portable pug-mill.

3. Portable drum roll asphalt plant, Cedarapids 4820 (with continuous flow process ).

B. Compaction equipment:

1. Kneading -- Compactor weight in the range of 30,000 to $40,000 \mathrm{lbs}$. (feet must be substantially less than the loose lift thickness so as not to damage any instrumentation placed in prior lift).

2. Smooth Roll -- Compactor weight in the range of 30,000 to $40,000 \mathrm{lbs}$..

\section{PART 3 - EXECUTION}

\subsection{DUST CONTROL}

A. Dust control shall consist of furnishing water supply, required equipment, additives, accessories and incidentals, and applying water during the completion of the Contract, as required by the SNLA Delegated Representative . A minimum moisture content of four percent shall be maintained in the upper 6-inches of the roadbeds at all times.

B. Water shall be applied by means of pressure-type distributors or pipe lines equipped with a spray system or hoses with nozzles that will insure a uniform application of water.

C. All equipment used for the application of water shall be equipped with a positive means of shut-off.

D. Unless otherwise permitted by the SNLA Delegated Representative or unless all the water is applied by means of pipe lines, at least one mobile unit with a minimum capacity of 5,000 gallons shall be available for applying water on the project at all times.

E. If the Contractor uses chemical additives in water, furnishing and applying the additives shall be at the Contractor's expense.

F. The use, location of application, and the amount and type of additives proposed for use by the Contractor must be submitted to and approved by the SNLA Delegated Representative prior to their use.

\subsection{EXCAVATION}


A. Preparation:

1. Required lines, levels, contours and datum shall be identified before the start of excavation.

B. General:

1. At all times during excavation, the Contractor shall conduct his operations in such a manner as to prevent free standing water and contamination of uncontaminated materials. The Contractor shall, as a minimum, maintain positive grade to safeguard against such problems.

2. The Contractor shall perform required excavation to the lines and grades indicated on the Contract Drawings. He shall remove all excavated material from the excavation site and dispose of it in the designated spoil areas or use it for other purposes, as approved.

3. Unsuitable or low density subgrade material not readily capable of in-place compaction shall be excavated as directed by the SNLA delegated representative and disposed of as a topsoil.

4. Adequate working space for safety of personnel shall be provided within the limits of the excavation.

5. All unstable material, large stones, debris and incompressible soils shall be removed from bottoms of the excavation to a minimum depth of 12-inches.

6. Except as otherwise noted, care shall be exercised to preserve the material below and beyond the lines of all excavation. Where excavation is carried below grade, the Contractor shall backfill to the required grade or to indicated invert grade, as specified, and recompact the backfill to meet the existing conditions.

7. Excavation for the convenience of the Contractor shall conform to the limits approved by the SNLA Delegated Representative and shall be at no additional expense to SNLA.

8. Where practicable, suitable materials removed from excavation shall be used as fill or backfill.

C. Materials Excavation:

1. General: Materials excavation shall include excavations of materials from the various areas of the site. The excavated materials shall be used as fill in various areas of the site including the construction of berms, dikes, general 
fill, roadway fill, structure fill, backfill, and fill for the final grading of the . site, as required. Excavated material may be stockpiled for later use.

2. Drainage Swales Excavation:

a. General: Swales shall be cut accurately to the cross sections and grades where indicated. All roots, stumps, rock, and foreign matter in the sides and bottom of swales shall be trimmed and dressed or removed to conform to the slope, grade, and shape of section indicated. Care shall be taken not to excavate ditches below the grades indicated. Excessive ditch excavation shall be backfilled to grade with satisfactory, thoroughly compacted material. Ditches shall be maintained until final acceptance of the work. Except the north and east swale excavation may be extended to provide adequate fill for the cover construction.

b. Permanent Drainage Swales:

Ditches shall be excavated true to line and grade. Any erosion which occurs to ditch excavation before placing erosion protection materials shall be repaired with compacted backfill. All such repairs shall be at Contractor's expense.

The top six-inches of the subgrade shall be compacted as specified in Article 3.4 below. After compaction has been completed, finish grading shall be done in such a manner that the sideslopes are rendered smooth surfaces. All rocks, brush, roots, large clods, and other objects shall be removed.

c. The area to be excavated must be cleared and grubbed in accordance with section 02110. All topsoil will be stockpiled for later use.

D. Embankment Materials:

1. Materials excavated from the site, including excavations for trenches, and drainage ditches shall be used as fill for construction of various features including site grading, or stockpiled for later use, or wasted in the spoil area, as specified in this Section and is required by the SNLA Delegated Representative.

2. Where used in fills, such material shall be transported directly from the excavation and placed in its final position in such fills whenever possible. If required by the Contractor's schedule, the material may be placed temporarily in stockpiles at approved locations. Material in stockpile shall be protected from contamination of any kind that would render it unsuitable for use in fills. 
E. Unsatisfactory or Excess Materials: Unsatisfactory and excess. excavated uncontaminated material generated during the Work and not approved for use in the Work shall be disposed of in the designated stockpile/spoil areas as directed by the SNLA Delegated Representative.

F. Garbage, refuse, debris, oil, and any waste material which is generated by the Contractor and is harmful to the environment or offensive to the area shall be removed from the job site and disposed of offsite in a manner approved by the authority having jurisdiction over the disposal site.

G. All operations in the stockpile/spoil areas throughout the Work shall be in strict conformity with the requirements of this Section.

\subsection{FILL CONSTRUCTION}

A. General Requirements:

1. Fill materials shall be placed and compacted to the lines and grades shown on the Contract Drawings .

2. If any portion of the materials does not meet the specified requirements, the Contractor shall remove such material and replace it with fill materials meeting the specification at no additional cost.

B. Placing Requirements:

1. Prepared subgrade: Prior to placement of Materials, the in-place density of the top six-inches of the area receiving the fill shall be at least 95 percent of maximum dry density as determined by ASTM D698. If necessary, the surface shall be plowed, borrowed materials mixed, materials added or removed and compacted to obtain the specified densities.

2. Fill materials may require moisture conditioning (wetting or drying) prior to compaction.

3. Fill materials shall be placed in continuous and approximately horizontal layers for their full length and width unless otherwise specified.

4. The method of dumping and spreading the materials shall ensure uniform distribution of the material.

5. The loose thickness of each layer of materials shall not be greater than that required to achieve the required compaction, and in no case shall exceed 12inches for the fill and nine-inches for the infiltration barrier materials. These thicknesses are dependent on the compaction equipment to be used. The 
kneading foot length must be substantially less than the loose lift thickness so as not to damage any instrumentation placed in prior lift.

6. The Contractor shall place material to a grade of one percent or steeper to preclude the ponding of water. No fill shall be placed on any area where ponding has been allowed to occur. Where ponding has occurred and the fill material is required to be placed, the water shall be removed and the subgrade reprocessed to 95 percent of ASTM D698.

7. Materials shall not be placed on frozen subgrade or embankment material foundations, nor shall frozen material be used as fill.

C. Compaction / Remolding Requirements:

1. Each layer of shaping fill materials leading to the final prepared subgrade shall be compacted to a minimum of 95 percent of maximum dry density as determined by ASTM D698. The infiltration barrier for plot 1 will be compacted to a minimum of 95 percent of maximum dry density as determined by ASTM D698. The infiltration barrier for plot 3 will be compacted to a minimum of 98 percent of maximum dry density as determined by ASTM D698.

2. During compaction the moisture content of shaping fill material shall be maintained to achieve the specified density. Uniform moisture distribution shall be obtained by disking, blading, or other methods approved by the SNLA Delegated Representative prior to compaction of a layer. During compaction of infiltration barrier materials for plots 1 and 3, moisture content shall be maintained at $4 \%+1-2 \%$ wet of optimum moisture content as determined by ASTM D698.

3. All lift surfaces shall be scarified and moistened by sprinkling to an acceptable moisture content prior to placement of the next layer of fill with the exception of the final lift of the barrier layer in plot 3. That surface shall be smoothrolled. No object larger than $1 / 2$-inch shall be visible on this surface. This surface must be smooth with a uniform slope to allow for complete contact of the geomembrane when installed on top.

4. If the rolled surface of any layer of the fill in place is too wet for proper compaction of the layer of fill material to be placed thereon, it shall be removed, allowed to dry or worked with harrow, scarifier, or other suitable equipment to reduce the water content to the required amount, and then recompacted before the next succeeding layer of fill is placed.

5. Fill placed at densities lower than the specified minimum density or at moisture contents outside the specified acceptable range of moisture content 
shall be reworked to meet the density and moisture requirements or removed and replaced by acceptable fill compacted to meet these requirements.

D. Field Quality Control:

1. General: The SNLA Delegated Representative will take samples and perform tests throughout the construction period, and the Contractor shall cooperate in providing access for the SNLA Delegated Representative to areas where testing is to be performed and shall schedule his placing to avoid interference with the testing operations.

2. Tests: The contractor will be responsible for performing his own tests to ensure compliance with all contract provisions. Copies of these tests are to provided to the Sandia Delegated Representative. The SNLA Delegated Representative will also perform tests. The Contractor must make allowances for these tests at no additional cost to Sandia. Refer to the ALCD QA plan for approximate number and type of tests to be performed by SNLA personnel.

\subsection{Subgrade Preparation For Permanent Drainage Ditches.}

The top six-inches of the subgrade of each permanent drainage ditch shall be compacted to a minimum of 95 percent of maximum density as determined by ASTM D698. After compaction has been completed, fine finishing shall be done in such a manner that the side-slopes are smooth surfaces. All rocks, brush, roots, large clods, and other objects shall be removed before placement of the bedding materials.

3.5 Subgrade Preparation of Infiltration Barrier for Placement of Geomembrane.

Surface shall be smooth rolled. No rocks, clods, or other form of debri shall be exposed larger than one-half inch in diameter.

END OF SECTION 02200 


\section{SECTION 02210}

\section{$\underline{\text { RIPRAP }}$}

\section{PART 1 - GENERAL}

\subsection{SCOPE}

This Specification Section describes the requirements for furnishing and placing rip rap and bedding materials for the cover and permanent drainage ditches.

\subsection{RELATED WORK}

A. Section 01300 Descriptive Submittals.

B. Section 02200 Earthwork: Subgrade preparations.

\subsection{APPLICABLE PUBLICATIONS}

A. The publications listed below form a part of this Specification to the extent referenced. The publications are referred to in the text by the basic designation only:

1. American Society for Testing and Materials (ASTM):

C88-83 Test Method for Soundness of Aggregates by Use of Sodium Sulfate or Magnesium Sulfate

C127-84 Test Method for Specific Gravity and Absorption of Coarse Aggregate

C131-81 Resistance to Degradation of Small-Size Coarse Aggregate by Abrasion and Impact in the Los Angeles Machine

C295-85 Practice for Petrographic Examination of Aggregates for concrete

C535-81 Test Method for Resistance to Degradation of Large-Size Coarse Aggregate by Abrasion and Impact in the Los Angeles Machine

PART 2 - Products

\subsection{MATERIALS}


A. Material Sources: Erosion protection materials shall be obtained from sources approved by the SNLA Delegated Representative. Every change in source of materials shall require approval from the SNLA Delegated Representative.

1. Approval of the source as a borrow area does not mean that all materials excavated will meet the requirements of this Specification. Processing or selective quarrying may be necessary to meet the quality requirements of this Section.

2. The basis for approval of the sources proposed by the Contractor shall be a site inspection report by an engineering geologist or geotechnical engineer which will include, as a minimum, an evaluation of soundness, hardness, and durability for three samples representative of the proposed source.

B. All materials shall be free from radioactive or other contamination.

C. Rip rap materials:

1. Individual pieces shall be dense, sound, resistant to abrasion, and shall be free from cracks, seams, and other defects as shown in the petrographic examination.

2. The shape of at least seventy percent of the material, by weight, shall be such that the minimum dimension is not less than one third of the maximum dimension. No crushed stones may be used because the sharp edges may damage the liners. Preferably rounded to surrounded stones will be used.

3. Quality:

Tests

Specific Gravity

(Saturated Surface

Dry Basis)

Absorption

Soundness

Abrasion
Designation

ASTM C127

Not more than 2.45

Requirements

ASTM C127

ASTM C88

ASTM C131
Not more than 1.5 percent.

$\mathrm{NaSO}_{4}$ Test: Not more than 5 percent loss of weight after 5 cycles.

Not more than 35 percent (Los Angeles Machine) loss of weight after 500 revolutions. Ratio of weight 
loss after 100 revolutions to loss after 500 revolutions shall not exceed 30 percent.

Abrasion

Petrographic
ASTM C535

ASTM C295
Not more than 50 percent (Los Angeles Machine) loss of weight after 1000 revolutions. Ratio of weight loss after 200 revolutions to loss after 1000 revolutions shall not exceed 20 percent.

The Contractor shall furnish a report for review by the Engineer.

4. The riprap shall be sized as follows: maximum size stone - 8-inches, median size stone - 5-inches, minimum layer thickness - 12-inches. The size of an individual stone shall be determined by measuring its long dimension.

Gradation is to be well graded with at least $50 \%$ of the gradation between the maximum size stone and the median size stone. The remaining riprap materials shall be reasonably well graded within the following limits:

\begin{tabular}{cc}
$\begin{array}{l}\text { U.S. Standard Sieve Size } \\
\text { (Square Openings) } \\
\text { Material Size }\end{array}$ & $\begin{array}{c}\text { Percent Passing } \\
\text { (by weight) }\end{array}$ \\
\hline $4 "$ & 100 \\
2" & $30-95$ \\
$1 "$ & $20-50$ \\
$\# 4$ & $0-20$ \\
100 & $0-5$
\end{tabular}

\section{PART 3 - EXECUTION}

\subsection{PLACEMENT AND COMPACTION}

A. Subgrade preparation (for drainage ditches, etc.) shall conform to Specification Section 02200.

B. Where the required layer thickness is 12 -inches or less, the material shall be spread in one layer.

C. The riprap will not require compaction. 
D. Riprap material shall be placed so that the larger pieces are uniformly distributed and the smaller pieces serve to fill the spaces between them to provide wellkeyed, densely placed layers of riprap of the specified thicknesses.

E. Riprap material may be placed by end-dumping and may be spread by bulldozers or other suitable equipment. The material shall not be dropped from a height exceeding two-feet above the riprap material, nor in a manner to cause fracture, displacement, or damage to the material being placed or already in place. In no case shall the riprap be dropped directly on the liners but shall be dumped on already placed riprap and then pushed into its final position .

F. Construction equipment other than spreading and placement equipment shall not be allowed to move over the placed riprap material layers.

\subsection{TOLERANCES}

A. The material layers shall be placed to the limits and thicknesses shown on the Contract Drawings within the following tolerances:

1. Top of bedding material shall be within 0.1 foot of elevations shown on the Contract Drawings.

2. The minimum in-place thickness shall not be more than 100 percent of the thickness shown.

3. The maximum in-place thickness shall not be more than 135 percent of the thickness shown.

4. Local irregularities will be permitted provided that such irregularities do not form noticeable mounds, ridges, swales, or depressions.

\subsection{FIELD QUALITY CONTROL}

A. The placement of the materials shall be monitored to ensure that the following requirements are met:

1. The correct type of material is being placed.

2. The material being placed is clean and free of unsuitable material.

3. The material is being loaded, transported, and placed in a manner which minimizes segregation. 
4. Deviations from minimum and maximum thickness shall not exceed 50 percent of the maximum rock size.

5. The material is being placed to line and grade within the tolerances and limits designated in Article 3.2 above.

B. All unsatisfactory materials shall be removed to the satisfaction of the SNLA Designated Representative and replaced with acceptable materials at no additional cost.

C. The SNLA Designated Representative may require that material which has become segregated or not placed according to the above requirements to be regraded or adjusted, using appropriate equipment, to conform with the tolerances and limits given above, at no additional cost.

\section{END OF SECTION 02210}




\section{SECTION 02725}

\section{MANHOLES}

\section{PART 1 - GENERAL}

\subsection{DESCRIPTION OF WORK}

A. This specification shall apply to the materials and operations required for the installation of manholes.

B. The extent of the work is indicated on the Contract Drawings.

1.02 RELATED WORK

1. Section 01300 "Descriptive Submittals".

2. Section 02200 "Earthwork".

3. Section 03300 "Concrete".

4. Section 09900 "Painting".

\subsection{QUALITY ASSURANCE}

A. The materials and practices comprising the work shall conform to this and other referenced standard specifications.

\section{PART 2 - PRODUCTS}

\subsection{MATERIALS}

A. Cement shall conform to the following requirements:

1. Cement for use in mortar shall conform to ASTM C 91.

2. Portland cement for use in precast and cast-in-place concrete, and in masonry grout and mortar shall conform to ASTM C 150, type II, IIA, or III.

B. Block, Brick, Precast Barrels, Steps, Frames and Covers:

1. Riser Sections and Tops: Precast reinforced concrete conforming to ASTM C 478. Precast tops shall be either eccentric cone or flat slab type. Concrete 
for precast sections shall have a minimum of 4000 psi 28 -day compressive strength.

2. Riser Joints: Rubber gasket conforming to ASTM C 443, bituminous mastic gasket, or butyl rubber gasket.

3. Concrete Masonry Units: Solid precast segmental block conforming to ASTM C 139.

4. Mortar: Type M.

5. Brick: Grade MS, conforming to ASTM C 32.

6. Steps: Steel reinforced polypropylene conforming to ASTM C 478.

7. Frames and Covers: Lightweight - capable of supporting a 300-pound vertical load.. Bearing surfaces shall be ground or machined, such that the cover shall seat firmly onto the frame without rocking. Submit for approval.

\section{PART 3 - EXECUTION}

\subsection{MANHOLE CONSTRUCTION}

A. General: Manholes shall be installed at the locations and elevations indicated and as detailed on the Contract Drawings. Installed pipe invert elevations at manholes shall be as designated on the Contract Drawings.

B. Manhole Bases: Prior to manhole installations, prepare a subgrade soil foundation a minimum of 12 " beneath the elevation of the bottom of the concrete manhole base and compact to $95 \%$ maximum density as determined by ASTM D698. Cast-in-place concrete for the base shall have a minimum of 3000 psi 28- day compressive strength.

C. Joints in precast riser sections and tops shall be made using gasket materials in accordance with the manufacturer's written installation instructions.

D. Precast Concrete Manhole Wall Sections:

1. Wall sections are to be precast concrete only.

2. Holes for pipe sections can be precast at designated locations. However, the preferred method is to core drill the pipe holes on-sight to allow for possible field variations in pipe locations. 
E. Pipe sections built into manhole walls shall be reinforced with grout so that the manhole and adjoining pipe sections are monolithic.

F. Manhole Floors: Manhole floors shall be made of grout and the work shall be free of any rough corners or sudden changes in direction such that a steady uniform flow with a minimum of wave action will be provided. Changes in direction and grade will consist of the largest curve radius the manhole diameter will permit. Free vertical drop from any branch or service line shall not exceed one half the mainline pipe diameter measured from the mainline upstream invert.

G. Manhole steps shall be cast-in-place or driven into precast or site-drilled holes. Steps shall be installed not more than 12 " apart vertically on the interior wall directly beneath the manhole cover according to ASTM C 478.

H. Manhole frames and covers shall be installed as detailed and adjusted to required elevation by building up courses of manhole brick on full beds of mortar with "push joints" or by using precast concrete adjustment rings set in full beds of mortar.

I. Fill all lifting holes and other voids inside and outside with non-shrink grout. The inside of the manhole shall be cleaned of all loose mortar, framing materials and other debris.

J. Bituminous waterproof coating shall be applied to the exterior surface of the manhole. When designated on the Contract Drawings, the interior of the manhole shall be coated with a corrosion resistant material.

END OF SECTION 02725 


\section{SECTION 02776}

\section{GEOMEMBRANE}

1.0 Related Work.

A. Section 01300 Descriptive Submittals.

B. Section 02200 Earthwork: Subgrade Preparation.

\subsection{Material}

2.1 The geomembrane is to be a 40 mil linear low density polyethylene membrane meeting the following properties:

\begin{tabular}{|c|c|c|c|c|}
\hline $\begin{array}{l}\text { Resin Properties } \\
\text { Melt Flow Index } \\
\text { Resin Density }\end{array}$ & $\begin{array}{c}\text { Method } \\
\text { ASTM D } 1238\end{array}$ & $\begin{array}{l}\text { Units } \\
\mathrm{g} / 10 \mathrm{~min} \\
\mathrm{~g} / \mathrm{cm}^{3}\end{array}$ & $\begin{array}{c}\text { Minimum } \\
1.3 \\
0.925\end{array}$ & $\begin{array}{c}\text { Typical } \\
0.94 \\
0.918\end{array}$ \\
\hline $\begin{array}{l}\text { Sheet Properties } \\
\text { Thickness }\end{array}$ & $\begin{array}{l}\text { Method } \\
\text { ASTM D 751, } \\
\text { NSF mod. }\end{array}$ & Units & Minimum & Typical \\
\hline Average & & mils & 40.0 & 41.5 \\
\hline Individual & & mils & 38.0 & 40.3 \\
\hline Density & ASTM D 1505 & $\mathrm{~g} / \mathrm{cm}^{3}$ & 0.938 & 0.929 \\
\hline Carbon Black Content & $\begin{array}{l}\text { ASTM D } 3015 \\
\text { NSF mod. }\end{array}$ & rating & $\mathrm{A} 1, \mathrm{~A} 2, \mathrm{~B} 1$ & $\mathrm{~A} 1$ \\
\hline Tensile Properties & ASTM D 638 & & & \\
\hline Stress at Yield & & $\begin{array}{l}\text { psi } \\
\text { ppi }\end{array}$ & $\begin{array}{c}1640 \\
66\end{array}$ & $\begin{array}{c}1720 \\
71\end{array}$ \\
\hline Stress at Break & & $\begin{array}{l}\text { psi } \\
\text { ppi }\end{array}$ & $\begin{array}{c}3800 \\
152\end{array}$ & $\begin{array}{c}4600 \\
191\end{array}$ \\
\hline Strain at Yield & $\begin{array}{l}1.3 " \text { gage length } \\
\text { (NSF) }\end{array}$ & percent & 15.0 & 20.0 \\
\hline \multirow[t]{2}{*}{ Strain at Break } & $\begin{array}{l}2.0 " \text { gage or } \\
\text { extensometer }\end{array}$ & percent & 750 & 880 \\
\hline & $\begin{array}{l}\text { 2.5" gage length } \\
\text { (NSF) }\end{array}$ & percent & 600 & 700 \\
\hline Tear Resistance & ASTM D 1004 & $\begin{array}{l}\text { ppi } \\
\text { lbs. }\end{array}$ & $\begin{array}{c}590 \\
24\end{array}$ & $\begin{array}{l}625 \\
26\end{array}$ \\
\hline \multirow[t]{2}{*}{ Puncture Resistance } & $\begin{array}{l}\text { FTMS 101C, } \\
\text { Method 2065 }\end{array}$ & ppi & 1450 & 1500 \\
\hline & & lbs & 58 & 62 \\
\hline
\end{tabular}


2.2 Material Delivery .

The SNLA Delegated Representative will be present, whenever possible, to observe and assist in material delivery and unloading on site. The SNLA Delegated Representative is to note any materials received in a damaged state and note any material damaged during unloading. Upon mobilization to site, a SNLA Delegated Representative shall:

- Verify the equipment used on site is adequate and does not risk damage to the geomembrane or other materials.

- Mark rolls or portions of rolls which appear damaged.

- Verify that storage of materials ensures adequate protection against dirt, theft, vandalism, and passage of vehicles.

- Ensure that rolls are properly labeled and that labeling corresponds with Quality Control documentation and Shipping Bills of Lading.

- Assist SNLA Delegated Representative, if present, with material sampling or record keeping.

- Complete roll numbers, date, bill of lading number, roll size, and any damage noted will be logged on the Material Delivery Checklist.

\section{GEOMEMBRANE INSTALLATION}

\subsection{Earthwork}

3.1.1 The General and/or Earthwork Contractor shall be responsible for preparing and maintaining the subgrade in a condition suitable for installation of the liner unless specifically agreed otherwise. Refer to section 02200 .

\subsubsection{The following general minimum guidelines shall be followed.}

3.1.2.1 Surfaces to be lined shall be smooth and free of debris, roots, and angular or sharp rocks. All fill shall consist of well-graded material, free of organics, trash, clay balls, or other deleterious material that may cause damage to the geomembrane. The upper six inches of the finished subgrade shall not contain stones or debris larger than onehalf inch. The subgrade shall be compacted in accordance with design specifications, but in no event less than is required to provide a firm, unyielding foundation sufficient to permit the movement of vehicles and welding equipment over the subgrade without causing rutting or other deleterious effects. The subgrade shall have no sudden sharp or abrupt changes in grade.

3.1.2.2 The Earthwork Contractor shall protect the subgrade from -desiccation, flooding, and freezing. Protection, if required, may 
consist of a thin plastic protective cover (or other material as approved by the engineer) installed over the completed subgrade until such time as the placement of geomembrane liner begins. Subgrades found to have desiccation cracks greater than one-half inch in width or depth, or which exhibit swelling, heaving, or other similar conditions shall be replaced or reworked by the General and/or Earthwork Contractor to remove these defects.

3.1.2.3 Surface Acceptance. Upon request, the manufacturer's representative will provide the Owner's and/or Contractor's Representatives with a written acceptance of the surface to be lined. This acceptance will be limited to the amount of area that the contractor is capable of lining during a particular work shift. Direction and control of any subsequent repairs to the subgrade, including the subgrade surface, shall remain the responsibility of the Earthwork Contractor.

\subsection{Preparation for Geomembrane Deployment}

\subsubsection{Panel Layout}

Prior to commencement of liner deployment, layout drawings shall be produced to indicate the panel configuration and general location of field-seams for the project. The layout must be submitted to and approved by the SNLA Delegated Representative.

\subsubsection{Identification}

Each panel used for the installation shall be given a numeric or alpha-numeric identifier. This panel identification number shall be related on the Panel Placement Form to a manufacturing roll number that identifies the resin type, batch number, and date of manufacture.

\subsection{Field Panel Placement}

\subsubsection{Weather Conditions}

Geomembrane deployment will generally not be done during any precipitation, in the presence of excessive moisture (i.e. fog, dew), in an area of standing water, or during high winds.

\subsubsection{Location}

The Contractor is to install field panels as indicated on the approved layout drawing. If the panels are deployed in a location other than that indicated on the approved layout drawings they will be removed and replaced in correct 
locations. If any materials are damaged during this process, they will be replaced at the contractor's expense. The revised location shall be noted in the field on a record "as-built" drawing which will be modified at the completion of the project to reflect actual panel locations. Record drawings will be maintained and submitted by the Contractor.

\subsubsection{Documentation of Panel Placement}

Information relating to geomembrane panel placement including date, time, panel number, and panel dimensions will be maintained on the Panel Placement Form.

3.3.3.1 If a portion of a roll is set aside to be used at another time, the roll number will be written on the remainder of the roll in several places.

\subsubsection{Method of Deployment}

3.3.4.1 The method and equipment used to deploy the panels must not damage the geomembrane or the supporting subgrade surface.

3.3.4.2 No personnel working on the geomembrane will wear shoes that can damage the geomembrane or engage in actions which could result in damage to the geomembrane.

3.3.4.3 Adequate temporary loading and/or anchoring, (i.e. sandbags, tires), which will not damage the geomembrane, will be placed to prevent uplift of the geomembrane by wind.

3.3.4.4 The geomembrane will be deployed in a manner to minimize wrinkles.

3.3.5 Any area of a panel seriously damaged (torn, twisted, or crimped) will be marked and repaired in accordance with Paragraph 5.3 of this document.

\subsection{Geomembrane Field Seaming}

\subsubsection{General Requirements}

\subsubsection{Layout}

In general, seams shall be oriented parallel to the slope, i.e., oriented along, not across the slope. Whenever possible, horizontal seams should be located on the base of the cell, not less than five feet from the toe of the slope. Each seam made in the field shall be numbered and indicated on the record drawings: Seaming information to include seam number, 
welder ID, machine number, temperature setting, and weather conditions will be maintained on a Panel Seaming Form.

\subsubsection{Personnel}

All personnel performing seaming operations shall be trained in the operation of the specific seaming equipment being used and will qualify by successfully welding a test seam as described in Paragraph 3.5.3. The project foreman will provide direct supervision of the seaming operations.

\subsubsection{Equipment}

\subsection{Fusion Welding}

Fusion Welding consists of placing a heated wedge, mounted on a self propelled vehicular unit, between two overlapped sheets such that the surface of both sheets are heated above the polyethylene's melting point. After being heated by the wedge, the overlapped panels pass through a set of pre-set pressure wheels which compress the two panels together to form the weld. The fusion welder is equipped with a device which continuously monitors the temperature of the wedge.

\subsection{Extrusion Fillet Welding}

Extrusion fillet welding consists of introducing a ribbon of molten resin along the edge of the overlap of the two geomembrane sheets to be welded. A hot-air preheat and the addition of molten polymer causes some of the material of each sheet to be liquefied resulting in a homogeneous bond between the molten weld bead and the surfaces of the overlapped sheets. The extrusion welder is equipped with gauges giving the temperature in the apparatus and a numerical setting for the pre-heating unit.

\subsubsection{Weather Conditions}

Many factors, such as the geomembrane temperature, humidity, wind, precipitation, etc. , can effect the integrity of field seams and must be taken into account when deciding whether or not seaming should proceed. Test seams, as described in Paragraph 3.5.3, are required prior to daily production seaming to determine if the weather conditions will effect the Contractor's ability to produce quality seams. Additional non- 
destructive and destructive testing of production seams substantiate the decision made by the Project Superintendent to seam on any given day.

\subsubsection{Seam Preparation}

\subsubsection{Fusion Welding}

3.4.2.1.1 Overlap the panels of Geomembrane approximately four to six inches prior to welding.

3.4.2.1.2 Clean the seam area prior to seaming to assure the area is clean and free of moisture, dust, dirt or debris of any kind. No grinding is required for fusion welding.

3.4.2.1.3 Adjust the panels so that seams are aligned with the fewest possible number of wrinkles and "fish mouths".

3.4.2.1.4 A movable protective layer may be used, at the discretion of the Contractor, directly below the overlap of geomembrane that is to be seamed to prevent build-up of dirt or moisture between the panels.

\subsubsection{Extrusion Fillet Welding}

3.4.2.2.1 Whenever possible, extrusion welded seams will be prebeveled prior to heat-tacking into place.

3.4.2.2.2 Overlap the panels of geomembrane a minimum of three inches.

3.4.2.2.3 Using a hot-air device, temporarily bond the panels of geomembrane to be welded, taking care not to damage the geomembrane.

3.4.2.2.4 Clean the seam area prior to seaming to assure the area is clean and free of moisture, dust, dirt, and debris of any kind.

3.4.2.2.5 Grind seam overlap prior to welding within one hour of the welding operation in a manner that does not damage the geomembrane. Grind marks should be covered with extrudate whenever possible. In all cases grinding should not extend more than one-quarter inch past the edge of the area covered by the extrudate during welding. 
3.4.2.2.6 Purge the extruder prior to beginning the seam to remove all heat-degraded extrudate from the barrel. The purged extrudate will be placed on scrap material so as to prevent contact with installed geomembrane.

\subsection{Keep welding rod clean and dry.}

\subsubsection{Trial Welds}

Trial welds shall be conducted by Welding Technicians prior to each seaming period, every five hours, as weather conditions dictate, or as requested by the SNLA Delegated Representative if welding problems are suspected. All trial welds will be conducted under the same conditions as will be encountered during actual seaming. Once qualified by a passing trial weld, welding technicians will not change parameters (temperature, speed, etc.) without performing another trial weld.

\subsubsection{Trial Weld Length}

The trial weld shall be made by joining two pieces of geomembrane at least six inches in width. Trial welds for fusion welds will be 15 feet long and extrusion weld trial seams will be a minimum of four feet long.

\subsubsection{Sample Procedure}

3.4.3.2.1 Visually inspect the seam for squeeze out, footprint, pressure, and general appearance.

3.4.3.2.2 Cut three one-inch wide specimens, one from the middle of the seam and one-foot from each end of the test seam. Specimens shall be obtained using a one-inch die cutter. The specimens shall then be tested in peel using a field tensiometer.

3.4.3.2.3 In order for a trial weld to be considered acceptable, all three specimens must meet the following criteria:

1. Exhibit Film Tearing Bond (FTB).

2. Meet or exceed the manufacturer's minimum peel strength values. If any specimens are in nonconformance, the entire procedure shall be repeated. In the case of double track fusion welded seams, both welds must pass in order to be considered acceptable. 
3.4.3.2.4 If repeat tests utilizing reasonable sets of welding parameters also fail, the seaming apparatus shall not be accepted and shall not be used for seaming until the deficiencies are corrected and a passing test seam is achieved.

\subsubsection{Trial Weld Documentation}

3.4.3.3.1 The SNLA Delegated Representative will be present during peel testing. The contractor will record date, time, operator, machine number, ambient and operating temperatures, speed setting, peel values, and pass/fail designation.

3.4.3.3.2 All trial weld records shall be maintained and submitted on a trial Weld Form to the SNLA Delegated Representative.

3.4.3.3.3 The SNLA Delegated Representative will give final approval to proceed with welding after observing trial welds.

\subsubsection{General Seaming Procedures}

3.4.4.1 Seaming shall extend to the outside edge of panels to be placed in the anchor trench.

3.4.4.2 While welding a seam, monitor and maintain the proper overlap.

3.4.4.3 Inspect seam area to assure it is clean and free of moisture, dust, dirt and debris of any kind.

3.4.4.4 Welding technicians will periodically check machine operating temperature and speed, and will mark this information on the geomembrane.

3.4.4.5 Align wrinkles at the seam overlap to allow welding through the wrinkle.

3.4.4.6 "Fish mouths" or wrinkles at seam overlaps, which cannot be welded through, shall be cut along the ridge in order to achieve a flat overlap. The cut "fish mouth" or wrinkle shall be heat-tacked flat and extruded or patched with an oval or round patch of the same geomembrane extending a minimum of three inches beyond the cut in all directions.

3.4.4.7 All cross/butt seams between two rows of seamed panels shall be welded during the coolest time of the day to allow for contraction of the geomembrane. 
3.4.4.8 Prior to welding cross/butt seams, the top and bottom overlap of intersecting fusion welded seams will be trimmed six inches. Intersecting extrusion fillet welded seams will be ground to flatten the extrusion bead prior to welding butt seams.

3.4.4.9 All "T" joints produced as a result of cross/butt seams shall be extrusion fillet welded. The overlap on each "leg" of the "T" joint will be trimmed back six inches. Then grind three inches minimum on each of the three legs of the "T" and extrusion weld all of the area prepared by grinding.

3.4.4.10 Whenever possible, welding technicians will cut a one-inch peel specimen at the end of every seam. Prior to welding the next seam, the specimen will be tested for peel. The SNLA Delegated Representative may request additional trial welds, based on observations of peel test specimens.

3.4.4.11 In the event non-complying seam test strips are encountered, the welding machine will be taken out of service until a passing trial weld is obtained, and additional peel specimens will be taken to localize the flaw.

3.4.4.12 Results of field seam test strips will be maintained and submitted to the SNLA Delegated Representative.

3.4.4.13 The SNLA Delegated Representative may, after consulting with the Contractor, take destructive samples from any seam, if defects are suspected.

\subsubsection{Seaming Documentation}

3.4.5.1 All seaming operations will be documented by the Contractor and submitted to the SNLA Delegated Representative. Welding technicians will mark on the liner with Mean Streak permanent markers at the start of all seams information regarding date, time, Welding Technician ID, machine number, and machine operating temperature and speed. The Contractor will record date, time, seam number, Technician ID, machine ID, set temperature, speed, and weather condition on a Panel Seaming Form and submit it to the SNLA Delegated Representative.

3.4.5.2 Welding technicians will periodically check operating temperature and speed and mark the information along the seam.

3.4.5.3 The SNLA Delegated Representative will make periodic checks on welding operations to verify overlap, cleanliness, etc. 


\section{Seam Testing - Geomembranes}

\subsection{Concept}

The welded seam created by a fusion welding process is composed of a primary seam and a secondary track that creates an unwelded channel. The presence of an unwelded channel permits fusion seams to be tested by inflating the sealed channel with air to a predetermined pressure and observing the stability of the pressurized channel over time.

The Contractor shall perform non-destructive air-pressure testing in accordance with the Test Method GM-6 that has been adopted by the Geosynthetic Research Institute.

\subsection{Air Pressure Testing}

\subsubsection{Equipment for Air Testing}

4.2.1.1 An air pump (manual or motor driven) capable of generating and sustaining a pressure between 20 to 60 psi.

4.2.1.2 A rubber hose with fittings and connections.

4.2.1.3 A sharp hollow needle, or other approved pressure feed device with a pressure gauge capable of reading and sustaining a pressure between 0 and 60 psi.

\subsubsection{Procedure for Air Testing}

4.2.2.1 Seal both ends of the seam to be tested.

4.2.2.2 Insert needle or other approved pressure feed device into the sealed channel created by the fusion weld.

4.2.2.3 Inflate the test channel to a pressure of approximately $30 \mathrm{psi}$, and maintain the pressure within the range listed in the Initial Pressure Schedule. Close valve, observe and record the initial pressure.

INITIAL PRESSURE SCHEDULE *
\[ \begin{array}{ccc}\text { MATERIAL } & \text { MINIMUM } & \text { MAXIMUM } \\ \text { (mils) } & \text { (psi) } & \text { (psi) } \\ 40 & 24 & 30\end{array} \]




$\begin{array}{rrr}60 & 27 & 35 \\ 80 & 30 & 35 \\ 100 & 30 & 35\end{array}$

*Initial pressure settings are recorded after an optional two minute stabilization period. The purpose of this "relaxing period" is to permit the air temperature and pressure to stabilize. The initial pressure reading may be recorded once stabilization has taken place.

4.2.2.4 Observe and record the air pressure five minutes after the initial pressure setting is recorded. If loss of pressure exceeds the maximum permissible per the manufacturer's recommendation or if the pressure does not stabilize, locate the suspect area and repair in accordance with Section 4.2.3.

4.2.2.5 At the conclusion of all pressure tests, the end of the air-channel opposite the pressure gauge is cut. A decrease in gauge pressure must be observed or the air channel will be considered "blocked" and the test will have to be repeated from the point of blockage. If the point of blockage cannot be found, cut the air channel in the middle of the seam and treat each half as a separate test.

4.2.2.6 Remove the pressure feed needle and seal the resulting hole by extrusion welding.

4.2.3 In the event of a Non-Complying Air Pressure Test, the following procedure shall be followed:

4.2.3.1 Check seam end seals and retest seams.

4.2.3.2 If a seam will not maintain the specified pressure, the seam should be visually inspected to localize the flaw. If this method is unsuccessful, cut one-inch samples from each end of the seam.

4.2.3.3 Perform destructive peel tests on the samples using the field tensiometer.

4.2.3.4 If all samples pass destructive testing remove the overlap left by the wedge welder and vacuum test the entire length of seam in accordance with Paragraph 4.3.

4.2.3.4.1 If a leak is located by the vacuum test, repair by extrusion fillet welding. Test the repair by vacuum testing. 
4.2.3.4.2 If no leak is discovered by vacuum testing, the seam will be considered to have passed non-destructive testing.

4.2.3.5 If one or more peel specimens are in non-compliance, additional samples will be taken in accordance with Paragraph 4.4.3.

4.2.3.5.1 When two passing samples are located, the length of seam bounded by the two passing test locations will be considered non-complying. The overlap left by the wedge welder will be heat tacked in place along the entire length of seam and the non-complying portion of seam will be extrusion fillet welded.

4.2.3.5.2 Test the entire length of the repaired seam by vacuum testing in accordance with Paragraph 4.3.

\subsubsection{General Air Testing Procedures}

4.2.4.1 The opposite end of the air channel will in all cases be pierced to assure that no blockages of the air channel have occurred.

4.2.4.2 Whenever possible, seams should be air-tested prior to completing butt seams to avoid having to cut into liner. All cuts through the liner as a result of testing will be repaired by extrusion welding.

4.2.4.3 All needle holes in air channels remaining after testing will be circled by testing crew and will be repaired with an extrusion bead.

\subsubsection{Air Pressure Testing Documentation}

All information regarding air-pressure testing, (date, initial time and pressure, final time and pressure, pass/fail designation, and Technicians initials) will be written at both ends of the seam, or portion of seam tested. All of the above information will also be logged by the Contractor on a NonDestructive Testing Form and submitted to the SNLA Designated Representative.

\subsection{Vacuum Testing}

This test is used on extrusion welds, or when the geometry of a fusion weld takes air pressure testing impossible or impractical, or when attempting to locate the precise location of a defect believed to exist after air pressure testing.

\subsubsection{Equipment for Vacuum Testing}


4.3.1.1 Vacuum box assembly consisting of a rigid housing with a soft neoprene gasket attached to the bottom, a transparent viewing window, port hole or valve assembly, and a vacuum gauge.

4.3.1.2 Vacuum pump assembly equipped with a pressure controller and pipe connection.

4.3.1.3 A rubber pressure/vacuum hose with fittings and connections.

4.3.1.4 A bucket and means to apply a soapy solution.

4.3.1.5 A soapy solution.

\subsubsection{Procedure for Vacuum Testing}

4.3.2.1 Trim excess overlap from the seam, if any.

4.3.2.2 Turn on the vacuum pump to reduce the vacuum box to approximately 10 inches of mercury, i.e., 5 psi gauge.

4.3.2.3 Apply a generous amount of a strong solution of liquid detergent and water to the area to be tested.

4.3.2.4 Place the vacuum box over the area to be tested and apply sufficient downward pressure to "seat" the seal strip against the liner.

4.3.2.5 Close the bleed valve and open the vacuum valve.

4.3.2.6 Apply a minimum of 5 psi vacuum to the area as indicated by the gauge on the vacuum box.

4.3.2.7 Ensure that a leak tight seal is created.

4.3.2.8 For a period of approximately 10 to 15 seconds, examine the geomembrane through the viewing window for the presence of soap bubbles.

4.3.2.9 If no bubbles appear after 15 seconds, close the vacuum valve and open the bleed valve, move the box over the next adjoining area with a minimum three inch overlap, and repeat the process.

\subsubsection{Procedure for Non-complying Test}

4.3.3.1 Mark all areas where soap bubbles appear and repair the marked areas in accordance with Paragraph 5.3. 
4.3.3.2 Retest repaired areas.

\subsubsection{General Vacuum Testing Procedures}

4.3.4.1 Vacuum box testing will be performed by qualified construction personnel with frequent supervision by the SNLA Designated Representative.

4.3.4.2 Overlap must be trimmed prior to vacuum boxing all seams.

4.3.4.3 Special attention shall be exercised when vacuum testing " $\mathrm{T}$ " seams or patch intersections with seams.

\subsubsection{Vacuum Testing Documentation}

4.3.5.1 Vacuum testing crew will use permanent markers to write on liner indicating tester's initials, date, and pass/fail designation on all areas tested.

4.3.5.2 Records of vacuum testing will be maintained by the Contractor on a Non-Destructive Testing Form or a Repair Report Form and submitted to the SNLA Designated Representative.

\subsection{Destructive Testing}

\subsubsection{Concept}

The purpose of destructive testing is to determine and evaluate seam strength. These tests require direct sampling and thus subsequent patching. Therefore, destructive testing will be held to a minimum to reduce the amount of repairs to the geomembrane.

\subsubsection{Procedure for Destructive Testing}

4.4.2.1 Destructive test samples shall be marked and cut out randomly at a minimum average frequency of one test location every 500 feet of seam length.

4.4.2.2 Location of destructive samples will be selected by the SNLA Designated Representative, with samples cut by the Contractor.

4.4.2.3 Destructive samples should be taken and tested as soon as possible after the seams are welded (the same day), in order to receive test results in a timely manner. 
4.4.2.4 The SNLA Designated Representative will observe all destructive testing. The Contractor will record the date, time, seam number, location, and test results on a Destructive Testing Form and submit to the SNLA Designated Representative.

4.4.2.5 All destructive test locations with pass/fail designation will be marked on liner with permanent Mean Streak markers.

\subsubsection{Sample Size}

4.4.2.6.1 The sample should be twelve inches wide with a seam sixteen inches long centered lengthwise in the sample. The sample may be increased in size to accommodate independent laboratory testing by the Owner, at the Owner's request, or by specific project specifications.

4.4.2.6.2 A one-inch specimen shall be cut from each end of the test seam for field testing.

4.4.2.6.3 The two one-inch wide specimens shall be tested on a field tensiometer for peel strength. If either field specimen does not pass, it will be assumed the sample would also not pass laboratory destructive testing.

The procedure outlined in Paragraph 4.4.3 shall be followed to locate passing samples to send to the laboratory.

\subsubsection{Procedure for Non-Complying Destructive Tests}

4.4.3.1 Cut additional field samples for peel testing. In the case of a field production seam, the samples must lie approximately tenfeet in each direction from the location of the initial noncomplying sample. Perform a field test for peel strength. If these field samples pass, then laboratory samples can be cut and forwarded to the laboratory for full testing.

4.4.3.1.1 If the laboratory samples pass, then reconstruct the seam between the two passing sample locations according to procedures detailed in Section 5.3. 
4.4.3.1.2 If either of the samples are still in non-compliance, then additional samples are taken in accordance with the above procedure until two passing samples are found to establish the zone in which the seam should be reconstructed.

4.4.3.2 All passing seams must be bounded by two locations from which samples passing laboratory destructive tests have been taken.

4.4.3.3 In cases of reconstructed seams exceeding 150 feet, a sample must be taken and pass destructive testing from within the zone in which the seam has been reconstructed.

4.4.3.4 All destructive seam samples shall be numbered and recorded by the Contractor on a Destructive Test Form and submitted to the SNLA Designated Representative.

\subsection{Laboratory Testing of Destructive Seam Samples}

\subsubsection{Destructive Seam Testing Laboratory}

Seam destructive samples will be sent to a testing laboratory to be approved by the SNLA Designated Representative.

\subsubsection{Acceptance Criteria}

Destructive samples will be tested for "Shear Strength" and "Peel Adhesion" (ASTM D4437). Five specimens shall be tested for each test method. Four out of the five specimens must pass for each round of peel and shear testing. In addition, four of the five individual specimens and the average of the five peel and shear tests must meet or exceed the manufacturer's strength requirements in order for the seam to pass the destructive test.

\subsection{Defects and Repairs}

5.1 The SNLA Designated Representative shall conduct a detailed walk through and visually check all seams and non-seam areas of the geomembrane for defects, holes, blisters, and signs of damage during installation.

5.2 All Contractor personnel shall, at all times, be on the lookout for any damaged areas. Damaged areas shall be marked and repaired.

\subsection{Repair Procedures}


Any portion of the geomembrane or geomembrane seam showing a flaw, or having a destructive or non-destructive test in non-compliance shall be repaired. Several procedures exist for repair and the decision as to the appropriate repair procedure shall be made by the Contractor and approved by the SNLA Designated Representative.

Procedures available for repair include the following:

5.3.1 Patching - used to repair large holes, tears, and destructive sample locations. All patches shall extend at least three inches beyond the edges of the defect and all comers of patches shall be rounded.

5.3.2 Grinding and Welding - used to repair sections of extruded fillet seams.

5.3.3 Spot Welding or Seaming - used to repair small tears, pinholes, or other minor localized flaws.

5.3.4 Capping - used to repair lengths of extrusion or fusion welded seams.

5.3.5 Extruding the exposed overlap along the length of fusion welded seams.

5.3.6 Removal of a suspect seam and replacement with of new material seamed into place.

\subsection{Verification of Repairs}

Every repair shall be non-destructively tested using the methods set out in Paragraph 4.4. Repairs which pass the non-destructive test shall be deemed acceptable. Repairs in excess of 150 feet require a destructive test. Nondestructive testing of repairs shall be logged on a Repair Report Form by the Contractor and submitted to the SNLA Designated Representative. The repair location shall be recorded on the record drawing .

\section{End of Section 02776}


SECTION 02777

\section{GEOSYNTHETIC CLAY LINER}

\section{PART 1 GENERAL}

\subsection{GENERAL}

A. This section includes the requirements for selection., installation, and protection of a geosynthetic clay liner (GCL). Adhere to the stricter of each item in this specification or the actual GCL manufacturer's specification.

1.2 RELATED WORK

A. Section 01300 Descriptive Submittals.

B. Section 02200 Earthwork: Subgrade Preparation.

\subsection{REFERENCES}

A. The publications listed below form a part of this specification to the extent referenced. The publications are referred to in the text by basic designation only. Use the latest revision date available unless otherwise indicated.

B. American Society for Testing Materials (ASTM)

1. ASTM E496, Test Method for Plate Water Absorption of Cohesive Soils.

2. ASTM D4643 (modified), Test Method for Determination of Water Content of Soil.

3. ASTM D5084, Test Method for Measurement of Hydraulic Conductivity of Saturated Porous Materials Using a Flexible Wall Permeameter (modified).

4. ASTM D35.01.81.07, Test Method for Determining the Coefficient of Soil and Geosynthetic or Geosynthetic and Geosynthetic Friction by the Direct Shear Method.

5. ASTM D3776, Test Method for Mass Per Unit Area of Textiles.

6. ASTM D4632, Test Method for Grab Breaking Load and Elongation of Geotextiles.

7. ASTM D3786, Test Method for the Mullen Burst Strength of Textiles.

C. U.S. Pharmacopoeia - National Formulary XVII, page 1210

1. USP-NF-XVII, Test Method for the Free Swell of Bentonite Clay.

D. Geosynthetics Research Institute, Drexel University

1. GRI-GCL-1, Test Method for the Confined Swell of Geosynthetic Clay Liners. 


\subsection{SUBMITTALS}

A. Pre-installation: Submit the following to the SNLA Delegated Representative for approval prior to GCL deployment.

1. Manufacturer's specification for GCL which includes properties contained in Tables 1 and 2.

2. Written certification that the GCL meets the properties listed in Tables 1 and 2.

3. Written certification that GCL manufacturer has continuously inspected GCL for the presence of needles and found GCL to be needle-free.

4. Written certification from the GCL manufacturer that the bentonite will not shift during transportation or installation thereby causing thin spots in the body of the GCL.

5. Quality control certificates signed by a responsible entity of the GCL manufacturer. Each quality control certificate shall include roll identification numbers, and results of quality control tests. At a minimum, results shall be given for tests and corresponding methods specified in Tables 1 and 2 .

B. Installation---Submit the following as installation proceeds: Subgrade surface acceptance, signed by the Contractor for each area that will be covered directly by GCL.

\subsection{DELIVERY, STORAGE, AND HANDLING}

A. Packing and Shipping

1. GCL shall be supplied in rolls wrapped individually in relatively impermeable and opaque protective covers.

2. GCL rolls shall be marked or tagged with the following information:

a. Manufacturer's name

b. Product identification

c. Roll number

d. Roll dimensions

e. Roll weight

B. Storage and Protection:

1. The Contractor will provide on-site storage area for GCL rolls from time of delivery until installed.

2. After Contractor mobilization, store and protect geotextile from dirt, water, ultraviolet light exposure, and other sources of damage.

3. Preserve integrity and readability of GCL roll labels.

\section{PART 2 PRODUCTS}

\subsection{MATERIALS}


A. The active ingredient of the GCL shall be natural sodium bentonite. The bentonite shall be encapsulated between two polypropylene textiles.

B. For side slopes steeper than 7H:IV, the GCL shall be lock-stitched with high strength polypropylene thread to provide internal shear strength reinforcing. The internal shear reinforcing mechanism shall resist failure due to thread pull-out over long-term creep situations.

C. The textiles shall be sufficiently porous to allow bentonite flow-through such that the permeability of the overlap seams is equal to or less than the permeability of the body of the GCL sheet without the addition of granular or paste bentonite.

D. The bentonite and textiles used to manufacture the GCL shall have the following properties:

\section{TABLE 1}

\begin{tabular}{|c|c|c|c|}
\hline Property & Test Method & Value & Units \\
\hline BENTONITE & & & \\
\hline Free Swell & USP-NF-XVII & 25 & \\
\hline Plate Water Absorption & ASTM E946 & 750 & $\%$ \\
\hline pH & ASTM D4972 & 8 to 11 & \\
\hline TEXTILE BACKINGS & & & \\
\hline Weight & ASTM D3776 & 3.0 & oz/sy \\
\hline Grab Tensile & ASTM D4632 & 90 & $\mathrm{lb}$ \\
\hline Mullen Burst & ASTM D3786 & 150 & $\mathrm{psi}$ \\
\hline
\end{tabular}

E. The final GCL product will have the following properties:

\section{TABLE 2}

\begin{tabular}{|c|c|c|c|c|}
\hline Property & Test Method & Value & Units & Min. Test Freq. \\
\hline $\begin{array}{c}\text { Bentonite Content 20\% } \\
\text { Moisture Content }\end{array}$ & $\begin{array}{c}\text { Weigh 12" x Roll } \\
\text { Width }\end{array}$ & 1.0 & $\begin{array}{c}\text { Ibs./sq. } \\
\mathrm{ft.}\end{array}$ & $1 / 20,000 \mathrm{sf}$ \\
\hline Confined Swell & GRI-GCL-1 & 150 & $\%$ & $1 / 300,000 \mathrm{sf}$ \\
\hline $\begin{array}{c}\text { Permeability under 5 psi } \\
\text { effective confining } \\
\text { pressure }\end{array}$ & ASTM D5084 & $5 \times 10^{-9}$ & $\mathrm{~cm} / \mathrm{sec}$ & $1 / 1,000,00 \mathrm{sf}$ \\
\hline $\begin{array}{c}\text { Overlap Seam Permeability } \\
\text { under 5 psi effective } \\
\text { confining pressure }\end{array}$ & ASTM D5084 & $5 \times 10^{-9}$ & $\mathrm{~cm} / \mathrm{sec}$ & $1 / 1,000,000 \mathrm{sf}$ \\
\hline
\end{tabular}




\begin{tabular}{|c|c|c|c|c|}
\hline $\begin{array}{l}\text { Hydrated Internal Shear } \\
\text { Resistance* }\end{array}$ & $\begin{array}{c}\text { ASTM } \\
\text { D35.01.81.07 } \\
\text { (Draft) }\end{array}$ & 10 & degrees & Pẹriodic \\
\hline $\begin{array}{l}\text { Hydrated Internal Shear } \\
\text { Resistance* }\end{array}$ & $\begin{array}{l}\text { ASTM } \\
\text { D35.01.81.07 } \\
\text { (Draft) }\end{array}$ & 500 & $\mathrm{psf}$ & Periodic \\
\hline
\end{tabular}

* For side slopes less than or equal to 7H:IV.

** For side slopes steeper than $7 \mathrm{H}: 1 \mathrm{~V}$.

F. The bentonite shall be continuously adhered to both geotextiles to ensure that the bentonite will not be displaced during handling, transportation, storage and installation, including cutting, patching and fitting around penetrations.

G. The bentonite sealing compound or bentonite granules used to seal penetrations and make repairs shall be made of the same natural sodium bentonite as the GCL and shall be as recommended by the GCL manufacturer.

\section{PART 3 MANUFACTURER}

\subsection{ACCEPTABLE MANUFACTURERS}

A. The GCL shall be CLAYMAX 200R and/or CLAYMAX 500SP as manufactured by the James Clem Corporation, 234 Gordon Road, Fairmount, GA 30139 (phone: 706/337-5316; fax: 706 337-2215) or SNLA Designated Representative's approved equal.

\subsection{MANUFACTURING EXPERIENCE}

A. The Manufacturer of the GCL shall have a minimum of five years of continuous experience in the manufacture of similar GCL products. The Manufacturer must demonstrate, by submitting a list of previous projects, a minimum of 1 million square feet of Manufacturing experience of similar GCL products.

\section{PART 4 WARRANTY}

\subsection{MATERIAL}

A. The Manufacturer shall provide a five year warranty to the Owner against manufacturing defects. The warranty shall include defective product found to be not in compliance with the requirements of Section 2.0 and Tables 1 and 2. The warranty shall include the supply of the replacement GCL material. The warranty 
shall not include the cost of re-installation, defects or failures due to improper installation.

\section{PART 5 EXECUTION}

\subsection{EXAMINATION}

A. The SNLA Delegated Representative will collect samples of GCL to be installed for conformance testing.

\subsection{SUBGRADE PREPARATION}

A. The subgrade shall be prepared in a manner consistent with proper subgrade preparation techniques for the installation of geosynthetic materials.

B. The subgrade shall be properly compacted so as not to settle and cause excessive strains in the GCL or other synthetic liner materials.

C. Ensure that rutting or ravening is not caused by installation equipment.

D. Ensure a surface free of debris, roots, or angular stones larger than $1 / 2$-inch.

E. Prior to installation, ensure that the subgrade has been rolled to provide smooth surface.

\subsection{INSTALLATION}

A. GCL Deployment: Handle GCL in a manner to ensure that it is not damaged. At a minimum, comply with the following:

1. On slopes, anchor the GCL securely and deploy it down the slope in a controlled manner.

2. Weight the GCL with sandbags or equivalent in the presence of wind .

3. Cut GCL with a geotextile cutter (hook blade), scissors, or other approved device. Protect adjacent materials from potential damage due to cutting of GCL.

4. Prevent damage to underlying layers during placement of GCL.

5. During GCL deployment, do not entrap in or beneath GCL, stones, trash, or moisture that could damage GCL.

6. Visually examine entire GCL surface. Ensure no potentially harmful foreign objects, such as needles, are present.

7. Do not place GCL in the rain or at times of impending rain.

8. Do not place GCL in areas of ponded water.

9. Replace GCL that is hydrated before placement of overlying geomembrane or a minimum of 12-inches of approved cover soil. 
10. In general, only deploy GCL that can be covered during that day by geomembrane or a minimum of 12 inches of approved cover soil.

11. For needle-punched GCLs, add granular bentonite to the overlapped areas at a rate of $1 / 4-1 b$ per linear foot.

12. Face the non-woven or secondary woven carrier geotextile against soil or geotextile filter fabric.

13. Face the woven carrier geotextile against geomembrane.

14. On side slopes, run GCL to the bottom of the slope as indicated .

B. Overlaps:

1. On slopes, overlap GCL to the manufacturer's match line.

2. In general, no horizontal seams are allowed on side slopes.

3. For needle-punched GCLs, apply granular bentonite to overlapped area at a rate required by the manufacturer.

C. Defects and Repairs:

1. Repair all flaws or damaged areas by placing a patch of the same material extending at least 1 -foot beyond the flaw or damaged area.

2. For needle-punched GCLs, add granular to the overlapped edges of the patch at a rate of $1 / 4-\mathrm{lb}$ per linear foot.

D. Interface with Other Products: Ensure the following when deploying overlying material.

1. GCL and underlying materials are not damaged.

2. Minimal slippage of GCL on underlying layers occurs.

3. No excess tensile stresses occur in GCL.

4. If necessary, approved adhesive can be used to keep overlap seams and patches in place during placement of overlying materials.

\subsection{EQUTPMENT}

A. Storage

1. Wooden pallets for above ground storage of GCL.

2. Heavy, waterproof tarpaulin for protecting unused GCL.

B. Installation

1. Front-end loader, crane or similar equipment for GCL deployment with a spreader bar to prevent slings from damaging edges.

2. 15-feet long, 3-inch outer diameter schedule 120 steel pipe to be inserted into roll core for lifting. 
3. Sand bags for securing tarpaulin.

4. 3-inch wide grips for moving GCL panels into place for each installation technician.

5. Glue for joining overlapped panels, or as dictated by project needs requirements.

6. Bentonite Sealing Compound and/or Granular Bentonite for securing around penetrations and structures as shown on the contract documents.

7. Anchor bolts for securing around concrete structures, if required.

8. Utility knives and replacement blades.

End of Section 02777 


\section{SECTION 02778}

\section{GEOTEXTILES}

\subsection{Material}

All geotextile material to be used will be "011/280" Trevira Spunbound nonwoven polyester needlepunched fabric or approved equal.

\subsection{Handling and Placement}

All geotextiles shall be handled in a manner to ensure they are not damaged. The following special handling requirements shall be adhered to:

2.1 On slopes, the geotextiles shall be secured and then rolled down the slope in such a manner as to continually keep the geotextile sheet in sufficient tension to preclude folds and wrinkles.

2.2 In the presence of wind, all geotextiles shall be weighted with sandbags or the equivalent.

2.3 Geotextiles shall be cut using an approved cutter. If the material is being cut in place, special care must be taken to protect other geosynthetic materials from damage.

2.4 Care shall be taken not to entrap stones or excessive dust that could damage the geotextile, or generate clogging of drains or filters.

\subsection{Seams and Overlaps}

Geotextiles will be seamed per the manufacturer's recommendations. No horizontal seams shall be allowed on side slopes.

3.1 On slopes steeper than ten horizontal to one vertical, it is recommended that geotextiles be continuously sewn along the entire length of the seam. Geotextiles shall be overlapped a minimum of four-inches prior to sewing.

3.2 On bottoms and slopes shallower than ten horizontal to one vertical, geotextiles can be either sewn as indicated above or thermally bonded. If thermally bonded the geotextile shall be overlapped a minimum of eight inches prior to seaming.

\subsection{Repairs}


Any holes or tears in the geotextile shall be repaired as follows:

4.1 On Slopes - A patch made from the same geotextile shall be seamed into place. Should any tear exceed $10 \%$ of the width of the roll, that roll shall be removed from the slope and replaced.

4.2 Horizontal Areas - A patch made from the same geotextile shall be spotseamed in place with a minimum of twelve inches overlap in all directions.

\section{End of Section 02778}




\section{SECTION 02779}

\section{DRAINAGE NETS}

\subsection{Manufactured Properties}

1.01 Drainage nets shall be manufactured by extruding two sets of polyethylene strands to form a three dimensional structure to provide planar water flow.

1.02 The drainage net shall contain stabilizers to prevent ultraviolet light degradation.

\subsection{Material Properties}

2.01 The drainage net shall be POLY-NET 3000 or approved equal. The drainage net shall conform to the properties detailed below:

\begin{tabular}{|c|c|c|c|c|}
\hline Property & Test Method & Unit & Qualifier & Value \\
\hline Resin Density & ASTM D1505 & $\mathrm{g} / \mathrm{cm}^{2}$ & minimum & 0.940 \\
\hline $\begin{array}{c}\text { Resin Melt } \\
\text { Index }\end{array}$ & ASTM D 1238 & $\mathrm{g} / 10 \mathrm{~min}$ & maximum & 0.5 \\
\hline $\begin{array}{c}\text { Carbon Black } \\
\text { Content }\end{array}$ & ASTM D1603 & $\%$ & minimum & 2 \\
\hline Thickness & ASTM D751 & inches & minimum & 0.200 \\
\hline $\begin{array}{c}\text { Mass per Unit } \\
\text { Area }\end{array}$ & ASTM D3776 & lbs/ft & minimum & 0.162 \\
\hline $\begin{array}{c}\text { Transmissivity } \\
\text { @ 15,000 psf }\end{array}$ & ASTM & $\mathrm{m}^{2} / \mathrm{sec}$ & minimum & $1 \times 10^{-5}$ \\
\hline $\begin{array}{c}\text { Tensile } \\
\text { Strength }\end{array}$ & ASTM D1682 & lbs/in & minimum & 42 \\
\hline $\begin{array}{c}\text { Standard Width } \\
\text { X Length }\end{array}$ & -- & feet & -- & $7.54 \times 300$ \\
\hline
\end{tabular}

(1) Per ASTM D4716-87, the transmissivity was Measured using water @ 20C (68F) with a gradient of one, between two steel plates, after one hour. Values may vary, based on dimensions of the transmissivity specimen and individual laboratory.

\subsection{Manufacturer's Statement}

3.01 The manufacturer shall submit on request, notarized certifications indicating that the material meets the above specification, signed by an authorized employee of the manufacturer.

3.02 Drainage nets shall be supplied in rolls, marked or tagged with the following information: 

1) Manufacturer's name
2) Product identification
3) Lot number
4) Roll number
5) Roll dimensions

\subsection{Installation}

4.01 Care shall be taken to keep the drainage net clean and free from debris prior to installation. If the drainage net is not clean, it should be washed prior to installation.

4.02 On slopes, drainage nets shall be properly anchored per the manufacturer's recommendations and agreed upon by the SNLA Designated Representative.

4.03 Drainage nets shall not be welded to geomembranes.

4.04 Adjacent rolls shall be overlapped approximately four inches and secured by plastic ties approximately every five-feet along the roll length. Plastic ties shall be white or other bright color for easy inspection. Metallic ties shall not be allowed.

4.05 In corners of the slopes, where overlaps between rolls of nets are staggered, an extra layer of drainage net shall be installed form the top to the bottom of the slope.

4.06 Contractor shall place all cover materials in such a manner to ensure the drainage net and underlying materials are not damaged.

End of Section 02779 


\section{Section 02780}

\section{TEXNET}

\subsection{Related Work.}
A. Section 01300 Descriptive Submittals.
B. Section 02778 Geotextiles.
C. Section 02779 Drainage Nets.

\subsection{Material and Product Construction.}

1. The product shall be TexNet by National Seal Company or approved equal.

A. The drainage net shall be as described in Section 02779 of these specifications.

B. The geotextile shall be as described in Section 02778 of these specifications.

C. The texnet shall be manufactured by heat bonding the geotextile to the HDPE drainage net on one side only. No burn through geotextiles shall be permitted. No glue or adhesive shall be permitted.

D. The bond between the geotextile and the HDPE drainage net shall exhibit a minimum average peel strength of 2 pounds per inch, and a typical peel strength of 4 pounds per inch as per ASTM D-413. Peel strength shall be tested approximately every 600 linear feet. These tests are to be performed by the installing contractor in the presence of a SNLA Designated Representative. All results are to be submitted to the SNLA Designated Representative.

The manufacturer shall submit samples of the HDPE drainage net and geotextile to the SNLA Designated Representative with a complete set of specifications for approval. The manufacturer shall also furnish a complete set of instructions for storage, handling, installation, and joining that are to be followed by the installer.

\subsection{Manufacturers Statement}

A. The manufacturer shall submit certifications indicating that the material meets the above specifications and signed by an authorized employee.

B. The TexNet shall be supplied in rolls, marked or tagged with the following:

1. Manufacturer's name, 
2. Product identification,

3. Lot number,

4. Roll number,

5. Roll dimensions.

\subsection{Installation}

A. Care shall be taken to keep the texnet clean and free from debris prior to installation. If the texnet is not free of soil and debris before installation, it shall be cleaned by the contractor just prior to installation.

B. On slopes, the texnet shall be properly anchored per manufacturer's recommendations.

C. Adjacent rolls shall be overlapped approximately 2 to 4 inches and secured by plastic ties approximately every five-feet along the roll length. Plastic ties shall be white or another bright color for easy inspection. Metallic ties shall not be allowed. The geotextile will then be either overlapped, sewn, or heat tacked per the manufacturers recommendation. The geocomposite shall not be welded to geomembranes.

D. The installer shall handle all geocomposite rolls in such a manner as to ensure they are not damaged in any way, and the following shall be complied with:

The texnet shall be secured and then rolled down the slope in such a manner as to continually keep the texnet in tension. If necessary, the texnet shall be positioned by hand after being unrolled to minimize wrinkles. The texnet cannot be placed in the horizontal direction (i.e., across the slope).

In the presence of wind, all texnet rolls in place shall be weighted with sandbags or the equivalent. Such sandbags shall be installed during placement and shall remain until replaced with cover material.

E. Contractors shall place all cover materials in such a manner as to ensure the texnet and underlying materials are not damaged.

F. The TexNet is to be installed so that the drainage net is place on the down side on top of the underlying geomembrane. The geotextile will be upward facing with the soil placed on top of it.

\section{End of Section 02780}




\section{SECTION 02900}

\section{SEEDING}

\section{PART 1 - GENERAL}

\subsection{SCOPE}

This Specification Section covers seeding. All exposed areas not covered by riprap shall be seeded as required to control erosion.

\subsection{MATERIAL STORAGE}

A. Seeds shall be stored in sealed waterproof containers in a cool, dry location and shall be kept out of direct sunlight until ready for use.

B. Fertilizer shall be delivered and stored in waterproof containers that show the chemical analysis and name of manufacturer.

PART 2 - MATERIALS

2.1 SEED MIX

A. Permanent Cover: The following seed mix shall be used for seeding of final grades:

Seeding Rate

Pure Live Seed

$\underline{\text { Seed Species }}$

(Pounds per Acre)

Crested Wheatgrass

Indian Ricegrass

Alkali Sacaton

Sand Dropseed

Four-Wing Saltbrush
3

\subsection{ACCEPTANCE OF SEED}

- Final acceptance of seed will be made by the SNLA Delegated Representative based on the following: 
Seed shall be furnished separately or in mixture in standard sealed containers with the following information provided by the seed vendor on each seed container label: (1) seed name; (2) lot number; (3) net weight; (4) percentages of purity and of germination; (5) seed coverage, in acres, on a pure live seed basis; and (6) percentage of maximum weed seed content clearly marked for each kind of seed. Seeds shall be packaged by the vendor in even acre coverage containers. The Contractor shall furnish the SNLA Delegated Representative duplicate copies of a statement by the vendor, certifying that each lot of seed has been tested by a recognized laboratory for seed testing within six months of date of delivery. This statement shall include: (1) name and address of laboratory: (2) date of test; (3) lot number for each kind of seed; and (4) results of tests as to name, percentages of purity and of germination, and percentage of weed content for each kind of seed furnished, and in case of a mixture, the proportions of each kind of seed.

\subsection{FERTILIZER}

Fertilizer shall be applied at 10 pounds per acre and shall have a 10-10-10 nutrient composition. Fertilizer shall be furnished in new, clean, and sealed containers with the name, weight, and guaranteed analysis of contents clearly marked. A liquid form of fertilizer containing the minimum percentage of available nutrients may be used.

\subsection{MULCH}

Hydro-mulch shall be applied over all seeded areas and shall consist of cereal straw. Cereal straw shall be from grain crops that are free from noxious weeds, mold, or other objectionable material. A tackifier shall also be used with the mulch. Final acceptance of mulch will be by the SNLA Designated Representative.

\subsection{WATER}

Water used in the planting or care of vegetation shall be free of oils, acids, alkalis, salts or any substance injurious to plant life.

\section{PART 3 - EXECUTION}

\subsection{PREPARATION}
A. Timing:

1. During Construction (Temporary Cover): No temporary seeding will be required.

2. Final Grades (Permanent Cover): 
a. All disturbed areas not covered by gravel or riprap shall be seeded by using the seed mix specified in Article 2.1 above. Final seeding shall be completed as soon as practical after completion of final grading. Seeding materials shall not be applied during windy weather, when the ground is excessively wet or frozen, or when snow is present.

b. Before applying seed for permanent cover of a given area, the area shall be graded as shown on the Drawings, with surfaces sloping gradually toward drainage courses and no enclosed low spots where water can accumulate. Areas to be seeded that have been damaged by erosion or other causes shall be restored prior to seeding and then cultivated to provide a reasonably firm but friable seedbed. A minimum of six-inches of surface soil shall be in a loose condition at the time of seed application.

\subsection{APPLICATION}

A. Seed for temporary cover shall be applied by hydroseeding or by a mechanical broadcaster.

B. Seed for permanent cover shall be applied by a rangeland drill to a depth of 0.5 to 0.75 inch. To the greatest extent possible, seeding shall be oriented along (parallel to) land contours.

\subsection{CARE DURING CONSTRUCTION}

The Contractor shall be responsible for protecting and caring for areas seeded before final acceptance of the work. The Contractor shall repair any damage to seeded areas caused by erosion or construction operations without additional compensation.

\subsection{PLANT ESTABLISHMENT}

The Contractor shall be responsible for watering and caring for seeded areas if planted between May and August (inclusive) and shall water at least twice a week for at least

90 days after planting. Reseeding, if required, and subsequent watering will be done by the contractor at no additional cost to Sandia.

END OF SECTION 02900 


\section{Section 02901}

\section{Sprinkler System Specifications}

1.0 Related work.
A. Section 01300 Descriptive Submittals.
B. Section 02200 Earthwork.

2.0 The sprinkler system will be installed as shown on the Contract Drawings. All materials used must be submitted to the SNLA Designated Representative for approval.

The materials outlined on the Contract Drawings are to be used (approved equals are acceptable). The following are specifications for Sprinkler System Components to be used:

\section{Sprinkler Heads}

- Rain Jet Water Smart Sprinkler Heads (3" pop-ups)

- Square Pattern - Model \# RS-10 SQ,

- Coverage of 22' X 22'

- Operating Pressure of 15 psi

- Flow rate of $4.5 \mathrm{gpm}$

- Maximum spacing of $22^{\prime}$

Digital Flowmeter: (Sandia furnished, contractor installed)

- Model MT100 by McCrometer

- 3-inch diameter

- Male threads on each end

- Stainless steel construction

- Pressure rating: 150 psi

- Maximum temperature: $160^{\circ} \mathrm{F}$

Electronic Controlled Flowmeter: (Sandia furnished, contractor installed)

- Omega FP-5300

- Polypro Housing

- Titanium Shaft

- For $1 / 2 "$ - 4" Pipes

- Weight of $75 \mathrm{lbs}$.

- Length equals 3.50"

- Output Signal: 1V p-p/fps

- Output Frequency: 5-6 Hz/fps nominal 
- Flow Rate Range: 1 to 23 fps

- Source Impedance: $8 \mathrm{~K}$ ohm

- Maximum Pressure: $180 \mathrm{PSIG}$ maximum at $68^{\circ} \mathrm{F}\left(20^{\circ} \mathrm{C}\right)$

- Maximum Temperature:

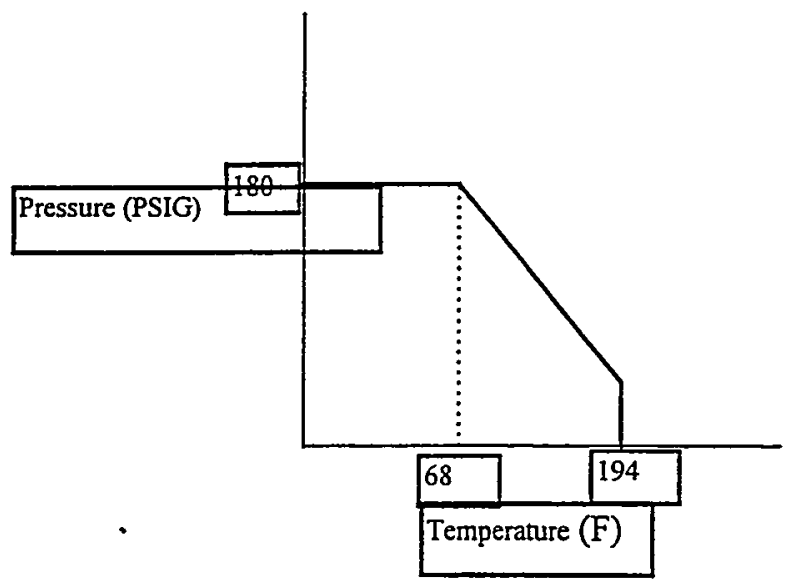

- Pressure Drop: Equal to $8 \mathrm{ft}$. of straight pipe

- Material: Transducer Housing: Glass filled polypropylene; O-Rings: Viton; Shaft: Titanium (PVDF opt.); Rotor: PVDF

- Maximum \% Solids: $1 \%$ of fluid volume, non-abrasive, non-magnetic, $<100$ micron diameter and length

- Standard Cable Length: 25 feet

- Max. Viscosity: 1 centipoise (water); up to $5 \mathrm{cp}$ above $5 \mathrm{fps}$ velocity

- Fittings Required: FP-5320 - 2" sensor installation fitting;

FMK - 31536-1 polypropylene plug

Controller (Sandia furnished, contractor installed)

- Omega DPF75 scaleable totalizer/batch controller, with RS232 output (Sandia furnished, contractor installed)

- Input Power: $110 \mathrm{Vac}$ or $\pm 15 \%$ or 11 to $15 \mathrm{Vdc} ; 220 \mathrm{Vac} \pm 15 \%$ or 11 to 15 Vdc. Max. current $=250 \mathrm{~mA} \mathrm{DC}$ or $32 \mathrm{VA}$ at rated $\mathrm{AC}$ voltage

- Output Power: (AC powered units only) + 12 Vdc @50 MA, unregulated $+25 \%$

- Rate Accuracy: $\pm 0.01 \%$ of reading +1 LSD

- K Factor: up to 5 digits

- Temperature: Operating: $+32^{\circ} \mathrm{F}$ to $+130^{\circ} \mathrm{F}$; Storage: $-20^{\circ} \mathrm{F}$ to $200^{\circ} \mathrm{F}$

- Memory: EEPROM stores data for 10 years if power is lost

- Pulse Input: Low = 0-1 Vdc; High =4-30Vdc; $10 \mathrm{Kohm}$ impedance; up to 10Khz.; $\min .0 .05 \mathrm{~Hz}$ for rate indication.

- Relays: Rated 10 Amps @ 240 Vac or 28 Vdc. SPST, solder jumper selectable for N.O. or N.C., relays latch or autorecycle after 0.1 to 99.9 seconds.

- Panel Cutout: 1.772" H * 3.622"W (DIN standard); 4.06" depth.

- Shipping Weight: $2 \mathrm{lbs}$ 
Amplifier (Sandia furnished, contractor installed)

- Omega FLSC-AMP Amplifier

2-inch Zone Valve and Motor (Sandia furnished, contractor installed)

- Valve, Hardy - part no. RB-EP-200F

- $24 \mathrm{VDC}$

\section{End of Section 02901}




\section{STANDARD SPECIFICATION \\ SECTION 03300 \\ CAST-IN-PLACE CONCRETE}

\section{PART 1 - GENERAL}

\subsection{DESCRIPTION OF WORK}

A. Section Includes: Cast-in-place concrete, including formwork, reinforcing, mix design, placement procedures, and finishes.

B. Related Sections: Refer to the following sections for related work:

1. Section 01300 Descriptive Submittals

2. Section 02200 Earthwork

3. Section 05120 Structural Steel

\subsection{REFERENCES}

A. American Society for Testing and Materials (ASTM)

A82 Specification for Steel Wire, Plain, for Concrete Reinforcement

A185 Specification for Steel Welded Wire Fabric, Plain for Concrete Reinforcement

A615 Specification for Deformed and Plain Billet - Steel Bars for Concrete Reinforcement

C33 Specification for Concrete Aggregates

C94 Specification for Ready-Mixed Concrete

C150 Specification for Portland Cement

C260 Specification for Air-Entraining Admixtures for Concrete

C309 Specification for Liquid Membrane-Forming Compounds for Curing Concrete

C494 Specification for Chemical Admixtures for Concrete

C618 Specification for Fly Ash and Raw or Calcined Natural Pozzolan for Use as a Mineral Admixture in Portland Cement Concrete 
C1107 Specification for Packaged Dry, Hydraulic-Cement Grout (nonshrink)

C1116 Specification for Fiber-Reinforced Concrete and Shotcrete

D994 Specification for Preformed Expansion Joint Filler for Concrete

D1751 Specification for Preformed Expansion Joint Filler for Concrete Paving and Structural Construction

B. Corps of Engineers (CE)

CRD C621 Specification for Non shrink Grout

\subsection{SUBMITTALS}

A. General: Submit the following items in accordance with the Conditions of Contract and Division 1, Section "Descriptive Submittals".

B. Product Data: Submit product data for the following materials and items.

1. Reinforcement

2. Forming Accessories

3. Admixtures

4. Patching Compounds

5. Hardener

6. Joint Systems

7. Curing Compounds

8. Sealants

C. Shop Drawings: Submit shop drawings for fabrication, bending and placement of concrete reinforcement. Show bar schedules, stirrup spacing, diagrams of bent bars and arrangement of reinforcement including bar overlap. Include any special reinforcement required for openings through concrete structures.

D. Laboratory Test Reports: Submit concrete materials test reports and mix design reports certifying that each material or item complies with or exceeds the specified requirements.

1.04 QUALITY ASSURANCE

A. Codes and Standards: Comply with provisions of the latest edition or revision of the following, except as otherwise indicated:

1. ACI 301 "Specifications for Structural Concrete for Buildings"

2. ACI 302 "Guide for Concrete Floor and Slab Construction"

3. ACI 304 "Guide for Measuring, Mixing, Transporting and Placing Concrete" 

4. ACI 305 "Hot Weather Concreting"
5. ACI 306 "Cold Weather Concreting"
6. ACI 308 "Standard Practice for Curing Concrete"
7. ACI 309 "Standard Practice for Consolidation of Concrete"
8. ACI 315 "Details and Detailing of Concrete Reinforcement"
9. ACI 318 "Building Code Requirements for Reinforced Concrete"
10. ACI 347 "Recommended Practice for Concrete Formwork"
11. CRSI"Manual of Standard Practice"
12. SP-66 "ACI Detailing Manual"

B. Materials and installed work may require testing and retesting, as directed by the Sandia Delegated Representative, at anytime during progress of work. Retesting of rejected materials and installed work, shall be done at Contractor's expense:

\section{PART 2 - PRODUCTS}

\subsection{FORM MATERIALS}

A. Forms shall be of metal, wood or fiberglass with sufficient thickness to withstand pressure of newly-placed concrete without bow or deflection. Forms for exposed surfaces shall be free of holes, splits, surface voids, and other defects which can affect finished concrete surface.

B. Cvlindrical Columns and Supports: Form round-section members with paper or fiber tubes, constructed of laminated plies using water-resistant adhesive with wax-impregnated exterior for weather and moisture protection. Provide units with sufficient wall thickness to resist loads imposed by wet concrete without deformation.

C. Form Coatings: Provide commercial formulation form-coating compounds that will not bond with, stain, nor adversely affect concrete surfaces, and will not impair subsequent treatments of concrete surfaces.

D. Form Ties: Use factory-fabricated, adjustable-length, removable or snapoff metal form ties, designed to prevent form deflection, and to prevent spalling concrete surfaces upon removal.

1. Unless otherwise indicated, provide ties so portion remaining within concrete after removal is at least $1 / 2 "(12.7 \mathrm{~mm})$ inside concrete for steel ties and $\mathrm{V} / 4^{\prime \prime}(6.35 \mathrm{~mm})$ for wire ties. 
2. Unless otherwise shown, provide form ties which will not leave holes larger than l" $(25.4 \mathrm{~mm})$ diameter in concrete surface.

\subsection{REINFORCING MATERIALS}

A. Reinforcing Bars: ASTM A615, deformed.

1. Provide Grade 40 bars No. 3 and 4 for stirrups and ties.

2. Provide Grade 60 bars No. 3 to 18 , except as otherwise noted.

B. Welded steel wire fabric: ASTM A185.

Furnish in flat sheets, not rolls, unless rolls are acceptable to the Sandia Delegated Representative.

C. Cold-drawn steel wire: ASTM A82.

D. Supports for Reinforcement: Provide supports for reinforcement including bolsters, chairs, spacers and other devices for spacing, supporting and fastening reinforcing bars and welded wire fabric in place. Use wire bar type supports complying with CRSI recommendations, unless otherwise indicated. Do not use wood, brick, stone, broken block or pieces of concrete, etc.

E. Fibrous Reinforcement: ASTM C1116.

\subsection{CONCRETE MATERIALS}

A. Portland Cement: ASTM C150 Types I-II and III unless otherwise specified. All types of Portland Cement for paving and sidewalks shall be "low-alkali cement."

B. Aggregates: ASTM C33

C. Water: Potable

D. Fly Ash: ASTM C618, Class F.

E. Admixtures: All admixtures shall be specified in the mix design.

1. Air-Entraining Admixture: ASTM C260

2. Water-Reducing Admixture: ASTM C494, Type A.

3. High Range-Water-Reducing Admixture (Super Plasticizer): ASTM C494, Type F or Type G.

4. Water-Reducing, Retarding Admixture: ASTM C494, Type D. 
5. Chloride-containing admixtures are not permitted.

\subsection{RELATED MATERIALS}

A. Expansion Joint Materials: Premolded strips of a bituminous mastic composition conforming to ASTM D994.

B. Non-Shrink Grout: ASTM C1107 (formerly referenced as CE CRD C621), factory pre-mixed grout, non-metallic.

C. Liquid Membrane-Forming Curing Compound: ASTM C309, Type I or I$\mathrm{D}$, Class A.

D. Chemical Hardener: Hardener shall be a colorless, aqueous solution of zinc or magnesium fluosilicate. Approved proprietary hardeners shall be delivered ready for use in the manufacturer's original containers.

E. Bonding Compound: Polyvinyl acetate, rewettable type.

\subsection{CONCRETE MIX DESIGN}

A. General

1. Unless otherwise specified, the minimum strength of concrete shall be $3000 \mathrm{psi}(210.9 \mathrm{kgs}-\mathrm{sq} \mathrm{cm})$ at 28 days.

2. Concrete mixtures shall produce the necessary workability in fresh concrete and the desired strength.

3. Unless otherwise approved or specified, all concrete shall be "ReadyMixed" in accordance with ASTM C94.

4. The water-cement ratio selected shall be that required to produce a 28day strength corresponding to an over designed mix which is supported by sufficient experience data to assure that test results will fall within the limits established in this specification. Unless otherwise specified, the following proportions apply:

\begin{tabular}{|c|c|c|c|}
\hline $\begin{array}{l}\text { Strength } \\
\text { psi }\end{array}$ & $\begin{array}{l}\text { Min. Cement } \\
\text { Bag/C.Y. }\end{array}$ & $\begin{array}{c}\text { Max. W/C } \\
\text { Ratio }\end{array}$ & $\begin{array}{c}\text { Max. Water } \\
\text { Gal./Bag Cement }\end{array}$ \\
\hline $\begin{array}{l}3000(210.9 \mathrm{kgs}-\mathrm{sq} \mathrm{cm} \\
4000(281.2 \mathrm{kgs}-\mathrm{sq} \mathrm{cm}\end{array}$ & $\begin{array}{l}\text { m) } 5.0 \\
\text { m) } 6.0\end{array}$ & $\begin{array}{l}0.60 \\
0.50\end{array}$ & $\begin{array}{l}6.8 \\
5.6\end{array}$ \\
\hline
\end{tabular}

5. Allowable Slump:

\begin{tabular}{|c|c|}
\hline Allowable Slump: & $\min -\max ($ in.) \\
\hline $\begin{array}{l}\text { Massive section, pavement or slabs on ground } \\
101.6 \mathrm{~mm} \text { ) }\end{array}$ & $1-4(25.4$ \\
\hline $\begin{array}{l}\text { Slabs, beams, walls and columns } \\
\mathrm{mm} \text { ) }\end{array}$ & $3-5 \quad(76.2 \mathrm{~mm}-127$ \\
\hline $\begin{array}{l}\text { Concrete containing super plasticizer } \\
203.2 \mathrm{~mm} \text { ) }\end{array}$ & $3-8 \quad(76.2$ \\
\hline
\end{tabular}


B. Aggregate: Aggregate Grading shall be as follows for the different strengths of concrete.

\section{Coarse Aggregate}

Shall be as per ASTM C33, Table 2, Grading Requirements for Coarse Aggregates.

\section{Fine Aggregate}

Shall be as per ASTM C33, Sieve Analysis, Fine Aggregate.

C. Admixtures

1. Use water-reducing admixture or high range water-reducing admixture (super plasticizer) in all concrete.

2. Use air-entraining admixture in exterior exposed concrete unless otherwise indicated. Use an air content of 3.5 to 6.5 percent.

3. When an air-entraining admixture is used solely for increasing the workability of the mix, use an air content of 3 to 5 percent.

4. Fly Ash - Unless otherwise specified, a maximum of 9 pounds $(4.08 \mathrm{~kg}$ ) of fly ash per bag of cement will be permitted.

D. High early strength concrete shall have a compressive strength at 7 days equal to that specified for ordinary concrete at 28 days.

\section{PART 3 - EXECUTION}

\subsection{FORMS}

A. Design. erect. support. brace and maintain formwork to support vertical and lateral loads that might be applied until such loads can be supported by concrete structure. Construct formwork so concrete members and structures are of correct size, shape, alignment, elevation and position. Provide for openings, keyways, chamfers, inserts and other features required in work. Maintain formwork construction tolerances, unless otherwise indicated:

1. Variation from Plumb:

a. In the lines and surfaces of columns, piers, walls, and in arises:

In any $10 \mathrm{ft}(3.05 \mathrm{~m})$ of length $1 / 4$ in. $(6.4 \mathrm{~mm})$ Maximum for the entire length 1 in. $(25.4 \mathrm{~mm})$

b. For exposed corner columns, control-joint grooves, and other conspicuous lines:

In any $20 \mathrm{ft}(6.10 \mathrm{~m})$ length $1 / 4$ in. $(6.4 \mathrm{~mm})$ 
2. Variation from the level or from the grades specified in the contract drawings:

a. In slab soffits, ceilings, beam soffits and in arises, measured before removal of supporting shores:

In any $10 \mathrm{ft}(3.05 \mathrm{~m})$ of length $1 / 4$ in. $(6.4 \mathrm{~mm})$

In any bay or in any $20 \mathrm{ft}(6.10 \mathrm{~m})$ length $3 / 8$ in. $(9.7 \mathrm{~mm})$ Maximum for the entire length $3 / 4$ in. $(19 \mathrm{~mm})$

b. In exposed lintels, sills, parapets, horizontal grooves, and other conspicuous lines:

In any bay or in $20 \mathrm{ft}(6.10 \mathrm{~m})$ length $1 / 4$ in. $(6.4 \mathrm{~mm})$ Maximum for the entire length

3. Variation of the linear building lines from established position in plan and related position of columns, walls, and partitions:

In any bay $1 / 2 \mathrm{in.}(12.7 \mathrm{~mm})$

In any $20 \mathrm{ft}(6.10 \mathrm{~m})$ of length $1 / 2$ in. $(12.7 \mathrm{~mm})$ Maximum for the entire length 1 in. $(25.4 \mathrm{~mm})$

4. Variation in the sizes and location of sleeves, floor openings, and wall openings $1 / 4$ in. $(6.4 \mathrm{~mm})$

5. Variation in cross-sectional dimensions of columns and beams and in the thickness of slabs and walls:

Minus $1 / 4$ in. $(6.4 \mathrm{~mm})$

Plus. $1 / 2$ in. $(12.7 \mathrm{~mm})$

6. Footings*:

a.. Variations in dimensions in plan:

Minus $1 / 2$ in. $(12.7 \mathrm{~mm})$

Plus 2 in. $(50.8 \mathrm{~mm})$

b. Misplacement or eccentricity:

2 percent of the footing width in the direction of misplacement but not more than .2 in. $(50.8 \mathrm{~mm})$

c. Thickness:

Decrease in specified thickness Increase in specified thickness $5 \%$ No limit 
7. Variation in Steps:

a. In a flight of stairs:

Rise $+1 / 8$ in. $(+3.2 \mathrm{~mm})$

Tread $+1 / 4$ in. $(+6.4 \mathrm{~mm})$

b. In consecutive steps:

Rise. $+1 / 16$ in. $(+1.5 \mathrm{~mm})$ Tread $+1 / 8$ in. $(+3.2 \mathrm{~mm})$

* Tolerances apply to concrete dimensions only.

B. Design and fabricate formwork to be readily removable without impact, shock or damage to cast-in-place concrete surfaces and adjacent materials.

C. Chamfer exposed corners and edges as indicated in the drawings to produce uniform smooth lines and tight edge joints.

D. All curb and combined curb and gutter shall be divided into blocks or stones in lengths not to exceed 6 feet $(1.83 \mathrm{~m})$ long using metal templates not less than $1 / 16$ inch $(1.5 \mathrm{~mm})$ thick cut to the same cross section as the curb or curb and gutter being constructed. Templates shall be securelv attached to forms to prevent movement during concrete placement.

E. Provisions for Other Trades: Provide openings in concrete formwork to accommodate work of other trades. Determine size and location of openings, recesses and chases from trades providing such items. Accurately place and securely support items built into forms.

\subsection{PLACING REINFORCEMENT}

A. Comply with CRSI's recommended practice for "Placing Reinforcing Bars", for details and methods of reinforcement placement and supports, and as herein specified. Field bending of grade 60 bars is not permitted.

B. Clean reinforcement of loose rust and mill scale, earth, ice, oil, concrete splatter from previous pours, and other materials which reduce or destroy bond with concrete.

C. Accurately position. support and secure reinforcement against displacement by formwork, construction, or concrete placement operations. Locate and support reinforcing by metal chairs, runners, bolsters, spacers, and hangers, as required.

D. Install welded wire fabric of same gage in as long of lengths as practicable. Lap adjoining pieces at least one full mesh and lace splices with wire. Offset end laps of adjacent widths to prevent continuous laps in either direction.

E. Provide minimum cover for reinforcement of cast-in-place concrete, unless otherwise indicated. 
Concrete cast against and permanently exposed to earth..........3 in. (76.2 mm)

Concrete exposed to earth or weather

$\# 6$ and larger 2 in. $(50.8 \mathrm{~mm})$

\#5 and smaller $11 / 2$ in. $(38.1 \mathrm{~mm})$

Concrete not exposed to weather or in contact with earth

Slabs, walls, joists $3 / 4 \mathrm{in} .(19 \mathrm{~mm})$

Beams, columns $11 / 2$ in. $(50.8 \mathrm{~mm})$

\subsection{JOINTS}

\section{A. Construction Joints}

1. Locate construction joints on slab floor, which are not shown on drawings, and notify Sandia Delegated Representative for approval.

2. Provide keyways at least $1-1 / 2 "(38.1 \mathrm{~mm})$ deep in construction joints in walls and between walls and footings; accepted preformed keyways designed for this purpose may be used for slabs.

3. Place construction joints perpendicular to the main reinforcement. Continue reinforcement across construction joints.

B. Isolation Joints: Construct isolation joints in slabs-on-ground at points of contact between slabs on ground and foundations as shown on drawings. Unless otherwise indicated, install 90\# felt paper between slab and vertical surface.

\section{Contraction (Control) Joints}

1. Contraction Joints in Slabs-on-Ground: Construct contraction joints in slabs-on-ground to form panels of patterns as shown. Contraction joints in floor slabs shall be formed with preformed metal bulkheads.

2. Walks: Contraction joints shall not exceed intervals of 6 feet $(1.828 \mathrm{~m})$. Joints shall be made at regular intervals along the line of the work. On straight work, the joints shall be parallel with and at right angles to the line of the work; at curves the joints shall, in general, be along lines concentric with and radial to the proportion of the work in which they are placed. The markings shall be made with jointer tools that will round the edges of the scoring lines to a radius of $1 / 8$ inch $(3.2 \mathrm{~mm})$, with a depth of not less than $11 / 4$ inch $(31.75 \mathrm{~mm})$. The finished joint opening, exclusive of radii, shall not be wider than $1 / 8$ inch $(3.2 \mathrm{~mm})$.

\section{Expansion Joints}

1. Slabs-on-Ground: Expansion joint material shall be placed around utility access openings within the slab, including clean outs and utility valves, and between new concrete slab and adjacent masonry: Joint material shall be $1 / 2$ inch $(12.7 \mathrm{~mm})$ thick, premolded, asphalt impregnated complying with ASTM D1751 and extend the full depth of the concrete. 
2. Curbs and Gutters: Construct $1 / 2$ inch $(12.7 \mathrm{~mm})$ joints in curbs and gutters at the end of all returns except where cross gutters are being constructed. They shall be at the ends of the cross gutter transitions and also along the line of work at regular intervals, not to exceed 36 feet $(10.97 \mathrm{~m})$. Joints in gutter shall be continuous with those in adjacent curb.

3. Walks: Place joints in walks at the point of tangency (PT) and point of curvature (PC) of all walk returns, between walk and a building or structure, in walk returns, between the walk and the back of the curb returns and around all utility poles. At the PT and PC and around utility poles, the joint filler strips shall extend the full depth of the concrete being placed. Joint filler strips between walk and curb shall be the full depth of the walk with the top of the filler strip set flush with the top of the curb. Transverse sidewalk joints shall not exceed 30 feet $(9.14 \mathrm{~m})$ spacing. Joint material shall be $1 / 2$ inch $(12.7 \mathrm{~mm})$ thick.

\subsection{PREPARATIONS FOR PLACING CONCRETE}

A. Water shall be removed from excavations. Wood chips, shavings, and hardened concrete shall be removed from the forms before any concrete is placed. All equipment shall be cleaned. Forms shall be wetted, except in freezing weather, or oiled.

B. The earth shall be uniformly moist when the concrete is placed. The method of sprinkling shall not be such as to form mud or pools of water. Watering the subgrade immediately prior to placing the concrete is not sufficient to make the soil uniformly moist.

C. Notify other crafts to permit installation of their work. Coordinate the installation of joint materials and moisture barriers with placement of forms and reinforcing steel.

\subsection{PLACING CONCRETE}

A. Field Inspection

1. Concrete shall not be placed until forms and reinforcing steel have been inspected and approved.

2. Ready-Mix concrete shall be placed within a specified delivery time:

$\begin{array}{ll}\text { Below } 40^{\circ} \mathrm{F}\left(4.4^{\circ} \mathrm{C}\right) & \text { see cold weather placing } \\ 40^{\circ}-85^{\circ} \mathrm{F}\left(4.4-29.4^{\circ} \mathrm{C}\right) & 90 \text { minutes } \\ 86^{\circ}-90^{\circ} \mathrm{F}\left(30^{\circ}-32.2^{\circ} \mathrm{C}\right) & 75 \text { minutes } \\ \text { above } 90^{\circ} \mathrm{F}\left(32.2^{\circ} \mathrm{C}\right) & 60 \text { minutes }\end{array}$

Concrete exceeding delivery time may be rejected by the Sandia Delegated Representative.

3. Adding Water: No water shall be added after the initial introduction of mixing water for the batch except, when on arrival at the job site, the 
slump of the concrete is less than that specified, and the maximum water/cement ratio for the mix has not been exceeded. Such additional water shall be added to bring the slump within limits. The drum shall be turned at least 30 additional revolutions at mixing speed. The Sandia Delegated Representative shall be notified before adding any water. Water shall not be added to the batch at any later time.

The contractor shall insure that the concrete strength meets specifications and that the maximum amount of water as noted in the CONCRETE MIX DESIGN is not exceeded. Comply with ASTM C 94.

B. General: Comply with ACI 304, and as herein specified. Deposit concrete continuously or in layers of such thickness that no concrete will be placed on concrete which has hardened sufficiently to cause the formation of seams or planes of weakness. If a section cannot be placed continuously, provide construction joints as herein specified. Deposit concrete as nearly as practicable to its final location to avoid segregation.

\section{Placing Concrete in Forms}

1. Deposit concrete in forms in horizontal layers not deeper than 24 " $(609.6$ $\mathrm{mm}$ ) and in a manner to avoid inclined construction joints. Where placement consists of several layers, place each layer while preceding layer is still plastic to avoid cold joints.

2. Consolidate placed concrete by high frequency mechanical vibrating equipment, supplemented, as necessary, by hand- spading, rodding or tamping. Use equipment and procedures for consolidation of concrete in accordance with ACI 309.

3. Do not use vibrators to transport concrete inside forms. Insert and withdraw vibrators vertically at uniformly spaced locations not farther than visible effectiveness of machine. Place vibrators to rapidly penetrate placed layer and at least 6" $(152.4 \mathrm{~mm})$ into preceding layer. Do not insert vibrators into lower layers of concrete that have begun to set. At each insertion limit duration of vibration to time necessary to consolidate concrete and complete embedment of reinforcement and other embedded items without causing segregation of mix.

4. Concrete shall not be allowed to free fall more than 5 feet $(1.52 \mathrm{~m})$ unless confined by a closed chute, except that concrete being placed in walls 10 inches $(254 \mathrm{~mm})$ or less in thickness may free fall a maximum of 8 feet $(2.44 \mathrm{~m})$.

\section{Placing Concrete Slabs}

1. Deposit and consolidate concrete slabs in a continuous operation, within limits of construction joints, until the placing of a panel or section is completed.

2. Bring slab surfaces to correct level as above, and use bull floats or darbies to smooth surface, free of humps or hollows. Do not use tools (i.e., jitterbugs) that force the aggregate away from the surface. 
3. Maintain reinforcing in proper position during concrete placement operations.

E. Cold Weather Placing: Protect concrete work from physical damage or reduced strength which could be caused by frost, freezing actions, or low temperatures; comply with ACI 306 and these specifications.

1. Concrete shall be mixed and placed only when the temperature is at least $40^{\circ} \mathrm{F}\left(22^{\circ} \mathrm{C}\right)$ and rising unless permission to pour is obtained from the Sandia Delegated Representative.

2. Do not use frozen materials or materials containing ice or snow. Do not place concrete on frozen subgrade or on subgrade containing frozen materials.

3. Do not use calcium chloride, salt and other materials containing antifreeze agents or chemical accelerators.

4. When approval is obtained to place concrete at or below an atmospheric temperature of $40^{\circ} \mathrm{F}\left(22^{\circ} \mathrm{C}\right)$, the water or aggregates, or both, shall be heated, and suitable enclosures and heating devices shall be provided. The mixed concrete shall have a temperature of at least $50^{\circ} \mathrm{F}\left(28^{\circ} \mathrm{C}\right)$ and not more than $90^{\circ} \mathrm{F}\left(50^{\circ} \mathrm{C}\right)$ at the time of placing.

The heating equipment or methods shall be capable of heating the water and aggregates uniformly, and these materials shall not be heated to a temperature exceeding $150^{\circ} \mathrm{F}\left(83^{\circ} \mathrm{C}\right)$.

5. After any concrete is placed, the Contractor shall provide suitable measures to maintain a concrete surface temperature of $40^{\circ} \mathrm{F}\left(22^{\circ} \mathrm{C}\right)$ or above for a period of not less than 7 days.

F. Hot Weather Placing: When hot weather conditions exist that would seriously impair quality and strength of concrete, place concrete in compliance with ACI 305 and as herein specified.

1. Cool ingredients before mixing to maintain concrete temperature at time of placement below $90^{\circ} \mathrm{F}\left(50^{\circ} \mathrm{C}\right)$.

2. Cover reinforcing steel with water-soaked burlap when required to ensure that steel temperature will not exceed the ambient air temperature immediately before embedment in concrete.

3. Wet forms thoroughly before placing concrete.

4. Use water-reducing retarding admixture (Type D) when required by high temperatures, low humidity, or other adverse placing conditions.

\subsection{CONCRETE FINISHING .}

\section{A. General}


1. Do not use tools (i.e., jitterbugs) that force the aggregate away from the surface.

2. Water shall not be sprayed or sprinkled onto a concrete surface to aid in finishing.

3. The finisher should avoid bringing more water than necessary to the surface and avoid working the surface any more than necessary to obtain the required finish.

B. Slab Finishes

1. Float Finish: Apply float finish to slabs for driveways, exterior slabs, curbs and gutters and interior floor slabs receiving additional covering.

After screeding, consolidating, and leveling concrete slabs, do not work surface until ready for floating. Begin floating, using float blades or float shoes only, when surface water has disappeared, when concrete has stiffened sufficiently to permit operation of powerdriven floats, or both. Consolidate surface with power-driven floats or by hand-floating if area is small. Check and level surface plane to a tolerance of Class B. Class B tolerances shall be true planes within $1 / 4$ inch $(6.4 \mathrm{~mm})$ in $10 \mathrm{ft}$. $(3.05 \mathrm{~m})$, as determined by a 10 -ft. $(3.05$ $\mathrm{m})$ straightedge placed anywhere on the slab in any direction.

2. Trowel Finish: Apply trowel finish to interior exposed floors.

After floating, begin first trowel finish operation. Begin final troweling when surface produces a ringing sound as trowel is moved over surface. Consolidate concrete surface by final hand-troweling operation, free of trowel marks, uniform in texture and appearance and with surface leveled to a Class B tolerance.

3. Light Broom: For sidewalks apply trowel finish as specified above. Immediately follow with slightly scarifying surface by fine brooming perpendicular to main traffic route.

C. Finish of Formed Surfaces

1. Grout-Cleaned Finish: The entire surface of all exposed exterior concrete shall be given a rubbed grout clean-down finish using a mix of one part white Portland cement and 1-1/2 parts of fine sand mixed with sufficient water to form a grout having the consistency of thick paint. Apply to damp surface and rub down in such a manner as to obtain a smooth, filled surface uniform in color and free from defects and blemishes.

2. Smooth Rubbed Finish: Interior exposed surfaces (where scheduled to be painted) shall be rubbed with carborundum stones and water to a smooth, even surface for painting.

\subsection{CONCRETE CURING}


A. General: Immediately after placing or finishing, and as soon as the operation will not mar the finish, concrete surfaces not covered by forms shall be protected against moisture loss. Curing shall be accomplished by one of the following methods or a combination thereof, as approved, and such protection shall be maintained for a period of at least 7 days. Where formed surfaces are cured in the forms, the forms shall be kept continually wet. If the forms are removed before the end of the curing period, curing shall be continued as on unformed surfaces, using curing materials specified herein. Surfaces shall be kept free of foot and vehicular traffic during the curing period.

B. Curing Methods

1. Polyethylene Coated Burlap Mats: Surfaces shall be covered with the specified mat lapped 12 inches $(304.8 \mathrm{~mm})$. The mat shall be weighted to prevent displacement. Tears or holes shall be immediately repaired by patching.

2. Membrane Forming Curing Compound: This compound shall be applied in a two coat continuous operation, using not less than the manufacturer's recommended rate of application. If unknown, it shall be applied at 1 gallon (3.79 liters) per 200 square feet (18.58 square meters) for each coat. Surfaces damaged by construction operations during curing shall be resprayed at the same rate.

3. Water Curing: Water curing shall be used on surfaces that are to receive additional concrete or concrete fill, dustproofing and hardening treatments, stucco, plaster, or painting.

\subsection{REMOVAL OF FORMS}

The forms for any portion of the structure shall not be removed until the concrete is strong enough to not be damaged when the forms are removed.

A. If field operations are not controlled by cylinder tests, the following periods, exclusive of days when the temperature is below $40^{\circ} \mathrm{F}\left(4.4^{\circ} \mathrm{C}\right)$, may be used as a guide for removal of forms and supports:

Centering under beams
Elevated floor slabs
Walls
Columns

Sides of beams and all other parts
14 days

7-14 days*

12-24 hours*

$1-7$ days*

12-24 hours*

*The longer time dictates unless the Sandia Delegated Representative approves the lesser time.

B. If field operations are controlled by beam or cylinder tests, forms may be removed from centering under beams and floor slabs when a 2500 psi 
( $175.77 \mathrm{kgs}-\mathrm{sq} \mathrm{cm}$ ) compressive strength is attained, and it is approved by the Sandia Delegated Representative.

C. Superimposed loads shall not be placed on or against load carrying members until a $2500 \mathrm{psi}(175.77 \mathrm{kgs}-\mathrm{sq} \mathrm{cm})$ compressive strength has been attained and it is approved by the Sandia Delegated Representative.

\subsection{REUSE OF FORMS}

Clean and repair surfaces of forms to be reused in work. The shape, strength, rigidity, water-tightness, and surface smoothness of reused forms shall be maintained at all times. Any warped or bulged lumber shall be re-sized before being used. Unsatisfactory forms shall not be used.

\subsection{CONCRETE SURFACE REPAIRS}

A. Patching Defective Areas: Immediately after removing forms, cut back form ties and remove all fins and loose material. Honeycombs and voids shall be cut to solid concrete, thoroughly wetted, and filled with cement mortar composed of one part cement to three parts sand.

B. Repair of Formed Surfaces: Remove and replace concrete having defective surfaces if defects cannot be repaired to satisfaction of Inspector. Surface defects include color and texture irregularities, cracks, spalls, air bubbles, honeycomb, rock pockets, fins and other projections on surface, and stains and other discolorations that cannot be removed by cleaning. Flush out form tie holes, fill with dry-pack mortar.

C. Repair of Slabs-on-Ground: Test surfaces for smoothness and verify surface plane to tolerances specified for each surface and finish. Correct low areas during or immediately after completion of surface finishing operations by cutting out low areas and replacing with patching compound. Finish repaired areas to blend into adjacent concrete. Proprietary underlayment compounds may be used when accepted per 1.04 of this specification. Correct high areas in slabs by grinding after concrete has cured at least 14 days.

\subsection{CONCRETE TRUCK DISCHARGE}

A. Excess Concrete: Excess concrete in mixer trucks that cannot be immediately used shall be discharged to an area where it will not create an obstruction or hazard during construction. This concrete shall be removed from the site in a timely manner to a site approved by the Sandia Delegated Representative.

B. Wash Water Discharge: Wash water from mixer trucks shall be discharged to the ground surface in such a manner and at such a location that discharge can not escape the construction site or be washed away to arroyos, storm sewers, or sanitary sewers by precipitation or other surface flows. Prior to project completion, wash water residue shall be removed from the site to a 
location approved by the Sandia Delegated Representative and the wash water discharge site shall be cleaned free of debris.

END OF SECTION 03300 


\section{STANDARD SPECIFICATION \\ SECTION 05120 \\ STRUCTURAL STEEL}

\section{PART I - GENERAL}

\subsection{DESCRIPTION OF WORK}

A. This section includes fabrication and erection of structural steel work, as shown on drawings including schedules, notes, and details showing size and location of members, typical connections, and types of steel required.

Structural steel is that work defined in American Institute of Steel Construction (AISC) "Code of Standard Practice for Steel Buildings and Bridges" and as otherwise shown on drawings.

B. Related Sections: Refer to the following sections for related work:

1. Division 3, Section "Cast-In-Place Concrete" for anchor bolt and reinforcing steel installation in concrete.

2. Division 5, Section "Metal Fabrications" for miscellaneous metal fabrications.

3. Division 9, Section "Painting" for priming and painting of steel.

\subsection{REFERENCES}

A. American Societv of Testing and Materials (ASTM)

A6 Specification for General Requirements for Delivery of Rolled Steel Plates, Shapes, Sheet Piling and Bars for Structural Use

A27 Specification for Steel Castings, Carbon, for General Application

A36 Specification for Structural Steel

A53 Specification for Pipe, Steel, Black and Hot-Dipped, Zinc-Coated Welded and Seamless

A108 Specification for Steel Bars, Carbon, Cold-Finished, Standard Quality 
A153 Specification for Zinc Coating (Hot-Dip) on Iron and Steel Hardware

A307 Specification for Carbon Steel Bolts and Studs, 60000 psi Tensile Strength

A325 Specification for High-Strength Bolts for Structural Steel Joints

A500 Specification for Cold-Formed Welded and Seamless Carbon Steel Structural Tubing in Rounds and Shapes

A501 Specification for Hot-Formed Welded and Seamless Carbon Steel Structural Tubing

B695 Specification for Coatings of Zinc Mechanically Deposited on Iron and Steel

C1107 Specification for Packaged Dry, Hydraulic-Cement Grout (Nonshrink)

E94 Guide for Radiographic Testing

E142 Method for Controlling Quality of Radiographic Testing

E164 Practice for Ultrasonic Contact Examination of Weldments

E165 Practice for Liquid Penetrant Inspection Method

E709 Practice for Magnetic Particle Examination

F959 Specification for Compressible-Washer-Type Direct Tension Indicators for Use with Structural Fasteners

B. American Welding Societv (AWS)

D1.1 Structural Welding Code - Steel

C. Corps of Engineers (CE)

CRD C621 Specification for Nonshrink Grout

D. Federal Specification (FS)

TT-P-664 Primer Coating, Alkyd, Corrosion-Inhibiting, Lead and Chromate Free, VOC-Compliant

E. National Fire Protection Assoc. (NFPA) 
51B Standard for Fire Prevention in Use of Cutting and Welding Processes

F. Steel Structures Painting Council (SSPC)

SP-1 Solvent Cleaning

SP-2 Hand-Tool Cleaning

SP-3 Power-Tool Cleaning

\subsection{SUBMITTALS}

A. General: Submit the following in accordance with conditions of Contract and Division 1, Section "Descriptive Submittals".

B. Product Data: Submit product data or manufacturer's specifications and installation instructions for the following products. Include laboratory test reports and other data to show compliance with specifications (including specified standards).

1. Structural steel (each type), including certified copies of mill reports covering chemical and physical properties.

2. Anchor bolts.

3. Unfinished threaded fasteners.

4. High-strength bolts (each type), including nuts and washers; include direct tension indicators if used.

5. Structural steel primer paint.

6. Nonmetallic shrinkage-resistant grout.

C. Material Safety Data Sheets (MSDS): Submit MSDS for structural steel (each type), anchor bolts, unfinished threaded fasteners, high-strength bolts (each type) including nuts and washers, structural steel primer paint and nonmetallic shrinkage-resistant grout.

D. Shop drawings: Submit shop drawings, including complete details and schedules for fabrication and assembly of structural steel members, procedures, and diagrams. 
1. Include details of cuts, connections, cambers, holes and other pertinent data. Indicate welds by standard AWS symbols and show size, length, and type of each weld.

2. Provide setting drawings, templates, and directions for installation of anchor bolts and other anchorages to be installed as work of other sections.

E. Welder Certifications: Provide certification that welders to be employed in work have satisfactorily passed qualification tests in accordance with AWS D1.1.

If recertification of welders is required, retesting will be Contractor's responsibility.

F. Test reports: Submit test reports conducted on shop- and field-bolted and welded connections. Include data on type(s) of tests conducted and test results.

\subsection{QUALITY ASSURANCE}

A. Codes and Standards: Comply with provisions of the following, except as otherwise indicated:

1. AISC "Code of Standard Practice for Steel Buildings and Bridges" with paragraph 4.2.1 modified by deletion of the following sentence:

"This approval constitutes the owner's acceptance of all responsibility for the design adequacy of any detail configuration of connections developed by the fabricator as a part of his preparation of these shop drawings."

2. AISC "Specifications for Structural Steel Buildings" including the "Commentary", later referred to as "AISC Specifications".

3. "Specifications for Structural Joints using ASTM A325 or A490 Bolts" approved by the Research Council on Structural Connections.

4. AWS D1.1

5. ASTM A6

B. Qualifications for Welding Work: Qualify welding procedures and welding operators in accordance with the requirements of AWS D1.1. 
C. All materials used shall not contain asbestos fibers.

\subsection{DELIVERY. STORAGE AND HANDLING}

A. Deliver materials to site at such intervals to ensure uninterrupted progress of work.

B. Deliver anchor bolts and anchorage devices, which are to be embedded in cast-in-place concrete or masonry, in ample time so that work will not be delayed.

C. Store materials to permit easy access for inspection and identification. Keep steel members off ground by using pallets, platforms, or other supports. Protect steel members and packaged materials from corrosion and deterioration. If bolts and nuts become dry or rusty, clean and lubricate before use.

Do not store materials on structure in a manner that might cause distortion or damage to members or supporting structures. Repair or replace damaged materials or structures as directed.

\subsection{PROJECT CONDITIONS}

Field Measurements: Check actual locations of walls and other construction to which steel framing must fit, by accurate field measurements before fabrication; show recorded measurements on final shop drawings.

\section{PART 2 - PRODUCTS}

\subsection{MATERIALS}

A. Metal Surfaces. General: For fabrication of work that will be exposed to view, use only materials that are smooth and free of surface blemishes including pitting, rust and scale seam marks, roller marks, rolled trade names, and roughness. Remove such blemishes by grinding, or by welding and grinding, prior to cleaning, treating, and applying surface finishes.

B. Structural Steel Shapes. Plates. and Bars: ASTM A36 
C. Cold-Formed Steel Tubing: ASTM A500, Grade B.

D. Hot-Formed Steel Tubing: ASTM A501.

E. Steel Pipe: ASTM A53, Type E or S, Grade B: or ASTM A501.

Finish: Black, except where indicated to be galvanized.

F. Steel Castings: ASTM A27, Grade 65-35, medium-strength carbon steel.

G. Headed Stud-Type Shear Connectors: ASTM A108, Grade 1015 or 1020, cold-finished carbon steel with dimensions complying with AISC Specifications.

H. Anchor Bolts: ASTM A307, nonheaded type unless otherwise indicated.

I. Unfinished Threaded Fasteners: ASTM A307, Grade A, regular low-carbon steel bolts and nuts; provide hexagonal heads and nuts for all connections.

J. High-Strength Threaded Fasteners: Heavy hexagon structural bolts, heavy hexagon nuts, and hardened washers, as follows:

1. Quenched and tempered medium-carbon steel bolts, nuts and washers, complying with ASTM A 325 .

2. Where indicated as galvanized, provide units that are zinc-coated, either mechanically deposited complying with ASTM B695, Class 50, or hotdip galvanized complying with ASTM A153.

K. Direct Tension Indicators: ASTM F959, type as required; use at Contractor's option.

L. Electrodes for Welding: Comply with AWS Code.

M. Structural Steel Primer Paint: Red oxide, lead- and cadmium-free, corrosion-inhibiting primer complying with performance requirements of FS TT-P-664.

N. Nonmetallic Shrinkage-Resistant Grout: Premixed, nonmetallic, noncorrosive, nonstaining product containing selected silica sands, Portland cement, shrinkage compensating agents, plasticizing and water-reducing agents, complying with ASTM C1107. (formerly referenced as CE CRD C621). 
Subject to compliance with requirements, products that may be incorporated in the work include, but are not limited to, the following:

100 Non-Shrink Grout (Non-Metallic) - Conspec, Inc.

Crystex - L \& M Construction Chemicals, Inc.

Euco N-S Grout - Euclid Chemical Co.

Kemset - Chem-Masters Corp.

Sonogrout - Sonneborn Building Products Div., Rexnord Chemical Products, Inc.

Supreme Grout - Cormix, Inc.

Sure-Grip High Performance Grout - Dayton Superior

Vibropruf \#11 - Lambert Corp.

\subsection{FABRICATION}

A. Shop Fabrication and Assembly: Fabricate and assemble structural assemblies in shop to greatest extent possible. Fabricate items of structural steel in accordance with AISC Specifications and as indicated on final shop drawings. Provide camber in structural members where indicated.

1. Properly mark and match-mark materials for field assembly. Fabricate for delivery sequence that will expedite erection and minimize field handling of materials.

2. Where finishing is required, complete assembly, including welding of units, before start of finishing operations. Provide finish surfaces of members exposed in final structure free of markings, burrs, and other defects.

B. Connections: Weld or bolt shop connections, as indicated.

1. Bolt field connections, except where welded connections or other connections are indicated.

2. Provide high-strength threaded fasteners, unless otherwise indicated.

C. High-Strength Bolted Connections: Install high-strength threaded fasteners in accordance with AISC "Specifications for Structural Joints using ASTM A325 or A490 Bolts". 
D. Welded Construction: Comply with AWS Code for procedures, appearance and quality of welds, and methods used in correcting welding work.

E. Shear Connectors: Prepare steel surfaces as recommended by manufacturer of shear connectors. Weld shear connectors in field, spaced as shown, to beams and girders in composite construction. Use automatic end welding of headed stud shear connectors in accordance with manufacturer's printed instructions.

F. Steel Wall Framing: Select members that are true and straight for fabrication of steel wall framing. Straighten as required to provide uniform, square, and true members in complete wall framing.

Build up welded door frames attached to structural steel framing. Weld exposed joints continuously and grind smooth. Plug-weld steel bar stops to frames, except where shown removable. Secure removable stops to frames with countersunk, cross-recessed head machine screws, uniformly spaced not more than 10 inches $(25 \mathrm{~cm})$ o.c., unless otherwise indicated.

G. Holes for Other Work: Provide holes required for securing other work to structural steel framing and for passage of other work through steel framing members, as shown on final shop drawings.

1. Provide threaded nuts welded to framing and other specialty items as indicated to receive other work.

2. Cut, drill, or punch holes perpendicular to metal surfaces. Do not flamecut holes or enlarge holes by burning. Drill holes in bearing plates.

\subsection{SHOP PAINTING}

A. General: Shop-paint structural steel, except those members or portions of members to be embedded in concrete or mortar. Paint embedded steel that is partially exposed on exposed portions and initial 2 inches $(51 \mathrm{~mm})$ of embedded areas only.

1. Do not paint surfaces to be welded or high-strength bolted with frictiontype connections.

2. Do not paint surfaces scheduled to receive sprayed-on fireproofing.

3. Apply 2 coats of paint to surfaces that are inaccessible after assembly or erection. Change color of second coat to distinguish it from first. 
B. Surface Preparation: After inspection and before shipping, clean steelwork to be painted. Remove loose rust, loose mill scale, and spatter, slag, or flux deposits. Clean steel in accordance with SSPC as follows:

$$
\begin{array}{ll}
\text { SP-1 } & \text { "Solvent Cleaning" } \\
\text { SP-2 } & \text { "Hand-Tool Cleaning" } \\
\text { SP-3 } & \text { "Power-Tool Cleaning" }
\end{array}
$$

C. Painting: Immediately after surface preparation, apply structural steel primer paint in accordance with manufacturer's instructions and at a rate to provide dry film thickness of not less than 3.0 mils. Use painting methods that result in full coverage of joints, corners, edges, and exposed surfaces.

\subsection{SOURCE QUALITY CONTROL}

Materials and fabrication procedures are subject to inspection and tests in mill, shop, and field, conducted by a qualified inspection agency. Such inspections and tests will not relieve Contractor of responsibility for providing materials and fabrication procedures in compliance with specified requirements. Promptly remove and replace materials or fabricated components that do not comply.

\section{PART 3 - EXECUTION}

\subsection{ERECTION}

A. Temporary Shoring and Bracing: Provide temporary shoring and bracing members with connections of sufficient strength to bear imposed loads. Remove temporary members and connections when permanent members are in place and final connections are made. Provide temporary guy lines to achieve proper alignment of structures as erection proceeds.

B. Anchor Bolts: Furnish anchor bolts and other connectors required for securing structural steel to foundations and other in-place work.

Furnish templates and other devices as necessary for presetting bolts and other anchors to accurate locations.

C. Setting Bases and Bearing Plates: Clean concrete and masonry bearing surfaces of bond-reducing materials and roughen to improve bond to surfaces. Clean bottom surface of base and bearing plates. 
1. Set loose and attached base plates. and bearing plates for structural members on wedges or other adjusting devices.

2. Tighten anchor bolts after supported members have been positioned and plumbed. Do not remove wedges or shims, but if protruding, cut off flush with edge of base or bearing plate prior to packing with grout.

3. Pack grout solidly between bearing surfaces and bases or plates to insure that no voids remain. Finish exposed surfaces, protect installed materials, and allow to cure.

4. For proprietary grout materials, comply with manufacturer's instructions.

D. Field Assembly: Set structural frames accurately to lines and elevations indicated and in accordance with AISC Specifications. Align and adjust various members forming part of complete frame or structure before permanently fastening. Clean bearing surfaces and other surfaces that will be in permanent contact before assembly. Perform necessary adjustments to compensate for discrepancies in elevations and alignment.

1. Level and plumb individual members of structures within specified AISC tolerances.

2. Establish required leveling and plumbing measurements on mean operating temperature of structure. Make allowances for difference between temperature at time of erection and mean temperature at which structure will be when completed and in service.

3. Splice members only where indicated and accepted on shop drawings.

4. Comply with AISC Specifications for bearing, adequacy of temporary connections, alignment, and removal of paint on surfaces adjacent to field welds.

5. Do not enlarge unfair holes in members by burning or by using drift pins, except in secondary bracing members. Ream holes that must be enlarged to admit bolts.

E. Gas Cutting: Do not use gas cutting torches in field for correcting fabrication errors in primary structural framing. Cutting will be permitted only on secondary members that are not under stress, as acceptable to the SDR. Finish gas-cut sections equal to a sheared appearance when permitted. Comply with NFPA $51 \mathrm{~B}$ for cutting processes. 
F. Touch-Up Painting: Immediately after erection, clean field welds, bolted connections, and abraded areas of shop paint. Apply paint to exposed areas using same material as used for shop painting.

Apply by brush or spray to provide minimum dry film thickness of 3.0 mils.

\subsection{QUALITY CONTROL}

A. Sandia National Laboratories (SNL) may engage an independent testing and inspection agency to inspect high-strength bolted connections and welded connections and to perform tests and prepare test reports.

1. Testing agency shall conduct and interpret tests, state in each report whether test specimens comply with requirements, and specifically state any deviations therefrom.

2. Provide access for testing agency to places where structural steel work is being fabricated or produced so required inspection and testing can be accomplished.

3. Testing agency may inspect structural steel at plant before shipment. SNL reserves the right, at any time before final acceptance, to reject materials not complying with specified requirements.

B. Correct Deficiencies in structural steel work that inspections and laboratory test reports have indicated are not in compliance with requirements. Perform additional tests, at Contractor's expense, as necessary to reconfirm any noncompliance of original work and to show compliance of corrected work.

C. Shop-Bolted and Field-Bolted Connections: Inspect or test in accordance with AISC Specifications.

Verify that gaps of installed direct tension indicators are less than gaps specified in ASTM F959, Table 2.

D. Shop Welding and Field Welding: Inspect and test during fabrication for shop welding and during erection for field welding, of structural steel assemblies, as follows:

1. Certify welders and conduct inspections and tests as required. Record types and locations of defects found in work. Record work required and performed to correct deficiencies.

2. Perform visual inspection of all welds. 
3. Perform tests up to and including $100 \%$ of welds at SNL's option. Inspection procedures may include the following:

a. Liquid Penetrant Inspection: ASTM E165.

b. Magnetic Particle Inspection: ASTM E709; performed on root pass and on finished weld. Cracks or zones of incomplete fusion or penetration are not acceptable.

c. Radiographic Inspection: ASTM E94 and ASTM E142; minimum quality level "2-2T".

d. Ultrasonic Inspection: ASTM E164.

4. Acceptance criteria shall be as specified in AWS D1.1.

END OF SECTION 05120 


\section{STANDARD SPECIFICATION . \\ SECTION 05500 \\ METAL FABRICATIONS}

\section{PART 1 - GENERAL}

\subsection{DESCRIPTION OF WORK}

A. This section includes the following metal fabrications:

Rough hardware

Ladders

Ladder safety cages

Loose bearing and leveling plates

Loose steel lintels

Miscellaneous framing, supports and trim

Shelf and ledger angles

Metal bar gratings

Floor plates

Tread plates

Steel pipe guardrails and handrails

Cast nosings and treads

Metal stairs

Pipe bollards

B. Related Sections: Refer to Division 5, Section "Structural Steel" for structural steel framing components.

\subsection{REFERENCES}

A. Aluminum Association (AA)

B. American National Standards Institute (ANSI)

A14.3 Safety Requirements for Fixed Ladders

C. American Society of Testing and Materials (ASTM)

A27 Specification for Steel Castings, Carbon, for General Application

A36 Specification for Structural Steel

A47 Specification for Ferritic Malleable Iron Castings

A48 Specification for Gray Iron Castings

A53 Specification for Pipe, Steel, Black and Hot-Dipped, Zinc-Coated Welded and Seamless 
A123 Specification for Zinc (Hot-Dip Galvanized) Coatings on Iron and Steel Products

A153 Specification for Zinc Coating (Hot-Dip) on Iron and Steel Hardware

A167 Specification for Stainless and Heat-Resisting Steel Bars and Shapes

A276 Specification for Stainless and Heat-Resisting Steel Bars and Shapes

A307 Specification for Carbon Steel Bolts and Studs, 60,000 PSI Tensile Strength

A366 Specification for Steel, Sheet, Carbon, Cold-Rolled, Commercial Quality

A446 Specification for Steel Sheet, Zinc-Coated (Galvanized) by the Hot-Dip Process, Structural (Physical) Quality

A500 Specification for Cold-Formed Welded and Seamless Carbon Steel Structural Tubing in Rounds and Shapes

A510 Specification for General Requirements for Wire Rods and Coarse Round Wire, Carbon Steel

A526 Specification for Steel Sheet, Zinc-Coated (Galvanized) by the Hot-Dip Process, Commercial Quality

A569 Specification for Steel, Carbon (0.15 Maximum, Percent), HotRolled Sheet and Strip, Commercial Quality

A570 Specification for Steel, Sheet and Strip, Carbon, Hot-Rolled, Structural Quality

A780 Practice for Repair of Damaged and Uncoated Areas of Hot-Dip Galvanized Coatings

A786 Specification for Rolled Steel Floor Plates

B221 Specification for Aluminum and Aluminum-Alloy Extruded Bars, Rods, Wire, Shapes, and Tubes

B632 Specification for Aluminum-Alloy Rolled Tread Plate

C1107 Specification for Packaged Dry, Hydraulic-Cement Grout (Nonshrink)

E985 Specification for Permanent Metal Railing Systems and Rails for Building

D. American Welding Society (AWS) 
D1.1 Structural Welding Code - Steel

D1.3 Structural Welding Code - Sheet Steel

E. Code of Federal Regulations (CFR)

Title 29 Part 1910 Labor-Occupational Safety and Health Standards

F. Corps of Engineers (CE)

CRD C621 Specification for Nonshrink Grout

G. Federal Specifications (FS)

FF-B-561 Bolts, Screw, Lag

FF-B-588 Bolt, Toggle, and Expansion Sleeve, Screw

FF-S-92 Screw, Machine, Slotted, Cross-Recessed or Hexagon Head

FF-S-111 Screw, Wood

FF-S-325 Shield, Expansion; Nail, Expansion; and Nail, Drive Screw (Devices, Anchoring, Masonry)

FF-W-84 Washers, Lock (Spring)

FF-W-92 Washer, Flat (Plain)

TT-P-664 Primer Coating, Alkyd, Corrosion-Inhibiting, Lead and Chromate Free, VOC-Compliant

TT-P-645 Primer, Paint, Zinc Chromate, Alkyd Type

H. Military Standardization Documents (MIL)

P-21035 Paint, High Zinc Dust Content, Galvanizing Repair

I. National Association of Architectural Metal Manufacturers (NAAMM)

AMP $500 \quad$ Metal Finishes Manual

AMP $510 \quad$ Metal Stair Manual

MBG 531 Metal Bar Grating Manual

MBG 532 Heavy Duty Metal.Bar Grating Manual

Pipe Railing Manual

J. National Fire Protection Association (NFPA)

101

Life Safety Code 
K. Steel Structures Painting Council (SSPC)

SSPC-PA 1 Paint Application Specification No. 1

SSPC Paint 12 Paint Specification No. 12 Cold Applied Asphalt Mastic (Extra Thick Film)

SSPC Paint 20 Paint Specification No. 20 Zinc-Rich Primers (Type I, "Inorganic," and Type II, "Organic")

SSPC-SP1 Surface Preparation Specification No. 1 "Solvent Cleaning"

SSPC-SP2 Surface Preparation Specification No. 2 "Hand-Tool Cleaning"

SSPC-SP3 Surface Preparation Specification No. 3 "Power Tool Cleaning"

SSPC-SP7 Surface Preparation Specification No. 7 "Brush-Off Blast Cleaning"

L. Uniform Building Code (UBC)

M. Uniform Federal Accessibility Standards (UFAS)

Fed. Std. 795

\subsection{DEFINITIONS}

A. Refer to ASTM E985 for railing-related terms that apply to this section.

B. Refer to the NAAMM publications listed in Article 1.02 "References" for definition of terms that apply to this section.

\subsection{SUBMITTALS}

General: Submit the following in accordance with Conditions of Contract and Division 1, Section "Descriptive Submittals".

A. Product Data: Submit product data for products used in metal fabrications, including paint products, grout and fasteners.

B. Shop Drawings: Submit detailed shop and erection drawings of each metal fabrication indicated. Include plans, elevations, sections, and details of metal fabrications and their connections. Show anchorage and accessory items.

C. Welder certificates signed by Contractor certifying that welders comply with requirements specified in $1.05 \mathrm{C}$.

D. Qualification data for firm specified in $1.05 \mathrm{~B}$ to demonstrate their capabilities and experience. 


\subsection{QUALITY ASSURANCE}

A. Codes and Standards: Comply with provisions of the following, except as otherwise indicated. Where conflicts occur, comply with the more stringent requirements.

1. ANSI 14.3

2. AWS D1.1 and D1.3

3. NFPA 101

B. Fabricator Qualifications: Firm experienced in successfully producing metal fabrications similar to that shown on the drawings, with sufficient production capacity to produce required units without causing delay in the work.

C. Qualify welding processes and welding operators in accordance with AWS D1.1 and D1.3.

Certify that each welder has satisfactorily passed AWS qualification tests for welding processes involved and, if pertinent, has undergone recertification.

D. All materials used shall be free of lead, and asbestos fibers.

E. Use of damaged items is prohibited except by specific authorization of Sandia Delegated Representative (SDR) in writing.

\subsection{DELIVERY. STORAGE. AND HANDLING}

A. Deliver materials to the job site in good condition and properly protected against damage to finished surfaces.

B. Storage on Site: Store materials in a location and in a manner to avoid damage. Stacking shall be done in a way which will prevent bending.

Store metal components and materials in a clean, dry location. Cover with waterproof paper, tarpaulin or polyethylene sheeting in a manner that will permit circulation of air inside the cover.

C. Keep handling on-site to a minimum. Exercise care to avoid damage to finishes of material.

\subsection{PROJECT CONDITIONS}

Field Measurements: Check actual locations of walls and other construction to which metal fabrications must fit, by accurate field measurements before fabrication; show recorded measurements on final shop drawings. Coordinate fabrication schedule with construction progress to avoid delay of work. 
Where field measurements cannot be made without delaying the work, guarantee dimensions and proceed with fabrication of products without field measurements. Coordinate construction to ensure that actual opening dimensions correspond to guaranteed dimensions. Allow for trimming and fitting.

\subsection{SEQUENCING AND SCHEDULING}

Sequence and coordinate installation of wall handrails as follows:

A. Mount handrails only on completed walls. Do not support handrails temporarily by any means not satisfying structural performance requirements.

B. Mount handrails only on gypsum board assemblies reinforced with proper blocking and backing to receive anchors, and where the location of concealed anchor plates has been clearly marked for benefit of Installer.

\section{PART 2 - PRODUCTS}

\subsection{FERROUS METALS}

A. Metal Surfaces, General: Form metal fabrications exposed to view upon completion of the work, provide materials selected for their surface flatness, smoothness, and freedom from surface blemishes. Do not use materials whose exposed surfaces exhibit pitting, seam marks, roller marks, rolled trade names, roughness, and, for steel sheet, variations in flatness exceeding those permitted by referenced standards for stretcher-leveled sheet.

B. Steel Plates. Shapes. and Bars: ASTM A36.

C. Rolled Steel Floor Plates: ASTM A786.

D. Steel Bars for Gratings: ASTM A569 or ASTM A36.

E. Wire Rod for Grating Cross Bars: ASTM A510.

F. Steel Tubing: Cold-formed, ASTM A500, Grade B, unless otherwise indicated.

G. Uncoated Structural Steel Sheet: Hot-rolled, ASTM A570, Grade 30 unless otherwise indicated.

H. Uncoated Steel Sheet (Commercial quality): Cold-rolled, ASTM A366.

I. Galvanized Steel Sheet: Quality as follows:

1. Structural Quality: ASTM A446; Grade A, unless another grade required for design loading, and G90 coating designation unless otherwise indicated. 
2. Commercial Quality: ASTM A526, G90 coating designation unless otherwise indicated.

J. Steel Pipe: ASTM A53, Type S, Grade B, standard weight (schedule 40), black finish, unless otherwise indicated.

K. Gray Iron Castings: ASTM A48, Class 30.

L. Malleable Iron Castings: ASTM A47, Grade 32510.

M. Brackets. Flanges and Anchors: Cast or formed metal of the same type material and finish as supported rails, unless otherwise indicated.

N. Concrete Inserts: Threaded or wedge type; galvanized ferrous castings, either malleable iron, ASTM A47, or cast steel, ASTM A27. Provide bolts, washers, and shims as required, hot-dip galvanized per ASTM A153.

O. Welding Rods and Bare Electrodes: Select in accordance with AWS specifications for the metal alloy to be welded.

\subsection{STAINLESS STEEL}

A. Bar Stock: ASTM A276, Type 302 or 304.

B. Plate: ASTM A167, Type 302 or 304.

2.03 Aluminum

A. Extruded Bars and Shapes: ASTM B221, alloy 6061-T6 or 6063-T6 for bearing bars of gratings and shapes and 6061-T6 for grating cross bars.

B. Aluminum-Alloy Rolled Tread Plate: ASTM B632, alloy 6061-T4 for treads and 6061-T6 for platforms.

\subsection{GROUT}

Nonshrink Nonmetallic Grout: Premixed, factory-packaged, nonstaining, noncorrosive, nongaseous grout complying with ASTM C1107 (formerly referenced as CE CRD C621). Provide grout specifically recommended by manufacturer for interior and exterior applications of type specified in this section.

Subject to compliance with requirements, products that may be incorporated in the work include, but are not limited to the following:

Euco N-S Grout - Euclid Chemical Co.

Kemset - Chem-Masters Corp.

Crystex - L \& M Construction Chemicals, Inc:

Sonogrout - Sonneborn Building Products Div., Rexnord Chemical Products, Inc.

Supreme Grout - Cormix, Inc.

Sure-Grip High Performance Grout - Dayton Superior 
Vibropruf \#11 - Lambert Corp.

\subsection{FASTENERS}

A. General: Provide zinc-coated fasteners for exterior use or where built into exterior walls. Select fasteners for the type, grade, and class required. Suspect/counterfeit bolts will not be accepted and will be replaced at Contractor's expense.

B. Bolts and Nuts: Regular hexagon head type, ASTM A307, Grade A.

C. Lag Bolts: Square head type, FS FF-B-561.

D. Machine Screws: FS FF-S-92.

E. Wood Screws: Flat head carbon steel, FS FF-S-111.

F. Plain Washers: Round, carbon steel, FS FF-W-92.

G. Expansion Anchors: Expansion anchors complying with FS FF-S-325.

H. Toggle Bolts: Tumble-wing type, FS FF-B-588, type, class, and style as required.

I. Lock Washers: Helical spring type carbon steel, FS FF-W-84.

J. Special Fasteners: Split nuts, indicator washers and others, as indicated.

2.06 PAINT

A. Shop Primer for Ferrous Metal: Red oxide, lead- and cadmium-free, corrosion-inhibiting primer complying with performance requirements of $F S$ TT-P-664.

B. Galvanizing Repair Paint: High zinc dust content paint for regalvanizing welds in galvanized steel, with dry film containing not less than $94 \%$ zinc dust by weight, and complying with MIL-P-21035 or SSPC-Paint-20.

C. Bituminous Paint: Cold-applied asphalt mastic complying with SSPCPaint-12 except containing no asbestos fibers.

D. Zinc Chromate Primer: FS TT-P-645.

\subsection{CONCRETE FILL}

A. Concrete Materials and Properties: Comply with requirements for Division 3, Section "Cast-in-Place Concrete" for normal weight, ready-mix concrete with minimum 28-day compressive strength of 3000 psi (21 MPa), unless higher strength indicated. 
B. Nonslip Aggregate Finish: Factory-graded, packaged material containing fused aluminum oxide grits or crushed emery as abrasive aggregate; rustproof and nonglazing; unaffected by freezing, moisture, or cleaning materials.

\subsection{FABRICATION. GENERAL}

A. Form metal fabrications from materials of size, thickness, and shapes indicated but not less than that needed to comply with performance requirements indicated. Work to dimensions indicated or accepted on shop drawings, using proven details of fabrication and support. Use type of materials indicated or specified for various components of each metal fabrication.

B. Form exposed work true to line and level with accurate angles and surfaces and straight sharp edges.

C. Allow for thermal movement resulting from the following maximum change (range) in ambient temperature in the design, fabrication, and installation of installed metal assemblies to prevent buckling, opening up of joints, and overstressing of welds and fasteners. Base design calculations on actual surface temperatures of metals due to both solar heat gain and nighttime sky heat loss.

Temperature change (Range): $100 \operatorname{deg} \mathrm{F}(38 \mathrm{deg} \mathrm{C})$.

D. Shear and punch metals cleanly and accurately. Remove burrs.

E. Ease exposed edges to a radius of approximately $1 / 32$ inch $(0.794 \mathrm{~mm})$, unless otherwise indicated. Form bent-metal corners to smallest radius possible without causing grain separation or otherwise impairing work.

F. Remove sharp or rough areas on exposed traffic surfaces.

G. Weld corners and seams continuously to comply with AWS recommendations and the following:

1. Use materials and methods that minimize distortion and develop strength and corrosion resistance of base metals.

2. Obtain fusion without undercut or overlap.

3. Remove welding flux immediately.

4. At exposed connections, finish exposed welds and surfaces smooth and blended so that no roughness shows after finishing and contour of welded surface matched those adjacent.

H. Form exposed connections with hairline joints, flush and smooth, using concealed fasteners wherever possible. Use exposed fasteners of type indicated or, if not indicated, Phillips flat-head (countersunk) screws or bolts. Locate joints where least conspicuous. 
I. Provide for anchorage of type indicated; coordinate with supporting structure. Fabricate and space anchoring devices to provide adequate support for intended use.

J. Shop Assembly: Preassemble items in shop to greatest extent possible to minimize field splicing and assembly. Disassemble units only as necessary for shipping and handling limitations. Use connections that maintain structural value of joined pieces. Clearly mark units for reassembly and coordinated installation.

K. Cut reinforce, drill and tap miscellaneous metal work as indicated to receive finish hardware, screws, and similar items.

L. Fabricate joints that will be exposed to weather in a manner to exclude water, or provide weep holes where water may accumulate.

\subsection{ROUGH HARDWARE}

Furnish bent or otherwise custom fabricated bolts, plates, anchors, hangers, dowels, and other miscellaneous steel and iron shapes as required. Fabricate items to sizes, shapes, and dimensions required.

\subsection{STEEL LADDERS}

A. General: Fabricate ladders for the locations shown, with dimensions, spacings, details and anchorages as indicated. Comply with requirements of ANSI A14.3 and CFR 29 1910.27; where conflicts occur, comply with the more stringent requirements.

B. Side Rails: Continuous steel flat bars, minimum size (cross section) 2-1/2" x 3/8" (63.5 mm x $9.5 \mathrm{~mm})$, with eased edges, spaced 18 inches $(0.46 \mathrm{~m})$ apart.

For ladders subject to unusually corrosive atmospheric exposures, steel flat bars $2-1 / 2 " \times 1 / 2 "(63.5 \mathrm{~mm} \times 12.7 \mathrm{~mm})$.

C. Bar Rungs: Round steel bars, minimum $3 / 4$ inch $(19.1 \mathrm{~mm})$ diameter, spaced no greater than 12 inches $(305 \mathrm{~mm}$ ) o.c. and uniform throughout the length of the ladder.

Individual metal rungs embedded in concrete which serve as access to pits and other areas under floors, should have a minimum diameter of 1 inch $(25 \mathrm{~mm})$ or shall otherwise be treated to resist corrosion and rusting.

D. Fit rungs in centerline of side rails, plug weld and grind smooth on outer rail faces.

E. Support each ladder at top and bottom and at intermediate points spaced not more than $5^{\prime}-0^{\prime \prime}(1.5 \mathrm{~m})$ o.c. by means of welded brackets, unless otherwise indicated. 
1. Size brackets to support design dead and live loads indicated and to hold centerline of ladder rungs clear of the wall surface by not less than 7 inches $(178 \mathrm{~mm})$.

2. Extend side rails 42 inches $(1.1 \mathrm{~m})$ above top rung, and return rails to wall or structure unless other secure handholds are provided. If the adjacent structure does not extend above the top rung, goose-neck the extended rails back to the structure to provide secure ladder access.

\subsection{LADDER SAFETY CAGES}

A. General: Fabricate ladder safety cages to comply with requirements of ANSI A14.3 and CFR 29 1910.27; where conflicts occur, comply with the more stringent requirements. Assemble safety cages by welding.

B. Primary Hoops: Steel bars, 3" x 1/4" (76 mm x $6.4 \mathrm{~mm})$, for top, bottom, and for cages longer than 20 feet $(6.1 \mathrm{~m})$, intermediate hoops spaced not more than $20^{\prime}-0^{\prime \prime}(6.1 \mathrm{~m})$ o.c.

C. Secondary Intermediate Hoops: Steel bars, 2" x 1/4" (51 mm x $6.4 \mathrm{~mm})$ hoops spaced not more than 4'-0" $(1.2 \mathrm{~m})$ o.c. between primary hoops.

D. Vertical Bars: Steel bars, 1-1/2" x 1/4" (38.1 mm x $6.4 \mathrm{~m})$, secured to each hoop, spaced at intervals not more than $40^{\circ}(0.7 \mathrm{rad})$ o.c. around the circumference of the cage, maximum spacing of approximately 9-1/2 inches $(241 \mathrm{~mm})$ o.c.

E. Fasten assembled safety cage to ladder rails and adjacent construction as indicated.

\subsection{LOOSE BEARING AND LEVELING PLATES}

Provide loose bearing and leveling plates for steel items bearing on masonry or concrete construction, made flat, free from warps or twists, and of required thickness and bearing area. Drill plates to receive anchor bolts and for grouting as required.

\subsection{LOOSE STEEL LINTELS}

A. Fabricate loose structural steel lintels from steel angles and shapes of size indicated for opening and recesses in masonry walls and partitions at locations indicated.

B. Weld adioining members together to form a single unit where indicated.

C. Size loose lintels for equal bearing of one inch per foot of clear span but not less than 8 inches $(203 \mathrm{~mm}$ ) bearing at each side of openings, unless otherwise indicated.

\subsection{MISCELLANEOUS METAL ITEMS}


A. Miscellaneous Framing and Supports: Provide steel framing and supports for applications indicated which are not a part of structural steel framework, as required to complete work.

1. Fabricate units to sizes, shapes, and profiles indicated and required to receive adjacent other construction retained by framing and supports. Fabricate from structural steel shapes, plates, and steel bars of welded construction using mitered joints for field connection. Cut, drill, and tap units to receive hardware, hangers, and similar items.

2. Equip units with integrally welded anchors for casting into concrete or building into masonry. Furnish inserts if units must be installed after concrete is placed.

Except as otherwise indicated, space anchors 24 inches $(61 \mathrm{~cm})$ o.c. and provide minimum anchor units in the form of steel straps 1-1/4" wide $\times 1 / 4$ " $\times 8$ " long (31.8 $\mathrm{mm} \times 6.4 \mathrm{~mm}$ x $203 \mathrm{~mm}$ ).

B. Miscellaneous Steel Trim: Provide shapes and sizes indicated for profiles shown. Unless otherwise indicated, fabricate units from structural steel shapes, plates, and steel bars, with continuously welded joints and smooth exposed edges. Use concealed field splices wherever possible. Provide cutouts, fittings, and anchorages as required for coordination for assembly and installation with other work.

\subsection{SHELF AND LEDGER ANGLES}

A. Fabricate shelf and ledger angles from steel angles of sizes indicated and for attachment to concrete framing. Provide slotted holes to receive $3 / 4$ inch $(19.1 \mathrm{~mm})$ bolts, spaced not more than 6 inches $(152 \mathrm{~mm})$ from ends and not more than 24 inches $(0.61 \mathrm{~m})$ o.c., unless otherwise indicated.

B. For cavity walls, provide vertical channel brackets to support shelf/ledger angles from back-up masonry and concrete. Align expansion joints in angles with indicated expansion joints in cavity wall exterior wythe.

C. Furnish wedge-type concrete inserts, complete with fasteners, for attachment of shelf angles to cast-in-place concrete.

\subsection{METAL BAR GRATINGS}

A. General: Produce metal bar gratings of description indicated per NAAMM marking system that comply with the following:

1. Metal Bar Grating Standard: "Standard Specifications for Metal Bar Grating and Metal Bar Grating Treads" published in ANSI/NAAMM MBG 531.

2. Heavy Duty Metal Bar Grating Standard: "Guide Specifications for Heavy Duty Metal Bar Grating" published in ANSI/NAAMM -MBG 532. 
B. If gratings are located in walking surfaces, gratings shall have spaces no greater than $1 / 2$ inch $(12.7 \mathrm{~mm})$ wide in one direction.

If gratings have elongated openings, then they shall be placed so that the long dimension is perpendicular to the dominant direction of travel.

C. Fabricate steel gratings to comply with requirements as indicated on the drawings.

D. Traffic Surface for Steel and Aluminum Bar Gratings: Plain, unless otherwise indicated.

E. Steel Finish: Shop prime paint applied in accordance with manufacturer's standard practice.

F. Aluminum Finish: Mill (as fabricated), unless otherwise indicated.

G. Fabricate removable grating sections with banding bars attached by welding to entire perimeter of each section. Include anchors and fasteners of type indicated, or if not indicated, as recommended by manufacturer, for attachment to supports.

1. Heavy Duty Grating: Provide not less than 4 anchor blocks, $1 / 4$ inch $(6.4 \mathrm{~mm})$ minimum thickness, for each section of grating composed of bearing bars over $3 / 16$ inch $(4.8 \mathrm{~mm})$ in thickness, with each block shopwelded to 2 bearing bars.

2. Nonheavy Duty Grating: Provide not less than 4 saddle clips for each grating section composed of rectangular bearing bars $3 / 16$ inch $(4.8 \mathrm{~mm})$ or less in thickness and spaced not less than $15 / 16$ inch $(23.8 \mathrm{~mm})$ o.c., with each clip designed and fabricated to fit over 2 bearing bars.

Furnish threaded bolts with nuts and washers for each clip required, unless otherwise indicated.

H. Attach toe plates to grating by welding, unless otherwise indicated.

Toe plate height: 4 inches $(102 \mathrm{~mm})$, unless a greater height indicated.

I. Fabricate cutouts in grating sections for penetrations indicated. Arrange layout of cutouts to permit grating removal without disturbing items penetrating gratings.

1. Edge band openings in grating that interrupt 4 or more bearing bars with bars of same size and material as bearing bars.

2. Do not notch bearing bars at supports to maintain elevation.

J. Available Manufacturers: Subject to compliance with requirements, manufacturers offering metal bar gratings that may be incorporated in the Work include, but are not limited to, the following:

Alabama Metal Industries Corp.

Barnet/Bates Corp. 
IKG Industries

Klemp Corp.

Ohio Gratings, Inc.

Seidelhuber Metal Products, Inc.

\subsection{FLOOR PLATE}

A. Fabricate raised pattern floor plates from rolled steel floor plate of thickness and pattern indicated. If not indicated, pattern as selected from manufacturer's standard patterns. See ASTM A786 for pattern illustrations.

B. Abrasive Surface Floor Plate: Manufacturer's standard abrasive granules, rolled into surface of steel plate, where indicated.

C. Include steel angle stiffeners and fixed and removable sections as indicated.

Provide 2 steel bar drop handles for lifting plates, one at each end of each removable section.

\subsection{TREAD PLATE}

A. Fabricate raised pattern tread plates from aluminum-alloy rolled tread plate in pattern and thickness as indicated. See ASTM B632 for pattern illustrations.

B. Abrasive Surface Floor Plate: Manufacturer's standard abrasive granules, rolled into surface of aluminum plate, where indicated.

C. Include aluminum angle stiffeners and fixed and removable sections as indicated.

Provide 2 aluminum bar drop handles for lifting plates, one at each end of each removable section.

\subsection{STEEL PIPE GUARDRAILS AND HANDRAILS}

A. General: Fabricate pipe guardrails and handrails to comply with requirements indicated for dimensions, details, finish, and member sizes, including wall thickness of pipe, post spacings, and anchorage.

B. Interconnect guardrails and handrail members by butt-welding or welding with internal connectors, at fabricator's option, unless otherwise indicated.

At tee and cross intersections, cope ends of intersecting members to fit contour of pipe to which end is joined, weld all around and grind smooth.

C. Form changes in directions of railing members as follows:

1. By use of welded prefabricated steel elbow fittings. 
2. By bending, of radius indicated.

3. By mitering at elbow bends.

D. Form simple and compound curves by bending pipe in jigs to produce uniform curvature for each repetitive configuration required; maintain cylindrical cross-section of pipe throughout entire bend without buckling, twisting, cracking, or otherwise deforming exposed surfaces of pipe.

E. Provide wall returns at ends of wall-mounted handrails, unless otherwise indicated.

F. Close exposed ends of pipe by welding $3 / 16$ inch $(4.8 \mathrm{~mm})$ thick steel plate in place or by use of prefabricated fittings, except where clearance of end of pipe and adjoining wall surface is $1 / 4$ inch $(6.4 \mathrm{~mm})$ or less.

G. Toe Boards: Where indicated, provide toe boards at railings around openings and at the edge of open-sided floors and platforms. Fabricate to dimensions and details indicated, or if not indicated, use 4 inches high $\times 1 / 4$ inch (102 mm x $6.4 \mathrm{~mm}$ ) steel bar welded to each railing post.

H. Brackets. Flanges. Fittings, and Anchors: Provide wall brackets, end closures, flanges, miscellaneous fittings, and anchors for interconnections of pipe and attachment of guardrails and handrails to other work. Furnish inserts and other anchorage devices for connecting guardrails and handrails to concrete or masonry work.

1. For railing posts set in concrete, fabricate sleeves from steel pipe not less than 6 inches $(152 \mathrm{~mm})$ long and with an inside diameter not less than $1 / 2$ inch $(12.7 \mathrm{~mm})$ greater than the outside diameter of post, with steel plate closure welded to bottom of sleeve.

2. For removable railing posts, fabricate slip-fit sockets from steel pipe whose inside diameter is sized for a close fit with posts and to limit deflection of post without lateral load, measured at top, to not more than 1/12 of post height. Provide socket covers designed and fabricated to resist accidental dislodgement.

I. Fillers: Provide steel sheet or plate fillers of thickness and size indicated or required to support structural loads of handrails where needed to transfer wall bracket loads through wall finishes to structural supports. Size fillers to suit wall finish thicknesses. Size fills to produce adequate bearing to prevent bracket rotation and overstressing of substrate.

A. Fabricate units of material, sizes, and configurations indicated. If not indicated, provide cast-iron units with integral abrasive finish. Furnish in lengths as required to accurately fit each opening or conditions.

Cast units with an integral abrasive grit consisting of aluminum oxide, silicone carbide, or a combination of both. 
B. Available Manufacturers: Subject to compliance with requirements, manufacturers offering products that may be incorporated in the Work include, but are not limited to, the following:

AMSTEP Products, division of American Safety Technologies, Inc.

American Safety Tread Co., Inc.

IKG Industries

Safe-T-Metal Co., Inc.

Wooster Products Inc.

C. Provide anchors for embedding units in concrete, either integral or applied to units, as standard with the manufacturer.

D. Apply black asphaltic coating to concealed bottoms, sides, and edges of cast-iron units set into concrete.

\subsection{STEEL FRAMED STAIRS}

A. General: Construct stairs to conform to sizes and arrangements indicated with welded connections, unless otherwise indicated. Provide complete stair assemblies, including metal framing, hangers, columns, railings, newels, balusters, struts, clips, brackets, bearing plates, and other components necessary for the support of stairs and platforms, and as required to anchor and contain the stairs on the supporting structure.

Fabricate treads and platforms of exterior stairs to accommodate slopes to drain in finished traffic surfaces.

B. Stair Framing: Fabricate stringers of structural steel channels, or plates, or a combination thereof, as indicated. Provide closures for exposed ends of stringers. Construct platforms of structural steel channel headers and miscellaneous framing members as indicated. Bolt or weld headers to stringers, newels, and framing members to stringers and headers; fabricate and join so that bolts, if used, do not appear on finish surfaces.

C. Metal Pan Risers. Subtreads, and Subplatforms: Shape metal pans for risers and subtreads to conform to configuration shown. Provide thickness of structural steel sheet for metal pans as indicated.

1. Form metal pans of uncoated cold-rolled steel sheet, unless otherwise indicated.

2. Form metal pans of galvanized steel sheet, where indicated on the drawings.

3. Attach Risers and Subtreads to Stringers by the following methods:

a. Directly weld risers and subtreads to stringers; locate welds on side of metal pans to be concealed by concrete fill.

b. Attach by means of brackets made of steel angles or bars. Weld brackets to stringers and attach metal pans to brackets by welding or bolting. 
4. Provide subplatforms of configuration and construction shown, with thickness of structural steel sheet as indicated. Attach subplatform to platform framing members with welds or as otherwise indicated.

Construct subplatforms with smooth soffits.

D. Steel Floor Plate Treads and Platforms: Provide raised pattern steel floor plate in pattern indicated or, if not indicated, as selected from manufacturer's standard patterns.

1. Form treads of $1 / 4$ inch $(6.4 \mathrm{~mm})$ thick raised pattern steel floor plate with integral nosing and back edge stiffener. Weld steel supporting brackets to stringers and treads to brackets.

2. Fabricate platforms of raised pattern steel floor plate of thickness indicated. Provide nosing matching that on treads at all landings. Secure to platform framing members with welds.

E. Floor Grating Treads and Platforms: Provide patterns, spacing, and bar sizes indicated; fabricate to comply with NAAMM MBG 531 .

1. Finish to be shop prime paint, unless otherwise indicated.

2. Fabricate grating treads with steel plate nosing on one edge and with steel angle or steel plate carrier at each end for stringer connections. Secure treads to stringers with bolts or as otherwise indicated.

3. Fabricate grating platforms, with nosing matching that on grating treads, at all landings. Provide toe plates at open-sided edges of grating platform. Secure grating to platform frame with welds or as otherwise indicated.

F. Stair Guardrails and Handrails: Comply with applicable requirements specified elsewhere in this section for steel pipe guardrails and handrails, and as follows:

1. Fabricate newels of steel tubing and provide newel caps of gray-iron castings, as shown.

2. Railings may be bent at corners, rail returns, and wall returns, instead of using prefabricated fittings.

3. Connect railing posts to stair framing by direct welding, unless otherwise indicated.

\subsection{PIPE BOLLARDS}

Fabricate pipe bollards from 4-inch (102 mm) standard black steel pipe, Schedule 40 , unless otherwise indicated. Cap bollards with $1 / 4$ inch $(6.4 \mathrm{~mm})$ minimum thickness steel base plate, or as otherwise indicated.

\subsection{FINISHES}



A. General: Comply with NAAMM AMP 500 "Metal Finishes Manual" for recommendations relative to application and designations of finishes.
B. Finish metal fabrications after assembly.

\subsection{STEEL AND IRON FINISHES}

A. General: Shop-paint uncoated surfaces of metal fabrications, except those to be embedded in concrete or masonry or to receive sprayed-on fireproofing, surfaces and edges to be welded, and galvanized surfaces, unless otherwise indicated. Comply with requirements of SSPC-PA 1 for shop painting.

B. Galvanizing: For those items indicated for galvanizing, apply zinc-coating by the hot-dip process in compliance with the following requirements:

1. ASTM A123 for galvanizing both fabricated and unfabricated iron and steel products made of uncoated rolled, pressed, and forced shapes, plates, bars, and strip 0.0299 inch $(0.7595 \mathrm{~mm})$ thick and heavier.

2. ASTMA153 for galvanizing iron and steel hardware.

C. Surface Preparation for Shop Priming: Prepare uncoated ferrous metal surfaces to comply with minimum requirements indicated below.

1. Remove oil, grease and similar contaminants in accordance with SP-1, "Solvent Cleaning".

2. Remove loose rust, scale, spatter, slag and other deleterious materials in accordance with SSPC, utilizing the following methods as required:

SP-2 "Hand-Tool Cleaning"

SP-3 "Power-Tool Cleaning"

SP-7 "Brush-Off Blast Cleaning"

D. Painting: Immediately after surface preparation, apply structural steel primer paint in accordance with manufacturer's instructions and at a rate to provide dry film thickness of not less than 3.0 mils $(0.076 \mathrm{~mm})$. Use painting methods that result in full coverage of joints, corners, edges, and exposed surfaces.

Apply 2 coats of paint to surfaces that are inaccessible after assembly or erection.

\subsection{ALUMINUM FINISH}

Mill (as fabricated) finish, unless otherwise indicated.

\section{PART 3 - EXECUTION}

\subsection{1 examination}


Installer shall examine the areas and conditions under which metal fabrication items are to be installed. Notify the SDR in writing of conditions detrimental to the proper and timely completion of the work. Do not proceed with the work until unsatisfactory conditions have been corrected in a manner acceptable to the Installer and SDR.

A. Coordinate and furnish anchorages, setting drawings, diagrams, templates, instructions, and directions for installation of anchorages, including concrete inserts, sleeves, anchor bolts, and miscellaneous items having integral anchors that are to be embedded in concrete or masonry construction. Coordinate delivery of such items to project site.

B. Center nosings on tread widths with noses flush with riser faces and tread surfaces.

C. Set sleeves in concrete with tops flush with finish surface elevations; protect sleeves from water and concrete entry.

\subsection{INSTALLATION. GENERAL}

A. Fastening to In-Place Construction: Provide anchorage devices and fasteners where necessary for securing miscellaneous metal fabrications to in-place construction; include threaded fasteners for concrete and masonry inserts, toggle bolts, through-bolts, lag bolts, wood screws and other connectors as required.

B. Cutting. Fitting and Placement: Perform cutting, drilling, and fitting required for installation of miscellaneous metal fabrications. Set metal fabrication accurately in location, alignment, and elevation; with edges and surfaces level, plumb, true, and free of rack; and measured from established lines and levels.

C. Provide temporary bracing or anchors in formwork for items that are to be built into concrete masonry or similar construction.

D. Fit exposed connections accurately together to form hairline joints. Weld connections that are not to be left as exposed joints, but cannot be shopwelded because of shipping size limitations. Do not weld, cut, or abrade the surfaces of exterior units which have been hot-dip galvanized after fabrication, and are intended for bolted or screwed field connections.

E. Field Welding: Comply with AWS Code for procedures of manual shielded metal-arch welding, appearance and quality of welds made, methods used in correcting welding work, and the following:

1. Use materials and methods that minimize distortion and develop strength and corrosion resistance of base metals.

2. Obtain fusion without undercut or overlap. 
3. Remove welding flux immediately.

4. At exposed connections, finish exposed welds and surfaces smooth and blended so that no roughness shows after finishing and contour of welded surfaces matches those adjacent.

F. Grout: Follow manufacturer's recommendations for substrate preparation and application.

G. Corrosion Protection: Coat concealed surfaces of aluminum that will come into contact with grout, concrete, masonry, wood, or dissimilar metals with a heavy coat of bituminous paint or zinc chromate primer.

\subsection{SETTING LOOSE PLATES}

A. Clean concrete and masonry bearing surfaces of any bond-reducing materials, and roughen to improve bond to surfaces. Clean bottom surface of bearing plates.

B. Set loose leveling and bearing plates on wedges, or other adjustable devices. After the bearing members have been positioned and plumbed, tighten the anchor bolts. Do not remove wedges or shims, but if protruding, cut off flush with the edge of the bearing plate before packing with grout. Pack grout solidly between bearing surfaces and plates to ensure that no voids remain.

\subsection{INSTALLATION OF METAL BAR GRATINGS}

A. General: Install gratings to comply with requirements of NAAMM grating standard that apply to grating types and bar sizes indicated, including installation clearances and standard anchoring details.

B. Secure removable units to supporting members with type and size of clips and fasteners indicated, or if not indicated, as recommended by grating manufacturer for type of installation conditions shown.

C. Secure non-removable units to supporting members by welding where both materials are the same; otherwise, fasten by bolting as indicated above.

D. Attach toe plates to gratings by welding, at locations indicated.

\subsection{INSTALLATION OF STEEL PIPE GUARDRAILS AND HANDRAILS}

A. Adiust railings prior to anchoring to ensure matching alignment at abutting joints. Space posts at spacing indicated, or if not indicated, as required by design loadings. Plumb posts in each direction. Secure posts and railing ends to building construction as follows:

1. Anchor posts in concrete by means of pipe sleeves preset and anchored into concrete. After posts have been inserted into sleeves, fill annular space between post and sleeve solid with nonshrink, nonmetallic grout, 
mixed and placed to comply with anchoring material manufacturer's directions.

2. Anchor posts and rail ends to steel with welded connections, unless otherwise indicated.

3. Anchor posts and rail ends into concrete and masonry with steel round flanges welded to post and rail ends, and anchored into wall construction with expansion shields and bolts.

4. Install removable railing sections where indicated in slip-fit metal sockets cast into concrete. Accurately locate sockets to match post spacing.

B. Secure handrails to wall with wall brackets and end fittings. Provide bracket with not less than 1-1/2 inch $(38.1 \mathrm{~mm})$ clearance from inside face of handrail and finished wall surface. Locate brackets at spacing not less than $5^{\prime}-0^{\prime \prime}\left(1.5^{\prime} \mathrm{m}\right)$ o.c., unless otherwise indicated. Secure wall brackets and wall return fittings to building construction as follows:

1. Use type of bracket with flange tapped for concealed anchorage to threaded hanger bolt.

2. For concrete and solid masonry anchorage, use drilled-in expansion shield and either concealed hanger bolt or exposed lag bolt, as applicable.

3. For hollow masonry anchorage, fasten brackets directly on masonry wall using toggle bolts.

4. For steel framed gypsum board assemblies, fasten brackets to wood blocking using lag bolts or to metal blocking using self-tapping screws, of size and type required to support structural loads.

C. Expansion Joints: Provide expansion joints at locations indicated, or if not indicated, at intervals not to exceed 40 feet $(12.2 \mathrm{~m})$. Provide slip joint with internal sleeve extending 2 inches $(51 \mathrm{~mm})$ beyond joint on either side; fasten internal sleeve securely to one side; locate joint within 6 inches (152 $\mathrm{mm}$ ) of posts.

\subsection{INSTALLATION OF CAST NOSINGS AND TREADS}

Install cast nosings and treads with anchorage system indicated to comply with manufacturer's recommendations.

\subsection{INSTALLATION OF PIPE BOLLARDS}

Install bollards at locations shown on drawings. After installation, fill pipe with concrete and provide a smooth convex curve at the top of the pipe.

\subsection{ADJUSTING AND CLEANING}


A. Touch-Up Painting of Steel Items: Immediately after erection, clean field welds, bolted connections, abraded areas of shop paint, and paint exposed areas with same material as used for shop painting to comply with SSPC-PA 1 requirements for touch-up of field painted surfaces.

Apply by brush or spray to provide a minimum dry film thickness of 3.0 mils $(0.076 \mathrm{~mm})$.

B. For galvanized surfaces clean welds, bolted connections and abraded areas and apply galvanizing repair paint to comply with ASTM A780.

END OF SECTION 05500 


\section{STANDARD SPECIFICATION \\ SECTION 09900 \\ PAINTING}

\section{PART 1 - GENERAL}

\subsection{DESCRIPTION OF WORK}

A. Definition: "Paint" as used herein means all coating systems materials, including primers, emulsions, enamels, stains, sealers ant fillers, and other applied materials whether used as prime, intermediate, or finish coats. Surfaces listed in the painting schedule herein shall receive the surface preparation and number of coats prescribed.

B. Included: All exposed new work shall be painted unless excluded in Paragraph $1.01 \mathrm{C}$. or otherwise excluded. Existing work shall be painted when specified. Painting shall include:

1. Priming of damaged or abraded shop coats.

2. Those surfaces damaged and repaired by work connected with this project shall be painted unless the entire surface is otherwise shown or specified to be painted. Patch painting shall closely match the existing adjacent paint.

3. Color coding of sprinkler piping.

4. Color coding of all other Mechanical Piping Systems is limited to those items indicated in the contract documents.

5. Used full height metal partitions and patrol height metal cubicle partitions installed or relocated on this project.

6. Touch-up painting of all factory finishes damaged in shipment or installation and repainting if colors are not satisfactory.

C. Excluded: The following items shall not be painted except where otherwise noted on the drawings or in the contract documents:

1. Plated or factory-finished items, except where "factory finish" is prime coat only and except where colors are not satisfactory.

2. Roof flashing materials around roof-mounted equipment.

3. Finished metal surfaces including: Anodized aluminum, stainless steel, chromium plate, brass, and bronze. 
4. Glass.

5. Prefinished new acoustical materials.

6. Galvanized components of prefabricated metal buildings.

7. Interior and exterior concrete slabs on grade.

8. Equipment room surfaces.

\subsection{RELATED WORK}

Related work which is specified in other sections includes::

- Shop coats on fabricated items.

- Identification labels for piping.

- Taping and texturing of gypsum wallboard.

1.03 QUALITY ASSURANCE- Single Source Responsibility: Provide primers and other undercoat paint produced by same manufacturer as finish coats. Use only thinners approved by paint manufacturer, and use only within recommended limits.

\subsection{DELIVERY AND STORAGE}

A. Deliver materials to job site in original, new and unopened packages and containers bearing manufacturer's name and label, containing the following information:

- Name or title of material

- Manufacturer's stock number and date of manufacture

- Manufacturer's name

- Thinning instructions

- Application Instructions

- Color name and number

B. Store materials not in actual use in tightly covered containers. Maintain containers used in storage of paint in a clean condition, free of foreign materials and residue.

Protect from freezing where necessary. Keep storage area neat and orderly. Remove oily rags and waste daily. Take all precautions to ensure that 
workmen and work areas are adequately protected from fire hazards and health hazards resulting from handling, mixing and application of paints.

PART 2-PRODUCTS

2.01 MATERIALS - The federal specifications listed below establish minimum acceptable quality for paint materials. Provide written certification from paint manufacturer that materials provided meet or exceed these minimums.

TT-P-19 Paint, acrylic emulsion, exterior.

TT-P-25 Primer coating, exterior, undercoat for wood, ready-mixed, white and tints.

TT-P-29 Paint, latex base, interior, flat, white and tints.

TT-P-86 Paint, red-lead base, ready mixed.

TT-V-86 Varnish, oil, rubbing (satin finish).

TT-E-489 Enamel, alkyd, gloss, for exterior and interior surfaces, Type I (bulk), Class A (air-drying).

TT-E-509 Enamel, alkyd, semi-gloss, interior, odorless, white or tints.

TT-E-543 Enamel, undercoat, interior, tints and white.

TT-P-636 Primer coating, alkyd, wood and ferrous metal.

TT-P-641 Primer coating, zinc dust-zinc oxide.

TT-P-650 Primer coating, latex base, interior, white.

TT-P-1511 Paint, latex, interior, tints and white, Type I (Semigloss).

\subsection{MISCELLANEOUS MATERIALS}

A. Fire-retardant paint shall be a coating that is U.L. listed as having a flamespread and smoke-developed rating of 25 or less. Primer for fire-retardant paint shall be one that is recommended by the manufacturer of the paint.

B. Fungicide Agent shall be of a type that will not adversely affect the color or durability of the paint. The fungicide agent shall be added to the paint at the factory or mixed in the proportions recommended by the manufacturer. The amount of fungicide agent added shall be at least enough to render the fabric mildew-proof.

C. Block filler shall be a vinyl acrylic latex based filler coating that is acceptable for both interior and exterior use.

D. Thinner shall be a type that is recommended by the manufacturer of the paint, and shall be used in accordance with the manufacturer's instructions. Use odorless thinner with odorless enamel (TT-E-509). 
2.03 COLORS - Paint colors, if not indicated in the contract document, shall be as selected from paint manufacturer's color chips. Match existing colors where directed.

\section{PART 3 - EXECUTION}

\subsection{SURFACE PREPARATION}

A. General: Perform preparation and cleaning procedures in accordance with paint manufacturer's instructions and as herein specified, for each particular substrate condition.

Do not paint over dirt, rust, scale, grease, moisture, scuffed surfaces, or conditions otherwise detrimental to formation of a durable paint film.

B. Cementitious Materials: Prepare cementitious surfaces of concrete and concrete block, to be painted by removing efflorescence, chalk, dust, dirt, grease, oils, and by roughening as required to remove glaze.

C. Wood: Clean wood surfaces to be painted of dirt, oil, or other foreign substances with scrapers, mineral spirits, and sandpaper, as required. Sandpaper smooth those finished surfaces exposed to view, and dust off. After priming, fill holes and imperfections in finish surfaces with putty or plastic wood-filler. Sandpaper smooth when dried.

D. Ferrous Metals: Clean ferrous surfaces, which are not galvanized or shopcoated, of oil, grease, dirt, loose mill scale and other foreign substances by solvent or mechanical cleaning. abraded or corroded spots on shop-coated surfaces shall be wire brushed and touched up with the same paint as the shop coat.

E. Galvanized Surfaces: Clean free of oil and surface contaminants with nonpetroleum-based solvent.

F. Gypsum Board and Plaster Surface: Repair blemishes, irregularities, and damaged areas.

\subsection{APPLICATION}

A. General: Apply paint in accordance with manufacturer's directions. Use applicators and techniques best suited for substrate and type of materiel being applied. Spray method shall be used only when so specified by notes on drawings or special specifications for the project. 
B. Atmospheric Conditions: Apply water-base paint only when temperature of surfaces to be painted and surrounding air temperatures are between $50^{\circ} \mathrm{F}$ $\left(10^{\circ} \mathrm{C}\right)$ and $90^{\circ} \mathrm{F}\left(32^{\circ} \mathrm{C}\right)$, unless otherwise permitted by the paint manufacturer's printed instructions.

Apply solvent-thinned paint only when temperature of surfaces to be painted and surrounding air temperatures are between $45^{\circ} \mathrm{F}\left(7^{\circ} \mathrm{C}\right)$ and $95^{\circ} \mathrm{F}\left(35^{\circ} \mathrm{C}\right)$, unless otherwise permitted by the paint manufacture's printed instructions.

Do not apply paint in snow, rain, fog, or mist, or when relative humidity exceeds $85 \%$, or to damp or wet surfaces, unless otherwise permitted by paint manufacturer's printed instructions.

Painting may be continued during inclement weather if areas and surfaces to be painted are enclosed and heated within temperature limits specified by the paint manufacture during application and drying periods.

C. Rate of Application: The contractor shall assume the responsibility that the number of coats specified in the painting schedule will provide "full coverage" of underlying surfaces, and "full coverage" shall be acceptable. If, in the judgment of the Sandia Delegated Representative, "full coverage" is not accomplished in the number of coats specified, the contractor shall apply additional coats at no additional cost until "full coverage" accomplished. finish paints shall be applied without leaving drops sags, ridges, waves, voids, holidays, and defective brushing.

D. Color Coding of Sprinkler systems: Paint all exterior control valve indicator posts, water motor alarm and gongs, fire department connections, and sprinkler system trains through walls afire Protection Red.

Also, paint interior sprinkler system riser to ceiling line or to the first horizontal run, alarm valve, and all other related accessories "Fire Protection Red."

\subsection{CLEAN-UP AND PROTECTION}

Protect work of other trades, whether to be painted or not, against damage by painting and finishing work. Correct any damage by cleaning, repairing or replacing, and repainting, as acceptable to the Sandia Delegated Representative.

Upon completion of painting work, clean window glass and other paint-spattered surfaces. Remove spattered paint by proper methods of washing and scraping, using care not to scratch or otherwise damage finished surfaces.

\subsection{PAINTING SCHEDULE}


Surface $\quad \underline{\text { Primer }} \quad \underline{\text { First Coat }}$ Finish Coat

A. Wood (painted)

1. New
a.Interior
Fire Retardant
Fire Retardant Fire Retardant
b. Exterior TT-P-25
TT-E-489
TT-E-489

2. Previously Painted
a. Interior
Fire Retardant Fire Retardant
b. Exterior
TT-E-489
TT-E-489

B. Wood (varnished)

TT-V-86

TT-V-86

C. Ferrous Metal

1. New
a. Interior TT-P-636 or
TT-E-543
TT-E-509
b. Exterior
TT-P-86
TT-E-489 TT-E-489
TT-P-86

2. Previously Painted
a. Interior
TT-E-543
TT-E-509
b. Exterior
TT-E-489
TT-E-489

D. Galvanized Metal

1. New
a. Interior TT-P 641
TT-E-543
TT-E-509
b. Exterior TT-P-641
TT-E-489
TT-E-489

2. Previously Painted
a. Interior
TT-E-509
TT-E-509
b. Exterior
TT-E-489
TT-E-489

E. ' Concrete Block

1. New
a. Interior Block Filler
TT-E-543 or
TT-E-509 or
TT-P-1511
TT-P-1511
b. Exterior Block Filler
TT-P-19
TT-P-19

2. Previously Painted 

a. Interior
TT-E-543 or
TT-E-509 or
b. Exterior
TT-P-1511
TT-P-1511
TT-P-19
TT-P-19

F. Concrete and Stucco

1. New
a. Interior
TT-P-1511
TT-P-1511
b. Exterior
TT-P-19
TT-P-19

2. Previously Painted
a. Interior
TT-P-1511
TT-P-1511
b. Exterior
TT-P-19
TT-P-19

G. Gypsum Wallboard and Plaster

1. New

TT-P-650

TT-P-1511

TT-P-1511

2. Previously Painted

TT-P-1511 TT-P-1511

H. Canvas-Covered insulation on pipe

TT-P-29

TT-P-29 and ductwork

w/ fungicidal

agent

End Of Section 09900 


$$
\begin{aligned}
& \text { 구 } \\
& \text { 矛 } \\
& =\Omega \\
& \text { 모 } \\
& \text { ‥ } \\
& \text { 음 }
\end{aligned}
$$




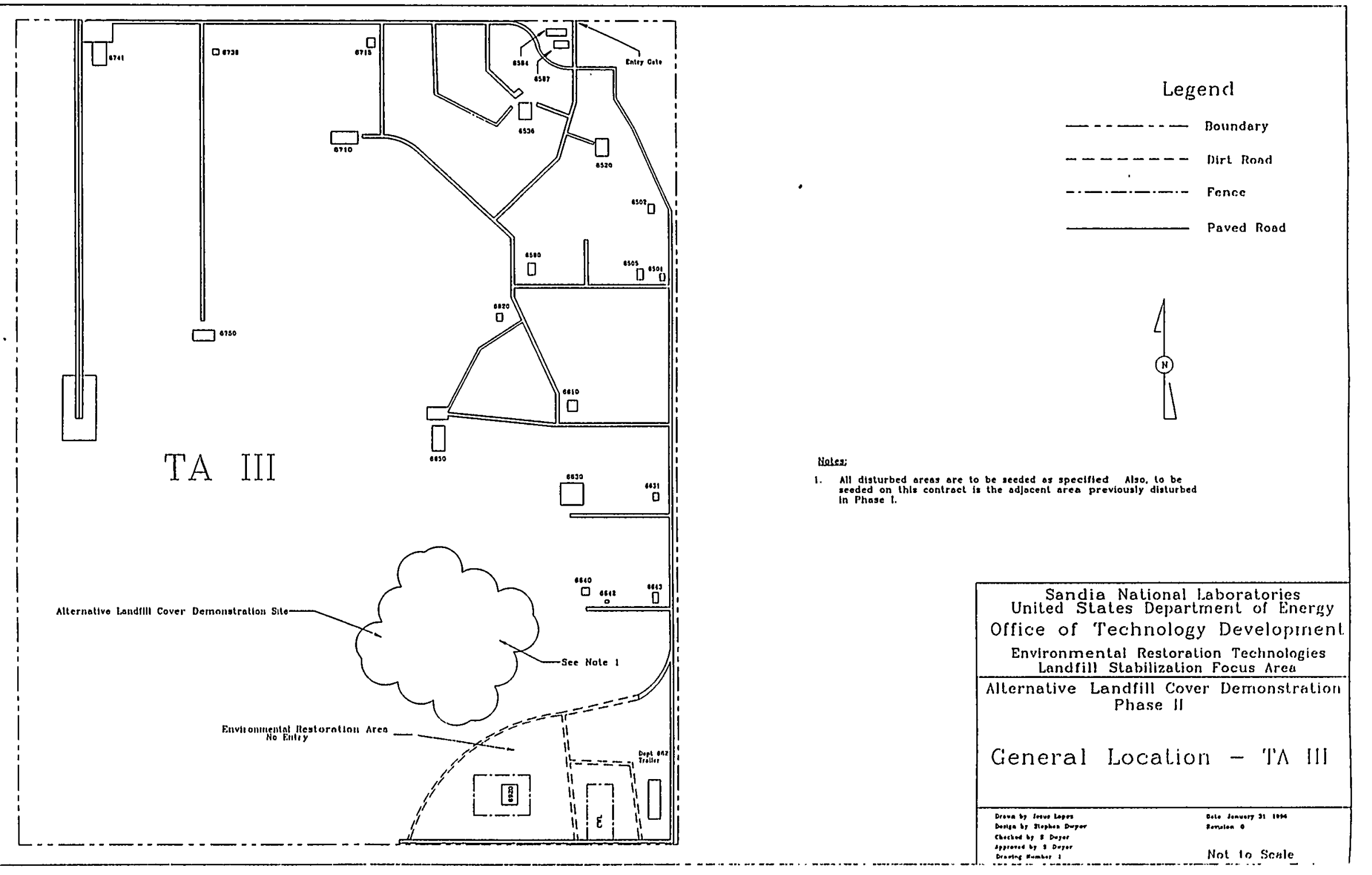




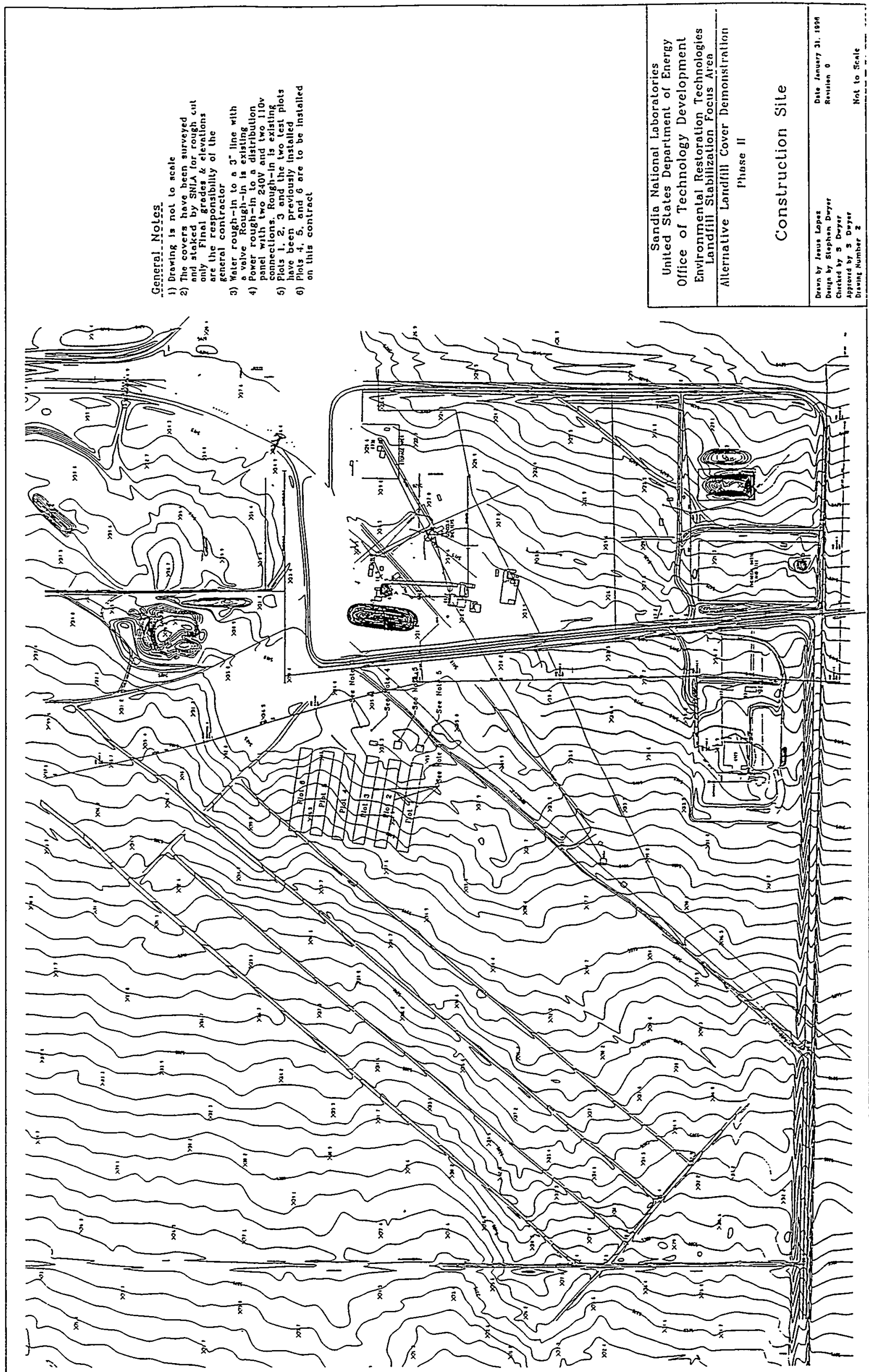




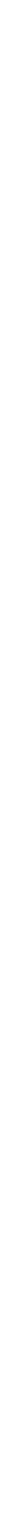


5\% GRADE:

See Drawing 5

lor Enlarged Seclion

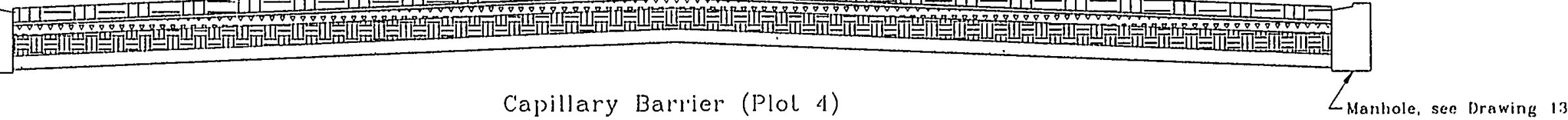

Capillary Barrier (Plol 4) Seclion

Manhole, see brawing $1:$

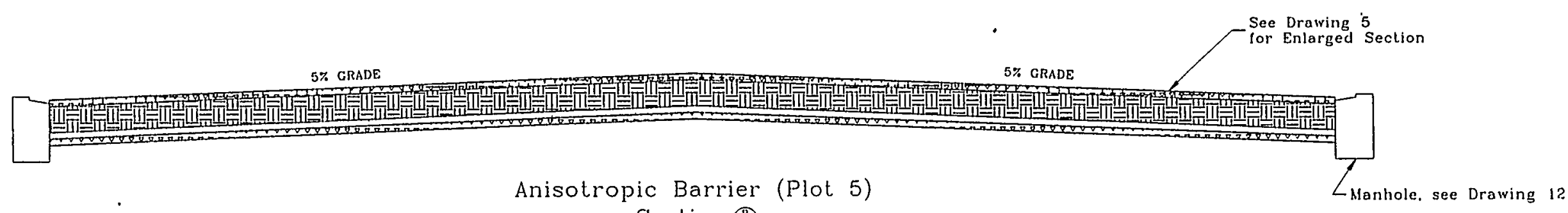

Section (1:

5\% GRADE

5\% GRADE

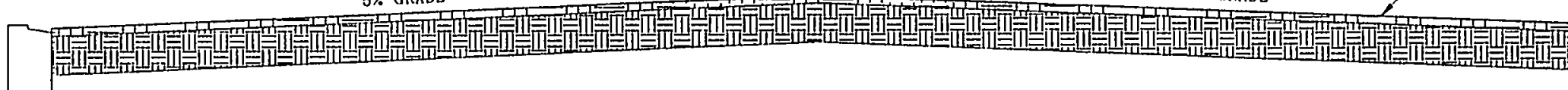

See Drawing 5

arged Section

Evapotranspiralion Soil Cover (Plol 6)

Seclion (갈

Sandia National Laboratories

Uniled States Department of Energy Office of Technology Development

Environmental Restoration Technologie

Landfill Stabilization Focus Area

Alternative Landfill Cover Demonstration Phase II

Cover Closs Sections

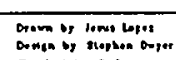

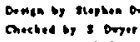
Not in Scale ..... 


\section{Anisolropic Barrier (Plol 5)}

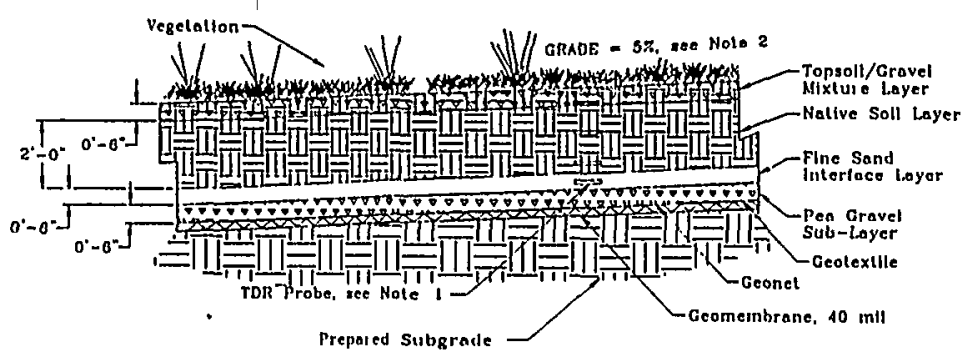

Evapotranspiration Soil Cover (Plot 6)

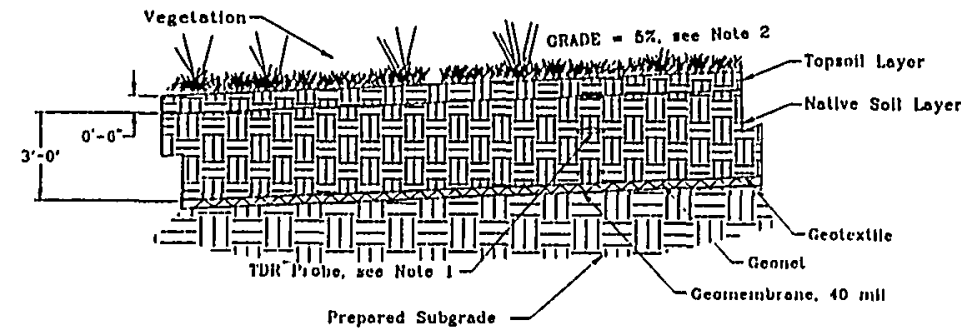

\section{Legend}

- TDR Probe

\section{Capillary Barrier (Pløt 4)}

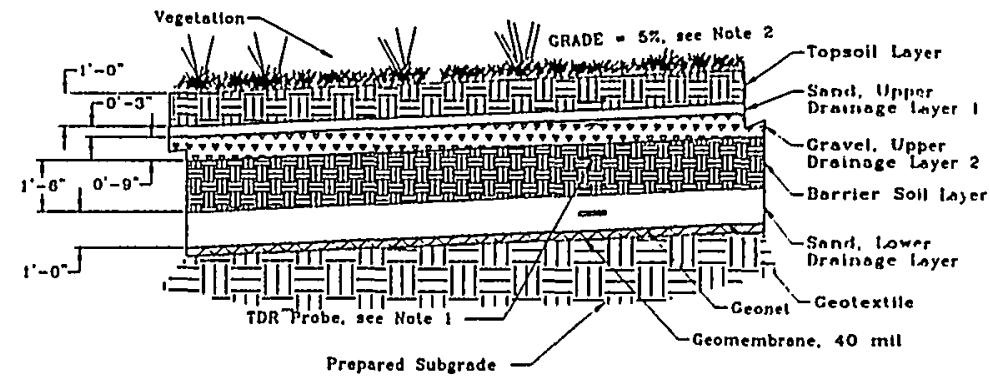

Noles:

Shows approximale TDR depth locations for each

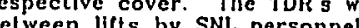
allow time for this installation. Probes sliown for

2) All layers within each cover will slope $5 \%$

United Sandia National Laboratories Office of Technology Developmen Environmental Restoration Technologies Alternative Landfill Cover Demonstration Phase II

Typical Cover Cross Sections and

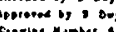

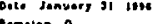

Not in Sonle 


\section{Noles:}

1) Arrangement typical to each end of the cover plots.

pipes to have

miniuum slope of IX" per loot to drain.

3. Locate drains from concrete curbs to lee out just

the south of each respective manhole as

loward the existing waler collection gallery.

zach drainage pipe is to rim independenty

are to be field lestod by controctor aller

installation to ensure drainage capability.

4. Core drill the concrete wall in the water

collection gallery to allow for penetration

of eacl drainage pipe. The drainage plpes
shall protrude into the gallery a nin. of 1 .
Seal around each penctrotion - walertighi.

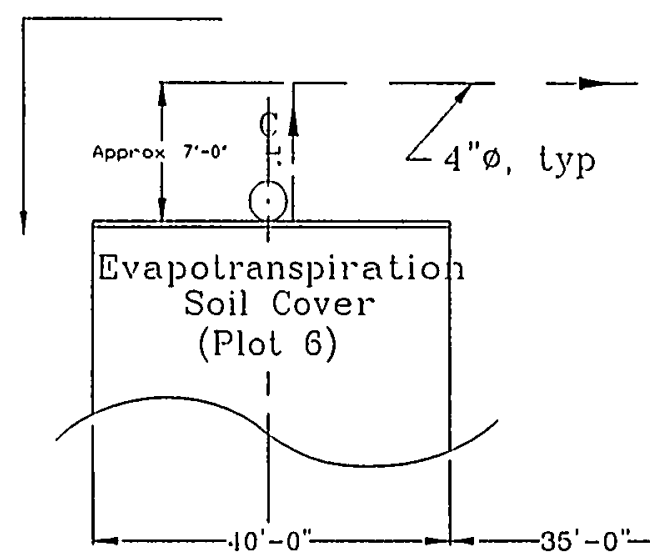

$-35^{\prime}-0^{\prime \prime}$
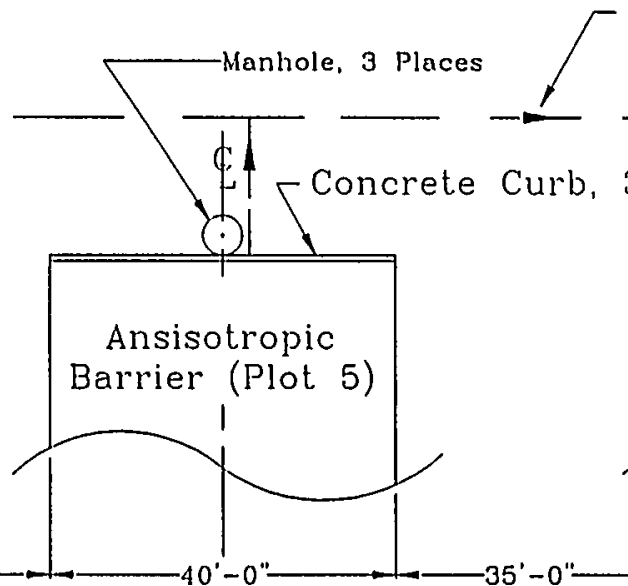

Direction

of Flow
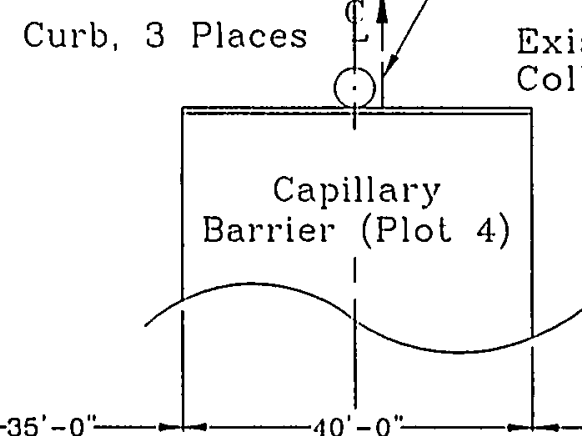

Pan View

Concrele Curb Drain Delail $-35^{\prime}-0$

\section{Legend}

- - Center line of Collection Pipes

$\longrightarrow$ (3)
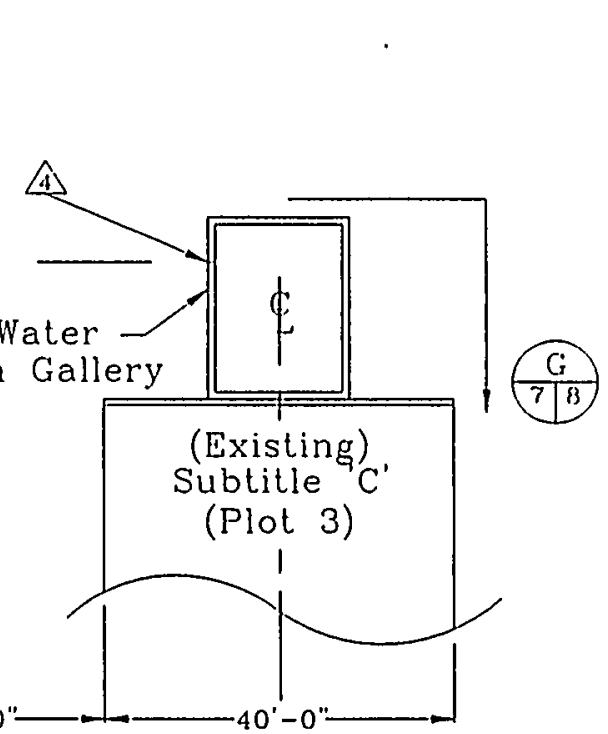

Sandia National Laboratories
United States Department of Energy Office of Technology Developinent.

Environmental Restoration Technologies

Alternative Landfill Cover Dernonstrátion Phase II

Plan View of Waler Collection Pipe Systern 


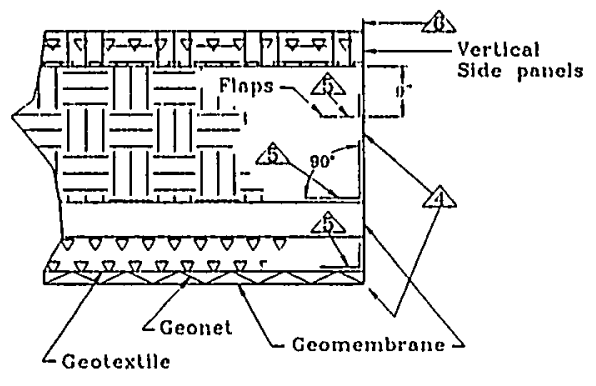

Anisolropic Barrier (Plot 5)

Verlical Side Delail $\frac{2}{010}$

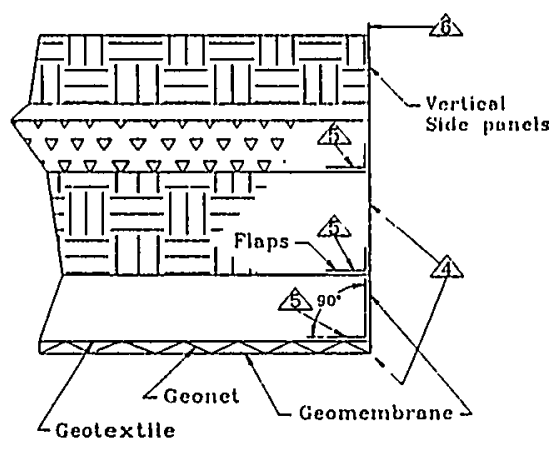

Capillary Barrier (Plot 4)

Vertical Side Detail $\frac{1}{6019}$

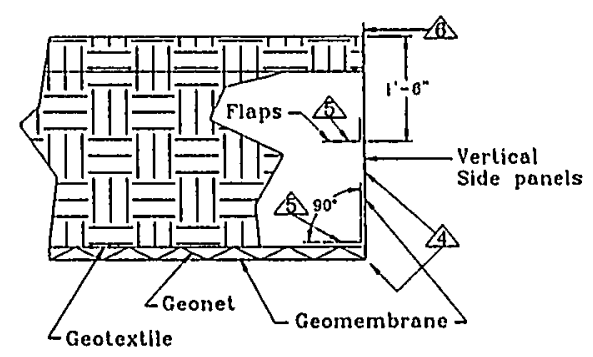

Evapolranspiralion Cover (Pol 6)

Verlical Side Delail $\frac{3}{610}$

Noles:

1) Vertical side detail is continuous olong full length of 2) Vertical side detail is typical to each side of each test 3) The vertical side panels ore to be welded to the vertical end panels (4 corners per test cover plot) to make

(1) The geomembrane (no welded seam Q bend) is continuous the vertical side panels.

Ait Finp made of the same material as the verticnl sille pricele continuous the flaps are to extend out into the cover $I^{\prime}-n$ The welds must be above the portion extendng a tolerance of $+5^{\circ},-0^{\circ}$. Any upward slope of the flaps is unacceptable. These haps run continuously along the full length of the vertical side panels. The locations oi haps are unless dimensioned otherwise.

The vertical side panels are to extend to daylight

Sandia National Laboratories
United States Department of Energy
Office of Technology Development.
Environmental Restoration Technologies
Landfill Stabilization Focus Area



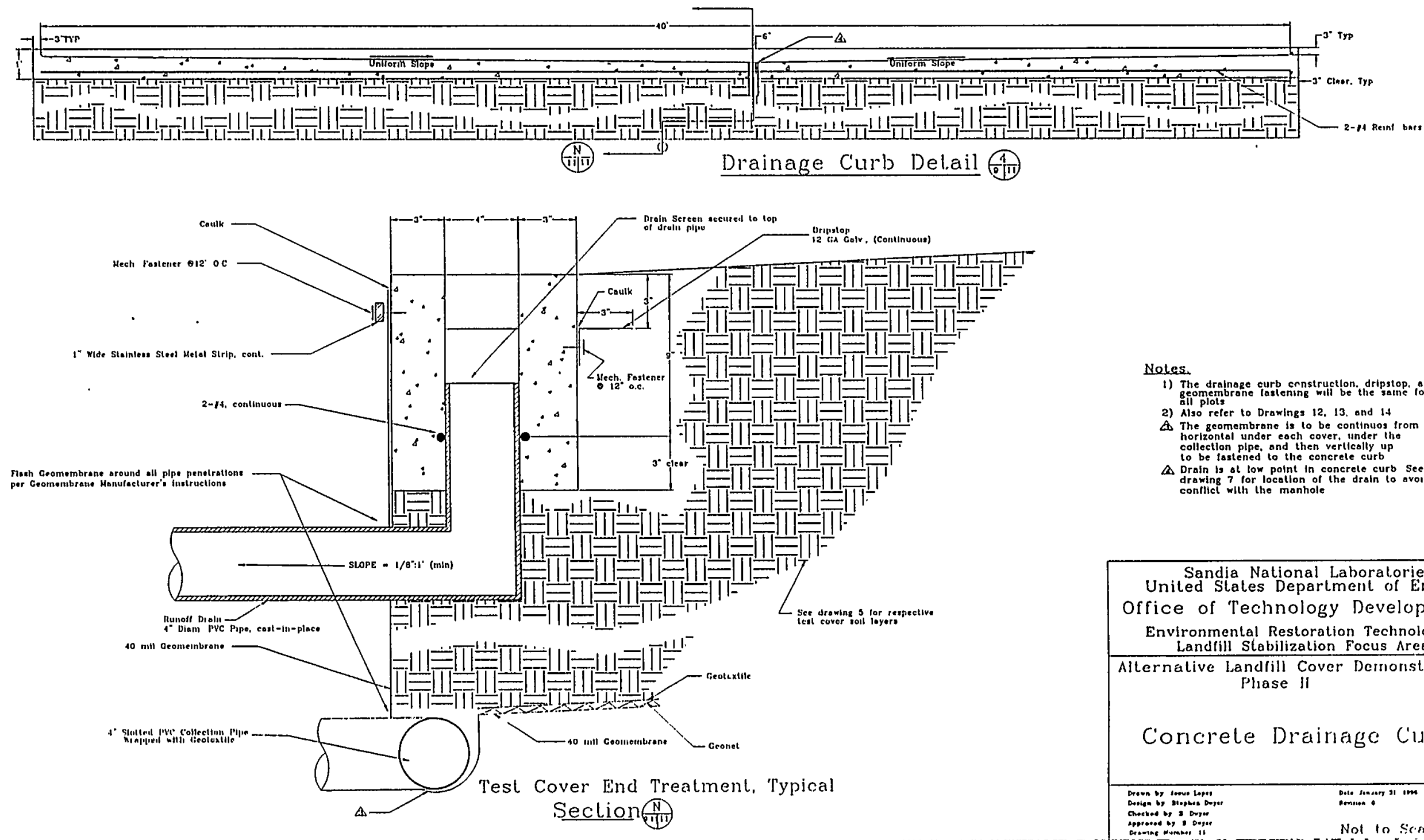

Netes.

i) The drangoge curb ennstructlon. dripstop. and
geomentrone lastening will be the satne lor

2) Also reter 10 Denuing: 12 , 13 and 14

a) The geomembrane is 10 be and 14 irom horlzontal under each cover, under tho to be lastened to the concrele curb

$\Delta$ Drain is ot low point in concrele curb See
drawing 7 lor locatlon of the dratn to avoid confliel with the manhole

Sandia National Laboralories United States Department of Energy Office of Technology Developrnen Environmental Restoration Technologies Landfill Stabilization Focus Area Alternative Landfill Cover Demonstration Phase II

Concrele Drainage Curb 


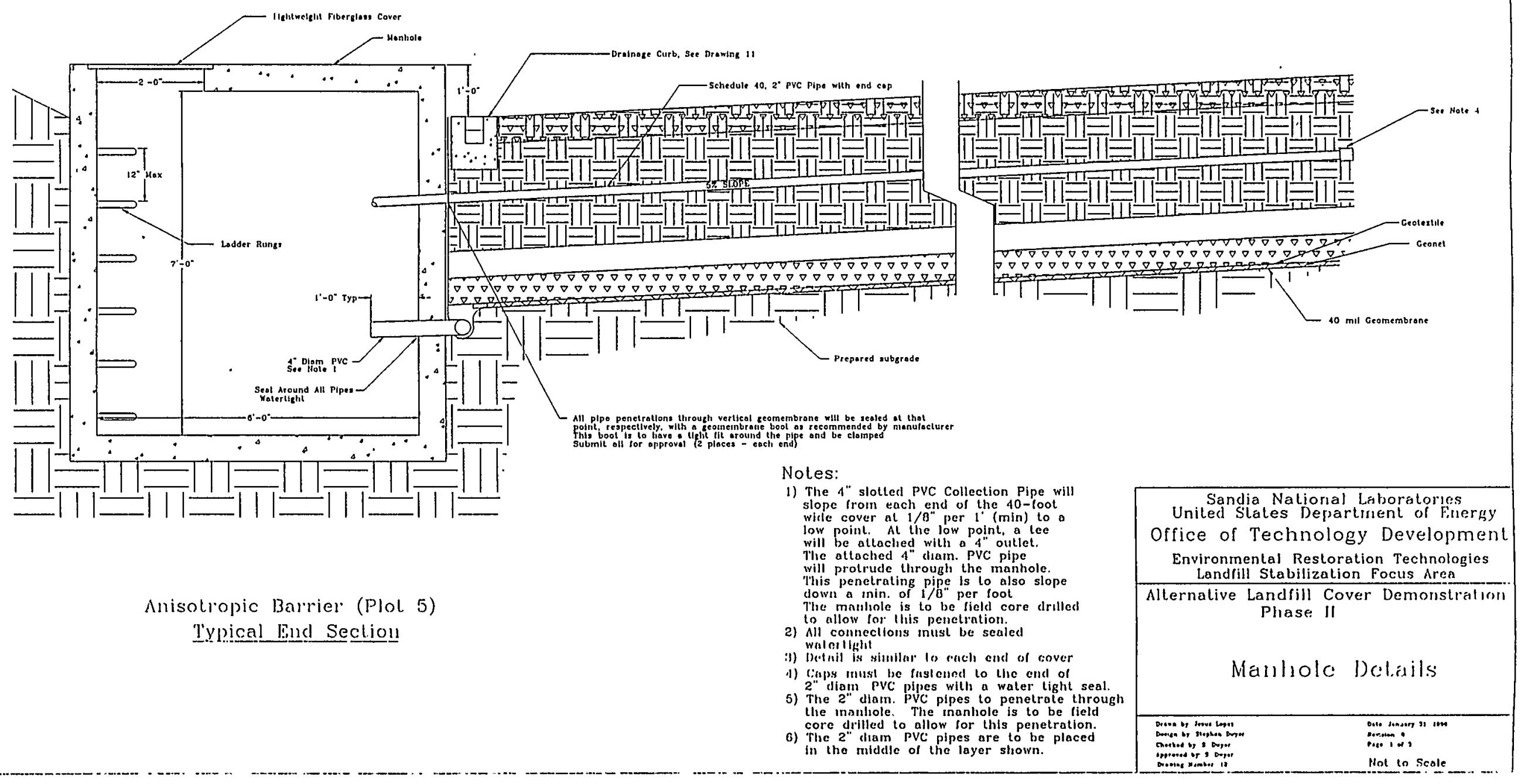



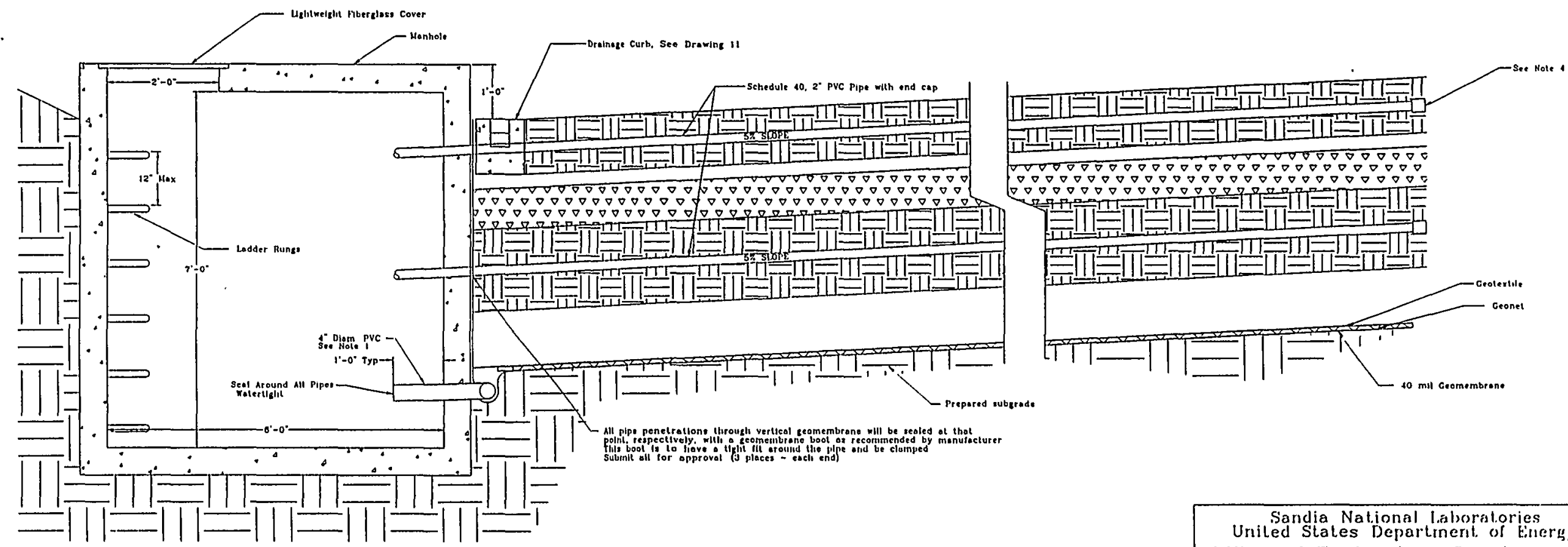

Capillary Barrior (Plol 4)

I'ypican find Sogtion?

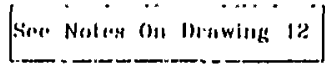

Sandia National Laboratories
Uniled States Departinent, of tiler Environmental Restoration Technologies Landfill Stabilization Focus Area Alternative Landfill Cover I)emonstration Phase II

Manhole Delails

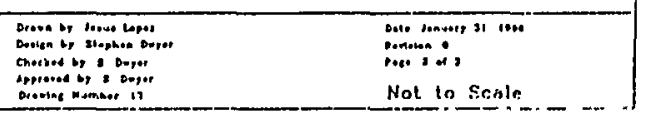




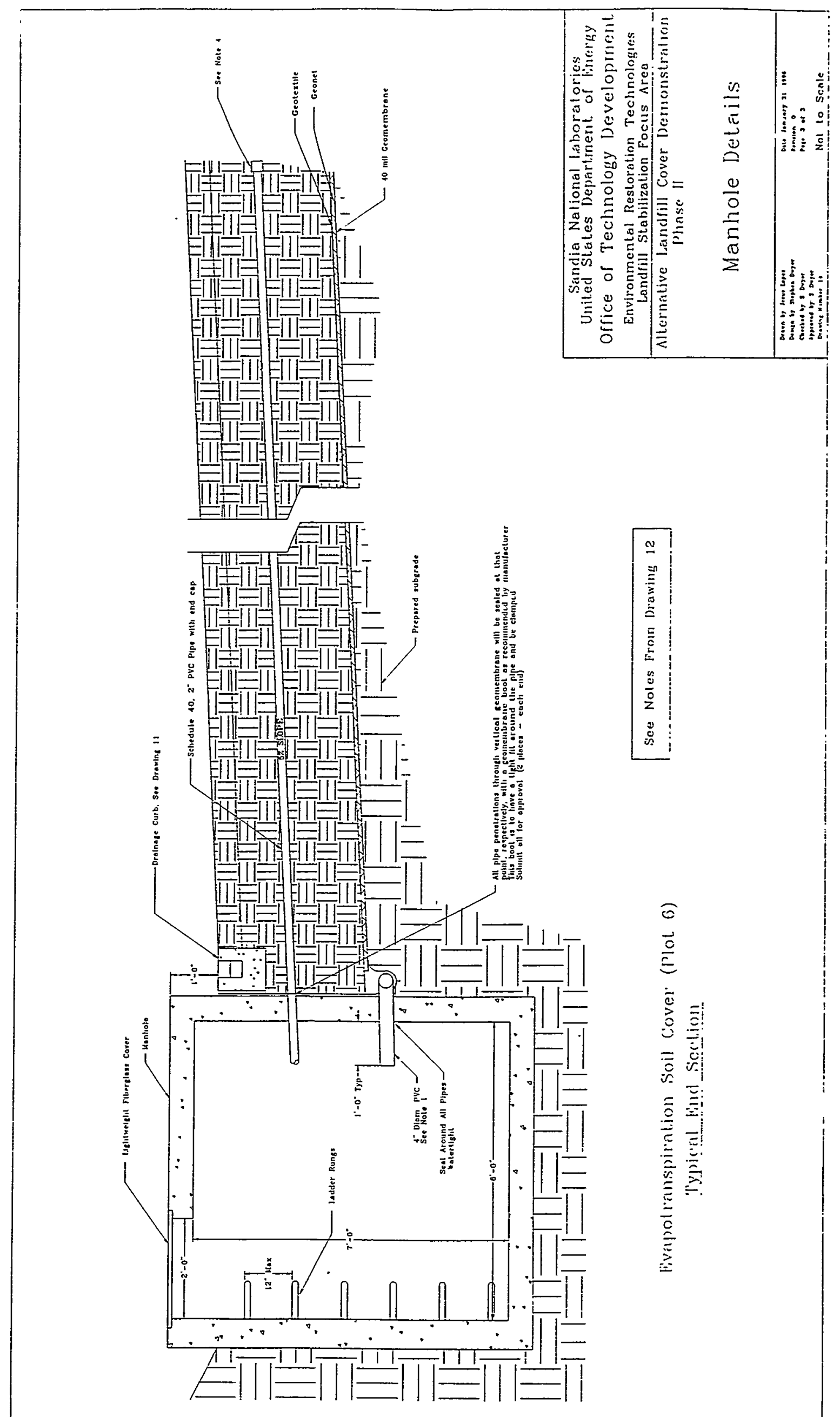


ATTACHMENT E

Phase II - Specifications 
Sandia Nationa7 Laboratories

\section{Alternative Landfill Cover Demonstration \\ Phase II \\ Specifications}

$1-31-96$

prepared by:

Stephen F. Dwyer 


\section{SPECIFICATIONS}

\begin{tabular}{|c|c|}
\hline DIVISION 1 & GENERAL REQUIREMENTS \\
\hline SECTION 01000 & OVERVIEW OF TEST CONFIGURATION \\
\hline SECTION 01300 & DESCRIPTIVE SUBMITTALS \\
\hline DIVISION 2 & SITE WORK \\
\hline SECTION 02110 & CLEARING AND GRUBBING \\
\hline SECTION 02200 & EARTHWORK \\
\hline SECTION 02210 & RIPRAP \\
\hline SECTION 02725 & MANHOLES \\
\hline SECTION 02776 & GEOMEMBRANE \\
\hline SECTION 02778 & GEOTEXTILES \\
\hline SECTION 02779 & DRAINAGE NETS \\
\hline SECTION 02780 & TEXNET \\
\hline SECTION 02900 & SEEDING \\
\hline SECTION 02901 & SPRINKLER SYSTEM \\
\hline DIVISION 3 & CONCRETE \\
\hline SECTION 03300 & CAST-IN-PLACE CONCRETE \\
\hline DIVISION 5 & METALS \\
\hline SECTION 05120 & STRUCTURAL STEEL \\
\hline SECTION 05500 & METAL FABRICATIONS \\
\hline DIVISION 9 & FINISHES \\
\hline SECTION 09900 & PAINTING \\
\hline
\end{tabular}

GENERAL REQUIREMENTS

OVERVIEW OF TEST CONFIGURATION

DESCRIPTIVE SUBMITTALS

SITE WORK

CLEARING AND GRUBBING

EARTHWORK

RIPRAP

MANHOLES

GEOMEMBRANE

GEOTEXTILES

DRAINAGE NETS

TEXNET

SEEDING

SPRINKLER SYSTEM

CONCRETE

CAST-IN-PLACE CONCRETE

METALS

STRUCTURAL STEEL

METAL FABRICATIONS

FINISHES

PAINTING 


\section{SECTION 01000}

\section{OVERVIEW OF TEST CONFIGURATION}

Phase II of the Alternative Landfill Cover Demonstration (ALCD) will continue the sideby-side installation of landfill cover test plots begun in Phase I. Plots 1, 2, and 3 were constructed in Phase I. Plots 4, 5, and 6 will be constructed in Phase II. The plan dimensions of each cover will be 40 -feet wide by 300 -feet long. The 300 -foot length will be divided into two halves - one sloping at 5\% toward the east with the other half sloping at $5 \%$ toward the west. All layers within each landfill cover test plot will also slope at $5 \%$. The north-south slope on each cover (and all layers within each cover) will be $0 \%$. The eastern half of each cover will have a sprinkler system installed in them to simulate various precipitation events during stress testing.

The covers were designed and will be constructed such that the cut volume of soil will approximately equal the fill required to construct the covers. Therefore, no borrow fill will be required. Refer to the contract drawings.

Plot 4 will be a Capillary Barrier. This cover system consists of 4 primary layers: (1) a surface or topsoil layer; (2) an upper drainage layer; (3) a barrier soil layer; and (4) a lower drainage layer. See figure 1. All layers will be constructed at a slope angle of $5 \%$ to enhance surface runoff and lateral drainage. The topsoil layer will have a minimum thickness of 12 inches. This surface layer is placed to enhance evapotranspiration (ET), protect against desiccation of the barrier soil layer, and provide a medium for growth of vegetation. This vegetation increases ET and protects against surface erosion. The upper lateral drainage layer will consist of a minimum thickness of 9 inches of gravel overlain by 3 inches of sand. The sand serves as a graded filter to prevent topsoil from clogging the drainage layer. The gravel allows for lateral drainage of any water that has percolated through the topsoil. The barrier soil layer and lower drainage layer comprise the capillary barrier. The barrier soil layer will be compacted soil with a minimum thickness of 18 inches. The lower drainage layer will be a minimum thickness of 12 inches of sand.

Plot 5 will be another capillary barrier referred to as the Anisotropic Barrier. This cover system consists of 4 layers: (1) a top vegetation layer; (2) a cover soil layer; (3) an interface layer; and (4) a sublayer. See figure 1. The vegetation layer will have a minimum thickness of 6 inches. It will be comprised of a mixture of local topsoil and pea-gravel. The gravel to soil mixture by weight $(0.25=$ dry weight of gravel $/$ dry weight of soil). This layer will encourage evapotranspiration, allow for vegetation growth, and reduce surface erosion. The cover soil layer will have a minimum thickness of 24 inches. It will be comprised of native soil. Its function is to allow for water storage and eventual evapotranspiration, as well as, serve as a rooting medium. The interface layer will have a minimum thickness of 6 inches of fine sand. This layer serves as a filter between the overlying soil and the underlying gravel. It also serves as a drainage layer to laterally divert water that has percolated through the cover soil. The sublayer will have a 
minimum thickness of 6 inches of pea-gravel. It serves as a capillary break. The interface layer and sublayer combined also serve a dual purpose as bio-barriers.

Plot 6 will be a monolithic soil cover referred to as an Evapotranspiration Cover. See figure 1 . The cover will consist of only one layer - soil. It will have a minimum thickness of 36 inches. The bottom 30 inches will be compacted while the top 6 inches of topsoil will be loosely placed. The soil will allow for water storage combined with the vegetation will increase evapotranspiration. Refer to the contract drawings for details of the above described test plots.

The plots will be instrumented to determine all variables in the water balance \{precipitation (P), surface runoff (R), lateral drainage (D), soil water storage (S), and percolation or infiltration (I)\} equation with the exception of evapotranspiration (E) which will be estimated by solving the mass balance equation.

$$
\mathrm{E}=\mathrm{P}-\mathrm{I}-\mathrm{R}-\mathrm{D}-\Delta \mathrm{S} \quad \text { [Equation 1] }
$$

Precipitation and any water added to the covers with a sprinkler system will be quantified using a met station and/or flow meter on the sprinkler system. All water passing through the covers will be laterally routed to respective collection underdrain systems. Surface water runoff will be collected at the end of each respective slope with a gutter system. This collected water will then be routed through measurement devices to quantify it. TDR probes will be strategically located throughout the covers at various depths to determine soil moisture contents that can in turn be interpolated to determine water storage capacities. Thermocouples will also be installed at various depths and plan locations to determine soil temperature. Refer to section 8.0 of this Test Plan for descriptions of the instrumentation to be installed. The drawings in Attachment A show locations and details of the instrumentation. This instrumentation will be installed by SNL personnel. Sheds will be located on-site to house the data acquisition equipment to protect it from the weather. A met station will be located on-site to record all pertinent meteorological data.

The site has been designed so as to minimize runoff and run-on. Civil engineering cut/fill techniques were used to design the site. The cut taken from the site approximately equals the fill soil volume required to build the test plots. This ensures that soil specific to the sight is used for layers of the covers. Sand and gravel will require being purchased offsite and trucked on-site. Drainage is provided for around the perimeter of each cover.

A sprinkler system will be installed in the east half of each test plot. This system will be installed as designed in the contract drawings using the materials specified. Utilities at the site including power and water are roughed-in. The water is potable. After all earthwork activities are complete including trenching - the test plots, as well as, all disturbed earthen areas will be seeded with native vegetation as specified unless noted otherwise. The seed will also be applied and fertilized as specified. 


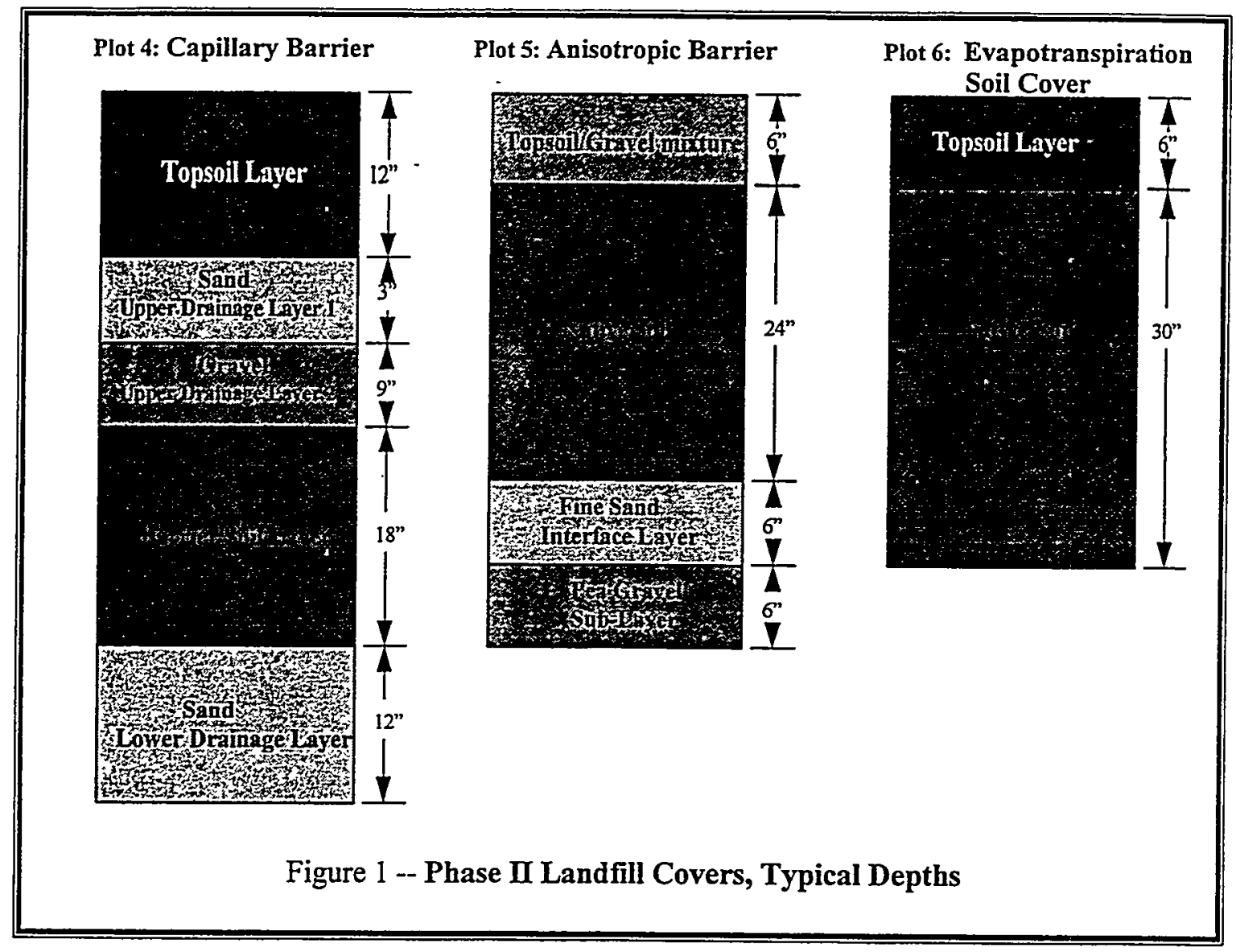

End of Section 01000 


\section{STANDARD SPECIFICATION \\ SECTION 01300 \\ DESCRIPTIVE SUBMITTALS}

\section{PART 1 - GENERAL}

\subsection{DESCRIPTION OF WORK}

Section Includes: This section includes administrative and procedural requirements for submittals required for performance of the work, including:
A. Tvpe A - Shop Drawings
B. Type B - Catalog Data
C. Type $\mathrm{C}$ - Installation Instructions
D. Type D - Operating Manual, Maintenance Instructions and Parts List
E. Type E - Internal Wiring Diagrams
F. Type S - Special

\subsection{PURPOSE OF SUBMITTAL REVIEW}

A. General Contractor Responsibility: The General Contractor's responsibility with respect to submittals shall be to review, stamp, and submit all submittals in the proper format and in a timely manner. The General Contractor shall be responsible for the following items and shall not submit a request for approval of said items but shall be ultimately and solely responsible for them:

1. Field verification and confirmation of all dimensions for proper fit of all materials. Sub-contractors' requests for field verification of dimensions shall be addressed by the General Contractor prior to submitting shop drawings to Sandia National Laboratories (the Sandia Delegated Representative).

2. Field verification and confirmation of quantities of materials.

3. Fabrication processes and techniques of construction.

B. Sandia Delegated Representative Responsibilitv: Submittals shall be reviewed by the Sandia Delegated Representative for the sole purpose of checking general conformance with the design concepts of the project and general compliance with the contract documents. 
Any action shown is subject to the requirements of the contract documents including plans and specifications and does not relieve the General Contractor from compliance with all provisions and requirements therein. Approval of a submittal does not relieve the General Contractor of his responsibility for correcting any errors which may exist in the submittal or for meeting the requirements of the contract documents.

\section{PART 2 - MATERIALS}

\subsection{DESCRIPTIVE SUBMITTAL LISTS}

A. Submittal Codes: The following abbreviations: Arch., Civ., Struc., Mech., Elec., or Con. - identify the respective Architectural, Civil, Structural, Mechanical, Electrical or Controls discipline for which information is being requested.

B. Reference Numbers: Reference numbers are to be assigned to each submittal.

C. Submittal Types: The descriptive submittal types are used to identify the type of information being requested. They are defined below and are marked on the "Descriptive Submittals" review form.

1. Type A - Shop Drawings: Detailed drawings prepared specifically for the project and used for fabrication and/or assembly of material or equipment.

a. the Sandia Delegated Representative design/construction drawings shall not be reproduced and used for shop drawings.

b. Shop drawings shall be prepared accurately and to scale. Encircle deviations from the contract documents.

c. Shop drawings shall include dimensions, identification of products and materials, notation of special coordination requirements, notation of dimensions established by field measurements and shall indicate compliance with specified standards.

d. Shop drawings shall be submitted on sheets at least 8-1/2" $\times 11$ " (216 $\mathrm{mm} \times 279 \mathrm{~mm}$ ) but no larger than 24" x 36" (610 mm x $914 \mathrm{~mm})$.

2. Type B - Catalog Data: Manufacturer's published literature describing the salient features of the product (e.g. size, weight, performance, code conformance, capacity, physical size, pictures, ratings, and construction features). Clearly highlight specified items and optional features such that there is no misunderstanding as to what precisely is being proposed.

3. Type C - Installation Instructions: Manufacturer's information explaining the preparation and installation of material and equipment, including: 
a. Outline drawings showing major components and dimensional service connection points.

b. Service connection requirements such as electrical fuse or circuit breaker size and type, pipe size and type, air duct, critical ambient conditions (cooling, heating), foundation requirements, and special precautions.

c. Adjustment requirements that must precede operation, including initial alignment procedures, leveling, purging, charging, and lubrication.

4. Type D - Operating Manual, Maintenance Instructions, and Parts List: Complete calibration procedures and operating instructions including all necessary pre-start up checks and safety precautions.

a. Provide instructions for step-by-step preventive maintenance and minor field repairs that will ensure optimum dependable performance, longevity, and life cycle cost.

b. Provide manufacturer's compiled parts list for maintenance and repair.

c. Provide a symptom, diagnostic, and corrective action schedule.

5. Type E - Internal Wiring Diagrams: Internal wiring and elementary (ladder) diagram for factory pre-wired equipment.

a. Include control diagrams that show the relative position of each component as a system diagram.

b. Include any additional information requested in the Mechanical and Electrical specifications for a particular product.

6. Type S-Special: Special information, samples and others as indicated.

a. Include actual color, texture, and pattern when submitting samples.

b. Indicate product sources and availability as well as manufacturer's name.

D. Asbestos Free and Lead Paint Free Certification Submittal: It is required that all products used on the construction project be "free of asbestos" and "free of lead paint." In addition to the above submittal types, for every product on which a submittal is requested, the Contractor shall also submit written certification from the product manufacturer stating that the products to be used on this project are "free of asbestos" and "free of lead paint."

\section{PART 3 - EXECUTION}


A. Coordination: Coordinate preparation and processing of submittals with performance of construction activities. Transmit each submittal in advance of performance of related construction activities.

1. Coordinate each submittal with fabrications, purchasing, testing, delivery, other submittals and related activities that require sequential activity.

2. Coordinate transmittal of different types of submittals for related materials so processing will not be delayed by the need to review related submittals concurrently.

The Sandia Delegated Representative reserves the right to withhold action on a submittal requiring review of related submittals until the related submittal information is received.

B. Review Time: The Sandia Delegated Representative may take two weeks for initial review. The Sandia Delegated Representative may also take additional time if processing must be delayed to permit review of related subsequent submittals. Allow additional time for processing of resubmittals.

C. Submittal Packaging: Bindable submittals, for each discipline, shall be three hole punched and bound into a card stock binder with flexible metal clips:

1. The submittal binder shall be stamped on the lower right hand corner of the cover with an approximately 4" x 4" (102 mm x $102 \mathrm{~mm})$ identification stamp as described below:

"Discipline: " (e.g., Arch., etc.)

"Contract Number: " (e.g., TW-0030)

"Descriptive Submittal Reference Number: through 15 and 19 through 22)

"General Contractor Information:"

"Company Name:

"Company Phone Number:

"General Contractor Reviewer Signature:

"General Contractor Reviewer Printed Name:

2. The General Contractor shall submit descriptive submittals to the Sandia Delegated Representative only after General Contractor review. The General Contractor's Reviewer shall sign his name on the identification stamp on the cover of the binder indicating General Contractor's approval of the submittal and acknowledgment as to the appropriateness of the material or information submitted for use in construction. 
Unstamped, unsigned, or otherwise incomplete submittals shall not be accepted by the Sandia Delegated Representative.

3. Submittals shall be grouped together with related products of the same discipline when possible (e.g., Finishes - vinyl floor tile, floor tile adhesive, vinyl base, ceiling tile, ceiling tile grid, carpet tile, carpet tile adhesive, ceramic tile, ceramic tile adhesive). The requested submittal types for a particular item should always be complete and submitted together. Do not submit one type of submittal apart from other types of submittals for the same product. Each submittal shall be legible and grouped together in the following order for each product requested:

a. Asbestos and Lead Paint Free Certification(s)

b. Submittal Type A - Shop Drawings. Fold shop drawings down to 8$1 / 2^{\prime \prime} \times 11^{\prime \prime}(216 \mathrm{~mm} \times 279 \mathrm{~mm})$ and insert into submittal package.

c. Submittal Type B - Catalog Data

d. Submittal Type C - Installation Instructions

e. Submittal Type D - Operating Manual, Maintenance Instructions and Parts List

f. Submittal Type E - Internal Wiring Diagrams

g. Submittal Type S - Special: (Only bindable special submittals including color charts and fabric swatch charts.) Unbindable special submittals shall be submitted under separate cover and per product specification.

D. SUBMITTAL DELIVERY: For all types of submittals except samples, transmit the number of submittals needed for the General Contractor's purposes plus two (2) copies to be retained by the Sandia Delegated Representative. Submittals received from sources other than the General Contractor shall not be accepted. Incomplete, improperly packaged and/or submittals which have not been stamped by the General Contractor shall be returned without action. When submitting samples, submit two (2) sets of samples for review unless otherwise requested.

1. Deliver descriptive submittals to the Sandia Delegated Representative within 15 calendar days after the Notice to Proceed, except: 1) as may be stipulated elsewhere in this Contract, or 2) for "original drawing(s)" prepared by the General Contractor for specialty systems, such as: HVAC Controls, fire protection sprinklers and/or lightning protection system, which shall be delivered within 30 calendar days after the Notice to Proceed.

2. Material and/or equipment shall not be installed until requested submittals pertaining to said material and/or equipment have been approved. 
E. SUBMITTAL REVIEW: Submittals will be reviewed and stamped with a self-explanatory action stamp. The stamp will be appropriately marked, as follows, to indicate the action taken:

1. Final Unrestricted Release: When submittals are marked "Approved", that part of the work covered by the submittal may proceed provided it complies with requirements of the contract documents; final acceptance will depend upon that compliance. The term "Approved" shall only indicate that there are no exceptions taken to the submittal.

2. Final But Restricted Release: When submittals are marked "Approved Except as Noted," that part of the work covered by the submittal may proceed provided it complies with notations or corrections on the submittal and requirements of the contract documents; final acceptance will depend upon that compliance.

3. Returned for Resubmittal: When submittal is marked "Not Approved, Revise and Resubmit", do not proceed with that part of the work covered by the or prepare a new submittal in accordance with the notations and resubmit without delay. Repeat as necessary to obtain a different action mark.

\section{PART 4 - ITEMS TO BE SUBMTTED}

\subsection{ITEMS TO BE SUBMTTED}

The following items are to be submitted as a minimum:

\begin{tabular}{|l|c|c|c|c|c|l|}
\hline \multicolumn{1}{|r|}{ Type } & A & B & C & D & E & \multicolumn{1}{|c|}{ S } \\
\hline Item & & & & & & \\
\hline Sprinkler System & $\bullet$ & $\bullet$ & & $\bullet$ & & \\
\hline Seed & & $\bullet$ & $\bullet$ & & & \\
\hline Fertilizer & & $\bullet$ & $\bullet$ & & & \\
\hline Mulch & & $\bullet$ & $\bullet$ & & & $\begin{array}{l}\text { Including } \\
\text { tackifier }\end{array}$ \\
\hline Reinforced. Concrete & $\bullet$ & $\bullet$ & & & & $\begin{array}{l}\text { Conc. \& Reinf. } \\
\text { Steel }\end{array}$ \\
\hline Geomembrane & $\bullet$ & $\bullet$ & $\bullet$ & & & $\begin{array}{l}\text { Installation } \\
\text { Layout }\end{array}$ \\
\hline Drainage Net & $\bullet$ & $\bullet$ & $\bullet$ & & & $\begin{array}{l}\text { Installation } \\
\text { Layout }\end{array}$ \\
\hline Geotextile & $\bullet$ & $\bullet$ & $\bullet$ & & & $\begin{array}{l}\text { Installation } \\
\text { Layout }\end{array}$ \\
\hline Gravel & & $\bullet$ & & & & $\begin{array}{l}\text { Particle Size } \\
\text { Distribution, } \\
\text { Hydraulic } \\
\text { Conductivity }\end{array}$ \\
\hline
\end{tabular}




\begin{tabular}{|c|c|c|c|c|}
\hline & & & & Test Results \\
\hline Sand & & - & & $\begin{array}{l}\text { Particle Size } \\
\text { Distribution, } \\
\text { Hydraulic } \\
\text { Conductivity } \\
\text { Test Results }\end{array}$ \\
\hline $\begin{array}{l}\text { Soil Compaction } \\
\text { Techniques }\end{array}$ & & - & & \\
\hline $\begin{array}{l}\text { Soil/Gravel Mixing } \\
\text { Method }\end{array}$ & & - & & \\
\hline Riprap & & $\bullet$ & & \\
\hline Slotted PVC Pipe & & $\bullet$ & & \\
\hline PVC Pipe & & $\bullet$ & & \\
\hline Adhesives & & $\bullet$ & & \\
\hline Paint & & $\cdot$ & $\cdot$ & \\
\hline Manholes & 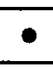 & $\bullet$ & $\bullet$ & Waterproofing \\
\hline Equipment & & $\bullet$ & & $\begin{array}{l}\text { All equip. to be } \\
\text { used, where, \& } \\
\text { how. }\end{array}$ \\
\hline Epoxy Coating & & $\bullet$ & $\bullet$ & \\
\hline Anchors & - & $\bullet$ & & \\
\hline Ashphaltic Coating & & $\cdot$ & - & \\
\hline
\end{tabular}

END OF SECTION 01300 


\section{SECTION 02110}

\section{CLEARING AND GRUBBING}

\section{DESCRIPTION}

1 This work consists of clearing, grubbing, removing, and disposing of vegetation and debris within the limits of the right of way, easement areas borrow pits, and other areas shown in the Contract or required by the work. Vegetation and objects designated to remain shall be preserved free from injury or defacement.

\section{CONSTRUCTION REQUIREMENTS}

2 The SNLA Delegated Representative will designate all trees shrubs, plants, and other objects to remain. Any object that is designated to remain and is damaged shall be repaired or replaced as directed by the Engineer, at the Contractor's expense. Clearing and grubbing shall extend to the toe of fill or the top of cut slopes, unless otherwise designated. All surface objects, trees, stumps, roots, and other protruding obstructions not designated to remain shall be cleared and grubbed, including mowing, as required. Undisturbed stumps, roots, and nonperishable solid objects located two feet or more below subgrade or embankment slope may remain in place. In areas to be rounded at the tops of backslopes stumps shall be removed to at least two feet below the surface of the final slope line.

Except in areas to be excavated, all holes resulting from the removal of obstructions shall be backfilled with suitable material and compacted in accordance with section 02200 .

Burning of perishable material will not be permitted. They must be hauled off and disposed of in an acceptable landfill.

No material or debris shall be disposed of within the project limits without the written permission of the SNLA Delegated Representative. Material or debris that is disposed of within the project limits shall be buried to a depth of at least one foot and the surface shall be reshaped to match the adjacent ground line. The Contractor shall make all arrangements to obtain written permission from property owners for disposal locations outside the limits and view of the project. Copies of this written agreement shall be furnished to the SNLA Delegated Representative before the disposal area is used.

All cleared merchantable timber shall be removed from the project and shall become the property of the Contractor. 
The Contractor shall scalp the areas within the excavation or embankment grading limits. Mowed sod need not be removed where the embankment to be constructed is four feet or more in height. Scalping shall include the removal from the ground surface of brush, roots, sod, grass, residue of agricultural crops, sawdust, and other vegetable matter.

END OF SECTION 02110 


\section{SECTION 02200}

\section{EARTHWORK}

\section{PART 1 - GENERAL}

\subsection{SCOPE}

A. This Specification Section describes the requirements for the following:

1. The earthwork related to the following operations:

a. Dust control.

b. Excavation of materials from the site and construction of the cover system.

c. Construction of the final cover systems.

d. Subgrade preparation for the covers and permanent drainage ditches.

e. Finish grading of the site.

2. Furnishing and installing displacement monuments as shown on the Contract Drawings.

\subsection{RELATED WORK}
A. Section 01300 Descriptive Submittals
B. Section 02110 Clearing and Grubbing
C. Section 02900 Seeding.

\subsection{DEFINITIONS}

A. Excavation: Excavation is defined as excavation required to reach the lines and grades indicated on the Contract Drawings or specified herein. It shall include excavation of topsoil, silt, clay, sand, gravel, talus, soft or disintegrated rock, boulders or detached pieces of solid rock.

B. Contaminated Materials Excavation: There are no contaminated materials at this site.

C. Uncontaminated Materials Excavation: Excavation of uncontaminated materials including topsoil, silt, clay, sand, gravel, talus, soft or disintegrated rock, boulders 
and removal of detached pieces of solid rock carried out to reach lines and grades indicated on the Contract Drawings or specified herein. This excavation shall include excavations for cover, drainage ditches, and finish grading.

D. Overexcavation: Overexcavation is defined as excavation carried out beyond the lines and grades indicated on the Contract Drawings or in the Contract Specifications.

E. Percent Maximum Density: Percent maximum density is a percentage of the maximum density obtained by the test procedure presented in ASTM D698, as applicable.

F. Topsoil: Topsoil is the existing surface soil stripped to a depth of six-inches and consisting of natural, friable soil representative of productive soils in the vicinity. Topsoil shall be free of any admixture of subsoil, foreign matter, objects larger than two-inches in any dimension, toxic substances, and any material or substance that may be harmful to plant growth. This soil is to be excavated, stockpiled, and eventually refilled as the top six-inches of all disturbed areas the topsoil was originally removed from.

G. Subgrade Preparation: Subgrade preparation includes fine grading and compaction of excavations for the landfill cover systems including drainage ditches, backfills, and cover upon which bedding materials, riprap, or other features are to be constructed.

H. Cover: Cover shall consist of multiple layers of varied materials placed to form test plots as shown on the Contract Drawings.

\subsection{APPLICABLE PUBLICATIONS}

A. The Publications listed below form a part of this Specification to the extent referenced. The Publications are referred to in the text by the basic designation only:

1. American Society for Testing and Materials (ASTM):

C33-85 Specifications for Concrete Aggregates

D422-63 Method for Particle-Size Analysis of Soils

D698-91 Test methods for Moisture-Density Relations of Soils and SoilAggregate Mixtures Using $5.5 \mathrm{lb}$. (2.49-kg) Rammer and 12-in. (305-mm) Drop 
D1140-92 Test Method for Amount of Material in Soils Finer than the No. 200 (75-um) Sieve

D1556-90 Test method for Density of Soil in Place by the Sand-Cone Method

D2216-80 Test Method for Laboratory Determination of Water (Moisture) Content of Soil, Rock, and Soil-Aggregate Mixtures

D2487-93 Test Method for Classification of Soils for Engineering Purposes

D2922-91 Test Methods for Density of Soil and Soil-Aggregate in Place by Nuclear methods (Shallow Depth)

D4318-93 Test Method for Liquid Limit, Plastic Limit, and Plasticity Index of Soils

\subsection{QUALITY ASSURANCE}

A. The Contractor is responsible for meeting all requirements set forth in the contract documents. The SNLA Delegated Representative will take soil samples and perform moisture-density, gradation and other tests to ascertain that the work is being performed in compliance with these Specifications. Samples may be taken at the place of excavation, stockpiles, or on the fill itself. The SNLA Delegated Representative will conduct the density and other tests on the fill and related laboratory testing as frequently as the SNLA Delegated Representative considers necessary. The Contractor shall remove surface material and render assistance as necessary to enable sampling and testing.

B. Methods of Sampling and Testing:

1. In-Place Density: ASTM D1556 or D2922

2. Liquid Limit, Plastic Limit and Plasticity Index: ASTM D4318

3. Particle Size Analysis: ASTM D422

4. Percentage Passing No. 200 Sieve: ASTM D1 140

5. Moisture Content: ASTM D2216, D2922

6. Laboratory Moisture-Density Relations: ASTM D698

7. Soil Classification: ASTM D2487 
C. Suitability of Materials: The suitability of all materials for foundations and backfill will be approved by the SNLA Delegated Representative. Fill soil will be approved material from on-site cut excavations.

D. The SNLA Delegated Representative may direct that inspection trenches or test pits be cut into fills to determine that the Specifications have been met. Such trenches or pits will be of limited depth and size, and shall be backfilled with the material excavated therefrom, or other fill material meeting the requirements for the zones cut into. Backfill shall be compacted to a density at least equal to that of the contiguous fill.

E. Inspection trenches or test pits, and the refilling of the same, shall be at the Contractor's expense.

\section{PART 2 - PRODUCTS}

\section{$2.1 \quad$ FILL SOILS}

A. General:

1. Fill soil materials shall be obtained from on-site cut excavations. Sand and gravel shall be purchased off-site and hauled on-site.

2. The Contractor shall make his own determination of any processing that may be required, and shall perform testing as required to meet the Specifications for the various construction materials.

3. The SNLA Delegated Representative shall be granted access to each proposed source to collect samples for testing. The SNLA Delegated Representative may perform additional tests to determine if the materials meet the requirements specified herein.

4. Approval will be based on evidence of compliance with the requirements specified herein.

B. Gradations: Gradations specified shall be as determined after delivery to the site or after cut has been made and material is ready to be refilled.

C. Fill Materials (Soil) Requirements:

1. General: Fill materials for general fill shall conform to the following requirements: 
a. All materials except peat (PT), organic clay or organic silt ( $\mathrm{OL}$ or $\mathrm{OH})$ as classified by ASTM D2487. and other materials free from roots, organic matter, trash, debris, frozen materials, and stones generally not larger than $3 / 4$ inch in diameter. A sieve is to be used to ensure compliance with this maximum particle size constraint.

b. Fill material, except for demolition debris and except for clearing and stripping debris, shall not contain any visible organic material or other deleterious substances.

c. All soils used in the landfill cover test plots shall be obtained from on-site cut excavations. It is the contractor's responsibility to verify cut excavations are adequate to supply enough fill soil.

2. Soil requirements specific to each respective landfill cover test plot are as follows:

\section{Plot 4:}

\section{Topsoil Layer}

This soil is to be topsoil refilled from on-site cut excavations. This layer is to have a minimum final thickness of not less than 12 inches. This soil is not to be compacted other than that received due to equipment traffic during placement. The surface is to be scarified prior to seeding. Barrier Soil Layer

This soil is to be obtained from on-site cut excavations. It is to have a minimum thickness of 18 inches. It is to be installed lifts no thicker than 6 inches. Each lift must be scarified to a depth of 1 to 2 inches prior to placement of the next lift if the next lift is the same material. The soil is to be compacted dry of the optimum molding water content. For acceptable soil dry unit weight and water contents refer to Figure 2. The summary of Figure 2 is that compaction based on the Standard Proctor method (ASTM D698) is to be a minimum of $95 \%$ of maximum dry density but not to exceed $110 \%$ of maximum dry density. Consequently, the acceptable gravimetric soil water content range will be between $6 \%$ and $10 \%$ at $100 \%$ of maximum dry density. The acceptable water contents vary depending on the dry densities of the soil as shown in Figure 2. Both the dry density and moisture content of the soil must fall within the acceptable area shown in Figure 2. Compliance with these Specifications will be determined by the SNLA Delegated Representative.

\section{Plot 5:}

Vegetation Layer

This layer is to have a minimum thickness of 6 inches. This layer is to be topsoil obtained from on-site excavations mixed with pea gravel. The 
mixing method used to mix the topsoil and pea gravel must be submitted and approved prior to mixing. This method must ensure complete mixing of the soil and pea gravel. The pea gravel must adhere to the particle size distribution shown in Figure 3. The ratio of gravel to topsoil is to be $25 \%$ by weight $(0.25=$ dry weight of pea gravel / loose dry weight of topsoil).

\section{Cover Soil Layer}

This layer is to have a minimum thickness of 24 inches. This soil is to be obtained from on-site cut excavations. The soil is to be placed so that density approximately equates in-situ density of the soil prior to excavation (dry density between $100 \mathrm{pcf}$ and $110 \mathrm{pcf}$ ). The soil is to be placed dry of optimum molding water content, therefore the gravimetric water content of the soil must be within the acceptable range shown in Figure 5. Compliance with these Specifications will be determined by the SNLA Delegated Representative.

\section{Plot 6:}

The soil for landfill cover test plot 6 shall be obtained from on-site cut excavations. The overall thickness of the cover is to have a minimum thickness of 36 inches. The bottom 30 inches of soil shall be placed in lifts not to exceed a 6 inch compacted thickness. Each lift is to be compacted based on the Standard Proctor method (ASTM D698) to between $95 \%$ and $110 \%$ of maximum dry density. For acceptable soil dry unit weights and molding water contents refer to Figure 2. Each lift is to be scarified to a depth of 1 to 2 inches prior to the placement of the subsequent lift. The top 6 inches shall be loosely placed (topsoil) to allow for vegetation planting and seeding. If this top 6 inches is compacted too densely by equipment traffic, it is to be scarified at the discretion of the Sandia Delegated Representative.

D. Sand requirements specific to each respective landfill cover test plot are as follows:

\section{Plot 4}

Sand Upper Drainage Layer 1:

The sand used in this layer is to purchased off-site and trucked on-site. This layer shall have a minimum thickness of 3 inches. The sand shall have a saturated hydraulic conductivity greater than $0.01 \mathrm{~cm} / \mathrm{sec}$. It shall also meet the grain size distribution requirements shown in Figure 3. The General Contractor must provide a submittal showing that the sand to be used meets the grain size distribution and hydraulic conductivity requirements.

\section{Lower Drainage Layer:}

The sand used in this layer is to purchased off-site and trucked on-site. This layer shall have a minimum thickness of 12 inches. The sand shall have a saturated 
hydraulic conductivity greater than $0.01 \mathrm{~cm} / \mathrm{sec}$. It shall also meet the grain size distribution requirements shown in Figure 4. The General Contractor must provide a submittal showing that the sand to be used meets the grain size distribution-and hydraulic conductivity requirements.

\section{Plot 5}

\section{Interface Layer}

The sand used in this layer is to purchased off-site and trucked on-site. This layer shall have a minimum thickness of 6 inches. The sand shall have a saturated hydraulic conductivity greater than $0.01 \mathrm{~cm} / \mathrm{sec}$. It shall also meet the grain size distribution requirements for sand shown in Figure 3. The General Contractor must provide a submittal showing that the sand to be used meets the grain size distribution and hydraulic conductivity requirements.

E. Gravel requirements specific to each respective landfill cover test plot are as follows:

\section{Plot 4}

Pea Gravel Upper Drainage Layer 2:

The pea gravel used in this layer is to purchased off-site and trucked on-site. This layer shall have a minimum thickness of 9 inches. The gravel shall have a saturated hydraulic conductivity greater than $0.1 \mathrm{~cm} / \mathrm{sec}$. It shall also meet the grain size distribution requirements shown for gravel in Figure 3. The General Contractor must provide a submittal showing that the sand to be used meets the grain size distribution and hydraulic conductivity requirements.

\section{Plot 5}

\section{Sublayer}

The pea gravel used in this layer is to purchased off-site and trucked on-site. This layer shall have a minimum thickness of 6 inches. The sand shall have a saturated hydraulic conductivity greater than $0.1 \mathrm{~cm} / \mathrm{sec}$. It shall also meet the grain size distribution requirements shown for gravel in Figure 3. The General Contractor must provide a submittal showing that the sand to be used meets the grain size distribution and hydraulic conductivity requirements. 


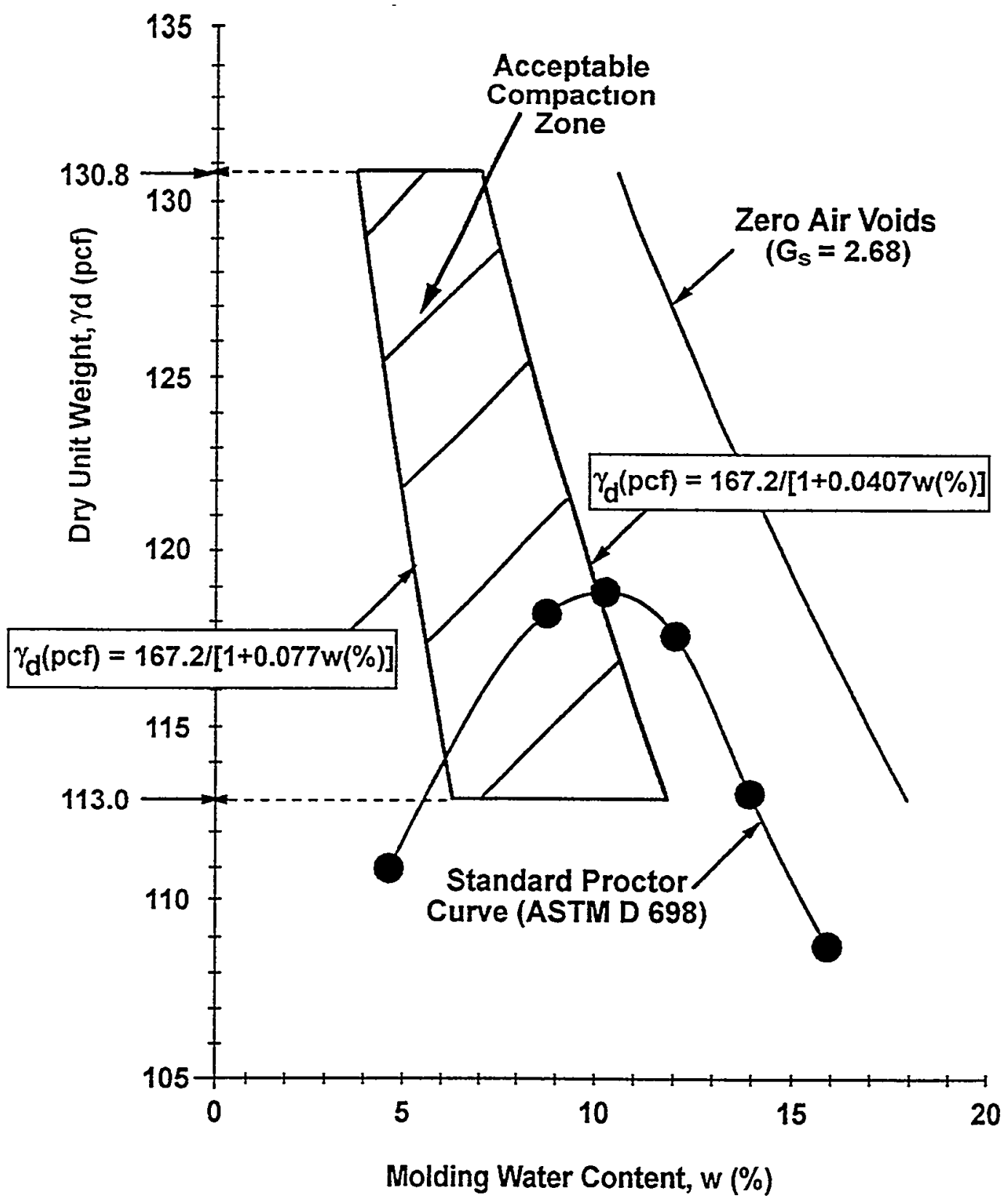

Figure 2 - Acceptable Compaction Zone for Barrier Soil Layer for Dry-Side Compaction

(Plot 4: Barrier Soil Layer; Plot 6: Compacted Native Soil Layer) 


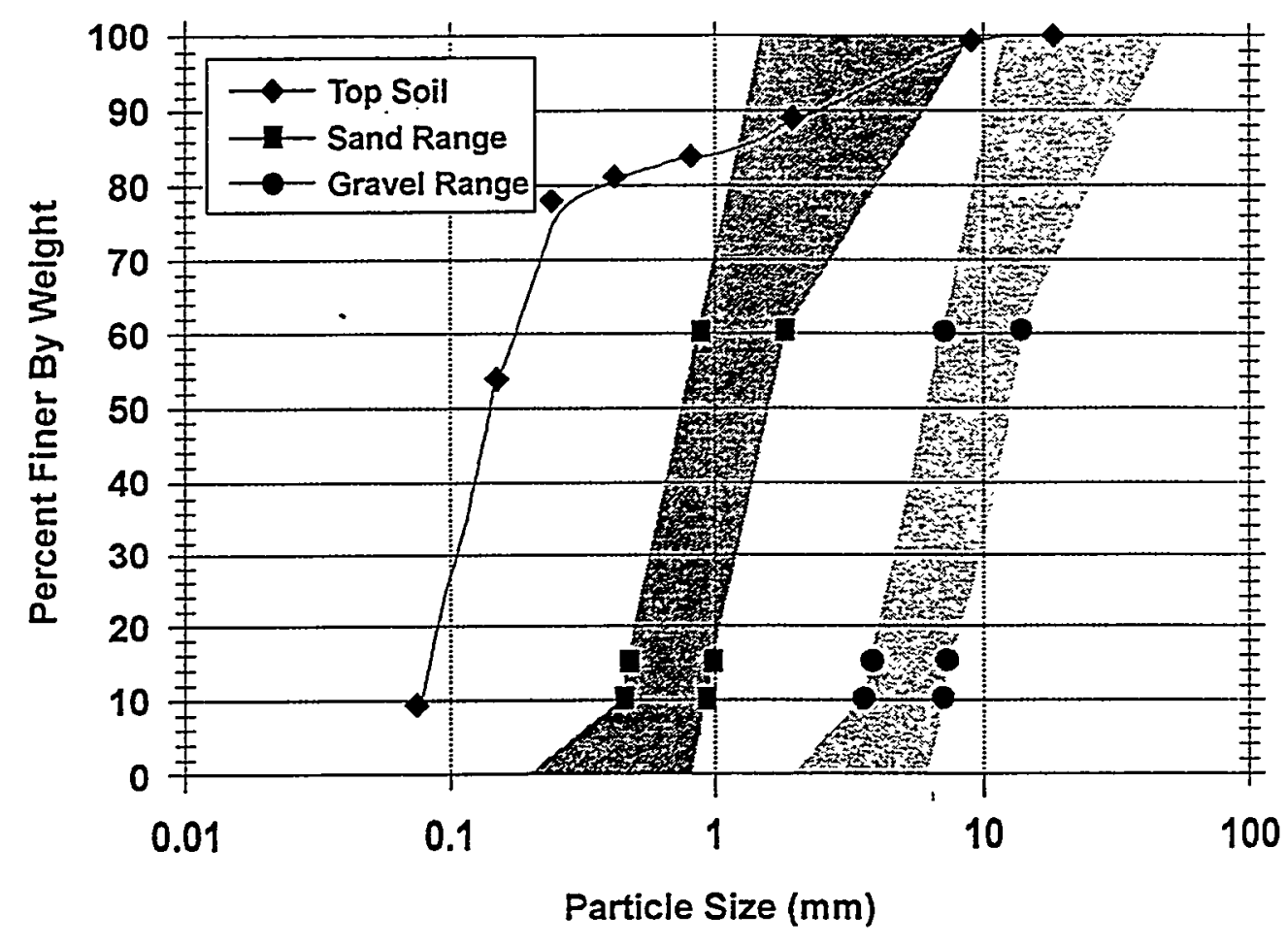

Figure 3 - Acceptable Ranges for Particle Size Distribution of Sand and Gravel (plot 4: sand - upper drainage layer 1, pea gravel - upper drainage layer 1; plot 5: pea gravel/topsoil mix, sand interface layer, pea gravel sub layer) 


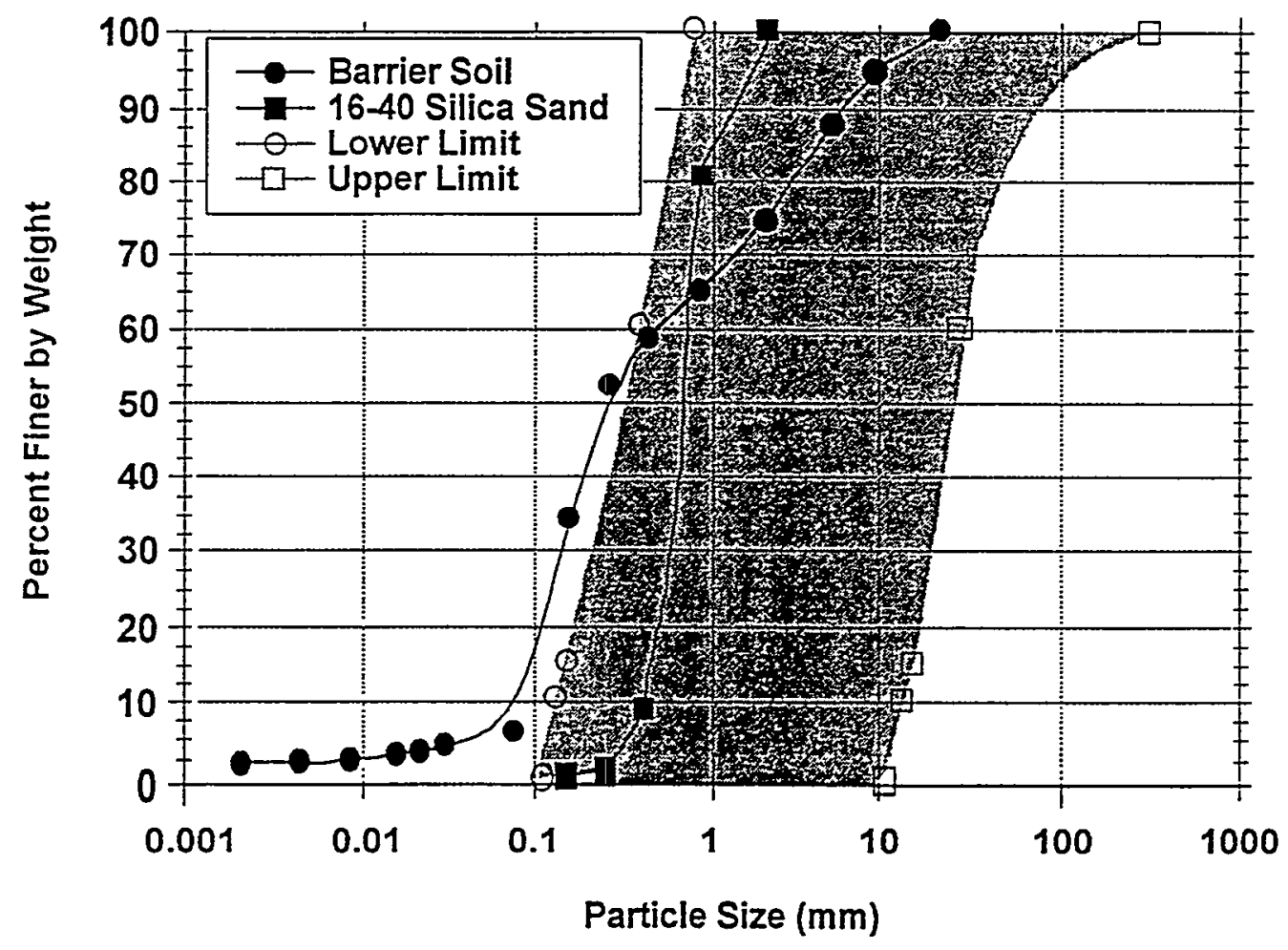

Figure 4 - Acceptable Ranges for Particle-Size Distribution of sand (Plot 4: sand - lower drainage layer) 


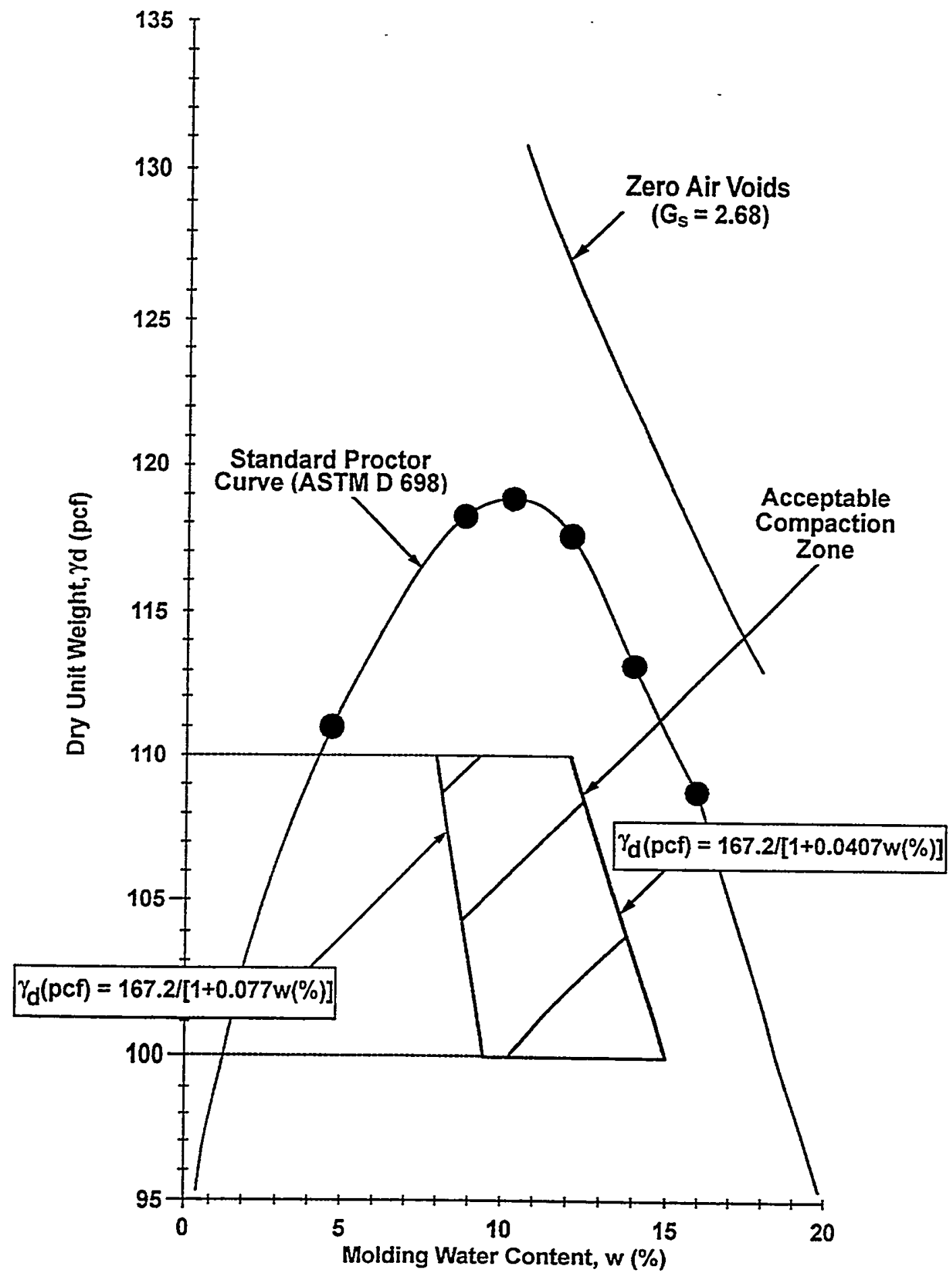

Figure 5 - Acceptable Compaction Zone for Soil Layer for Dry-Side Compaction (Plot 5: Native Soil Layer) 


\subsection{EQUIPMENT}

All equipment to be used on the project must be submitted to and approved by the SNLA Designated Representative.

A. Compaction equipment:

1. Kneading -- Compactor weight in the range of 30,000 to $40,000 \mathrm{lbs}$. (feet must be substantially less than the loose lift thickness so as not to damage any instrumentation placed in prior lift).

2. Smooth Roll -- Compactor weight in the range of 30,000 to $40,000 \mathrm{lbs}$..

\section{PART 3 - EXECUTION}

\subsection{DUST CONTROL}

A. Dust control shall consist of furnishing water supply, required equipment, additives, accessories and incidentals, and applying water during the completion of the Contract, as required by the SNLA Delegated Representative . A minimum moisture content of four percent shall be maintained in the upper 6-inches of the roadbeds at all times.

B. Water shall be applied by means of pressure-type distributors or pipe lines equipped with a spray system or hoses with nozzles that will insure a uniform application of water.

C. All equipment used for the application of water shall be equipped with a positive means of shut-off.

D. Unless otherwise permitted by the SNLA Delegated Representative or unless all the water is applied by means of pipe lines, at least one mobile unit with a minimum capacity of 5,000 gallons shall be available for applying water on the project at all times.

E. If the Contractor uses chemical additives in water, furnishing and applying the additives shall be at the Contractor's expense.

F. The use, location of application, and the amount and type of additives proposed for use by the Contractor must be submitted to and approved by the SNLA Delegated Representative prior to their use.

\subsection{EXCAVATION}

A. Preparation: 
1. Required lines, levels, contours and datum shall be identified before the start of excavation.

B. General:

1. At all times during excavation, the Contractor shall conduct his operations in such a manner as to prevent free standing water and contamination of uncontaminated materials. The Contractor shall, as a minimum, maintain positive grade to safeguard against such problems.

2. The Contractor shall perform required excavation to the lines and grades indicated on the Contract Drawings. He shall remove all excavated material from the excavation site and dispose of it.

3. Unsuitable or low density subgrade material not readily capable of in-place compaction shall be excavated as directed by the SNLA delegated representative and disposed of as a topsoil.

4. Adequate working space for safety of personnel shall be provided within the limits of the excavation.

5. All unstable material, large stones, debris and incompressible soils shall be removed from bottoms of the excavation to a minimum depth of 12-inches.

6. Except as otherwise noted, care shall be exercised to preserve the material below and beyond the lines of all excavation. Where excavation is carried below grade, the Contractor shall backfill to the required grade or to indicated invert grade, as specified, and recompact the backfill to meet the existing conditions.

7. Excavation for the convenience of the Contractor shall conform to the limits approved by the SNLA Delegated Representative and shall be at no additional expense to SNLA.

8. Where practicable, suitable materials removed from excavation shall be used as fill or backfill.

C. Materials Excavation:

1. General: Materials excavation shall include excavations of materials from the various areas of the site. The excavated materials shall be used as fill in various areas of the site including the construction of berms, dikes, general fill, roadway fill, structure fill, backfill, and fill for the final grading of the site, as required. Excavated material may be stockpiled for later use. 


\section{Drainage Swales Excavation:}

a. General: Swales shall be cut accurately to the cross sections and grades. where indicated. All roots, stumps, rock, and foreign matter in the sides and bottom of swales shall be trimmed and dressed or removed to conform to the slope, grade, and shape of section indicated. Care shall be taken not to excavate ditches below the grades indicated. Excessive ditch excavation shall be backfilled to grade with satisfactory, thoroughly compacted material. Ditches shall be maintained until final acceptance of the work. Except the north and east swale excavation may be extended to provide adequate fill for the cover construction.

b. Permanent Drainage Swales:

Ditches shall be excavated true to line and grade. Any erosion which occurs to ditch excavation before placing erosion protection materials shall be repaired with compacted backfill. All such repairs shall be at Contractor's expense.

The top six-inches of the subgrade shall be compacted as specified in Article 3.4 below. After compaction has been completed, finish grading shall be done in such a manner that the sideslopes are rendered smooth surfaces. All rocks, brush, roots, large clods, and other objects shall be removed.

c. The area to be excavated must be cleared and grubbed in accordance with section 02110. All topsoil will be stockpiled for later use.

\section{Embankment Materials:}

1. Materials excavated from the site, including excavations for trenches, and drainage ditches shall be used as fill for construction of various features including site grading, or stockpiled for later use, or wasted in the spoil area, as specified in this Section and is required by the SNLA Delegated Representative.

2. Where used in fills, such material shall be transported directly from the excavation and placed in its final position in such fills whenever possible. If required by the Contractor's schedule, the material may be placed temporarily in stockpiles at approved locations. Material in stockpile shall be protected from contamination of any.kind that would render it unsuitable for use in fills.

E. Unsatisfactory or Excess Materials: Unsatisfactory and excess excavated uncontaminated material generated during the Work and not approved for use in 
the Work shall be disposed of in the designated stockpile/spoil areas as directed by the SNLA Delegated Representative.

F. Garbage, refuse, debris, oil, and any waste material which is generated by the Contractor and is harmful to the environment or offensive to the area shall be removed from the job site and disposed of offsite in a manner approved by the authority having jurisdiction over the disposal site.

G. All operations in the stockpile/spoil areas throughout the Work shall be in strict conformity with the requirements of this Section.

\subsection{FILI CONSTRUCTION}

A. General Requirements:

1. Fill materials shall be placed and compacted to the lines and grades shown on the Contract Drawings .

2. If any portion of the materials does not meet the specified requirements, the Contractor shall remove such material and replace it with fill materials meeting the specification at no additional cost.

B. Placing Requirements:

1. Prepared subgrade: Prior to placement of Materials, the in-place density of the top six-inches of the area receiving the fill shall be at least 95 percent of maximum dry density as determined by ASTM D698. If necessary, the surface shall be plowed, borrowed materials mixed, materials added or removed and compacted to obtain the specified densities.

2. Fill materials may require moisture conditioning (wetting or drying) prior to compaction.

3. Fill materials shall be placed in continuous and approximately horizontal layers for their full length and width unless otherwise specified.

4. The method of dumping and spreading the materials shall ensure uniform distribution of the material.

5. The loose thickness of each layer of materials shall not be greater than that required to achieve the required compaction, and in no case shall exceed 12inches for the fill and nine-inches for the barrier materials. These thicknesses are dependent on the compaction equipment to be used. The kneading foot length must be substantially less than the loose lift thickness so as not to damage any instrumentation placed in prior lift. 
6. The Contractor shall place material to a grade of one percent or steeper to preclude the ponding of water. No fill shall be placed on any area where ponding has been allowed to occur. Where ponding has occurred and the fill material is required to be placed, the water shall be removed and the subgrade reprocessed to 95 percent of ASTM D698.

7. Materials shall not be placed on frozen subgrade or embankment material foundations, nor shall frozen material be used as fill.

C. Compaction / Remolding Requirements:

1. Each layer of shaping fill materials leading to the final prepared subgrade shall be compacted to a minimum of 95 percent of maximum dry density as determined by ASTM D698.

2. During compaction the moisture content of shaping fill material shall be maintained to achieve the specified density. Uniform moisture distribution shall be obtained by disking, blading, or other methods approved by the SNLA Delegated Representative prior to compaction of a layer.

3. All lift surfaces must maintain a uniform slope.

4. If the rolled surface of any layer of the fill in place is too wet for proper compaction of the layer of fill material to be placed thereon, it shall be removed, allowed to dry or worked with harrow, scarifier, or other suitable equipment to reduce the water content to the required amount, and then recompacted before the next succeeding layer of fill is placed.

5. Fill placed at densities lower than the specified minimum density or at moisture contents outside the specified acceptable range of moisture content shall be reworked to meet the density and moisture requirements or removed and replaced by acceptable fill compacted to meet these requirements.

D. Field Quality Control:

1. General: The SNLA Delegated Representative will take samples and perform tests throughout the construction period, and the Contractor shall cooperate in providing access for the SNLA Delegated Representative to areas where testing is to be performed and shall schedule his placing to avoid interference with the testing operations.

2. Tests: The contractor will be responsible for performing his own tests to ensure compliance with all contract provisions. Copies of these tests are to 
provided to the Sandia Delegated Representative. The SNLA Delegated Representative will also perform tests. The Contractor must make allowances for these tests at no additional cost to Sandia. Refer to the ALCD QA plan for approximate number and type of tests to be performed by SNLA personnel.

\subsection{Subgrade Preparation For Permanent Drainage Ditches.}

The top six-inches of the subgrade of each permanent drainage ditch shall be compacted to a minimum of 95 percent of maximum density as determined by ASTM D698. After compaction has been completed, fine finishing shall be done in such a manner that the side-slopes are smooth surfaces. All rocks, brush, roots, large clods, and other objects shall be removed before placement of the bedding materials.

3.5 Subgrade Preparation of Infiltration Barrier for Placement of Geomembrane.

Surface shall be smooth rolled. No rocks, clods, or other form of debris shall be exposed larger than one-half inch in diameter.

END OF SECTION 02200 


\section{SECTION 02210}

\section{RIPRAP}

\section{PART 1 - GENERAL}

\subsection{SCOPE}

This Specification Section describes the requirements for furnishing and placing rip rap and bedding materials for the cover and permanent drainage ditches.

\subsection{RELATED WORK}
A. Section 01300 Descriptive Submittals.
B. Section 02200 Earthwork: Subgrade preparations.

\subsection{APPLICABLE PUBLICATIONS}

A. The publications listed below form a part of this Specification to the extent referenced. The publications are referred to in the text by the basic designation only:

1. American Society for Testing and Materials (ASTM):

C88-83 Test Method for Soundness of Aggregates by Use of Sodium Sulfate or Magnesium Sulfate

C127-84 Test Method for Specific Gravity and Absorption of Coarse Aggregate

C131-81 Resistance to Degradation of Small-Size Coarse Aggregate by Abrasion and Impact in the Los Angeles Machine

C295-85 Practice for Petrographic Examination of Aggregates for concrete

C535-81 Test Method for Resistance to Degradation of Large-Size Coarse Aggregate by Abrasion and Impact in the Los Angeles Machine

PART 2 - Products

\subsection{MATERIALS}


A. Material Sources: Erosion protection materials shall be obtained from sources approved by the SNLA Delegated Representative. Every change in source of materials shall require approval from the SNLA Delegated Representative.

1. Approval of the source as a borrow area does not mean that all materials excavated will meet the requirements of this Specification. Processing or selective quarrying may be necessary to meet the quality requirements of this Section.

2. The basis for approval of the sources proposed by the Contractor shall be a site inspection report by an engineering geologist or geotechnical engineer which will include, as a minimum, an evaluation of soundness, hardness, and durability for three samples representative of the proposed source.

B. All materials shall be free from radioactive or other contamination.

C. Rip rap materials:

1. Individual pieces shall be dense, sound, resistant to abrasion, and shall be free from cracks, seams, and other defects as shown in the petrographic examination.

2. The shape of at least seventy percent of the material, by weight, shall be such that the minimum dimension is not less than one third of the maximum dimension. No crushed stones may be used because the sharp edges may damage the liners. Preferably rounded to surrounded stones will be used.

3. Quality:

Tests

Specific Gravity

(Saturated Surface

Dry Basis)

Absorption

Soundness

Abrasion
Designation

ASTM C127

ASTM C127

ASTM C88

ASTM C131
Requirements

Not more than 2.45

Not more than 1.5 percent.

$\mathrm{NaSO}_{4}$ Test: Not more than 5 percent loss of weight after 5 cycles.

Not more than 35 percent (Los Angeles Machine) loss of weight after 500 revolutions. Ratio of weight 
loss after 100 revolutions to loss after 500 revolutions shall not exceed 30 percent.

Abrasion

Petrographic
ASTM C535

ASTM C295
Not more than 50 percent (Los Angeles Machine) loss of weight after 1000 revolutions. Ratio of weight loss after 200 revolutions to loss after 1000 revolutions shall not exceed 20 percent.

The Contractor shall furnish a report for review by the Engineer.

4. The riprap shall be sized as follows: maximum size stone - 8-inches, median size stone - 5-inches, minimum layer thickness - 12-inches. The size of an individual stone shall be determined by measuring its long dimension.

Gradation is to be well graded with at least $50 \%$ of the gradation between the maximum size stone and the median size stone. The remaining riprap materials shall be reasonably well graded within the following limits:

U.S. Standard Sieve Size (Square Openings) Material Size 4"

2"

$1 "$

\#4

100
Percent Passing

(by weight)

100

$30-95$

$20-50$

$0-20$

$0-5$

\section{PART 3 - EXECUTION}

\subsection{PLACEMENT AND COMPACTION}

A. Subgrade preparation (for drainage ditches, etc.) shall conform to Specification Section 02200.

B. Where the required layer thickness is 12 -inches or less, the material shall be spread in one layer.

C. The riprap will not require compaction. 
D. Riprap material shall be placed so that the larger pieces are uniformly distributed and the smaller pieces serve to fill the spaces between them to provide wellkeyed, densely placed layers of riprap of the specified thicknesses.

E. Riprap material may be placed by end-dumping and may be spread by bulldozers or other suitable equipment. The material shall not be dropped from a height exceeding two-feet above the riprap material, nor in a manner to cause fracture, displacement, or damage to the material being placed or already in place. In no case shall the riprap be dropped directly on the liners but shall be dumped on already placed riprap and then pushed into its final position .

F. Construction equipment other than spreading and placement equipment shall not be allowed to move over the placed riprap material layers.

\subsection{TOLERANCES}

A. The material layers shall be placed to the limits and thicknesses shown on the Contract Drawings within the following tolerances:

1. Top of bedding material shall be within 0.1 foot of elevations shown on the Contract Drawings.

2. The minimum in-place thickness shall not be more than 100 percent of the thickness shown.

3. The maximum in-place thickness shall not be more than 135 percent of the thickness shown.

4. Local irregularities will be permitted provided that such irregularities do not form noticeable mounds, ridges, swales, or depressions.

\subsection{FIELD QUALITY CONTROL}

A. The placement of the materials shall be monitored to ensure that the following requirements are met:

1. The correct type of material is being placed.

2. The material being placed is clean and free of unsuitable material.

3. The material is being loaded, transported, and placed in a manner which minimizes segregation. 
4. Deviations from minimum and maximum thickness shall not exceed 50 percent of the maximum rock size.

5. The material is being placed to line and grade within the tolerances and limits designated in Article 3.2 above.

B. All unsatisfactory materials shall be removed to the satisfaction of the SNLA Designated Representative and replaced with acceptable materials at no additional cost.

C. The SNLA Designated Representative may require that material which has become segregated or not placed according to the above requirements to be regraded or adjusted, using appropriate equipment, to conform with the tolerances and limits given above, at no additional cost.

END OF SECTION 02210 


\section{SECTION 02725}

\section{MANHOLES}

\section{PART 1 - GENERAL}

\subsection{DESCRIPTION OF WORK}

A. This specification shall apply to the materials and operations required for the installation of manholes.

B. The extent of the work is indicated on the Contract Drawings.

\subsection{RELATED WORK}

1. Section 01300 "Descriptive Submittals".

2. Section 02200 "Earthwork".

3. Section 03300 "Concrete".

4. Section 09900 "Painting".

\subsection{QUALITY ASSURANCE}

A. The materials and practices comprising the work shall conform to this and other referenced standard specifications.

\section{PART 2 - PRODUCTS}

\subsection{MATERIALS}

A. Cement shall conform to the following requirements:

1. Cement for use in mortar shall conform to ASTM C 91.

2. Portland cement for use in precast and cast-in-place concrete, and in masonry grout and mortar shall conform to ASTM C 150, type II, IIA, or III.

B. Block, Brick, Precast Barrels, Steps, Frames and Covers:

1. Riser Sections and Tops: Precast reinforced concrete conforming to ASTM C 478. Precast tops shall be either eccentric cone or flat slab type. Concrete 
for precast sections shall have a minimum of 4000 psi 28 -day compressive strength.

2. Riser Joints: Rubber gasket conforming to ASTM C 443, bituminous mastic gasket, or butyl rubber gasket.

3. Concrete Masonry Units: Solid precast segmental block conforming to ASTM C 139.

4. Mortar: Type M.

5. Brick: Grade MS, conforming to ASTM C 32.

6. Steps: Steel reinforced polypropylene conforming to ASTM C 478.

7. Frames and Covers: Lightweight - capable of supporting a 300-pound vertical load.. Bearing surfaces shall be ground or machined, such that the cover shall seat firmly onto the frame without rocking. Submit for approval.

\section{PART 3 - EXECUTION}

\subsection{MANHOLE CONSTRUCTION}

A. General: Manholes shall be installed at the locations and elevations indicated and as detailed on the Contract Drawings. Installed pipe invert elevations at manholes shall be as designated on the Contract Drawings.

B. Manhole Bases: Prior to manhole installations, prepare a subgrade soil

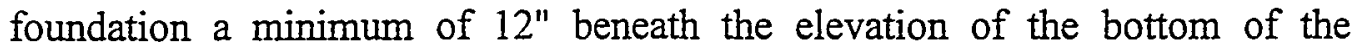
concrete manhole base and compact to $95 \%$ maximum density as determined by ASTM D698. Cast-in-place concrete for the base shall have a minimum of 3000 psi 28- day compressive strength.

C. Joints in precast riser sections and tops shall be made using gasket materials in accordance with the manufacturer's written installation instructions. These joints must be sealed watertight.

D. Precast Concrete Manhole Wall Sections:

1. Wall sections are to be precast concrete only.

2. Holes for pipe sections can be precast at designated locations. However, the preferred method is to core drill the pipe holes on-sight to allow for possible field variations in pipe locations. 
E. Pipe sections built into manhole walls shall be reinforced with grout so that the manhole and adjoining pipe sections are monolithic.

F. Manhole. Floors: Manhole floors shall be made of grout and the work shall be free of any rough comers or sudden changes in direction such that a steady uniform flow with a minimum of wave action will be provided. Changes in direction and grade will consist of the largest curve radius the manhole diameter will permit. Free vertical drop from any branch or service line shall not exceed one half the mainline pipe diameter measured from the mainline upstream invert.

G. Manhole steps shall be cast-in-place or driven into precast or site-drilled holes. Steps shall be installed not more than 12 " apart vertically on the interior wall directly beneath the manhole cover according to ASTM C 478.

H. Manhole frames and covers shall be installed as detailed and adjusted to required elevation by building up courses of manhole brick on full beds of mortar with "push joints" or by using precast concrete adjustment rings set in full beds of mortar.

I. Fill all lifting holes and other voids inside and outside with non-shrink grout. The inside of the manhole shall be cleaned of all loose mortar, framing materials and other debris.

J. Bituminous waterproof coating shall be applied to the exterior surface of the manhole. When designated on the Contract Drawings, the interior of the manhole shall be coated with a corrosion resistant material.

$\mathrm{K}$. The manholes are to be finish constructed to be watertight.

\section{END OF SECTION 02725}




\section{SECTION 02776}

\section{GEOMEMBRANE}

1.0 Related Work.

A. Section 01300 Descriptive Submittals.

B. Section 02200 Earthwork: Subgrade Preparation.

2.0 Material

2.1 The geomembrane is to be a 40 mil linear low density polyethylene membrane meeting the following properties:

\begin{tabular}{|c|c|c|c|c|}
\hline Resin Properties & Method & Units & Minimum & Typical \\
\hline Melt Flow Index & ASTMD 1238 & $\mathrm{~g} / 10 \min$ & 1.3 & 0.94 \\
\hline Resin Density & & $\mathrm{g} / \mathrm{cm}^{3}$ & 0.925 & 0.918 \\
\hline Sheet Properties & Method & Units & Minimum & Typical \\
\hline Thickness & $\begin{array}{l}\text { ASTM D } 751 \\
\text { NSF mod. }\end{array}$ & & & \\
\hline Average & & mils & 40.0 & 41.5 \\
\hline Individual & & mils & 38.0 & 40.3 \\
\hline Density & ASTM D 1505 & $\mathrm{~g} / \mathrm{cm}^{3}$ & 0.938 & 0.929 \\
\hline Carbon Black Content & $\begin{array}{l}\text { ASTMD } 3015 \\
\text { NSF mod. }\end{array}$ & rating & $\mathrm{A} 1, \mathrm{~A} 2, \mathrm{~B} 1$ & Al \\
\hline Tensile Properties & ASTM D 638 & & & \\
\hline \multirow[t]{2}{*}{ Stress at Yield } & & psi & 1640 & 1720 \\
\hline & & ppi & 66 & 71 \\
\hline \multirow[t]{2}{*}{ Stress at Break } & & psi & 3800 & 4600 \\
\hline & & ppi & 152 & 191 \\
\hline Strain at Yield & $\begin{array}{l}1.3 " \text { gage length } \\
\text { (NSF) }\end{array}$ & percent & 15.0 & 20.0 \\
\hline \multirow[t]{2}{*}{ Strain at Break } & $\begin{array}{l}2.0 \text { " gage or } \\
\text { extensiometer }\end{array}$ & percent & 750 & 880 \\
\hline & $\begin{array}{l}2.5 " \text { gage length } \\
\text { (NSF) }\end{array}$ & percent & 600 & 700 \\
\hline \multirow[t]{2}{*}{ Tear Resistance } & ASTM D 1004 & ppi & 590 & 625 \\
\hline & & lbs. & 24 & 26 \\
\hline \multirow[t]{2}{*}{ Puncture Resistance } & FTMS 101C, & ppi & 1450 & 1500 \\
\hline & 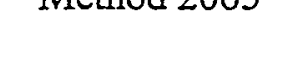 & lbs & 58 & 62 \\
\hline
\end{tabular}




\subsection{Material Delivery}

The SNLA Delegated Representative will be present, whenever possible, to observe and assist in material delivery and unloading on site. The SNLA Delegated Representative is to note any materials received in a damaged state and note any material damaged during unloading. Upon mobilization to site, a SNLA Delegated Representative shall:

- Verify the equipment used on site is adequate and does not risk damage to the geomembrane or other materials.

- Mark rolls or portions of rolls which appear damaged.

- Verify that storage of materials ensures adequate protection against dirt, theft, vandalism, and passage of vehicles.

- Ensure that rolls are properly labeled and that labeling corresponds with Quality Control documentation and Shipping Bills of Lading.

- Assist SNLA Delegated Representative, if present, with material sampling or record keeping.

- Complete roll numbers, date, bill of lading number, roll size, and any damage noted will be logged on the Material Delivery Checklist.

\section{GEOMEMBRANE INSTALLATION}

\subsection{Earthwork}

3.1.1 The General and/or Earthwork Contractor shall be responsible for preparing and maintaining the subgrade in a condition suitable for installation of the liner unless specifically agreed otherwise. Refer to section 02200 .

\subsubsection{The following general minimum guidelines shall be followed.}

3.1.2.1 Surfaces to be lined shall be smooth and free of debris, roots, and angular or sharp rocks. All fill shall consist of well-graded material, free of organics, trash, clay balls, or other deleterious material that may cause damage to the geomembrane. The upper six inches of the finished subgrade shall not contain stones or debris larger than onehalf inch. The subgrade shall be compacted in accordance with design specifications, but in no event less than is required to provide a firm, unyielding foundation sufficient to permit the movement of vehicles and welding equipment over the subgrade without causing rutting or other deleterious effects. The subgrade shall have no sudden sharp or abrupt changes in grade.

3.1.2.2 The Earthwork Contractor shall protect the subgrade from desiccation, flooding, and freezing. Protection, if required, may 
consist of a thin plastic protective cover (or other material as approved by the engineer) installed over the completed subgrade until such time as the placement of geomembrane liner begins. Subgrades found to have desiccation cracks greater than one-half inch in width or depth, or which exhibit swelling, heaving, or other similar conditions shall be replaced or reworked by the General and/or Earthwork Contractor to remove these defects.

\subsubsection{Surface Acceptance. Upon request, the manufacturer's representative} will provide the Owner's and/or Contractor's Representatives with a written acceptance of the surface to be lined. This acceptance will be limited to the amount of area that the contractor is capable of lining during a particular work shift. Direction and control of any subsequent repairs to the subgrade, including the subgrade surface, shall remain the responsibility of the Earthwork Contractor.

\subsection{Preparation for Geomembrane Deployment}

\subsubsection{Panel Layout}

Prior to commencement of liner deployment, layout drawings shall be produced to indicate the panel configuration and general location of field-seams for the project. The layout must be submitted to and approved by the SNLA Delegated Representative.

\subsubsection{Identification}

Each panel used for the installation shall be given a numeric or alpha-numeric identifier. This panel identification number shall be related on the Panel Placement Form to a manufacturing roll number that identifies the resin type, batch number, and date of manufacture.

\subsection{Field Panel Placement}

\subsubsection{Weather Conditions}

Geomembrane deployment will generally not be done during any precipitation, in the presence of excessive moisture (i.e. fog, dew), in an area of standing water, or during high winds.

\subsubsection{Location}

The Contractor is to install field panels as indicated on the approved layout drawing. If the panels are deployed in a location other than that indicated on the approved layout drawings they will be removed and replaced in correct 
locations. If any materials are damaged during this process, they will be replaced at the contractor's expense. The revised location shall be noted in the field on a record "as-built" drawing which will be modified at the completion of the project to reflect actual panel locations. Record drawings will be maintained and submitted by the Contractor.

\subsubsection{Documentation of Panel Placement}

Information relating to geomembrane panel placement including date, time, panel number, and panel dimensions will be maintained on the Panel Placement Form.

3.3.3.1 If a portion of a roll is set aside to be used at another time, the roll number will be written on the remainder of the roll in several places.

\subsubsection{Method of Deployment}

3.3.4.1 The method and equipment used to deploy the panels must not damage the geomembrane or the supporting subgrade surface.

3.3.4.2 No personnel working on the geomembrane will wear shoes that can damage the geomembrane or engage in actions which could result in damage to the geomembrane.

3.3.4.3 Adequate temporary loading and/or anchoring, (i.e. sandbags, tires), which will not damage the geomembrane, will be placed to prevent uplift of the geomembrane by wind.

3.3.4.4 The geomembrane will be deployed in a manner to minimize wrinkles.

3.3.5 Any area of a panel seriously damaged (torn, twisted, or crimped) will be marked and repaired in accordance with Paragraph 5.3 of this document.

\subsection{Geomembrane Field Seaming}

\subsubsection{General Requirements}

\subsubsection{Layout}

In general, seams shall be oriented parallel to the slope, i.e., oriented along, not across the slope. Whenever possible, horizontal seams should be located on the base of the cell, not less than five feet from the toe of the slope. Each seam made in the field shall be numbered and indicated on the record drawings. Seaming information to include seam number, 
welder ID, machine number, temperature setting, and weather conditions will be maintained on a Panel Seaming Form.

\subsubsection{Personnel}

All personnel performing seaming operations shall be trained in the operation of the specific seaming equipment being used and will qualify by successfully welding a test seam as described in Paragraph 3.5.3. The project foreman will provide direct supervision of the seaming operations.

\subsubsection{Equipment}

\subsection{Fusion Welding}

Fusion Welding consists of placing a heated wedge, mounted on a self propelled vehicular unit, between two overlapped sheets such that the surface of both sheets are heated above the polyethylene's melting point. After being heated by the wedge, the overlapped panels pass through a set of pre-set pressure wheels which compress the two panels together to form the weld. The fusion welder is equipped with a device which continuously monitors the temperature of the wedge.

\subsection{Extrusion Fillet Welding}

Extrusion fillet welding consists of introducing a ribbon of molten resin along the edge of the overlap of the two geomembrane sheets to be welded. A hot-air preheat and the addition of molten polymer causes some of the material of each sheet to be liquefied resulting in a homogeneous bond between the molten weld bead and the surfaces of the overlapped sheets. The extrusion welder is equipped with gauges giving the temperature in the apparatus and a numerical setting for the pre-heating unit.

\subsubsection{Weather Conditions}

Many factors, such as the geomembrane temperature, humidity, wind, precipitation, etc. , can effect the integrity of field seams and must be taken into account when deciding whether or not seaming should proceed. Test seams, as described in Paragraph 3.5.3, are required prior to daily production seaming to determine if the weather conditions will effect the Contractor's ability to produce quality seams. Additional non- 
destructive and destructive testing of production seams substantiate the decision made by the Project Superintendent to seam on any given day.

\subsubsection{Seam Preparation}

\subsubsection{Fusion Welding}

3.4.2.1.1 Overlap the panels of Geomembrane approximately four to six inches prior to welding.

3.4.2.1.2 Clean the seam area prior to seaming to assure the area is clean and free of moisture, dust, dirt or debris of any kind. No grinding is required for fusion welding.

3.4.2.1.3 Adjust the panels so that seams are aligned with the fewest possible number of wrinkles and "fish mouths".

3.4.2.1.4 A movable protective layer may be used, at the discretion of the Contractor, directly below the overlap of geomembrane that is to be seamed to prevent build-up of dirt or moisture between the panels.

\subsubsection{Extrusion Fillet Welding}

3.4.2.2.1 Whenever possible, extrusion welded seams will be prebeveled prior to heat-tacking into place.

3.4.2.2.2 Overlap the panels of geomembrane a minimum of three inches.

3.4.2.2.3 Using a hot-air device, temporarily bond the panels of geomembrane to be welded, taking care not to damage the geomembrane.

3.4.2.2.4 Clean the seam area prior to seaming to assure the area is clean and free of moisture, dust, dirt, and debris of any kind.

3.4.2.2.5 Grind seam overlap prior to welding within one hour of the welding operation in a manner that does not damage the geomembrane. Grind marks should be covered with extrudate whenever possible. In all cases grinding should not extend more than one-quarter inch past the edge of the area covered by the extrudate during welding. 
3.4.2.2.6 Purge the extruder prior to beginning the seam to remove all heat-degraded extrudate from the barrel. The purged extrudate will be placed on scrap material so as to prevent contact with installed geomembrane.

\subsection{Keep welding rod clean and dry.}

\subsubsection{Trial Welds}

Trial welds shall be conducted by Welding Technicians prior to each seaming period, every five hours, as weather conditions dictate, or as requested by the SNLA Delegated Representative if welding problems are suspected. All trial welds will be conducted under the same conditions as will be encountered during actual seaming. Once qualified by a passing trial weld, welding technicians will not change parameters (temperature, speed, etc.) without performing another trial weld.

\subsubsection{Trial Weld Length}

The trial weld shall be made by joining two pieces of geomembrane at least six inches in width. Trial welds for fusion welds will be 15 feet long and extrusion weld trial seams will be a minimum of four feet long.

\subsubsection{Sample Procedure}

3.4.3.2.1 Visually inspect the seam for squeeze out, footprint, pressure, and general appearance.

3.4.3.2.2 Cut three one-inch wide specimens, one from the middle of the seam and one-foot from each end of the test seam. Specimens shall be obtained using a one-inch die cutter. The specimens shall then be tested in peel using a field tensiometer.

3.4.3.2.3 In order for a trial weld to be considered acceptable, all three specimens must meet the following criteria:

1. Exhibit Film Tearing Bond (FTB).

2. Meet or exceed the manufacturer's minimum peel strength values. If any specimens are in nonconformance, the entire procedure shall be repeated. In the case of double track fusion welded seams, both welds must pass in order to be considered acceptable. 
3.4.3.2.4 If repeat tests utilizing reasonable sets of welding parameters also fail, the seaming apparatus shall not be accepted and shall not be used for seaming until the deficiencies are corrected and a passing test-seam is achieved.

\subsubsection{Trial Weld Documentation}

3.4.3.3.1 The SNLA Delegated Representative will be present during peel testing. The contractor will record date, time, operator, machine number, ambient and operating temperatures, speed setting, peel values, and pass/fail designation.

3.4.3.3.2 All trial weld records shall be maintained and submitted on a trial Weld Form to the SNLA Delegated Representative.

3.4.3.3.3 The SNLA Delegated Representative will give final approval to proceed with welding after observing trial welds.

\subsubsection{General Seaming Procedures}

3.4.4.1 Seaming shall extend to the outside edge of panels to be placed in the anchor trench.

3.4.4.2 While welding a seam, monitor and maintain the proper overlap.

3.4.4.3 Inspect seam area to assure it is clean and free of moisture, dust, dirt and debris of any kind.

3.4.4.4 Welding technicians will periodically check machine operating temperature and speed, and will mark this information on the geomembrane.

3.4.4.5 Align wrinkles at the seam overlap to allow welding through the wrinkle.

3.4.4.6 "Fish mouths" or wrinkles at seam overlaps, which cannot be welded through, shall be cut along the ridge in order to achieve a flat overlap. The cut "fish mouth" or wrinkle shall be heat-tacked flat and extruded or patched with an oval or round patch of the same geomembrane extending a minimum of three inches beyond the cut in all directions.

3.4.4.7 All cross/butt seams between two rows of seamed panels shall be welded during the coolest time of the day to allow for contraction of the geomembrane. 
3.4.4.8 Prior to welding cross/butt seams, the top and bottom overlap of intersecting fusion welded seams will be trimmed six inches. Intersecting extrusion fillet welded seams will be ground to flatten the extrusion bead prior to welding butt seams.

3.4.4.9 All " $\mathrm{T}$ " joints produced as a result of cross/butt seams shall be extrusion fillet welded. The overlap on each "leg" of the "T" joint will be trimmed back six inches. Then grind three inches minimum on each of the three legs of the " $\mathrm{T}$ " and extrusion weld all of the area prepared by grinding.

3.4.4.10 Whenever possible, welding technicians will cut a one-inch peel specimen at the end of every seam. Prior to welding the next seam, the specimen will be tested for peel. The SNLA Delegated Representative may request additional trial welds, based on observations of peel test specimens.

3.4.4.11 In the event non-complying seam test strips are encountered, the welding machine will be taken out of service until a passing trial weld is obtained, and additional peel specimens will be taken to localize the flaw.

3.4.4.12 Results of field seam test strips will be maintained and submitted to the SNLA Delegated Representative.

3.4.4.13 The SNLA Delegated Representative may, after consulting with the Contractor, take destructive samples from any seam, if defects are suspected.

\subsubsection{Seaming Documentation}

3.4.5.1 All seaming operations will be documented by the Contractor and submitted to the SNLA Delegated Representative. Welding technicians will mark on the liner with Mean Streak permanent markers at the start of all seams information regarding date, time, Welding Technician ID, machine number, and machine operating temperature and speed. The Contractor will record date, time, seam number, Technician ID, machine ID, set temperature, speed, and weather condition on a Panel Seaming Form and submit it to the SNLA Delegated Representative.

3.4.5.2 Welding technicians will periodically check operating temperature and speed and mark the information along the seam.

3.4.5.3 The SNLA Delegated Representative will make periodic checks on welding operations to verify overlap, cleanliness, etc. 
4. Seam Testing - Geomembranes

\subsection{Concept}

The welded seam created by a fusion welding process is composed of a primary seam and a secondary track that creates an unwelded channel. The presence of an unwelded channel permits fusion seams to be tested by inflating the sealed channel with air to a predetermined pressure and observing the stability of the pressurized channel over time.

The Contractor shall perform non-destructive air-pressure testing in accordance with the Test Method GM- 6 that has been adopted by the Geosynthetic Research Institute.

\subsection{Air Pressure Testing}

\subsubsection{Equipment for Air Testing}

4.2.1.1 An air pump (manual or motor driven) capable of generating and sustaining a pressure between 20 to $60 \mathrm{psi}$.

4.2.1.2 A rubber hose with fittings and connections.

4.2.1.3 A sharp hollow needle, or other approved pressure feed device with a pressure gauge capable of reading and sustaining a pressure between 0 and $60 \mathrm{psi}$.

\subsubsection{Procedure for Air Testing}

4.2.2.1 Seal both ends of the seam to be tested.

4.2.2.2 Insert needle or other approved pressure feed device into the sealed channel created by the fusion weld.

4.2.2.3 Inflate the test channel to a pressure of approximately $30 \mathrm{psi}$, and maintain the pressure within the range listed in the Initial Pressure Schedule. Close valve, observe and record the initial pressure.

\section{INITIAL PRESSURE SCHEDULE *}

\begin{tabular}{ccc} 
MATERIAL & MINIMUM & MAXIMUM \\
\hline (mils) & (psi) & (psi) \\
40 & 24 & 30
\end{tabular}


*Initial pressure settings are recorded after an optional two minute stabilization period. The purpose of this "relaxing period" is to permit the air temperature and pressure to stabilize. The initial pressure reading may be recorded once stabilization has taken place.

4.2.2.4 Observe and record the air pressure five minutes after the initial pressure setting is recorded. If loss of pressure exceeds the maximum permissible per the manufacturer's recommendation or if the pressure does not stabilize, locate the suspect area and repair in accordance with Section 4.2.3.

4.2.2.5 At the conclusion of all pressure tests, the end of the air-channel opposite the pressure gauge is cut. A decrease in gauge pressure must be observed or the air channel will be considered "blocked" and the test will have to be repeated from the point of blockage. If the point of blockage cannot be found, cut the air channel in the middle of the seam and treat each half as a separate test.

4.2.2.6 Remove the pressure feed needle and seal the resulting hole by extrusion welding.

4.2.3 In the event of a Non-Complying Air Pressure Test, the following procedure shall be followed:

4.2.3.1 Check seam end seals and retest seams.

4.2.3.2 If a seam will not maintain the specified pressure, the seam should be visually inspected to localize the flaw. If this method is unsuccessful, cut one-inch samples from each end of the seam.

4.2.3.3 Perform destructive peel tests on the samples using the field tensiometer.

4.2.3.4 If all samples pass destructive testing remove the overlap left by the wedge welder and vacuum test the entire length of seam in accordance with Paragraph 4.3.

4.2.3.4.1 If a leak is located by the vacuum test, repair by extrusion fillet welding. Test the repair by vacuum testing. 
4.2.3.4.2 If no leak is discovered by vacuum testing, the seam will be considered to have passed non-destructive testing.

4.2.3.5 If one or more peel specimens are in non-compliance, additional samples will be taken in accordance with Paragraph 4.4.3.

4.2.3.5.1 When two passing samples are located, the length of seam bounded by the two passing test locations will be considered non-complying. The overlap left by the wedge welder will be heat tacked in place along the entire length of seam and the non-complying portion of seam will be extrusion fillet welded.

4.2.3.5.2 Test the entire length of the repaired seam by vacuum testing in accordance with Paragraph 4.3.

\subsubsection{General Air Testing Procedures}

4.2.4.1 The opposite end of the air channel will in all cases be pierced to assure that no blockages of the air channel have occurred.

4.2.4.2 Whenever possible, seams should be air-tested prior to completing butt seams to avoid having to cut into liner. All cuts through the liner as a result of testing will be repaired by extrusion welding.

4.2.4.3 All needle holes in air channels remaining after testing will be circled by testing crew and will be repaired with an extrusion bead.

\subsubsection{Air Pressure Testing Documentation}

All information regarding air-pressure testing, (date, initial time and pressure, final time and pressure, pass/fail designation, and Technicians initials) will be written at both ends of the seam, or portion of seam tested. All of the above information will also be logged by the Contractor on a NonDestructive Testing Form and submitted to the SNLA Designated Representative.

\subsection{Vacuum Testing}

This test is used on extrusion welds, or when the geometry of a fusion weld takes air pressure testing impossible or impractical, or when attempting to locate the precise location of a defect believed to exist after air pressure testing.

\subsubsection{Equipment for Vacuum Testing}


4.3.1.1 Vacuum box assembly consisting of a rigid housing with a soft neoprene gasket attached to the bottom, a transparent viewing window, port hole or valve assembly, and a vacuum gauge.

4.3.1.2 Vacuum pump assembly equipped with a pressure controller and pipe connection.

4.3.1.3 A rubber pressure/vacuum hose with fittings and connections.

4.3.1.4 A bucket and means to apply a soapy solution.

4.3.1.5 A soapy solution.

4.3.2 Procedure for Vacuum Testing

4.3.2.1 Trim excess overlap from the seam, if any.

4.3.2.2 Turn on the vacuum pump to reduce the vacuum box to approximately 10 inches of mercury, i.e., 5 psi gauge.

4.3.2.3 Apply a generous amount of a strong solution of liquid detergent and water to the area to be tested.

4.3.2.4 Place the vacuum box over the area to be tested and apply sufficient downward pressure to "seat" the seal strip against the liner.

4.3.2.5 Close the bleed valve and open the vacuum valve.

4.3.2.6 Apply a minimum of 5 psi vacuum to the area as indicated by the gauge on the vacuum box.

4.3.2.7 Ensure that a leak tight seal is created.

4.3.2.8 For a period of approximately 10 to 15 seconds, examine the geomembrane through the viewing window for the presence of soap bubbles.

4.3.2.9 If no bubbles appear after 15 seconds, close the vacuum valve and open the bleed valve, move the box over the next adjoining area with a minimum three inch overlap, and repeat the process.

\subsubsection{Procedure for Non-complying Test}

4.3.3.1 Mark all areas where soap bubbles appear and repair the marked areas in accordance with Paragraph 5.3. 


\subsubsection{Retest repaired areas.}

\subsubsection{General Vacuum Testing Procedures}

4.3.4.1 Vacuum box testing will be performed by qualified construction personnel with frequent supervision by the SNLA Designated Representative.

4.3.4.2 Overlap must be trimmed prior to vacuum boxing all seams.

4.3.4.3 Special attention shall be exercised when vacuum testing " $T$ " seams or patch intersections with seams.

\subsubsection{Vacuum Testing Documentation}

4.3.5.1 Vacuum testing crew will use permanent markers to write on liner indicating tester's initials, date, and pass/fail designation on all areas tested.

4.3.5.2 Records of vacuum testing will be maintained by the Contractor on a Non-Destructive Testing Form or a Repair Report Form and submitted to the SNLA Designated Representative.

\subsection{Destructive Testing}

\subsubsection{Concept}

The purpose of destructive testing is to determine and evaluate seam strength. These tests require direct sampling and thus subsequent patching. Therefore, destructive testing will be held to a minimum to reduce the amount of repairs to the geomembrane.

\subsubsection{Procedure for Destructive Testing}

4.4.2.1 Destructive test samples shall be marked and cut out randomly at a minimum average frequency of one test location every 500 feet of seam length.

4.4.2.2 Location of destructive samples will be selected by the SNLA Designated Representative, with samples cut by the Contractor.

4.4.2.3 Destructive samples should be taken and tested as soon as possible after the seams are welded (the same day), in order to receive test results in a timely manner. 
4.4.2.4 The SNLA Designated Representative will observe all destructive testing. The Contractor will record the date, time, seam number, location, and test results on a Destructive Testing Form and submit to the SNLA Designated Representative.

4.4.2.5 All destructive test locations with pass/fail designation will be marked on liner with permanent Mean Streak markers.

\subsubsection{Sample Size}

4.4.2.6.1 The sample should be twelve inches wide with a seam sixteen inches long centered lengthwise in the sample. The sample may be increased in size to accommodate independent laboratory testing by the Owner, at the Owner's request, or by specific project specifications.

4.4.2.6.2 A one-inch specimen shall be cut from each end of the test seam for field testing.

4.4.2.6.3 The two one-inch wide specimens shall be tested on a field tensiometer for peel strength. If either field specimen does not pass, it will be assumed the sample would also not pass laboratory destructive testing.

The procedure outlined in Paragraph 4.4.3 shall be followed to locate passing samples to send to the laboratory.

\subsubsection{Procedure for Non-Complying Destructive Tests}

4.4.3.1 Cut additional field samples for peel testing. In the case of a field production seam, the samples must lie approximately tenfeet in each direction from the location of the initial noncomplying sample. Perform a field test for peel strength. If these field samples pass, then laboratory samples can be cut and forwarded to the laboratory for full testing.

4.4.3.1.1 If the laboratory samples pass, then reconstruct the seam between the two passing sample locations according to procedures detailed in Section 5.3. 
4.4.3.1.2 If either of the samples are still in non-compliance, then additional samples are taken in accordance with the above procedure until two passing samples are found to establish the zone in which the seam should be reconstructed.

4.4.3.2 All passing seams must be bounded by two locations from which samples passing laboratory destructive tests have been taken.

4.4.3.3 In cases of reconstructed seams exceeding 150 feet, a sample must be taken and pass destructive testing from within the zone in which the seam has been reconstructed.

4.4.3.4 All destructive seam samples shall be numbered and recorded by the Contractor on a Destructive Test Form and submitted to the SNLA Designated Representative.

\subsection{Laboratory Testing of Destructive Seam Samples}

\subsubsection{Destructive Seam Testing Laboratory}

Seam destructive samples will be sent to a testing laboratory to be approved by the SNLA Designated Representative.

\subsubsection{Acceptance Criteria}

Destructive samples will be tested for "Shear Strength" and "Peel Adhesion" (ASTM D4437). Five specimens shall be tested for each test method. Four out of the five specimens must pass for each round of peel and shear testing. In addition, four of the five individual specimens and the average of the five peel and shear tests must meet or exceed the manufacturer's strength requirements in order for the seam to pass the destructive test.

\subsection{Defects and Repairs}

5.1 The SNLA Designated Representative shall conduct a detailed walk through and visually check all seams and non-seam areas of the geomembrane for defects, holes, blisters, and signs of damage during installation.

5.2 All Contractor personnel shall, at all times, be on the lookout for any damaged areas. Damaged areas shall be marked and repaired.

\subsection{Repair Procedures}


Any portion of the geomembrane or geomembrane seam showing a flaw, or having a destructive or non-destructive test in non-compliance shall be repaired. Several procedures exist for repair and the decision as to the appropriate repair procedure shall be made by the Contractor and approved by the SNLA Designated Representative.

Procedures available for repair include the following:

5.3.1 Patching - used to repair large holes, tears, and destructive sample locations. All patches shall extend at least three inches beyond the edges of the defect and all corners of patches shall be rounded.

5.3.2 Grinding and Welding - used to repair sections of extruded fillet seams.

5.3.3 Spot Welding or Seaming - used to repair small tears, pinholes, or other minor localized flaws.

5.3.4 Capping - used to repair lengths of extrusion or fusion welded seams.

5.3.5 Extruding the exposed overlap along the length of fusion welded seams.

5.3.6 Removal of a suspect seam and replacement with of new material seamed into place.

\subsection{Verification of Repairs}

Every repair shall be non-destructively tested using the methods set out in Paragraph 4.4. Repairs which pass the non-destructive test shall be deemed acceptable. Repairs in excess of 150 feet require a destructive test. Nondestructive testing of repairs shall be logged on a Repair Report Form by the Contractor and submitted to the SNLA Designated Representative. The repair location shall be recorded on the record drawing .

\section{End of Section 02776}




\section{SECTION 02778}

\section{GEOTEXTLES}

\subsection{Material}

All geotextile material to be used will be "011/280" Trevira Spunbound nonwoven polyester needlepunched fabric or approved equal.

\subsection{Handling and Placement}

All geotextiles shall be handled in a manner to ensure they are not damaged. The following special handling requirements shall be adhered to:

2.1 On slopes, the geotextiles shall be secured and then rolled down the slope in such a manner as to continually keep the geotextile sheet in sufficient tension to preclude folds and wrinkles.

2.2 In the presence of wind, all geotextiles shall be weighted with sandbags or the equivalent.

2.3 Geotextiles shall be cut using an approved cutter. If the material is being cut in place, special care must be taken to protect other geosynthetic materials from damage.

2.4 Care shall be taken not to entrap stones or excessive dust that could damage the geotextile, or generate clogging of drains or filters.

\subsection{Seams and Overlaps}

Geotextiles will be seamed per the manufacturer's recommendations. No horizontal seams shall be allowed on side slopes.

3.1 On slopes steeper than ten horizontal to one vertical, it is recommended that geotextiles be continuously sewn along the entire length of the seam. Geotextiles shall be overlapped a minimum of four-inches prior to sewing.

3.2 On bottoms and slopes shallower than ten horizontal to one vertical, geotextiles can be either sewn as indicated above or thermally bonded. If thermally bonded the geotextile shall be overlapped a minimum of eight inches prior to seaming.

\subsection{Repairs}


Any holes or tears in the geotextile shall be repaired as follows:

4.1 On Slopes - A patch made from the same geotextile shall be seamed into place. Should any tear exceed $10 \%$ of the width of the roll, that roll shall be removed from the slope and replaced.

4.2 Horizontal Areas - A patch made from the same geotextile shall be spotseamed in place with a minimum of twelve inches overlap in all directions.

End of Section 02778 


\section{SECTION 02779}

\section{DRAINAGE NET (GEONET)}

\subsection{Manufactured Properties}

1.01 Drainage nets shall be manufactured by extruding two sets of polyethylene strands to form a three dimensional structure to provide planar water flow.

1.02 The drainage net shall contain stabilizers to prevent ultraviolet light degradation.

\subsection{Material Properties}

2.01 The drainage net shall be POLY-NET 3000 or approved equal. The drainage net shall conform to the properties detailed below:

\begin{tabular}{|c|c|c|c|c|}
\hline Property & Test Method & Unit & Qualifier & Value \\
\hline Resin Density & ASTM D1505 & $\mathrm{g} / \mathrm{cm}^{2}$ & minimum & 0.940 \\
\hline $\begin{array}{c}\text { Resin Melt } \\
\text { Index }\end{array}$ & ASTM D 1238 & $\mathrm{g} / 10 \mathrm{~min}$ & maximum & 0.5 \\
\hline $\begin{array}{c}\text { Carbon Black } \\
\text { Content }\end{array}$ & ASTM D1603 & $\%$ & minimum & 2 \\
\hline Thickness & ASTM D751 & inches & minimum & 0.200 \\
\hline $\begin{array}{c}\text { Mass per Unit } \\
\text { Area }\end{array}$ & ASTM D3776 & $\mathrm{lbs} / \mathrm{ft}^{2}$ & minimum & 0.162 \\
\hline $\begin{array}{c}\text { Transmissivity } \\
\text { @ 15,000 psf }\end{array}$ & ASTM & $\mathrm{m}^{2} / \mathrm{sec}$ & minimum & $1 \mathrm{X} 10^{-5}$ \\
\hline $\begin{array}{c}\text { Tensile } \\
\text { Strength }\end{array}$ & ASTM D1682 & $\mathrm{lbs} / \mathrm{in}$ & minimum & 42 \\
\hline $\begin{array}{c}\text { Standard Width } \\
\text { X Length }\end{array}$ & -- & feet & -- & $7.54 \mathrm{X} \mathrm{300}$ \\
\hline
\end{tabular}

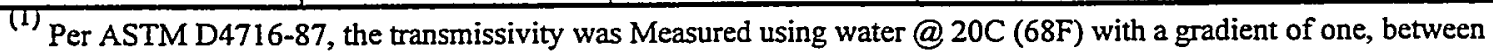
two steel plates, after one hour. Values may vary, based on dimensions of the transmissivity specimen and individual laboratory.

\subsection{Manufacturer's Statement}

3.01 The manufacturer shall submit on request, notarized certifications indicating that the material meets the above specification, signed by an authorized employee of the manufacturer.

3.02 Drainage nets shall be supplied in rolls, marked or tagged with the following information: 

1) Manufacturer's name
2) Product identification
3) Lot number
4) Roll number
5) Roll dimensions

\subsection{Installation}

4.01 Care shall be taken to keep the drainage net clean and free from debris prior to installation. If the drainage net is not clean, it should be washed prior to installation.

4.02 On slopes, drainage nets shall be properly anchored per the manufacturer's recommendations and agreed upon by the SNLA Designated Representative.

4.03 Drainage nets shall not be welded to geomembranes.

4.04 Adjacent rolls shall be overlapped approximately four inches and secured by plastic ties approximately every five-feet along the roll length. Plastic ties shall be white or other bright color for easy inspection. Metallic ties shall not be allowed.

4.05 In corners of the slopes, where overlaps between rolls of nets are staggered, an extra layer of drainage net shall be installed form the top to the bottom of the slope.

4.06 Contractor shall place all cover materials in such a manner to ensure the drainage net and underlying materials are not damaged.

\section{End of Section 02779}




\section{Section 02780}

\section{TEXNET}

\subsection{Related Work.}
A. Section 01300 Descriptive Submittals.
B. Section 02778 Geotextiles.
C. Section 02779 Drainage Nets.

2.0 Material and Product Construction.

1. This product may be substituted for the combination of a geotextile and drainage net. The geotextile is to be placed on top with the drainage net on bottom.

2. The product shall be TexNet by National Seal Company or approved equal.

A. The drainage net shall be as described in Section 02779 of these specifications.

B. The geotextile shall be as described in Section 02778 of these specifications.

C. The texnet shall be manufactured by heat bonding the geotextile to the HDPE drainage net on one side only. No burn through geotextiles shall be permitted. No glue or adhesive shall be permitted.

D. The bond between the geotextile and the HDPE drainage net shall exhibit a minimum average peel strength of 2 pounds per inch, and a typical peel strength of 4 pounds per inch as per ASTM D-413. Peel strength shall be tested approximately every 600 linear feet. These tests are to be performed by the installing contractor in the presence of a SNLA Designated Representative. All results are to be submitted to the SNLA Designated Representative.

The manufacturer shall submit samples of the HDPE drainage net and geotextile to the SNLA Designated Representative with a complete set of specifications for approval. The manufacturer shall also furnish a complete set of instructions for storage, handling, installation, and joining that are to be followed by the installer.

\subsection{Manufacturers Statement}


A. The manufacturer shall submit certifications indicating that the material meets the above specifications and signed by an authorized employee.

B. The TexNet shall be supplied in rolls, marked or tagged with the following:

1. Manufacturer's name,

2. Product identification,

3. Lot number,

4. Roll number,

5. Roll dimensions.

\subsection{Installation}

A. Care shall be taken to keep the texnet clean and free from debris prior to installation. If the texnet is not free of soil and debris before installation, it shall be cleaned by the contractor just prior to installation.

B. On slopes, the texnet shall be properly anchored per manufacturer's recommendations.

C. Adjacent rolls shall be overlapped approximately 2 to 4 inches and secured by plastic ties approximately every five-feet along the roll length. Plastic ties shall be white or another bright color for easy inspection. Metallic ties shall not be allowed. The geotextile will then be either overlapped, sewn, or heat tacked per the manufacturers recommendation. The geocomposite shall not be welded to geomembranes.

D. The installer shall handle all geocomposite rolls in such a manner as to ensure they are not damaged in any way, and the following shall be complied with:

The texnet shall be secured and then rolled down the slope in such a manner as to continually keep the texnet in tension. If necessary, the texnet shall be positioned by hand after being unrolled to minimize wrinkles. The texnet cannot be placed in the horizontal direction (i.e., across the slope).

In the presence of wind, all texnet rolls in place shall be weighted with sandbags or the equivalent. Such sandbags shall be installed during placement and shall remain until replaced with cover material.

E. Contractors shall place all cover materials in such a manner as to ensure the texnet and underlying materials are not damaged.

F. The TexNet is to be installed so that the drainage net is place on the down side on top of the underlying geomembrane. The geotextile will be upward facing with the soil placed on top of it. 


\section{SECTION 02900}

\section{SEEDING}

\section{PART 1 - GENERAL}

\subsection{SCOPE}

This Specification Section covers seeding. All exposed areas not covered by riprap shall be seeded as required to control erosion. The areas to be seeded include all disturbed areas in this contract, as well as, the adjacent area (approximately equal surface area) disturbed in the Phase I contract.

\subsection{MATERIAL STORAGE}

A. Seeds shall be stored in sealed waterproof containers in a cool, dry location and shall be kept out of direct sunlight until ready for use.

B. Fertilizer shall be delivered and stored in waterproof containers that show the chemical analysis and name of manufacturer.

\section{PART 2 - MATERIALS}

\subsection{SEED MTX}

A. Permanent Cover: The following seed mix shall be used for seeding of final grades:

\begin{tabular}{lc} 
Seed Species & $\begin{array}{c}\text { Seeding Rate } \\
\text { Pure Live Seed } \\
\text { (Pounds per Acre) }\end{array}$ \\
\hline Crested Wheatgrass & 3 \\
Indian Ricegrass & 3 \\
Alkali Sacaton & 1 \\
Sand Dropseed & 1 \\
Four-Wing Saltbrush & 1
\end{tabular}

\subsection{ACCEPTANCE OF SEED}


Final acceptance of seed will be made by the SNLA Delegated Representative based on the following:

Seed shall be furnished separately or in mixture in standard sealed containers with the following information provided by the seed vendor on each seed container label: (1) seed name; (2) lot number; (3) net weight; (4) percentages of purity and of germination; (5) seed coverage, in acres, on a pure live seed basis; and (6) percentage of maximum weed seed content clearly marked for each kind of seed. Seeds shall be packaged by the vendor in even acre coverage containers. The Contractor shall furnish the SNLA Delegated Representative duplicate copies of a statement by the vendor, certifying that each lot of seed has been tested by a recognized laboratory for seed testing within six months of date of delivery. This statement shall include: (1) name and address of laboratory: (2) date of test; (3) lot number for each kind of seed; and (4) results of tests as to name, percentages of purity and of germination, and percentage of weed content for each kind of seed furnished, and in case of a mixture, the proportions of each kind of seed.

\subsection{FERTILIZER}

Fertilizer shall be applied at 10 pounds per acre and shall have a 10-10-10 nutrient composition. Fertilizer shall be furnished in new, clean, and sealed containers with the name, weight, and guaranteed analysis of contents clearly marked. A liquid form of fertilizer containing the minimum percentage of available nutrients may be used.

\section{$2.4 \mathrm{MULCH}$}

Hydro-mulch shall be applied over all seeded areas and shall consist of cereal straw. Cereal straw shall be from grain crops that are free from noxious weeds, mold, or other objectionable material. A tackifier shall also be used with the mulch. Final acceptance of mulch will be by the SNLA Designated Representative.

\subsection{WATER}

Water used in the planting or care of vegetation shall be free of oils, acids, alkalis, salts or any substance injurious to plant life.

\section{PART 3 - EXECUTION}

\subsection{PREPARATION}

A. Timing:

1. During Construction (Temporary Cover): No temporary seeding will be required. 
2. Final Grades (Permanent Cover):

a. All disturbed areas not covered by gravel or riprap shall be seeded by using the seed mix specified in Article 2.1 above. The areas to be seeded include all disturbed areas in this contract, as well as, the adjacent area (approximately equal surface area) disturbed in the Phase I contract. Final seeding shall be completed as soon as practical after completion of final grading. Seeding materials shall not be applied during windy weather, when the ground is excessively wet or frozen, or when snow is present.

b. Before applying seed for permanent cover of a given area, the area shall be graded as shown on the Drawings, with surfaces sloping gradually toward drainage courses and no enclosed low spots where water can accumulate. Areas to be seeded that have been damaged by erosion or other causes shall be restored prior to seeding and then cultivated to provide a reasonably firm but friable seedbed. A minimum of six-inches of surface soil shall be in a loose condition at the time of seed application.

\subsection{APPLICATION}

A. Seed for permanent cover shall be applied by a rangeland drill to a depth of 0.5 to 0.75 inch. To the greatest extent possible, seeding shall be oriented along (parallel to) land contours.

\subsection{CARE DURING CONSTRUCTION}

The Contractor shall be responsible for protecting and caring for areas seeded before final acceptance of the work. The Contractor shall repair any damage to seeded areas caused by erosion or construction operations without additional compensation.

\subsection{PLANT ESTABLISHMENT}

Seeding must take place prior to July.

\section{END OF SECTION 02900}




\section{Section 02901}

\section{Sprinkler System Specifications}

\subsection{Related work.}
A. Section 01300 Descriptive Submittals.
B. Section 02200 Earthwork.

2.0 The sprinkler system will be installed as shown on the Contract Drawings. All materials used must be submitted to the SNLA Designated Representative for approval.

The materials outlined on the Contract Drawings are to be used (approved equals are acceptable). The following are specifications for Sprinkler System Components to be used:

Sprinkler Heads

- Rain Jet Water Smart Sprinkler Heads (3" pop-ups)

- Square Pattern - Model \# RS-10 SQ,

- Coverage of 22' X 22'

- Operating Pressure of 15 psi

- Flow rate of $4.5 \mathrm{gpm}$

- Maximum spacing of 22'

Digital Flowmeter: (Sandia furnished, contractor installed)

- Model MT100 by McCrometer

- 3-inch diameter

- Male threads on each end

- Stainless steel construction

- Pressure rating: 150 psi

- Maximum temperature: $160^{\circ} \mathrm{F}$

Electronic Controlled Flowmeter: (Sandia furnished, contractor installed)

- Omega FP-5300

- Polypro Housing

- Titanium Shaft

- For 1/2" - 4" Pipes

- Weight of $.75 \mathrm{lbs}$.

- Length equals 3.50"

- Output Signal: $1 \mathrm{~V}$ p-p/fps

- Output Frequency: 5-6 Hz/fps nominal 
- Flow Rate Range: 1 to $23 \mathrm{fps}$

- Source Impedance: $8 \mathrm{~K}$ ohm

- Maximum Pressure: 180 PSIG maximum at $68^{\circ} \mathrm{F}\left(20^{\circ} \mathrm{C}\right)$

- Maximum Temperature:

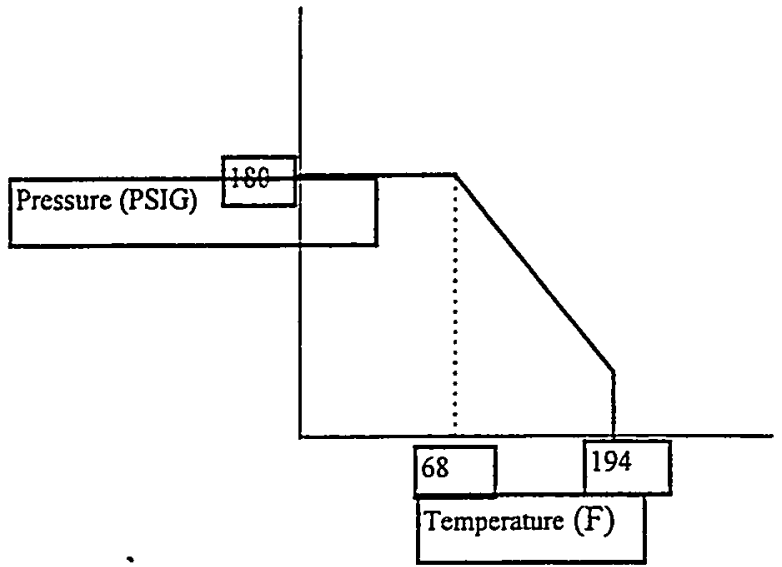

- Pressure Drop: Equal to $8 \mathrm{ft}$. of straight pipe

- Material: Transducer Housing: Glass filled polypropylene; O-Rings: Viton; Shaft: Titanium (PVDF opt.); Rotor: PVDF

- Maximum \% Solids: $1 \%$ of fluid volume, non-abrasive, non-magnetic, $<100$ micron diameter and length

- Standard Cable Length: 25 feet

- Max. Viscosity: 1 centipoise (water); up to $5 \mathrm{cp}$ above 5 fps velocity

- Fittings Required: FP-5320 - 2" sensor installation fitting;

FMK - 31536-1 polypropylene plug

Controller (Sandia furnished, contractor installed)

- Omega DPF75 scaleable totalizer/batch controller, with RS232 output (Sandia furnished, contractor installed)

- Input Power: 110 Vac or $\pm 15 \%$ or 11 to $15 \mathrm{Vdc} ; 220 \mathrm{Vac} \pm 15 \%$ or 11 to 15 Vdc. Max. current $=250 \mathrm{~mA} \mathrm{DC}$ or $32 \mathrm{VA}$ at rated $\mathrm{AC}$ voltage

- Output Power: (AC powered units only)+12 Vdc @50 MA, unregulated +25\%

- Rate Accuracy: $\pm 0.01 \%$ of reading +1 LSD

- K Factor: up to 5 digits

- Temperature: Operating: $+32^{\circ} \mathrm{F}$ to $+130^{\circ} \mathrm{F}$; Storage: $-20^{\circ} \mathrm{F}$ to $200^{\circ} \mathrm{F}$

- Memory: EEPROM stores data for 10 years if power is lost

- Pulse Input: Low =0-1 Vdc; High =4-30Vdc; $10 \mathrm{Kohm}$ impedance; up to 10Khz; $\min .0 .05 \mathrm{~Hz}$ for rate indication.

- Relays: Rated 10 Amps @ 240 Vac or 28 Vdc. SPST, solder jumper selectable for N.O. or N.C., relays latch or autorecycle after 0.1 to 99.9 seconds.

- Panel Cutout: 1.772" H * 3.622"W (DIN standard); 4.06" depth.

- Shipping Weight: $2 \mathrm{lbs}$ 
Amplifier (Sandia furnished, contractor installed)

- Omega FLSC-AMP Amplifier

2-inch Zone Valve and Motor (Sandia furnished, contractor installed)

- Valve, Hardy - part no. RB-EP-200F

- $24 \mathrm{VDC}$

End of Section 02901 


\section{STANDARD SPECIFICATION}

SECTION 03300

\section{CAST-IN-PLACE CONCRETE}

\section{PART 1 - GENERAL}

\subsection{DESCRIPTION OF WORK}

A. Section Includes: Cast-in-place concrete, including formwork, reinforcing, mix design, placement procedures, and finishes.

B. Related Sections: Refer to the following sections for related work:

1. Section 01300 Descriptive Submittals

2. Section 02200 Earthwork

3. Section 05120 Structural Steel

\subsection{REFERENCES}

A. American Society for Testing and Materials (ASTM)

A82 Specification for Steel Wire, Plain, for Concrete Reinforcement

A185 Specification for Steel Welded Wire Fabric, Plain for Concrete Reinforcement

A615 Specification for Deformed and Plain Billet - Steel Bars for Concrete Reinforcement

C33 Specification for Concrete Aggregates

C94 Specification for Ready-Mixed Concrete

C150 Specification for Portland Cement

C260 Specification for Air-Entraining Admixtures for Concrete

C309 Specification for Liquid Membrane-Forming Compounds for Curing Concrete

C494 Specification for Chemical Admixtures for Concrete

C618 Specification for Fly Ash and Raw or Calcined Natural Pozzolan for Use as a Mineral Admixture in Portland Cement Concrete 
C1107 Specification for Packaged Dry, Hydraulic-Cement Grout (nonshrink) -

C1116 Specification for Fiber-Reinforced Concrete and Shotcrete

D994 Specification for Preformed Expansion Joint Filler for Concrete

D1751 Specification for Preformed Expansion Joint Filler for Concrete Paving and Structural Construction

B. Corps of Engineers (CE)

CRD C621 Specification for Non shrink Grout

\subsection{SUBMITTALS}

A. General: Submit the following items in accordance with the Conditions of Contract and Division 1, Section "Descriptive Submittals".

B. Product Data: Submit product data for the following materials and items.

1. Reinforcement

2. Forming Accessories

3. Admixtures

4. Patching Compounds

5. Hardener

6. Joint Systems

7. Curing Compounds

8. Sealants

C. Shop Drawings: Submit shop drawings for fabrication, bending and placement of concrete reinforcement. Show bar schedules, stirrup spacing, diagrams of bent bars and arrangement of reinforcement including bar overlap. Include any special reinforcement required for openings through concrete structures.

D. Laboratory Test Reports: Submit concrete materials test reports and mix design reports certifying that each material or item complies with or exceeds the specified requirements.

\subsection{QUALITY ASSURANCE}

A. Codes and Standards: Comply with provisions of the latest edition or revision of the following, except as otherwise indicated:

1. ACI 301 "Specifications for Structural Concrete for Buildings"

2. ACI 302 "Guide for Concrete Floor and Slab Construction"

3. ACI 304 "Guide for Measuring, Mixing, Transporting and Placing Concrete" 

4. ACI 305 "Hot Weather Concreting"
5. ACI 306 "Cold Weather Concreting"
6. ACI 308 "Standard Practice for Curing Concrete"
7. ACI 309 "Standard Practice for Consolidation of Concrete"
8. ACl 315 "Details and Detailing of Concrete Reinforcement"
9. ACI 318 "Building Code Requirements for Reinforced Concrete"
10. ACI 347 "Recommended Practice for Concrete Formwork"
11. CRSI"Manual of Standard Practice"
12. SP-66 "ACI Detailing Manual"

B. Materials and installed work may require testing and retesting, as directed by the Sandia Delegated Representative, at anytime during progress of work. Retesting of rejected materials and installed work, shall be done at Contractor's expense.

\section{PART 2 - PRODUCTS}

\subsection{FORM MATERIALS}

A. Forms shall be of metal, wood or fiberglass with sufficient thickness to withstand pressure of newly-placed concrete without bow or deflection. Forms for exposed surfaces shall be free of holes, splits, surface voids, and other defects which can affect finished concrete surface.

B. Cvlindrical Columns and Supports: Form round-section members with paper or fiber tubes, constructed of laminated plies using water-resistant adhesive with wax-impregnated exterior for weather and moisture protection. Provide units with sufficient wall thickness to resist loads imposed by wet concrete without deformation.

C. Form Coatings: Provide commercial formulation form-coating compounds that will not bond with, stain, nor adversely affect concrete surfaces, and will not impair subsequent treatments of concrete surfaces.

D. Form Ties: Use factory-fabricated, adjustable-length, removable or snapoff metal form ties, designed to prevent form deflection, and to prevent spalling concrete surfaces upon removal.

1. Unless otherwise indicated, provide ties so portion remaining within concrete after removal is at least $1 / 2 "(12.7 \mathrm{~mm})$ inside concrete for steel ties and $1 / 4^{\prime \prime}(6.35 \mathrm{~mm})$ for wire ties. 
2. Unless otherwise shown, provide form ties which will not leave holes larger than l" (25.4 mm) diameter in concrete surface.

\subsection{REINFORCING MATERIALS}

A. Reinforcing Bars: ASTM A615, deformed.

1. Provide Grade 40 bars No. 3 and 4 for stirrups and ties.

2. Provide Grade 60 bars No. 3 to 18 , except as otherwise noted.

B. Welded steel wire fabric: ASTM A185.

Furnish in flat sheets, not rolls, unless rolls are acceptable to the Sandia Delegated Representative.

C. Cold-drawn steel wire: ASTM A82.

D. Supports for Reinforcement: Provide supports for reinforcement including bolsters, chairs, spacers and other devices for spacing, supporting and fastening reinforcing bars and welded wire fabric in place. Use wire bar type supports complying with CRSI recommendations, unless otherwise indicated. Do not use wood, brick, stone, broken block or pieces of concrete, etc.

E. Fibrous Reinforcement: ASTM C1116.

\subsection{CONCRETEMATERIALS}

A. Portland Cement: ASTM C150 Types I-II and III unless otherwise specified. All types of Portland Cement for paving and sidewalks shall be "low-alkali cement."

B. Aggregates: ASTM C33

C. Water: Potable

D. Fly Ash: ASTM C618, Class F.

E. Admixtures: All admixtures shall be specified in the mix design.

1. Air-Entraining Admixture: ASTM C260

2. Water-Reducing Admixture: ASTM C494, Type A.

3. High Range Water-Reducing Admixture (Super Plasticizer): ASTM C494, Type F or Type G.

4. Water-Reducing, Retarding Admixture: ASTM C494, Type D. 
5. Chloride-containing admixtures are not permitted.

\subsection{RELATED MATERIALS}

A. Expansion Joint Materials: Premolded strips of a bituminous mastic composition conforming to ASTM D994.

B. Non-Shrink Grout: ASTM C1107 (formerly referenced as CE CRD C621), factory pre-mixed grout, non-metallic.

C. Liquid Membrane-Forming Curing Compound: ASTM C309, Type I or I$\mathrm{D}$, Class A.

D. Chemical Hardener: Hardener shall be a colorless, aqueous solution of zinc or magnesium fluosilicate. Approved proprietary hardeners shall be delivered ready for use in the manufacturer's original containers.

E. Bonding Compound: Polyvinyl acetate, rewettable type.

\subsection{CONCRETE MIX DESIGN}

A. General

1. Unless otherwise specified, the minimum strength of concrete shall be 3000 psi $(210.9 \mathrm{kgs}-\mathrm{sq} \mathrm{cm})$ at 28 days.

2. Concrete mixtures shall produce the necessary workability in fresh concrete and the desired strength.

3. Unless otherwise approved or specified, all concrete shall be "ReadyMixed" in accordance with ASTM C94.

4. The water-cement ratio selected shall be that required to produce a 28 day strength corresponding to an over designed mix which is supported by sufficient experience data to assure that test results will fall within the limits established in this specification. Unless otherwise specified, the following proportions apply:

$\begin{array}{cccc}\begin{array}{c}\text { Strength } \\ \text { psi }\end{array} & \begin{array}{c}\text { Min. Cement } \\ \text { Bag/C.Y. }\end{array} & \begin{array}{c}\text { Max. W/C } \\ \text { Ratio }\end{array} & \begin{array}{c}\text { Max. Water } \\ \text { Gal./Bag Cement }\end{array}\end{array}$

3000 ( 210.9 kgs-sq cm) $\quad 5.0$

$4000(281.2 \mathrm{kgs}-\mathrm{sq} \mathrm{cm}) \quad 6.0$

0.60

0.50

6.8

5.6

5. Allowable Slump:

Massive section: $101.6 \mathrm{~mm}$ )

Slabs, beams, walls and columns $\mathrm{mm}$ )

Concrete containing super plasticizer $203.2 \mathrm{~mm}$ ) 
B. Aggregate: Aggregate Grading shall be as follows for the different strengths of concrete.

1. Coarse Aggregate

Shall be as per ASTM C33, Table 2, Grading Requirements for Coarse Aggregates.

\section{Fine Aggregate}

Shall be as per ASTM C33, Sieve Analysis, Fine Aggregate.

C. Admixtures

1. Use water-reducing admixture or high range water-reducing admixture (super plasticizer) in all concrete.

2. Use air-entraining admixture in exterior exposed concrete unless otherwise indicated. Use an air content of 3.5 to 6.5 percent.

3. When an air-entraining admixture is used solely for increasing the workability of the mix, use an air content of 3 to 5 percent.

4. Fly Ash - Unless otherwise specified, a maximum of 9 pounds $(4.08 \mathrm{~kg})$ of fly ash per bag of cement will be permitted.

D. High early strength concrete shall have a compressive strength at 7 days equal to that specified for ordinary concrete at 28 days.

\section{PART 3 - EXECUTION}

\subsection{FORMS}

A. Design. erect support, brace and maintain formwork to support vertical and lateral loads that might be applied until such loads can be supported by concrete structure. Construct formwork so concrete members and structures are of correct size, shape, alignment, elevation and position. Provide for openings, keyways, chamfers, inserts and other features required in work. Maintain formwork construction tolerances, unless otherwise indicated:

1. Variation from Plumb:

a. In the lines and surfaces of columns, piers, walls, and in arises:

In any $10 \mathrm{ft}(3.05 \mathrm{~m})$ of length $1 / 4$ in. $(6.4 \mathrm{~mm})$

Maximum for the entire length 1 in. $(25.4 \mathrm{~mm})$

b. For exposed comer columns, control-joint grooves, and other conspicuous lines:

In any $20 \mathrm{ft}(6.10 \mathrm{~m})$ length $1 / 4$ in. $(6.4 \mathrm{~mm})$ 
2. Variation from the level or from the grades specified in the contract drawings:

a. In slab soffits, ceilings, beam soffits and in arises, measured before removal of supporting shores:

In any $10 \mathrm{ft}(3.05 \mathrm{~m})$ of length $1 / 4$ in. $(6.4 \mathrm{~mm})$ In any bay or in any $20 \mathrm{ft}(6.10 \mathrm{~m})$ length $3 / 8$ in. $(9.7 \mathrm{~mm})$ Maximum for the entire length $3 / 4$ in. $(19 \mathrm{~mm})$

b. In exposed lintels, sills, parapets, horizontal grooves, and other conspicuous lines:

In any bay or in $20 \mathrm{ft}(6.10 \mathrm{~m})$ length $1 / 4$ in. $(6.4 \mathrm{~mm})$ Maximum for the entire length

3. Variation of the linear building lines from established position in plan and related position of columns, walls, and partitions:

In any bay $1 / 2$ in. $(12.7 \mathrm{~mm})$ In any $20 \mathrm{ft}(6.10 \mathrm{~m})$ of length $1 / 2$ in. $(12.7 \mathrm{~mm})$ Maximum for the entire length 1 in. $(25.4 \mathrm{~mm})$

4. Variation in the sizes and location of sleeves, floor openings, and wall openings $1 / 4$ in. $(6.4 \mathrm{~mm})$

5. Variation in cross-sectional dimensions of columns and beams and in the thickness of slabs and walls:

Minus $1 / 4$ in. $(6.4 \mathrm{~mm})$

Plus.. $1 / 2$ in. $(12.7 \mathrm{~mm})$

6. Footings:

a.. Variations in dimensions in plan:

Minus $1 / 2$ in. $(12.7 \mathrm{~mm})$ Plus .2 in. $(50.8 \mathrm{~mm})$

b. Misplacement or eccentricity:

2 percent of the footing width in the direction of misplacement but not more than 2 in. $(50.8 \mathrm{~mm})$

c. Thickness:

Decrease in specified thickness......................5\%

Increase in specified thickness No limit 
7. Variation in Steps:

a. In a flight of stairs:

Rise $+1 / 8$ in. $(+3.2 \mathrm{~mm})$

Tread $+1 / 4$ in. $(+6.4 \mathrm{~mm})$

b. In consecutive steps:

Rise.

Tread $+1 / 16$ in. $(+1.5 \mathrm{~mm})$ $+1 / 8$ in. $(+3.2 \mathrm{~mm})$

* Tolerances apply to concrete dimensions only.

B. Design and fabricate formwork to be readily removable without impact, shock or damage to cast-in-place concrete surfaces and adjacent materials.

C. Chamfer exposed corners and edges as indicated in the drawings to produce uniform smooth lines and tight edge joints.

D. All curb and combined curb and gutter shall be divided into blocks or stones in lengths not to exceed 6 feet $(1.83 \mathrm{~m})$ long using metal templates not less than $1 / 16$ inch $(1.5 \mathrm{~mm})$ thick cut to the same cross section as the curb or curb and gutter being constructed. Templates shall be securely attached to forms to prevent movement during concrete placement.

E. Provisions for Other Trades: Provide openings in concrete formwork to accommodate work of other trades. Determine size and location of openings, recesses and chases from trades providing such items. Accurately place and securely support items built into forms.

A. Comply with CRSI's recommended practice for "Placing Reinforcing Bars", for details and methods of reinforcement placement and supports, and as herein specified. Field bending of grade 60 bars is not permitted.

B. Clean reinforcement of loose rust and mill scale, earth, ice, oil, concrete splatter from previous pours, and other materials which reduce or destroy bond with concrete.

C. Accurately position. support and secure reinforcement against displacement by formwork, construction, or concrete placement operations. Locate and support reinforcing by metal chairs, runners, bolsters, spacers, and hangers, as required.

D. Install welded wire fabric of same gage in as long of lengths as practicable. Lap adjoining pieces at least one full mesh and lace splices with wire. Offset end laps of adjacent widths to prevent continuous laps in either direction.

E. Provide minimum cover for reinforcement of cast-in-place concrete, unless otherwise indicated. 
Concrete cast against and permanently exposed to earth..........3 in. (76.2 mm) Concrete exposed to earth or weather

$\# 6$ and larger

$\# 5$ and smaller $11 / 2$ in. $(38.1 \mathrm{~mm})$

Concrete not exposed to weather or in contact with earth

Slabs, walls, joists $3 / 4$ in. $(19 \mathrm{~mm})$

Beams, columns $11 / 2$ in. $(50.8 \mathrm{~mm})$

\subsection{JOINTS}

A. Construction Joints

1. Locate construction joints on slab floor, which are not shown on drawings, and notify Sandia Delegated Representative for approval.

2. Provide keyways at least $1-1 / 2 "(38.1 \mathrm{~mm})$ deep in construction joints in walls and between walls and footings; accepted preformed keyways designed for this purpose may be used for slabs.

3. Place construction joints perpendicular to the main reinforcement. Continue reinforcement across construction joints.

B. Isolation Joints: Construct isolation joints in slabs-on-ground at points of contact between slabs on ground and foundations as shown on drawings. Unless otherwise indicated, install 90\# felt paper between slab and vertical surface.

C. Contraction (Control) Joints

1. Contraction Joints in Slabs-on-Ground: Construct contraction joints in slabs-on-ground to form panels of patterns as shown. Contraction joints in floor slabs shall be formed with preformed metal bulkheads.

2. Walks: Contraction joints shall not exceed intervals of 6 feet $(1.828 \mathrm{~m})$. Joints shall be made at regular intervals along the line of the work. On straight work, the joints shall be parallel with and at right angles to the line of the work; at curves the joints shall, in general, be along lines concentric with and radial to the proportion of the work in which they are placed. The markings shall be made with jointer tools that will round the edges of the scoring lines to a radius of $1 / 8$ inch $(3.2 \mathrm{~mm})$, with a depth of not less than $11 / 4$ inch $(31.75 \mathrm{~mm})$. The finished joint opening, exclusive of radii, shall not be wider than $1 / 8$ inch $(3.2 \mathrm{~mm})$.

D. Expansion Joints

1. Slabs-on-Ground: Expansion joint material shall be placed around utility access openings within the slab, including clean outs and utility valves, and between new concrete slab and adjacent masonry. Joint material shall be $1 / 2$ inch $(12.7 \mathrm{~mm})$ thick, premolded, asphalt impregnated complying with ASTM D1751 and extend the full depth of the concrete. 
2. Curbs and Gutters: Construct $1 / 2$ inch $(12.7 \mathrm{~mm})$ joints in curbs and gutters at the end of all returns except where cross gutters are being constructed. They shall be at the ends of the cross gutter transitions and also along the line of work at regular intervals, not to exceed 36 feet $(10.97 \mathrm{~m})$. Joints in gutter shall be continuous with those in adjacent curb.

3. Walks: Place joints in walks at the point of tangency (PT) and point of curvature (PC) of all walk returns, between walk and a building or structure, in walk returns, between the walk and the back of the curb returns and around all utility poles. At the PT and PC and around utility poles, the joint filler strips shall extend the full depth of the concrete being placed. Joint filler strips between walk and curb shall be the full depth of the walk with the top of the filler strip set flush with the top of the curb. Transverse sidewalk joints shall not exceed 30 feet $(9.14 \mathrm{~m})$ spacing. Joint material shall be $1 / 2$ inch $(12.7 \mathrm{~mm})$ thick.

\subsection{PREPARATIONS FOR PLACING CONCRETE}

A. Water shall be removed from excavations. Wood chips, shavings, and hardened concrete shall be removed from the forms before any concrete is placed. All equipment shall be cleaned. Forms shall be wetted, except in freezing weather, or oiled.

B. The earth shall be uniformly moist when the concrete is placed. The method of sprinkling shall not be such as to form mud or pools of water. Watering the subgrade immediately prior to placing the concrete is not sufficient to make the soil uniformly moist.

C. Notify other crafts to permit installation of their work. Coordinate the installation of joint materials and moisture barriers with placement of forms and reinforcing steel.

\subsection{PLACING CONCRETE}

\section{A. Field Inspection}

1. Concrete shall not be placed until forms and reinforcing steel have been inspected and approved.

2. Ready-Mix concrete shall be placed within a specified delivery time:

$\begin{array}{ll}\text { Below } 40^{\circ} \mathrm{F}\left(4.4^{\circ} \mathrm{C}\right) & \text { see cold weather placing } \\ 40^{\circ}-85^{\circ} \mathrm{F}\left(4.4-29.4^{\circ} \mathrm{C}\right) & 90 \text { minutes } \\ 86^{\circ}-90^{\circ} \mathrm{F}\left(30^{\circ}-32.2^{\circ} \mathrm{C}\right) & 75 \text { minutes } \\ \text { above } 90^{\circ} \mathrm{F}\left(32.2^{\circ} \mathrm{C}\right) & 60 \text { minutes }\end{array}$

Concrete exceeding delivery time may be rejected by the Sandia Delegated Representative.

3. Adding Water: No water shall be added after the initial introduction of mixing water for the batch except, when on arrival at the job site, the 
slump of the concrete is less than that specified, and the maximum water/cement ratio for the mix has not been exceeded. Such additional water shall be added to bring the slump within limits. The drum shall be turned at least 30 additional revolutions at mixing speed. The Sandia Delegated Representative shall be notified before adding any water. Water shall not be added to the batch at any later time.

The contractor shall insure that the concrete strength meets specifications and that the maximum amount of water as noted in the CONCRETE MIX DESIGN is not exceeded. Comply with ASTM C 94.

B. General: Comply with $\mathrm{ACI} 304$, and as herein specified. Deposit concrete continuously or in layers of such thickness that no concrete will be placed on concrete which has hardened sufficiently to cause the formation of seams or planes of weakness. If a section cannot be placed continuously, provide construction joints as herein specified. Deposit concrete as nearly as practicable to its final location to avoid segregation.

C. Placing Concrete in Forms

1. Deposit concrete in forms in horizontal layers not deeper than 24 " $(609.6$ $\mathrm{mm}$ ) and in a manner to avoid inclined construction joints. Where placement consists of several layers, place each layer while preceding layer is still plastic to avoid cold joints.

2. Consolidate placed concrete by high frequency mechanical vibrating equipment, supplemented, as necessary, by hand- spading, rodding or tamping. Use equipment and procedures for consolidation of concrete in accordance with ACI 309.

3. Do not use vibrators to transport concrete inside forms. Insert and withdraw vibrators vertically at uniformly spaced locations not farther than visible effectiveness of machine. Place vibrators to rapidly penetrate placed layer and at least 6" $(152.4 \mathrm{~mm})$ into preceding layer. Do not insert vibrators into lower layers of concrete that have begun to set. At each insertion limit duration of vibration to time necessary to consolidate concrete and complete embedment of reinforcement and other embedded items without causing segregation of mix.

4. Concrete shall not be allowed to free fall more than 5 feet $(1.52 \mathrm{~m})$ unless confined by a closed chute, except that concrete being placed in walls 10 inches $(254 \mathrm{~mm})$ or less in thickness may free fall a maximum of 8 feet $(2.44 \mathrm{~m})$.

\section{Placing Concrete Slabs}

1. Deposit and consolidate concrete slabs in a continuous operation, within limits of construction joints, until the placing of a panel or section is completed.

2. Bring slab surfaces to correct level as above, and use bull floats or darbies to smooth surface, free of humps or hollows. Do not use tools (i.e., jitterbugs) that force the aggregate away from the surface. 
3. Maintain reinforcing in proper position during concrete placement operations.

E. Cold Weather Placing: Protect concrete work from physical damage or reduced strength which could be caused by frost, freezing actions, or low temperatures; comply with ACI 306 and these specifications.

1. Concrete shall be mixed and placed only when the temperature is at least $40^{\circ} \mathrm{F}\left(22^{\circ} \mathrm{C}\right)$ and rising unless permission to pour is obtained from the Sandia Delegated Representative.

2. Do not use frozen materials or materials containing ice or snow. Do not place concrete on frozen subgrade or on subgrade containing frozen materials.

3. Do not use calcium chloride, salt and other materials containing antifreeze agents or chemical accelerators.

4. When approval is obtained to place concrete at or below an atmospheric temperature of $40^{\circ} \mathrm{F}\left(22^{\circ} \mathrm{C}\right)$, the water or aggregates, or both, shall be heated, and suitable enclosures and heating devices shall be provided. The mixed concrete shall have a temperature of at least $50^{\circ} \mathrm{F}\left(28^{\circ} \mathrm{C}\right)$ and not more than $90^{\circ} \mathrm{F}\left(50^{\circ} \mathrm{C}\right)$ at the time of placing.

The heating equipment or methods shall be capable of heating the water and aggregates uniformly, and these materials shall not be heated to a temperature exceeding $150^{\circ} \mathrm{F}\left(83^{\circ} \mathrm{C}\right)$.

5. After any concrete is placed, the Contractor shall provide suitable measures to maintain a concrete surface temperature of $40^{\circ} \mathrm{F}\left(22^{\circ} \mathrm{C}\right)$ or above for a period of not less than 7 days.

F. Hot Weather Placing: When hot weather conditions exist that would seriously impair quality and strength of concrete, place concrete in compliance with ACI 305 and as herein specified.

1. Cool ingredients before mixing to maintain concrete temperature at time of placement below $90^{\circ} \mathrm{F}\left(50^{\circ} \mathrm{C}\right)$.

2. Cover reinforcing steel with water-soaked burlap when required to ensure that steel temperature will not exceed the ambient air temperature immediately before embedment in concrete.

3. Wet forms thoroughly before placing concrete.

4. Use water-reducing retarding admixture (Type D) when required by high temperatures, low humidity, or other adverse placing conditions.

\subsection{CONCRETE FINISHING}

\section{A. General}


1. Do not use tools (i.e., jitterbugs) that force the aggregate away from the surface.

2. Water shall not be sprayed or sprinkled onto a concrete surface to aid in finishing.

3. The finisher should avoid bringing more water than necessary to the surface and avoid working the surface any more than necessary to obtain the required finish.

\section{B. Slab Finishes}

1. Float Finish: Apply float finish to slabs for driveways, exterior slabs, curbs and gutters and interior floor slabs receiving additional covering.

After screeding, consolidating, and leveling concrete slabs, do not work surface until ready for floating. Begin floating, using float blades or float shoes only, when surface water has disappeared, when concrete has stiffened sufficiently to permit operation of powerdriven floats, or both. Consolidate surface with power-driven floats or by hand-floating if area is small. Check and level surface plane to a tolerance of Class B. Class B tolerances shall be true planes within $1 / 4$ inch $(6.4 \mathrm{~mm})$ in $10 \mathrm{ft}$. $(3.05 \mathrm{~m})$, as determined by a $10-\mathrm{ft}$. $(3.05$ $\mathrm{m})$ straightedge placed anywhere on the slab in any direction.

2. Trowel Finish: Apply trowel finish to interior exposed floors.

After floating, begin first trowel finish operation. Begin final troweling when surface produces a ringing sound as trowel is moved over surface. Consolidate concrete surface by final hand-troweling operation, free of trowel marks, uniform in texture and appearance and with surface leveled to a Class B tolerance.

3. Light Broom: For sidewalks apply trowel finish as specified above. Immediately follow with slightly scarifying surface by fine brooming perpendicular to main traffic route.

C. Finish of Formed Surfaces

1. Grout-Cleaned Finish: The entire surface of all exposed exterior concrete shall be given a rubbed grout clean-down finish using a mix of one part white Portland cement and 1-1/2 parts of fine sand mixed with sufficient water to form a grout having the consistency of thick paint. Apply to damp surface and rub down in such a manner as to obtain a smooth, filled surface uniform in color and free from defects and blemishes.

2. Smooth Rubbed Finish: Interior exposed surfaces (where scheduled to be painted) shall be rubbed with carborundum stones and water to a smooth, even surface for painting.

\subsection{CONCRETE CURING}


A. General: Immediately after placing or finishing, and as soon as the operation will not mar the finish, concrete surfaces not covered by forms shall be protected against moisture loss. Curing shall be accomplished by one of the following methods or a combination thereof, as approved, and such protection shall be maintained for a period of at least 7 days. Where formed surfaces are cured in the forms, the forms shall be kept continually wet. If the forms are removed before the end of the curing period, curing shall be continued as on unformed surfaces, using curing materials specified herein. Surfaces shall be kept free of foot and vehicular traffic during the curing period.

B. Curing Methods

1. Polyethylene Coated Burlap Mats: Surfaces shall be covered with the specified mat lapped 12 inches $(304.8 \mathrm{~mm})$. The mat shall be weighted to prevent displacement. Tears or holes shall be immediately repaired by patching.

2. Membrane Forming Curing Compound: This compound shall be applied in a two coat continuous operation, using not less than the manufacturer's recommended rate of application. If unknown, it shall be applied at 1 gallon (3.79 liters) per 200 square feet (18.58 square meters) for each coat. Surfaces damaged by construction operations during curing shall be resprayed at the same rate.

3. Water Curing: Water curing shall be used on surfaces that are to receive additional concrete or concrete fill, dustproofing and hardening treatments, stucco, plaster, or painting.

\subsection{REMOVAL OF FORMS}

The forms for any portion of the structure shall not be removed until the concrete is strong enough to not be damaged when the forms are removed.

A. If field operations are not controlled by cylinder tests, the following periods, exclusive of days when the temperature is below $40^{\circ} \mathrm{F}\left(4.4^{\circ} \mathrm{C}\right)$, may be used as a guide for removal of forms and supports:

Centering under beams

Elevated floor slabs

Walls

Columns

Sides of beams and all other parts
14 days

$7-14$ days*

12-24 hours*

1-7 days*

12-24 hours*

*The longer time dictates unless the Sandia Delegated Representative approves the lesser time.

B. If field operations are controlled by beam or cylinder tests, forms may be removed from centering under beams and floor slabs when a 2500 psi 
(175.77 kgs-sq cm) compressive strength is attained, and it is approved by the Sandia Delegated Representative.

C. Superimposed loads shall not be placed on or against load carrying members until a $2500 \mathrm{psi}(175.77 \mathrm{kgs}-\mathrm{sq} \mathrm{cm}$ ) compressive strength has been attained and it is approved by the Sandia Delegated Representative.

\subsection{REUSE OF FORMS}

Clean and repair surfaces of forms to be reused in work. The shape, strength, rigidity, water-tightness, and surface smoothness of reused forms shall be maintained at all times. Any warped or bulged lumber shall be re-sized before being used. Unsatisfactory forms shall not be used.

\subsection{CONCRETE SURFACE REPAIRS}

A. Patching Defective Areas: Immediately after removing forms, cut back form ties and remove all fins and loose material. Honeycombs and voids shall be cut to solid concrete, thoroughly wetted, and filled with cement mortar composed of one part cement to three parts sand.

B. Repair of Formed Surfaces: Remove and replace concrete having defective surfaces if defects cannot be repaired to satisfaction of Inspector. Surface defects include color and texture irregularities, cracks, spalls, air bubbles, honeycomb, rock pockets, fins and other projections on surface, and stains and other discolorations that cannot be removed by cleaning. Flush out form tie holes, fill with dry-pack mortar.

C. Repair of Slabs-on-Ground: Test surfaces for smoothness and verify surface plane to tolerances specified for each surface and finish. Correct low areas during or immediately after completion of surface finishing operations by cutting out low areas and replacing with patching compound. Finish repaired areas to blend into adjacent concrete. Proprietary underiayment compounds may be used when accepted per 1.04 of this specification. Correct high areas in slabs by grinding after concrete has cured at least 14 days.

\subsection{CONCRETE TRUCK DISCHARGE}

A. Excess Concrete: Excess concrete in mixer trucks that cannot be immediately used shall be discharged to an area where it will not create an obstruction or hazard during construction. This concrete shall be removed from the site in a timely manner to a site approved by the Sandia Delegated Representative.

B. Wash Water Discharge: Wash water from mixer trucks shall be discharged to the ground surface in such a manner and at such a location that discharge can not escape the construction site or be washed away to arroyos, storm sewers, or sanitary sewers by precipitation or other surface flows. Prior to project completion, wash water residue shall be removed from the site to a 
location approved by the Sandia Delegated Representative and the wash water discharge site shall be cleaned free of debris.

\section{END OF SECTION 03300}




\section{STANDARD SPECIFICATION \\ SECTION 05120 \\ STRUCTURAL STEEL}

\section{PART I - GENERAL}

\subsection{DESCRIPTION OF WORK}

A. This section includes fabrication and erection of structural steel work, as shown on drawings including schedules, notes, and details showing size and location of members, typical connections, and types of steel required.

Structural steel is that work defined in American Institute of Steel Construction (AISC) "Code of Standard Practice for Steel Buildings and Bridges" and as otherwise shown on drawings.

B. Related Sections: Refer to the following sections for related work:

1. Division 3, Section "Cast-In-Place Concrete" for anchor bolt and reinforcing steel installation in concrete.

2. Division 5, Section "Metal Fabrications" for miscellaneous metal fabrications.

3. Division 9, Section "Painting" for priming and painting of steel.

\subsection{REFERENCES}

\section{A. American Society of Testing and Materials (ASTM)}

A6 Specification for General Requirements for Delivery of Rolled Steel Plates, Shapes, Sheet Piling and Bars for Structural Use

A27 Specification for Steel Castings, Carbon, for General Application

A36 Specification for Structural Steel

A53 Specification for Pipe, Steel, Black and Hot-Dipped, Zinc-Coated Welded and Seamless

A108 Specification for Steel Bars, Carbon, Cold-Finished, Standard Quality 
A153 Specification for Zinc Coating (Hot-Dip) on Iron and Steel Hardware

A307 Specification for Carbon Steel Bolts and Studs, 60000 psi Tensile Strength

A325 Specification for High-Strength Bolts for Structural Steel Joints

A500 Specification for Cold-Formed Welded and Seamless Carbon Steel Structural Tubing in Rounds and Shapes

A501 Specification for Hot-Formed Welded and Seamless Carbon Steel Structural Tubing

B695 Specification for Coatings of Zinc Mechanically Deposited on Iron and Steel

C1107 Specification for Packaged Dry, Hydraulic-Cement Grout (Nonshrink)

E94 Guide for Radiographic Testing

E142 Method for Controlling Quality of Radiographic Testing

E164 Practice for Ultrasonic Contact Examination of Weldments

E165 Practice for Liquid Penetrant Inspection Method

E709 Practice for Magnetic Particle Examination

F959 Specification for Compressible-Washer-Type Direct Tension Indicators for Use with Structural Fasteners

B. American Welding Society (AWS)

D1.1 Structural Welding Code - Steel

C. Corps of Engineers (CE)

CRD C621 Specification for Nonshrink Grout

D. Federal Specification (FS)

TT-P-664 Primer Coating, Alkyd, Corrosion-Inhibiting, Lead and Chromate Free, VOC-Compliant

E. National Fire Protection Assoc. (NFPA) 
51B Standard for Fire Prevention in Use of Cutting and Welding Processes

F. Steel Structures Painting Council (SSPC)

SP-1 Solvent Cleaning

SP-2 Hand-Tool Cleaning

SP-3 Power-Tool Cleaning

\subsection{SUBMITTALS}

A. General: Submit the following in accordance with conditions of Contract and Division 1, Section "Descriptive Submittals".

B. Product Data: Submit product data or manufacturer's specifications and installation instructions for the following products. Include laboratory test reports and other data to show compliance with specifications (including specified standards).

1. Structural steel (each type), including certified copies of mill reports covering chemical and physical properties.

2. Anchor bolts.

3. Unfinished threaded fasteners.

4. High-strength bolts (each type), including nuts and washers; include direct tension indicators if used.

5. Structural steel primer paint.

6. Nonmetallic shrinkage-resistant grout.

C. Material Safety Data Sheets (MSDS): Submit MSDS for structural steel (each type), anchor bolts, unfinished threaded fasteners, high-strength bolts (each type) including nuts and washers, structural steel primer paint and nonmetallic shrinkage-resistant grout.

D. Shop drawings: Submit shop drawings, including complete details and schedules for fabrication and assembly of structural steel members, procedures, and diagrams. 
1. Include details of cuts, connections, cambers, holes and other pertinent data. Indicate welds by standard AWS symbols and show size, length, and type of each weld.

2. Provide setting drawings, templates, and directions for installation of anchor bolts and other anchorages to be installed as work of other sections.

E. Welder Certifications: Provide certification that welders to be employed in work have satisfactorily passed qualification tests in accordance with AWS D1.1.

If recertification of welders is required, retesting will be Contractor's responsibility.

F. Test reports: Submit test reports conducted on shop- and field-bolted and welded connections. Include data on type(s) of tests conducted and test results.

\subsection{QUALITY ASSURANCE}

A. Codes and Standards: Comply with provisions of the following, except as otherwise indicated:

1. AISC "Code of Standard Practice for Steel Buildings and Bridges" with paragraph 4.2.1 modified by deletion of the following sentence:

"This approval constitutes the owner's acceptance of all responsibility for the design adequacy of any detail configuration of connections developed by the fabricator as a part of his preparation of these shop drawings."

2. AISC "Specifications for Structural Steel Buildings" including the "Commentary", later referred to as "AISC Specifications".

3. "Specifications for Structural Joints using ASTM A325 or A490 Bolts" approved by the Research Council on Structural Connections.

\section{AWS D1.1}

\section{ASTM A6}

B. Qualifications for Welding Work: Qualify welding procedures and welding operators in accordance with the requirements of AWS D1.1. 
C. All materials used shall not contain asbestos fibers.

\subsection{DELIVERY. STORAGE AND HANDLING}

A. Deliver materials to site at such intervals to ensure uninterrupted progress of work.

B. Deliver anchor bolts and anchorage devices, which are to be embedded in cast-in-place concrete or masonry, in ample time so that work will not be delayed.

C. Store materials to permit easy access for inspection and identification. Keep steel members off ground by using pallets, platforms, or other supports. Protect steel members and packaged materials from corrosion and deterioration. If bolts and nuts become dry or rusty, clean and lubricate before use.

Do not store materials on structure in a manner that might cause distortion or damage to members or supporting structures. Repair or replace damaged materials or structures as directed.

\subsection{PROJECT CONDITIONS}

Field Measurements: Check actual locations of walls and other construction to which steel framing must fit, by accurate field measurements before fabrication; show recorded measurements on final shop drawings.

\section{PART 2 - PRODUCTS}

\subsection{MATERIALS}

A. Metal Surfaces. General: For fabrication of work that will be exposed to view, use only materials that are smooth and free of surface blemishes including pitting, rust and scale seam marks, roller marks, rolled trade names, and roughness. Remove such blemishes by grinding, or by welding and grinding, prior to cleaning, treating, and applying surface finishes.

B. Structural Steel Shapes, Plates. and Bars: ASTM A36 


\section{Cold-Formed Steel Tubing: ASTM A500, Grade B. \\ D. Hot-Formed Steel Tubing: ASTM A501. \\ E. Steel Pipe: ASTM A53, Type E or S, Grade B: or ASTM A501.}

Finish: Black, except where indicated to be galvanized.

F. Steel Castings: ASTM A27, Grade 65-35, medium-strength carbon steel.

G. Headed Stud-Type Shear Connectors: ASTM A108, Grade 1015 or 1020, cold-finished carbon steel with dimensions complying with AISC Specifications.

H. Anchor Bolts: ASTM A307, nonheaded type unless otherwise indicated.

I. Unfinished Threaded Fasteners: ASTM A307, Grade A, regular low-carbon steel bolts and nuts; provide hexagonal heads and nuts for all connections.

J. High-Strength Threaded Fasteners: Heavy hexagon structural bolts, heavy hexagon nuts, and hardened washers, as follows:

1. Quenched and tempered medium-carbon steel bolts, nuts and washers, complying with ASTM A325.

2. Where indicated as galvanized, provide units that are zinc-coated, either mechanically deposited complying with ASTM B 695 , Class 50, or hotdip galvanized complying with ASTM A153.

K. Direct Tension Indicators: ASTM F959, type as required; use at Contractor's option.

L. Electrodes for Welding: Comply with AWS Code.

M. Structural Steel Primer Paint: Red oxide, lead- and cadmium-free, corrosion-inhibiting primer complying with performance requirements of FS TT-P-664.

N. Nonmetallic Shrinkage-Resistant Grout: Premixed, nonmetallic, noncorrosive, nonstaining product containing selected silica sands, Portland cement, shrinkage compensating agents, plasticizing and water-reducing agents, complying with ASTM C1107 (formerly referenced as CE CRD C621). 
Subject to compliance with requirements, products that may be incorporated in the work include, but are not limited to, the following:

100 Non-Shrink Grout (Non-Metallic) - Conspec, Inc.

Crystex - L \& M Construction Chemicals, Inc.

Euco N-S Grout - Euclid Chemical Co.

Kemset - Chem-Masters Corp.

Sonogrout - Sonneborn Building Products Div., Rexnord Chemical Products, Inc.

Supreme Grout - Cormix, Inc.

Sure-Grip High Performance Grout - Dayton Superior

Vibropruf\#11 - Lambert Corp.

\subsection{FABRICATION}

A. Shop Fabrication and Assembly: Fabricate and assemble structural assemblies in shop to greatest extent possible. Fabricate items of structural steel in accordance with AISC Specifications and as indicated on final shop drawings. Provide camber in structural members where indicated.

1. Properly mark and match-mark materials for field assembly. Fabricate for delivery sequence that will expedite erection and minimize field handling of materials.

2. Where finishing is required, complete assembly, including welding of units, before start of finishing operations. Provide finish surfaces of members exposed in final structure free of markings, burrs, and other defects.

B. Connections: Weld or bolt shop connections, as indicated.

1. Bolt field connections, except where welded connections or other connections are indicated.

2. Provide high-strength threaded fasteners, unless otherwise indicated.

C. High-Strength Bolted Connections: Install high-strength threaded fasteners in accordance with AISC "Specifications for Structural Joints using ASTM A325 or A490 Bolts". 
D. Welded Construction: Comply with AWS Code for procedures, appearance and quality of welds, and methods used in correcting welding work.

E. Shear Connectors: Prepare steel surfaces as recommended by manufacturer of shear connectors. Weld shear connectors in field, spaced as shown, to beams and girders in composite construction. Use automatic end welding of headed stud shear connectors in accordance with manufacturer's printed instructions.

F. Steel Wall Framing: Select members that are true and straight for fabrication of steel wall framing. Straighten as required to provide uniform, square, and true members in complete wall framing.

Build up welded door frames attached to structural steel framing. Weld exposed joints continuously and grind smooth. Plug-weld steel bar stops to frames, except where shown removable. Secure removable stops to frames with countersunk, cross-recessed head machine screws, uniformly spaced not more than 10 inches $(25 \mathrm{~cm})$ o.c., unless otherwise indicated.

G. Holes for Other Work: Provide holes required for securing other work to structural steel framing and for passage of other work through steel framing members, as shown on final shop drawings.

1. Provide threaded nuts welded to framing and other specialty items as indicated to receive other work.

2. Cut, drill, or punch holes perpendicular to metal surfaces. Do not flamecut holes or enlarge holes by burning. Drill holes in bearing plates.

\subsection{SHOP PAINTING}

A. General: Shop-paint structural steel, except those members or portions of members to be embedded in concrete or mortar. Paint embedded steel that is partially exposed on exposed portions and initial 2 inches $(51 \mathrm{~mm})$ of embedded areas only.

1. Do not paint surfaces to be welded or high-strength bolted with frictiontype connections.

2. Do not paint surfaces scheduled to receive sprayed-on fireproofing.

3. Apply 2 coats of paint to surfaces that are inaccessible after assembly or erection. Change color of second coat to distinguish it from first. 
B. Surface Preparation: After inspection and before shipping, clean steelwork to be painted. Remove loose rust, loose mill scale, and spatter, slag, or flux deposits. Clean steel in accordance with SSPC as follows:

SP-1 "Solvent Cleaning"

SP-2 "Hand-Tool Cleaning"

SP-3 "Power-Tool Cleaning"

C. Painting: Immediately after surface preparation, apply structural steel primer paint in accordance with manufacturer's instructions and at a rate to provide dry film thickness of not less than 3.0 mils. Use painting methods that result in full coverage of joints, corners, edges, and exposed surfaces.

\subsection{SOURCE QUALITY CONTROL}

Materials and fabrication procedures are subject to inspection and tests in mill, shop, and field, conducted by a qualified inspection agency. Such inspections and tests will not relieve Contractor of responsibility for providing materials and fabrication procedures in compliance with specified requirements. Promptly remove and replace materials or fabricated components that do not comply.

\section{PART 3 - EXECUTION}

\subsection{ERECTION}

A. Temporary Shoring and Bracing: Provide temporary shoring and bracing members with connections of sufficient strength to bear imposed loads. Remove temporary members and connections when permanent members are in place and final connections are made. Provide temporary guy lines to achieve proper alignment of structures as erection proceeds.

B. Anchor Bolts: Furnish anchor bolts and other connectors required for securing structural steel to foundations and other in-place work.

Furnish templates and other devices as necessary for presetting bolts and other anchors to accurate locations.

C. Setting Bases and Bearing Plates: Clean concrete and masonry bearing surfaces of bond-reducing materials and roughen to improve bond to surfaces. Clean bottom surface of base and bearing plates. 
1. Set loose and attached base plates and bearing plates for structural members on wedges or other adjusting devices.

2. Tighten anchor bolts after supported members have been positioned and plumbed. Do not remove wedges or shims, but if protruding, cut off flush with edge of base or bearing plate prior to packing with grout.

3. Pack grout solidly between bearing surfaces and bases or plates to insure that no voids remain. Finish exposed surfaces, protect installed materials, and allow to cure.

4. For proprietary grout materials, comply with manufacturer's instructions.

D. Field Assembly: Set structural frames accurately to lines and elevations indicated and in accordance with AISC Specifications. Align and adjust various members forming part of complete frame or structure before permanently fastening. Clean bearing surfaces and other surfaces that will be in permanent contact before assembly. Perform necessary adjustments to compensate for discrepancies in elevations and alignment.

1. Level and plumb individual members of structures within specified AISC tolerances.

2. Establish required leveling and plumbing measurements on mean operating temperature of structure. Make allowances for difference between temperature at time of erection and mean temperature at which structure will be when completed and in service.

3. Splice members only where indicated and accepted on shop drawings.

4. Comply with AISC Specifications for bearing, adequacy of temporary connections, alignment, and removal of paint on surfaces adjacent to field welds.

5. Do not enlarge unfair holes in members by burning or by using drift pins, except in secondary bracing members. Ream holes that must be enlarged to admit bolts.

E. Gas Cutting: Do not use gas cutting torches in field for correcting fabrication errors in primary structural framing. Cutting will be permitted only on secondary members that are not under stress, as acceptable to the SDR. Finish gas-cut sections equal to a sheared appearance when permitted. Comply with NFPA 51B for cutting processes. 
F. Touch-Up Painting: Immediately after erection; clean field welds, bolted connections, and abraded areas of shop paint. Apply paint to exposed areas using same material as used for shop painting.

Apply by brush or spray to provide minimum dry film thickness of 3.0 mils.

\subsection{QUALITY CONTROL}

A. Sandia National Laboratories (SNL) may engage an independent testing and inspection agency to inspect high-strength bolted connections and welded connections and to perform tests and prepare test reports.

1. Testing agency shall conduct and interpret tests, state in each report whether test specimens comply with requirements, and specifically state any deviations therefrom.

2. Provide access for testing agency to places where structural steel work is being fabricated or produced so required inspection and testing can be accomplished.

3. Testing agency may inspect structural steel at plant before shipment. SNL reserves the right, at any time before final acceptance, to reject materials not complying with specified requirements.

B. Correct Deficiencies in structural steel work that inspections and laboratory test reports have indicated are not in compliance with requirements. Perform additional tests, at Contractor's expense, as necessary to reconfirm any noncompliance of original work and to show compliance of corrected work.

C. Shop-Bolted and Field-Bolted Connections: Inspect or test in accordance with AISC Specifications.

Verify that gaps of installed direct tension indicators are less than gaps specified in ASTM F959, Table 2.

D. Shop Welding and Field Welding: Inspect and test during fabrication for shop welding and during erection for field welding, of structural steel assemblies, as follows:

1. Certify welders and conduct inspections and tests as required. Record types and locations of defects found in work. Record work required and performed to correct deficiencies.

2. Perform visual inspection of all welds. 
3. Perform tests up to and including $100 \%$ of welds at SNL's option. Inspection procedures may include the following:

a. Liquid Penetrant Inspection: ASTM E165.

b. Magnetic Particle Inspection: ASTM E709; performed on root pass and on finished weld. Cracks or zones of incomplete fusion or penetration are not acceptable.

c. Radiographic Inspection: ASTM E94 and ASTM E142; minimum quality level "2-2T".

d. Ultrasonic Inspection: ASTM E164.

4. Acceptance criteria shall be as specified in AWS D1.1.

END OF SECTION 05120 


\section{STANDARD SPECIFICATION}

SECTION 05500

\section{METAL FABRICATIONS}

\section{PART 1 - GENERAL}

\subsection{DESCRIPTION OF WORK}

A. This section includes the following metal fabrications:

Rough hardware

Ladders

Ladder safety cages

Loose bearing and leveling plates

Loose steel lintels

Miscellaneous framing, supports and trim

Shelf and ledger angles

Metal bar gratings

Floor plates

Tread plates

Steel pipe guardrails and handrails

Cast nosings and treads

Metal stairs

Pipe bollards

B. Related Sections: Refer to Division 5, Section "Structural Steel" for structural steel framing components.

\subsection{REFERENCES}

A. Aluminum Association (AA)

B. American National Standards Institute (ANSI)

A14.3 Safety Requirements for Fixed Ladders

C. American Society of Testing and Materials (ASTM)

A27 Specification for Steel Castings, Carbon, for General Application

A36 Specification for Structural Steel

A47 Specification for Ferritic Malleable Iron Castings

A48 Specification for Gray Iron Castings

A53 Specification for Pipe, Steel, Black and Hot-Dipped, Zinc-Coated Welded and Seamless 
A123 Specification for Zinc (Hot-Dip Galvanized) Coatings on Iron and Steel Products

A153 Specification for Zinc Coating (Hot-Dip) on Iron and Steel Hardware

A167 Specification for Stainless and Heat-Resisting Steel Bars and Shapes

A276 Specification for Stainless and Heat-Resisting Steel Bars and Shapes

A307 Specification for Carbon Steel Bolts and Studs, 60,000 PSI Tensile Strength

A366 Specification for Steel, Sheet, Carbon, Cold-Rolled, Commercial Quality

A446 Specification for Steel Sheet, Zinc-Coated (Galvanized) by the Hot-Dip Process, Structural (Physical) Quality

A500 Specification for Cold-Formed Welded and Seamless Carbon Steel Structural Tubing in Rounds and Shapes

A510 Specification for General Requirements for Wire Rods and Coarse Round Wire, Carbon Steel

A526 Specification for Steel Sheet, Zinc-Coated (Galvanized) by the Hot-Dip Process, Commercial Quality

A569 Specification for Steel, Carbon (0.15 Maximum, Percent), HotRolled Sheet and Strip, Commercial Quality

A570 Specification for Steel, Sheet and Strip, Carbon, Hot-Rolled, Structural Quality

A780 Practice for Repair of Damaged and Uncoated Areas of Hot-Dip Galvanized Coatings

A786 Specification for Rolled Steel Floor Plates

B221 Specification for Aluminum and Aluminum-Alloy Extruded Bars, Rods, Wire, Shapes, and Tubes

B632 Specification for Aluminum-Alloy Rolled Tread Plate

C1107 Specification for Packaged Dry, Hydraulic-Cement Grout (Nonshrink)

E985 Specification for Permanent Metal Railing Systems and Rails for Building

D. American Welding Society (AWS) 
D1.1 Structural Welding Code - Steel

D1.3 Structural Welding Code - Sheet Steel

E. Code of Federal Regulations (CFR)

Title 29 Part 1910 Labor-Occupational Safety and Health Standards

F. Corps of Engineers (CE)

CRD C621 Specification for Nonshrink Grout

G. Federal Specifications (FS)

FF-B-561 Bolts, Screw, Lag

FF-B-588 Bolt, Toggle, and Expansion Sleeve, Screw

FF-S-92 Screw, Machine, Slotted, Cross-Recessed or Hexagon Head

FF-S-111 Screw, Wood

FF-S-325 Shield, Expansion; Nail, Expansion; and Nail, Drive Screw (Devices, Anchoring, Masonry)

FF-W-84 Washers, Lock (Spring)

FF-W-92 Washer, Flat (Plain)

TT-P-664 Primer Coating, Alkyd, Corrosion-Inhibiting, Lead and Chromate Free, VOC-Compliant

TT-P-645 Primer, Paint, Zinc Chromate, Alkyd Type

H. Military Standardization Documents (MIL)

P-21035 Paint, High Zinc Dust Content, Galvanizing Repair

I. National Association of Architectural Metal Manufacturers (NAAMM)

AMP $500 \quad$ Metal Finishes Manual

AMP $510 \quad$ Metal Stair Manual

MBG 531 Metal Bar Grating Manual

MBG 532 Heavy Duty Metal Bar Grating Manual

Pipe Railing Manual

J. National Fire Protection Association (NFPA) 
K. Steel Structures Painting Council (SSPC)

SSPC-PA 1 Paint Application Specification No. 1

SSPC Paint 12 Paint Specification No. 12 Cold Applied Asphalt Mastic (Extra Thick Film)

SSPC Paint 20 Paint Specification No. 20 Zinc-Rich Primers (Type I, "Inorganic," and Type II, "Organic")

SSPC-SP1 Surface Preparation Specification No. 1 "Solvent Cleaning"

SSPC-SP2 Surface Preparation Specification No. 2 "Hand-Tool Cleaning"

SSPC-SP3 Surface Preparation Specification No. 3 "Power Tool Cleaning"

SSPC-SP7 Surface Preparation Specification No. 7 "Brush-Off Blast Cleaning"

L. Uniform Building Code (UBC)

M. Uniform Federal Accessibility Standards (UFAS)

Fed. Std. 795

\subsection{DEFINITIONS}

A. Refer to ASTM E985 for railing-related terms that apply to this section.

B. Refer to the NAAMM publications listed in Article 1.02 "References" for definition of terms that apply to this section.

\subsection{SUBMITTALS}

General: Submit the following in accordance with Conditions of Contract and Division 1, Section "Descriptive Submittals".

A. Product Data: Submit product data for products used in metal fabrications, including paint products, grout and fasteners.

B. Shop Drawings: Submit detailed shop and erection drawings of each metal fabrication indicated. Include plans, elevations, sections, and details of metal fabrications and their connections. Show anchorage and accessory items.

C. Welder certificates signed by Contractor certifying that welders comply with requirements specified in $1.05 \mathrm{C}$.

D. Qualification data for firm specified in 1.05B to demonstrate their capabilities and experience. 


\subsection{QUALITY ASSURANCE}

A. Codes and Standards: Comply with provisions of the following, except as otherwise indicated. Where conflicts occur, comply with the more stringent requirements.

1. ANSI 14.3

2. AWS D1.1 and D1.3

3. NFPA 101

B. Fabricator Qualifications: Firm experienced in successfully producing metal fabrications similar to that shown on the drawings, with sufficient production capacity to produce required units without causing delay in the work.

C. Qualify welding processes and welding operators in accordance with AWS D1.1 and D1.3.

Certify that each welder has satisfactorily passed AWS qualification tests for welding processes involved and, if pertinent, has undergone recertification.

D. All materials used shall be free of lead, and asbestos fibers.

E. Use of damaged items is prohibited except by specific authorization of Sandia Delegated Representative (SDR) in writing.

1.06 DELIVERY. STORAGE. AND HANDLING

A. Deliver materials to the job site in good condition and properly protected against damage to finished surfaces.

B. Storage on Site: Store materials in a location and in a manner to avoid damage. Stacking shall be done in a way which will prevent bending.

Store metal components and materials in a clean, dry location. Cover with waterproof paper, tarpaulin or polyethylene sheeting in a manner that will permit circulation of air inside the cover.

C. Keep handling on-site to a minimum. Exercise care to avoid damage to finishes of material.

\subsection{PROJECT CONDITIONS}

Field Measurements: Check actual locations of walls and other construction to which metal fabrications must fit, by accurate field measurements before fabrication; show recorded measurements on final shop drawings. Coordinate fabrication schedule with construction progress to avoid delay-of work. 
Where field measurements cannot be made without delaying the work, guarantee dimensions and proceed with fabrication of products without field measurements. Coordinate construction to ensure that actual opening dimensions correspond to guaranteed dimensions. Allow for trimming and fitting.

\subsection{SEQUENCING AND SCHEDULING}

Sequence and coordinate installation of wall handrails as follows:

A. Mount handrails only on completed walls. Do not support handrails temporarily by any means not satisfying structural performance requirements.

B. Mount handrails only on gypsum board assemblies reinforced with proper blocking and backing to receive anchors, and where the location of concealed anchor plates has been clearly marked for benefit of Installer.

\section{PART 2 - PRODUCTS}

\subsection{FERROUS METALS}

A. Metal Surfaces. General: Form metal fabrications exposed to view upon completion of the work, provide materials selected for their surface flatness, smoothness, and freedom from surface blemishes. Do not use materials whose exposed surfaces exhibit pitting, seam marks, roller marks, rolled trade names, roughness, and, for steel sheet, variations in flatness exceeding those permitted by referenced standards for stretcher-leveled sheet.

B. Steel Plates. Shapes. and Bars: ASTM A36.

C. Rolled Steel Floor Plates: ASTM A786.

D. Steel Bars for Gratings: ASTM A569 or ASTM A36.

E. Wire Rod for Grating Cross Bars: ASTM A510.

F. Steel Tubing: Cold-formed, ASTM A500, Grade B, unless otherwise indicated.

G. Uncoated Structural Steel Sheet: Hot-rolled, ASTM A570, Grade 30 unless otherwise indicated.

H. Uncoated Steel Sheet (Commercial quality): Cold-rolled, ASTM A366.

I. Galvanized Steel Sheet: Quality as follows:

1. Structural Quality: ASTM A446; Grade A, unless another grade required for design loading, and G90 coating designation unless otherwise indicated. 
2. Commercial Quality: ASTM A526, G90 coating designation unless otherwise indicated.

J. Steel Pipe: ASTM A53, Type S, Grade B, standard weight (schedule 40), black finish, unless otherwise indicated.

K. Gray Iron Castings: ASTM A48, Class 30.

L. Malleable Iron Castings: ASTM A47, Grade 32510.

M. Brackets. Flanges and Anchors: Cast or formed metal of the same type material and finish as supported rails, unless otherwise indicated.

N. Concrete Inserts: Threaded or wedge type; galvanized ferrous castings, either malleable iron, ASTM A47, or cast steel, ASTM A27. Provide bolts, washers, and shims as required, hot-dip galvanized per ASTM A153.

O. Welding Rods and Bare Electrodes: Select in accordance with AWS specifications for the metal alloy to be welded.

\subsection{STAINLESS STEEL}
A. Bar Stock: ASTM A276, Type 302 or 304.
B. Plate: ASTM A167, Type 302 or 304.

2.03 Aluminum

A. Extruded Bars and Shapes: ASTM B221, alloy 6061-T6 or 6063-T6 for bearing bars of gratings and shapes and 6061-T6 for grating cross bars.

B. Aluminum-Alloy Rolled Tread Plate: ASTM B632, alloy 6061-T4 for treads and 6061-T6 for platforms.

\subsection{GROUT}

Nonshrink Nonmetallic Grout: Premixed, factory-packaged, nonstaining, noncorrosive, nongaseous grout complying with ASTM C1107 (formerly referenced as CE CRD C621). Provide grout specifically recommended by manufacturer for interior and exterior applications of type specified in this section.

Subject to compliance with requirements, products that may be incorporated in the work include, but are not limited to the following:

Euco N-S Grout - Euclid Chemical Co.

Kemset - Chem-Masters Corp.

Crystex - L \& M Construction Chemicals, Inc.

Sonogrout - Sonneborn Building Products Div., Rexnord Chemical Products, Inc.

Supreme Grout - Cormix, Inc.

Sure-Grip High Performance Grout - Dayton Superior 
Vibropruf \#11 - Lambert Corp.

\subsection{FASTENERS}

A. General: Provide zinc-coated fasteners for exterior use or where built into exterior walls. Select fasteners for the type, grade, and class required. Suspect/counterfeit bolts will not be accepted and will be replaced at Contractor's expense.

B. Bolts and Nuts: Regular hexagon head type, ASTM A307, Grade A.

C. Lag Bolts: Square head type, FS FF-B-561.

D. Machine Screws: FS FF-S-92.

E. Wood Screws: Flat head carbon steel, FS FF-S-111.

F. Plain Washers: Round, carbon steel, FS FF-W-92.

G. Expansion Anchors: Expansion anchors complying with FS FF-S-325.

H. Toggle Bolts: Tumble-wing type, FS FF-B-588, type, class, and style as required.

I. Lock Washers: Helical spring type carbon steel, FS FF-W-84.

J. Special Fasteners: Split nuts, indicator washers and others, as indicated.

\subsection{PAINT}

A. Shop Primer for Ferrous Metal: Red oxide, lead- and cadmium-free, corrosion-inhibiting primer complying with performance requirements of $F S$ TT-P-664.

B. Galvanizing Repair Paint: High zinc dust content paint for regalvanizing welds in galvanized steel, with dry film containing not less than $94 \%$ zinc dust by weight, and complying with MIL-P-21035 or SSPC-Paint-20.

C. Bituminous Paint: Cold-applied asphalt mastic complying with SSPCPaint-12 except containing no asbestos fibers.

D. Zinc Chromate Primer: FS TT-P-645.

\subsection{CONCRETE FILL}

A. Concrete Materials and Properties: Comply with requirements for Division 3, Section "Cast-in-Place Concrete" for normal weight, ready-mix concrete with minimum 28-day compressive strength of $3000 \mathrm{psi}(21 \mathrm{MPa})$, unless higher strength indicated. 
B. Nonslip Aggregate Finish: Factory-graded, packaged material containing fused aluminum oxide grits or crushed emery as abrasive aggregate; rustproof and nonglazing; unaffected by freezing, moisture, or cleaning

\subsection{FABRICATION. GENERAL}

A. Form metal fabrications from materials of size, thickness, and shapes indicated but not less than that needed to comply with performance requirements indicated. Work to dimensions indicated or accepted on shop drawings, using proven details of fabrication and support. Use type of materials indicated or specified for various components of each metal
fabrication.

B. Form exposed work true to line and level with accurate angles and surfaces

C. Allow för thermal movement resulting from the following maximum change (range) in ambient temperature in the design, fabrication, and installation of installed metal assemblies to prevent buckling, opening up of joints, and overstressing of welds and fasteners. Base design calculations on actual surface temperatures of metals due to both solar heat gain and nighttime sky
heat loss.

Temperature change (Range): $100 \mathrm{deg} F(38 \mathrm{deg}$ C).

D. Shear and punch metals cleanly and accurately. Remove burrs.

E. Ease exposed edges to a radius of approximately $1 / 32$ inch $(0.794 \mathrm{~mm})$, unless otherwise indicated. Form bent-metal corners to smallest radius possible without causing grain separation or otherwise impairing work.

F. Remove sharp or rough areas on exposed traffic surfaces.

G. Weld corners and seams continuously to comply with AWS recommendations and the following: 1. Use materials and methods that minimize distortion and develop strength
and corrosion resistance of base metals.

2. Obtain fusion without undercut or overlap.

3. Remove welding flux immediately.

4. At exposed connections, finish exposed welds and surfaces smooth and blended so that no roughness shows after finishing and contour of welded surface matched those adjacent.

H. Form exposed connections with hairline joints, flush and smooth, using concealed fasteners wherever possible. Use exposed fasteners of type boits. Locate joints where least conspicuous. 
I. Provide for anchorage of type indicated; coordinate with supporting structure. Fabricate and space anchoring devices to provide adequate support for intended use.

J. Shop Assembly: Preassemble items in shop to greatest extent possible to minimize field splicing and assembly. Disassemble units only as necessary for shipping and handling limitations. Use connections that maintain structural value of joined pieces. Clearly mark units for reassembly and coordinated installation.

K. Cut. reinforce. drill and tap miscellaneous metal work as indicated to receive finish hardware, screws, and similar items.

L. Fabricate joints that will be exposed to weather in a manner to exclude water, or provide weep holes where water may accumulate.

\subsection{ROUGH HARDWARE}

Furnish bent or otherwise custom fabricated bolts, plates, anchors, hangers, dowels, and other miscellaneous steel and iron shapes as required. Fabricate items to sizes, shapes, and dimensions required.

\subsection{STEEL LADDERS}

A. General: Fabricate ladders for the locations shown, with dimensions, spacings, details and anchorages as indicated. Comply with requirements of ANSI A14.3 and CFR 29 1910.27; where conflicts occur, comply with the more stringent requirements.

B. Side Rails: Continuous steel flat bars, minimum size (cross section) 2-1/2" x $3 / 8$ " $(63.5 \mathrm{~mm} \times 9.5 \mathrm{~mm})$, with eased edges, spaced 18 inches $(0.46 \mathrm{~m})$ apart.

For ladders subject to unusually corrosive atmospheric exposures, steel flat bars 2-1/2" x 1/2" (63.5 mm x $12.7 \mathrm{~mm})$.

C. Bar Rungs: Round steel bars, minimum 3/4 inch $(19.1 \mathrm{~mm})$ diameter, spaced no greater than 12 inches $(305 \mathrm{~mm})$ o.c. and uniform throughout the length of the ladder.

Individual metal rungs embedded in concrete which serve as access to pits and other areas under floors, should have a minimum diameter of 1 inch $(25 \mathrm{~mm})$ or shall otherwise be treated to resist corrosion and rusting.

D. Fit rungs in centerline of side rails, plug weld and grind smooth on outer rail faces.

E. Support each ladder at top and bottom and at intermediate points spaced not more than 5'-0" $(1.5 \mathrm{~m})$ 0.c. by means of welded brackets, unless otherwise indicated. 
1. Size brackets to support design dead and live loads indicated and to hold centerline of ladder rungs clear of the wall surface by not less than 7 inches $(178 \mathrm{~mm})$.

2. Extend side rails 42 inches $(1.1 \mathrm{~m})$ above top rung, and return rails to wall or structure unless other secure handholds are provided. If the adjacent structure does not extend above the top rung, goose-neck the extended rails back to the structure to provide secure ladder access.

\subsection{LADDER SAFETY CAGES}

A. General: Fabricate ladder safety cages to comply with requirements of ANSI A14.3 and CFR 29 1910.27; where conflicts occur, comply with the more stringent requirements. Assemble safety cages by welding.

B. Primary Hoops: Steel bars, 3" x 1/4" (76 $\mathrm{mm} \times 6.4 \mathrm{~mm}$ ), for top, bottom, and for cages longer than 20 feet $(6.1 \mathrm{~m})$, intermediate hoops spaced not more than $20^{\prime}-0^{\prime \prime}(6.1 \mathrm{~m})$ o.c.

C. Secondary Intermediate Hoops: Steel bars, 2" x 1/4" (51 mm x $6.4 \mathrm{~mm})$ hoops spaced not more than 4'-0" $(1.2 \mathrm{~m})$ o.c. between primary hoops.

D. Vertical Bars: Steel bars, 1-1/2" x 1/4" (38.1 mm x $6.4 \mathrm{~m})$, secured to each hoop, spaced at intervals not more than $40^{\circ}(0.7 \mathrm{rad})$ o.c. around the circumference of the cage, maximum spacing of approximately 9-1/2 inches $(241 \mathrm{~mm})$ o.c.

E. Fasten assembled safety cage to ladder rails and adjacent construction as indicated.

\subsection{LOOSE BEARING AND LEVELING PLATES}

Provide loose bearing and leveling plates for steel items bearing on masonry or concrete construction, made flat, free from warps or twists, and of required thickness and bearing area. Drill plates to receive anchor bolts and for grouting as required.

\subsection{LOOSE STEEL LINTELS}

A. Fabricate loose structural steel lintels from steel angles and shapes of size indicated for opening and recesses in masonry walls and partitions at locations indicated.

B. Weld adjoining members together to form a single unit where indicated.

C. Size loose lintels for equal bearing of one inch per foot of clear span but not less than 8 inches $(203 \mathrm{~mm})$ bearing at each side of openings, unless otherwise indicated.

\subsection{MISCELLANEOUS METAL ITEMS}


A. Miscellaneous Framing and Supports: Provide steel framing and supports for applications indicated which are not a part of structural steel framework, as required to complete work.

1. Fabricate units to sizes, shapes, and profiles indicated and required to receive adjacent other construction retained by framing and supports. Fabricate from structural steel shapes, plates, and steel bars of welded construction using mitered joints for field connection. Cut, drill, and tap units to receive hardware, hangers, and similar items.

2. Equip units with integrally welded anchors for casting into concrete or building into masonry. Furnish inserts if units must be installed after concrete is placed.

Except as otherwise indicated, space anchors 24 inches $(61 \mathrm{~cm})$ o.c. and provide minimum anchor units in the form of steel straps 1-1/4" wide $\times 1 / 4$ " $\times 8$ " long ( $31.8 \mathrm{~mm} \times 6.4 \mathrm{~mm} \times 203 \mathrm{~mm}$ ).

B. Miscellaneous Steel Trim: Provide shapes and sizes indicated for profiles shown. Unless otherwise indicated, fabricate units from structural steel shapes, plates, and steel bars, with continuously welded joints and smooth exposed edges. Use concealed field splices wherever possible. Provide cutouts, fittings, and anchorages as required for coordination for assembly and installation with other work.

\subsection{SHELF AND LEDGER ANGLES}

A. Fabricate shelf and ledger angles from steel angles of sizes indicated and for attachment to concrete framing. Provide slotted holes to receive $3 / 4$ inch $(19.1 \mathrm{~mm})$ bolts, spaced not more than 6 inches $(152 \mathrm{~mm})$ from ends and not more than 24 inches $(0.61 \mathrm{~m})$ o.c., unless otherwise indicated.

B. For cavity walls, provide vertical channel brackets to support shelf/ledger angles from back-up masonry and concrete. Align expansion joints in angles with indicated expansion joints in cavity wall exterior wythe.

C. Furnish wedge-type concrete inserts, complete with fasteners, for attachment of shelf angles to cast-in-place concrete.

\subsection{METAL BAR GRATINGS}

A. General: Produce metal bar gratings of description indicated per NAAMM marking system that comply with the following:

1. Metal Bar Grating Standard: "Standard Specifications for Metal Bar Grating and Metal Bar Grating Treads" published in ANSI/NAAMM MBG 531 .

2. Heavy Duty Metal Bar Grating Standard: "Guide Specifications for Heavy Duty Metal Bar Grating" published in ANSI/NAAMM MBG 532. 
B. If gratings are located in walking surfaces, gratings shall have spaces no greater than $1 / 2$ inch $(12.7 \mathrm{~mm})$ wide in one direction.

If gratings have elongated openings, then they shall be placed so that the long dimension is perpendicular to the dominant direction of travel.

C. Fabricate steel gratings to comply with requirements as indicated on the drawings.

D. Traffic Surface for Steel and Aluminum Bar Gratings: Plain, unless otherwise indicated.

E. Steel Finish: Shop prime paint applied in accordance with manufacturer's standard practice.

F. Aluminum Finish: Mill (as fabricated), unless otherwise indicated.

G. Fabricate removable grating sections with banding bars attached by welding to entire perimeter of each section. Include anchors and fasteners of type indicated, or if not indicated, as recommended by manufacturer, for attachment to supports.

1. Heavy Duty Grating: Provide not less than 4 anchor blocks, 1/4 inch $(6.4 \mathrm{~mm})$ minimum thickness, for each section of grating composed of bearing bars over $3 / 16$ inch $(4.8 \mathrm{~mm})$ in thickness, with each block shopwelded to 2 bearing bars.

2. Nonheavy Duty Grating: Provide not less than 4 saddle clips for each grating section composed of rectangular bearing bars $3 / 16$ inch $(4.8 \mathrm{~mm})$ or less in thickness and spaced not less than $15 / 16$ inch $(23.8 \mathrm{~mm}$ ) o.c., with each clip designed and fabricated to fit over 2 bearing bars.

Furnish threaded bolts with nuts and washers for each clip required, unless otherwise indicated.

H. Attach toe plates to grating by welding, unless otherwise indicated.

Toe plate height: 4 inches $(102 \mathrm{~mm})$, unless a greater height indicated.

I. Fabricate cutouts in grating sections for penetrations indicated. Arrange layout of cutouts to permit grating removal without disturbing items penetrating gratings.

1. Edge band openings in grating that interrupt 4 or more bearing bars with bars of same size and material as bearing bars.

2. Do not notch bearing bars at supports to maintain elevation.

J. Available Manufacturers: Subject to compliance with requirements, manufacturers offering metal bar gratings that may be incorporated in the Work include, but are not limited to, the following:

Alabama Metal Industries Corp.

Barnet/Bates Corp. 
IKG Industries

Klemp Corp.

Ohio Gratings, Inc.

Seidelhuber Metal Products, Inc.

\subsection{FLOOR PLATE}

A. Fabricate raised pattern floor plates from rolled steel floor plate of thickness and pattern indicated. If not indicated, pattern as selected from manufacturer's standard patterns. See ASTM A786 for pattern illustrations.

B. Abrasive Surface Floor Plate: Manufacturer's standard abrasive granules, rolled into surface of steel plate, where indicated.

C. Include steel angle stiffeners and fixed and removable sections as indicated.

Provide 2 steel bar drop handles for lifting plates, one at each end of each removable section.

\subsection{TREAD PLATE}

A. Fabricate raised pattern tread plates from aluminum-alloy rolled tread plate in pattern and thickness as indicated. See ASTM B632 for pattern illustrations.

B. Abrasive Surface Floor Plate: Manufacturer's standard abrasive granules, rolled into surface of aluminum plate, where indicated.

C. Include aluminum angle stiffeners and fixed and removable sections as indicated.

Provide 2 aluminum bar drop handles for lifting plates, one at each end of each removable section.

\subsection{STEEL PIPE GUARDRAILS AND HANDRAILS}

A. General: Fabricate pipe guardrails and handrails to comply with requirements indicated for dimensions, details, finish, and member sizes, including wall thickness of pipe, post spacings, and anchorage.

B. Interconnect guardrails and handrail members by butt-welding or welding with internal connectors, at fabricator's option, unless otherwise indicated.

At tee and cross intersections, cope ends of intersecting members to fit contour of pipe to which end is joined, weld all around and grind smooth.

C. Form changes in directions of railing members as follows:

1. By use of welded prefabricated steel elbow fittings. 
2. By bending, of radius indicated.

3. By mitering at elbow bends.

D. Form simple and compound curves by bending pipe in jigs to produce uniform curvature for each repetitive configuration required; maintain cylindrical cross-section of pipe throughout entire bend without buckling, twisting, cracking, or otherwise deforming exposed surfaces of pipe.

E. Provide wall returns at ends of wall-mounted handrails, unless otherwise indicated.

F. Close exposed ends of pipe by welding $3 / 16$ inch $(4.8 \mathrm{~mm})$ thick steel plate in place or by use of prefabricated fittings, except where clearance of end of pipe and adjoining wall surface is $1 / 4$ inch $(6.4 \mathrm{~mm})$ or less.

G. Toe Boards: Where indicated, provide toe boards at railings around openings and at the edge of open-sided floors and platforms. Fabricate to dimensions and details indicated, or if not indicated, use 4 inches high $\times 1 / 4$ inch (102 mm x $6.4 \mathrm{~mm}$ ) steel bar welded to each railing post.

H. Brackets. Flanges. Fittings, and Anchors: Provide wall brackets, end closures, flanges, miscellaneous fittings, and anchors for interconnections of pipe and attachment of guardrails and handrails to other work. Furnish inserts and other anchorage devices for connecting guardrails and handrails to concrete or masonry work.

1. For railing posts set in concrete, fabricate sleeves from steel pipe not less than 6 inches $(152 \mathrm{~mm})$ long and with an inside diameter not less than $1 / 2$ inch $(12.7 \mathrm{~mm})$ greater than the outside diameter of post, with steel plate closure welded to bottom of sleeve.

2. For removable railing posts, fabricate slip-fit sockets from steel pipe whose inside diameter is sized for a close fit with posts and to limit deflection of post without lateral load, measured at top, to not more than $1 / 12$ of post height. Provide socket covers designed and fabricated to resist accidental dislodgement.

I. Fillers: Provide steel sheet or plate fillers of thickness and size indicated or required to support structural loads of handrails where needed to transfer wall bracket loads through wall finishes to structural supports. Size fillers to suit wall finish thicknesses. Size fills to produce adequate bearing to prevent bracket rotation and overstressing of substrate.

\subsection{CAST NOSINGS AND TREADS}

A. Fabricate units of material, sizes, and configurations indicated. If not indicated, provide cast-iron units with integral abrasive finish. Furnish in lengths as required to accurately fit each opening or conditions.

Cast units with an integral abrasive grit consisting of aluminum oxide, silicone carbide, or a combination of both. 
B. Available Manufacturers: Subject to compliance with requirements, manufacturers offering products that may be incorporated in the Work include, but are not limited to, the following:

AMSTEP Products, division of American Safety Technologies, Inc. American Safety Tread Co., Inc.

IKG Industries

Safe-T-Metal Co., Inc.

Wooster Products Inc.

C. Provide anchors for embedding units in concrete, either integral or applied to units, as standard with the manufacturer.

D. Apply black asphaltic coating to concealed bottoms, sides, and edges of cast-iron units set into concrete.

\subsection{STEEL FRAMED STAIRS}

A. General: Construct stairs to conform to sizes and arrangements indicated with welded connections, unless otherwise indicated. Provide complete stair assemblies, including metal framing, hangers, columns, railings, newels, balusters, struts, clips, brackets, bearing plates, and other components necessary for the support of stairs and platforms, and as required to anchor and contain the stairs on the supporting structure.

Fabricate treads and platforms of exterior stairs to accommodate slopes to drain in finished traffic surfaces.

B. Stair Framing: Fabricate stringers of structural steel channels, or plates, or a combination thereof, as indicated. Provide closures for exposed ends of stringers. Construct platforms of structural steel channel headers and miscellaneous framing members as indicated. Bolt or weld headers to stringers, newels, and framing members to stringers and headers; fabricate and join so that bolts, if used, do not appear on finish surfaces.

C. Metal Pan Risers. Subtreads. and Subplatforms: Shape metal pans for risers and subtreads to conform to configuration shown. Provide thickness of structural steel sheet for metal pans as indicated.

1. Form metal pans of uncoated cold-rolled steel sheet, unless otherwise indicated.

2. Form metal pans of galvanized steel sheet, where indicated on the drawings.

3. Attach Risers and Subtreads to Stringers by the following methods:

a. Directly weld risers and subtreads to stringers; locate welds on side of metal pans to be concealed by concrete fill.

b. Attach by means of brackets made of steel angles or bars. Weld brackets to stringers and attach metal pans to brackets by welding or bolting. 
4. Provide subplatforms of configuration and construction shown, with thickness of structural steel sheet as indicated. Attach subplatform to platform framing members with welds or as otherwise indicated.

Construct subplatforms with smooth soffits.

D. Steel Floor Plate Treads and Platforms: Provide raised pattern steel floor plate in pattern indicated or, if not indicated, as selected from manufacturer's standard patterns.

1. Form treads of $1 / 4$ inch $(6.4 \mathrm{~mm})$ thick raised pattern steel floor plate with integral nosing and back edge stiffener. Weld steel supporting brackets to stringers and treads to brackets.

2. Fabricate platforms of raised pattern steel floor plate of thickness indicated. Provide nosing matching that on treads at all landings. Secure to platform framing members with welds.

E. Floor Grating Treads and Platforms: Provide patterns, spacing, and bar sizes indicated; fabricate to comply with NAAMM MBG 531.

1. Finish to be shop prime paint, unless otherwise indicated.

2. Fabricate grating treads with steel plate nosing on one edge and with steel angle or steel plate carrier at each end for stringer connections. Secure treads to stringers with bolts or as otherwise indicated.

3. Fabricate grating platforms, with nosing matching that on grating treads, at all landings. Provide toe plates at open-sided edges of grating platform. Secure grating to platform frame with welds or as otherwise indicated.

F. Stair Guardrails and Handrails: Comply with applicable requirements specified elsewhere in this section for steel pipe guardrails and handrails, and as follows:

1. Fabricate newels of steel tubing and provide newel caps of gray-iron castings, as shown.

2. Railings may be bent at corners, rail returns, and wall returns, instead of using prefabricated fittings.

3. Connect railing posts to stair framing by direct welding, unless otherwise indicated.

\subsection{PIPE BOLLARDS}

Fabricate pipe bollards from 4-inch (102 $\mathrm{mm}$ ) standard black steel pipe, Schedule 40 , unless otherwise indicated. Cap bollards with $1 / 4$ inch $(6.4 \mathrm{~mm})$ minimum thickness steel base plate, or as otherwise indicated.

\section{$2.23 \quad$ FINISHES}


A. General: Comply with NAAMM AMP 500 "Metal Finishes Manual" for recommendations relative to application and designations of finishes.

B. Finish metal fabrications after assembly.

\subsection{STEEL AND IRON FINISHES}

A. General: Shop-paint uncoated surfaces of metal fabrications, except those to be embedded in concrete or masonry or to receive sprayed-on fireproofing, surfaces and edges to be welded, and galvanized surfaces, unless otherwise indicated. Comply with requirements of SSPC-PA 1 for shop painting.

B. Galvanizing: For those items indicated for galvanizing, apply zinc-coating by the hot-dip process in compliance with the following requirements:

1. ASTM A123 for galvanizing both fabricated and unfabricated iron and steel products made of uncoated rolled, pressed, and forced shapes, plates, bars, and strip 0.0299 inch $(0.7595 \mathrm{~mm})$ thick and heavier.

2. ASTM A153 for galvanizing iron and steel hardware.

C. Surface Preparation for Shop Priming: Prepare uncoated ferrous metal surfaces to comply with minimum requirements indicated below.

1. Remove oil, grease and similar contaminants in accordance with SP-1, "Solvent Cleaning".

2. Remove loose rust, scale, spatter, slag and other deleterious materials in accordance with SSPC, utilizing the following methods as required:

SP-2 "Hand-Tool Cleaning"

SP-3 "Power-Tool Cleaning"

SP-7 "Brush-Off Blast Cleaning"

D. Painting: Immediately after surface preparation, apply structural steel primer paint in accordance with manufacturer's instructions and at a rate to provide dry film thickness of not less than 3.0 mils $(0.076 \mathrm{~mm})$. Use painting methods that result in full coverage of joints, corners, edges, and exposed surfaces.

Apply 2 coats of paint to surfaces that are inaccessible after assembly or erection.

\subsection{ALUMINUM FINISH}

Mill (as fabricated) finish, unless otherwise indicated.

PART 3 - EXECUTION

3.01 examination 
Installer shall examine the areas and conditions under which metal fabrication items are to be installed. Notify the SDR in writing of conditions detrimental to the proper and timely completion of the work. Do not proceed with the work until unsatisfactory conditions have been corrected in a manner acceptable to the Installer and SDR.

\subsection{PREPARATION}

A. Coordinate and furnish anchorages, setting drawings, diagrams, templates, instructions, and directions for installation of anchorages, including concrete inserts, sleeves, anchor bolts, and miscellaneous items having integral anchors that are to be embedded in concrete or masonry construction. Coordinate delivery of such items to project site.

B. Center nosings on tread widths with noses flush with riser faces and tread surfaces.

C. Set sleeves in concrete with tops flush with finish surface elevations; protect sleeves from water and concrete entry.

\subsection{INSTALLATION. GENERAL}

A. Fastening to In-Place Construction: Provide anchorage devices and fasteners where necessary for securing miscellaneous metal fabrications to in-place construction; include threaded fasteners for concrete and masonry inserts, toggle bolts, through-bolts, lag bolts, wood screws and other connectors as required.

B. Cutting, Fitting and Placement: Perform cutting, drilling, and fitting required for installation of miscellaneous metal fabrications. Set metal fabrication accurately in location, alignment, and elevation; with edges and surfaces level, plumb, true, and free of rack; and measured from established lines and levels.

C. Provide temporary bracing or anchors in formwork for items that are to be built into concrete masonry or similar construction.

D. Fit exposed connections accurately together to form hairline joints. Weld connections that are not to be left as exposed joints, but cannot be shopwelded because of shipping size limitations. Do not weld, cut, or abrade the surfaces of exterior units which have been hot-dip galvanized after fabrication, and are intended for bolted or screwed field connections.

E. Field Welding: Comply with AWS Code for procedures of manual shielded metal-arch welding, appearance and quality of welds made, methods used in correcting welding work, and the following:

1. Use materials and methods that minimize distortion and develop strength and corrosion resistance of base metals.

2. Obtain fusion without undercut or overlap. 
3. Remove welding flux immediately.

4. At exposed connections, finish exposed welds and surfaces smooth and blended so that no roughness shows after finishing and contour of welded surfaces matches those adjacent.

F. Grout: Follow manufacturer's recommendations for substrate preparation and application.

G. Corrosion Protection: Coat concealed surfaces of aluminum that will come into contact with grout, concrete, masonry, wood, or dissimilar metals with a heavy coat of bituminous paint or zinc chromate primer.

\subsection{SETTING LOOSE PLATES}

A. Clean concrete and masonry bearing surfaces of any bond-reducing materials, and roughen to improve bond to surfaces. Clean bottom surface of bearing plates.

B. Set loose leveling and bearing plates on wedges, or other adjustable devices. After the bearing members have been positioned and plumbed, tighten the anchor bolts. Do not remove wedges or shims, but if protruding, cut off flush with the edge of the bearing plate before packing with grout. Pack grout solidly between bearing surfaces and plates to ensure that no voids remain.

\subsection{INSTALLATION OF METAL BAR GRATINGS}

A. General: Install gratings to comply with requirements of NAAMM grating standard that apply to grating types and bar sizes indicated, including installation clearances and standard anchoring details.

B. Secure removable units to supporting members with type and size of clips and fasteners indicated, or if not indicated, as recommended by grating manufacturer for type of installation conditions shown.

C. Secure non-removable units to supporting members by welding where both materials are the same; otherwise, fasten by bolting as indicated above.

D. Attach toe plates to gratings by welding, at locations indicated.

\subsection{INSTALLATION OF STEEL PIPE GUARDRAILS AND HANDRAILS}

A. Adjust railings prior to anchoring to ensure matching alignment at abutting joints. Space posts at spacing indicated, or if not indicated, as required by design loadings. Plumb posts in each direction. Secure posts and railing ends to building construction as follows:

1. Anchor posts in concrete by means of pipe sleeves preset and anchored into concrete. After posts have been inserted into sleeves, fill annular space between post and sleeve solid with nonshrink, nonmetallic grout, 
mixed and placed to comply with anchoring material manufacturer's directions.

2. Anchor posts and rail ends to steel with welded connections, unless otherwise indicated.

3. Anchor posts and rail ends into concrete and masonry with steel round flanges welded to post and rail ends, and anchored into wall construction with expansion shields and bolts.

4. Install removable railing sections where indicated in slip-fit metal sockets cast into concrete. Accurately locate sockets to match post spacing.

B. Secure handrails to wall with wall brackets and end fittings. Provide bracket with not less than 1-1/2 inch $(38.1 \mathrm{~mm})$ clearance from inside face of handrail and finished wall surface. Locate brackets at spacing not less than $5^{\prime}-0^{\prime \prime}(1.5 \mathrm{~m})$ 0.c., unless otherwise indicated. Secure wall brackets and wall return fittings to building construction as follows:

1. Use type of bracket with flange tapped for concealed anchorage to threaded hanger bolt.

2. For concrete and solid masonry anchorage, use drilled-in expansion shield and either concealed hanger bolt or exposed lag bolt, as applicable.

3. For hollow masonry anchorage, fasten brackets directly on masonry wall using toggle bolts.

4. For steel framed gypsum board assemblies, fasten brackets to wood blocking using lag bolts or to metal blocking using self-tapping screws, of size and type required to support structural loads.

C. Expansion Joints: Provide expansion joints at locations indicated, or if not indicated, at intervals not to exceed 40 feet $(12.2 \mathrm{~m})$. Provide slip joint with internal sleeve extending 2 inches $(51 \mathrm{~mm})$ beyond joint on either side; fasten internal sleeve securely to one side; locate joint within 6 inches (152 $\mathrm{mm}$ ) of posts.

\subsection{INSTALLATION OF CAST NOSINGS AND TREADS}

Install cast nosings and treads with anchorage system indicated to comply with manufacturer's recommendations.

\subsection{INSTALLATION OF PIPE BOLLARDS}

Install bollards at locations shown on drawings. After installation, fill pipe with concrete and provide a smooth convex curve at the top of the pipe. 
A. Touch-Up Painting of Steel Items: Immediately after erection, clean field welds, bolted connections, abraded areas of shop paint, and paint exposed areas with same material as used for shop painting to comply with SSPC-PA 1 requirements for touch-up of field painted surfaces.

Apply by brush or spray to provide a minimum dry film thickness of 3.0 mils $(0.076 \mathrm{~mm})$.

B. For galvanized surfaces clean welds, bolted connections and abraded areas and apply galvanizing repair paint to comply with ASTM A780.

END OF SECTION 05500 


\section{STANDARD SPECIFICATION \\ SECTION 09900 \\ PAINTING}

\section{PART 1 - GENERAL}

\subsection{DESCRIPTION OF WORK}

A. Definition: "Paint" as used herein means all coating systems materials, including primers, emulsions, enamels, stains, sealers ant fillers, and other applied materials whether used as prime, intermediate, or finish coats. Surfaces listed in the painting schedule herein shall receive the surface preparation and number of coats prescribed.

B. Included: All exposed new work shall be painted unless excluded in Paragraph $1.01 \mathrm{C}$. or otherwise excluded. Existing work shall be painted when specified. Painting shall include:

1. Priming of damaged or abraded shop coats.

2. Those surfaces damaged and repaired by work connected with this project shall be painted unless the entire surface is otherwise shown or specified to be painted. Patch painting shall closely match the existing adjacent paint.

3. Color coding of sprinkler piping.

4. Color coding of all other Mechanical Piping Systems is limited to those items indicated in the contract documents.

5. Used full height metal partitions and patrol height metal cubicle partitions installed or relocated on this project.

6. Touch-up painting of all factory finishes damaged in shipment or installation and repainting if colors are not satisfactory.

C. Excluded: The following items shall not be painted except where otherwise noted on the drawings or in the contract documents:

1. Plated or factory-finished items, except where "factory finish" is prime coat only and except where colors are not satisfactory.

2. Roof flashing materials around roof-mounted equipment.

3. Finished metal surfaces including: Anodized aluminum, stainless steel, chromium plate, brass, and bronze. 
4. Glass.

5. Prefinished new acoustical materials.

6. Galvanized components of prefabricated metal buildings.

7. Interior and exterior concrete slabs on grade.

8. Equipment room surfaces.

\subsection{RELATED WORK}

Related work which is specified in other sections includes::

- Shop coats on fabricated items.

- Identification labels for piping.

- Taping and texturing of gypsum wallboard.

1.03 QUALITY ASSURANCE- Single Source Responsibility: Provide primers and other undercoat paint produced by same manufacturer as finish coats. Use only thinners approved by paint manufacturer, and use only within recommended limits.

\subsection{DELIVERY AND STORAGE}

A. Deliver materials to job site in original, new and unopened packages and containers bearing manufacturer's name and label, containing the following information:

- Name or title of material

- Manufacturer's stock number and date of manufacture

- Manufacturer's name

- Thinning instructions

- Application Instructions

- Color name and number

B. Store materials not in actual use in tightly covered containers. Maintain containers used in storage of paint in a clean condition, free of foreign materials and residue.

Protect from freezing where necessary. Keep storage area neat and orderly. Remove oily rags and waste daily. Take all precautions to ensure that 
workmen and work areas are adequately protected from fire hazards and health hazards resulting from handling, mixing and application of paints.

\section{PART 2 - PRODUCTS}

2.01 MATERIALS - The federal specifications listed below establish minimum acceptable quality for paint materials. Provide written certification from paint manufacturer that materials provided meet or exceed these minimums.

TT-P-19 Paint, acrylic emulsion, exterior.

TT-P-25 Primer coating, exterior, undercoat for wood, ready-mixed, white and tints.

TT-P-29 Paint, latex base, interior, flat, white and tints.

TT-P-86 Paint, red-lead base, ready mixed.

TT-V-86 Varnish, oil, rubbing (satin finish).

TT-E-489 Enamel, alkyd, gloss, for exterior and interior surfaces, Type I (bulk), Class A (air-drying).

TT-E-509 Enamel, alkyd, semi-gloss, interior, odorless, white or tints.

TT-E-543 Enamel, undercoat, interior, tints and white.

TT-P-636 Primer coating, alkyd, wood and ferrous metal.

TT-P-641 Primer coating, zinc dust-zinc oxide.

TT-P-650 Primer coating, latex base, interior, white.

TT-P-1511 Paint, latex, interior, tints and white, Type I (Semigloss).

\subsection{MISCELLANEOUS MATERIALS}

A. Fire-retardant paint shall be a coating that is U.L. listed as having a flamespread and smoke-developed rating of 25 or less. Primer for fire-retardant paint shall be one that is recommended by the manufacturer of the paint.

B. Fungicide Agent shall be of a type that will not adversely affect the color or durability of the paint. The fungicide agent shall be added to the paint at the factory or mixed in the proportions recommended by the manufacturer. The amount of fungicide agent added shall be at least enough to render the fabric mildew-proof.

C. Block filler shall be a vinyl acrylic latex based filler coating that is acceptable for both interior and exterior use.

D. Thinner shall be a type that is recommended by the manufacturer of the paint, and shall be used in accordance with the manufacturer's instructions. Use odorless thinner with odorless enamel (TT-E-509). 
2.03 COLORS - Paint colors, if not indicated in the contract document, shall be as selected from paint manufacturer's color chips. Match existing colors where directed.

\section{PART 3 - EXECUTION}

\subsection{SURFACE PREPARATION}

A. General: Perform preparation and cleaning procedures in accordance with paint manufacturer's instructions and as herein specified, for each particular substrate condition.

Do not paint over dirt, rust, scale, grease, moisture, scuffed surfaces, or conditions otherwise detrimental to formation of a durable paint film.

B. Cementitious Materials: Prepare cementitious surfaces of concrete and concrete block, to be painted by removing efflorescence, chalk, dust, dirt, grease, oils, and by roughening as required to remove glaze.

C. Wood: Clean wood surfaces to be painted of dirt, oil, or other foreign substances with scrapers, mineral spirits, and sandpaper, as required. Sandpaper smooth those finished surfaces exposed to view, and dust off. After priming, fill holes and imperfections in finish surfaces with putty or plastic wood-filler. Sandpaper smooth when dried.

D. Ferrous Metals: Clean ferrous surfaces, which are not galvanized or shopcoated, of oil, grease, dirt, loose mill scale and other foreign substances by solvent or mechanical cleaning. abraded or corroded spots on shop-coated surfaces shall be wire brushed and touched up with the same paint as the shop coạt.

E. Galvanized Surfaces: Clean free of oil and surface contaminants with nonpetroleum-based solvent.

F. Gypsum Board and Plaster Surface: Repair blemishes, irregularities, and damaged areas.

\subsection{APPLICATION}

A. General: Apply paint in accordance with manufacturer's directions. Use applicators and techniques best suited for substrate and type of materiel being applied. Spray method shall be used only when so specified by notes on drawings or special specifications for the project. 
B. Atmospheric Conditions: Apply water-base paint only when temperature of surfaces to be painted and surrounding air temperatures are between $50^{\circ} \mathrm{F}$ $\left(10^{\circ} \mathrm{C}\right)$ and $90^{\circ} \mathrm{F}\left(32^{\circ} \mathrm{C}\right)$, unless otherwise permitted by the paint manufacturer's printed instructions.

Apply solvent-thinned paint only when temperature of surfaces to be painted and surrounding air temperatures are between $45^{\circ} \mathrm{F}\left(7^{\circ} \mathrm{C}\right)$ and $95^{\circ} \mathrm{F}\left(35^{\circ} \mathrm{C}\right)$, unless otherwise permitted by the paint manufacture's printed instructions.

Do not apply paint in snow, rain, fog, or mist, or when relative humidity exceeds $85 \%$, or to damp or wet surfaces, unless otherwise permitted by paint manufacturer's printed instructions.

Painting may be continued during inclement weather if areas and surfaces to be painted are enclosed and heated within temperature limits specified by the paint manufacture during application and drying periods.

C. Rate of Application: The contractor shall assume the responsibility that the number of coats specified in the painting schedule will provide "full coverage " of underlying surfaces, and "full coverage" shall be acceptable. If, in the judgment of the Sandia Delegated Representative, "full coverage" is not accomplished in the number of coats specified, the contractor shall apply additional coats at no additional cost until "full coverage" accomplished. finish paints shall be applied without leaving drops sags, ridges, waves, voids, holidays, and defective brushing.

D. Color Coding of Sprinkler systems: Paint all exterior control valve indicator posts, water motor alarm and gongs, fire department connections, and sprinkler system trains through walls afire Protection Red.

Also, paint interior sprinkler system riser to ceiling line or to the first horizontal run, alarm valve, and all other related accessories "Fire Protection Red."

\subsection{CLEAN-UP AND PROTECTION}

Protect work of other trades, whether to be painted or not, against damage by painting and finishing work. Correct any damage by cleaning, repairing or replacing, and repainting, as acceptable to the Sandia Delegated Representative.

Upon completion of painting work, clean window glass and other paint-spattered surfaces. Remove spattered paint by proper methods of washing and scraping, using care not to scratch or otherwise damage finished surfaces.

\subsection{PAINTING SCHEDULE}


$\underline{\text { Surface }}$

A. Wood (painted)

1. New

a.Interior

b. Exterior
Primer

First Coat

Finish Coat

2. Previously Painted
a. Interior
b. Exterior
Fire Retardant Fire Retardant
TT-E-489
TT-E-489

Fire Retardant

Fire Retardant Fire Retardant

TT-P-25

TT-E-489

TT-E-489

B. Wood (varnished)

TT-V-86

TT-V-86

C. Ferrous Metal

1. New
a. Interior
TT-P-636 or
TT-E-543
TT-E-509
b. Exterior TT-P-636 or
TT-E-489
TT-E-489
TT-P-86

2. Previously Painted
a. Interior
TT-E-543
TT-E-509
b. Exterior
TT-E-489
TT-E-489

D. Galvanized Metal

1. New
a. Interior TT-P 641
TT-E-543
TT-E-509
b. Exterior TT-P-641
TT-E-489
TT-E-489

2. Previously Painted
a. Interior
TT-E-509
TT-E-509
b. Exterior
TT-E-489
TT-E-489

E. Concrete Block

1. New
a. Interior Block Filler
TT-E-543 or
TT-E-509 or
TT-P-1511
TT-P-1511
b. Exterior Block Filler
TT-P-19
TT-P-19

2. Previously Painted 

a. Interior -
TT-E-543 or
TT-E-509 or
b. Exterior
TT-P-1511
TT-P-1511
TT-P-19
TT-P-19

F. Concrete and Stucco

1. New
a. Interior
TT-P-1511
TT-P-1511
b. Exterior
TT-P-19
TT-P-19

2. Previously Painted
a. Interior
TT-P-1511
TT-P-1511
b. Exterior
$-$
TT-P-19
TT-P-19

G. Gypsum Wallboard and Plaster

1. New TT-P-650

2. Previously Painted

TT-P-1511 TT-P-1511

TT-P-1511 TT-P-1511

H. Canvas-Covered

TT-P-29

TT-P-29

insulation on pipe

w/ fungicidal and ductwork

agent

End Of Section 09900 


\section{ATTACHMENT $F$}

Volumetric Moisture Content Calculations 
Table F1: Moisture Content for Phase 1 Probe, 138' Cable, 6\% Bentonite

\begin{tabular}{|c|c|c|c|c|c|c|}
\hline M of Wet Soil & M of Solids & $\begin{array}{c}\text { Moisture Content } \\
(\mathrm{w})\end{array}$ & $\begin{array}{c}\text { Total Mass } \\
(\mathrm{lbs})\end{array}$ & $\begin{array}{c}\text { Total Mass } \\
(\mathrm{g})\end{array}$ & V of Water & Volumetric w \\
\hline 125.2 & 122.4 & $2.29 \%$ & 15.25 & 6713.24 & 150.14 & $3.70 \%$ \\
\hline 209 & 199 & $5.03 \%$ & 15.3 & 6735.92 & 322.29 & $7.94 \%$ \\
\hline 125.1 & 116.6 & $7.29 \%$ & 16.81 & 7420.85 & 504.21 & $12.42 \%$ \\
\hline 80 & 71.9 & $11.27 \%$ & 18.35 & 8119.39 & 822.09 & $20.26 \%$ \\
\hline 198.3 & 173.5 & $14.29 \%$ & 18.8 & 8323.51 & 1040.96 & $25.65 \%$ \\
\hline
\end{tabular}

Table F2: Moisture Content for Phase 1 Probe, 59' Cable, 6\% Bentonite

\begin{tabular}{|c|c|c|c|c|c|c|}
\hline M of Wet Soil & M of Solids & $\begin{array}{c}\text { Moisture Content } \\
(\mathrm{w})\end{array}$ & $\begin{array}{c}\text { Total Mass } \\
(\mathrm{lbs})\end{array}$ & $\begin{array}{c}\text { Total Mass } \\
(\mathrm{g})\end{array}$ & V of Water & $\begin{array}{c}\text { Volumetric } \\
\mathrm{w}\end{array}$ \\
\hline 157.4 & 150.8 & $4.38 \%$ & 15.08 & 6636.124467 & 278.26 & $6.86 \%$ \\
\hline 95.4 & 89 & $7.19 \%$ & 16.38 & 7225.800599 & 484.75 & $11.94 \%$ \\
\hline 155.2 & 140.8 & $10.23 \%$ & 16.88 & 7452.599111 & 691.48 & $17.04 \%$ \\
\hline 126.5 & 110.7 & $14.27 \%$ & 17.94 & 7933.411957 & 990.89 & $24.42 \%$ \\
\hline 209.4 & 179.3 & $16.79 \%$ & 18.59 & 8228.250023 & 1182.76 & $29.14 \%$ \\
\hline
\end{tabular}

Table F3: Moisture Content for Phase 1 Probe, 82' Cable, 6\% Bentonite

\begin{tabular}{|c|c|c|c|c|c|c|}
\hline M of Wet Soil & M of Solids & $\begin{array}{c}\text { Moisture Content } \\
(\mathrm{w})\end{array}$ & $\begin{array}{c}\text { Total Mass } \\
(\mathrm{lbs})\end{array}$ & $\begin{array}{c}\text { Total Mass } \\
(\mathrm{g})\end{array}$ & V of Water & $\begin{array}{c}\text { Volumetric } \\
\mathrm{w}\end{array}$ \\
\hline 157.4 & 150.8 & $4.38 \%$ & 15.08 & 6636.12 & 278.26 & $6.86 \%$ \\
\hline 95.4 & 89 & $7.19 \%$ & 16.38 & 7225.80 & 484.75 & $11.94 \%$ \\
\hline 155.2 & 140.8 & $10.23 \%$ & 16.88 & 7452.60 & 691.48 & $17.04 \%$ \\
\hline 126.5 & 110.7 & $14.27 \%$ & 17.94 & 7933.41 & 990.89 & $24.42 \%$ \\
\hline 209.4 & 179.3 & $16.79 \%$ & 18.59 & 8228.25 & 1182.76 & $29.14 \%$ \\
\hline
\end{tabular}

Table F4: Moisture Content for Phase 1 Probe, 98' Cable, 6\% Bentonite

\begin{tabular}{|c|c|c|c|c|c|c|}
\hline M of Wet Soil & M of Solids & $\begin{array}{c}\text { Moisture Content } \\
(\mathrm{w})\end{array}$ & $\begin{array}{c}\text { Total Mass } \\
(\mathrm{lbs})\end{array}$ & $\begin{array}{c}\text { Total Mass } \\
(\mathrm{g})\end{array}$ & Vof Water & $\begin{array}{c}\text { Volumetric } \\
\mathrm{w}\end{array}$ \\
\hline 197.7 & 190.4 & $3.83 \%$ & 15.52 & 6835.71 & 252.41 & $6.22 \%$ \\
\hline 92.7 & 86.9 & $6.67 \%$ & 16.15 & 7121.47 & 445.57 & $10.98 \%$ \\
\hline 122.9 & 112.8 & $8.95 \%$ & 16.93 & 7475.28 & 614.32 & $15.14 \%$ \\
\hline 132.6 & 116.9 & $13.43 \%$ & 17.75 & 7847.23 & 929.12 & $22.89 \%$ \\
\hline 189.8 & 163.6 & $16.01 \%$ & 19.01 & 8418.76 & 1162.13 & $28.64 \%$ \\
\hline
\end{tabular}


Table F5: Moisture Content for Phase 1 Probe, 120' Cable, 6\% Bentonite

\begin{tabular}{|c|c|c|c|c|c|c|}
\hline M of Wet Soil & M of Solids & $\begin{array}{c}\text { Moisture Content } \\
(\mathrm{w})\end{array}$ & $\begin{array}{c}\text { Total Mass } \\
(\mathrm{lbs})\end{array}$ & $\begin{array}{c}\text { Total Mass } \\
(\mathrm{g})\end{array}$ & $\begin{array}{c}\text { V of Water } \\
\text { Volumetric } \\
\mathrm{w}\end{array}$ \\
\hline 127.4 & 122.6 & $3.92 \%$ & 15.61 & 6876.53 & 259.08 & $6.38 \%$ \\
\hline 108.6 & 102 & $6.47 \%$ & 15.65 & 6894.67 & 419.01 & $10.32 \%$ \\
\hline 107.4 & 98 & $9.59 \%$ & 16.4 & 7234.87 & 633.22 & $15.60 \%$ \\
\hline 125 & 110.8 & $12.82 \%$ & 17.29 & 7638.57 & 867.74 & $21.38 \%$ \\
\hline 191.4 & 164.9 & $16.07 \%$ & 18.1 & 8005.99 & 1108.46 & $27.31 \%$ \\
\hline
\end{tabular}

Table F6: Moisture Content for Phase 1 Probe, 59' Cable, Compacted Native Soil

\begin{tabular}{|c|c|c|c|c|c|c|}
\hline M of Wet Soil & M of Solids & $\begin{array}{c}\text { Moisture Content } \\
(\mathrm{w})\end{array}$ & $\begin{array}{c}\text { Total Mass } \\
(\mathrm{lbs})\end{array}$ & $\begin{array}{c}\text { Total Mass } \\
(\mathrm{g})\end{array}$ & $\begin{array}{c}\text { V of Water } \\
\text { (Volumetric } \\
\mathrm{w}\end{array}$ \\
\hline 145.7 & 143.7 & $1.39 \%$ & 14.5 & 6577.16 & 90.28 & $2.22 \%$ \\
\hline 70.7 & 67.4 & $4.90 \%$ & 15.6 & 6871.99 & 320.76 & $7.90 \%$ \\
\hline 110.1 & 102.3 & $7.62 \%$ & 17.22 & 7606.82 & 538.90 & $13.28 \%$ \\
\hline 117.4 & 106.3 & $10.44 \%$ & 18.56 & 8214.64 & 776.68 & $19.14 \%$ \\
\hline 231.6 & 200.2 & $15.68 \%$ & 18.3 & 8096.71 & 1097.74 & $27.05 \%$ \\
\hline
\end{tabular}

Table F7: Moisture Content for Phase 1 Probe, 82' Cable, Compacted Native Soil

\begin{tabular}{|c|c|c|c|c|c|c|}
\hline M of Wet Soil & M of Solids & $\begin{array}{c}\text { Moisture Content } \\
(\mathrm{w})\end{array}$ & $\begin{array}{c}\text { Total Mass } \\
(\mathrm{lbs})\end{array}$ & $\begin{array}{c}\text { Total Mass } \\
(\mathrm{g})\end{array}$ & $\begin{array}{c}\text { V of Water } \\
\text { Volumetric } \\
\mathrm{w}\end{array}$ \\
\hline 145.7 & 143.7 & $1.39 \%$ & 14.5 & 6577.16 & 90.28 & $2.22 \%$ \\
\hline 70.7 & 67.4 & $4.90 \%$ & 15.6 & 6871.99 & 320.76 & $7.90 \%$ \\
\hline 110.1 & 102.3 & $7.62 \%$ & 17.22 & 7606.82 & 538.90 & $13.28 \%$ \\
\hline 117.4 & 106.3 & $10.44 \%$ & 18.56 & 8214.64 & 776.68 & $19.14 \%$ \\
\hline 231.6 & 200.2 & $15.68 \%$ & 18.3 & 8096.71 & 1097.74 & $27.05 \%$ \\
\hline
\end{tabular}

Table F8: Moisture Content for Phase 1 Probe, 98' Cable, Compacted Native Soil

\begin{tabular}{|c|c|c|c|c|c|c|}
\hline M of Wet Soil & M of Solids & $\begin{array}{c}\text { Moisture Content } \\
(\mathrm{w})\end{array}$ & $\begin{array}{c}\text { Total Mass } \\
(\mathrm{lbs})\end{array}$ & $\begin{array}{c}\text { Total Mass } \\
(\mathrm{g})\end{array}$ & V of Water & $\begin{array}{c}\text { Volumetric } \\
\mathrm{w}\end{array}$ \\
\hline 139.5 & 135 & $3.33 \%$ & 14.31 & 6490.97 & 209.39 & $5.16 \%$ \\
\hline 95 & 89.2 & $6.50 \%$ & 15.85 & 6985.39 & 426.48 & $10.51 \%$ \\
\hline 126.9 & 115.2 & $10.16 \%$ & 17.74 & 7842.69 & 723.09 & $17.82 \%$ \\
\hline 212.8 & 191.8 & $10.95 \%$ & 18.43 & 8155.67 & 804.84 & $19.83 \%$ \\
\hline 170 & 146.6 & $15.96 \%$ & 18.27 & 8083.10 & 1112.61 & $27.42 \%$ \\
\hline
\end{tabular}


Table F9: Moisture Content for Phase 1 Probe, 120' Cable, Compacted Native Soil

\begin{tabular}{|c|c|c|c|c|c|c|}
\hline M of Wet Soil & M of Solids & $\begin{array}{c}\text { Moisture Content } \\
(\mathrm{w})\end{array}$ & $\begin{array}{c}\text { Total Mass } \\
(\mathrm{lbs})\end{array}$ & $\begin{array}{c}\text { Total Mass } \\
(\mathrm{g})\end{array}$ & V of Water & $\begin{array}{c}\text { Volumetric } \\
\mathrm{w}\end{array}$ \\
\hline 160.7 & 157.7 & $1.90 \%$ & 14.6 & 6622.52 & 123.63 & $3.05 \%$ \\
\hline 86 & 82 & $4.88 \%$ & 14.93 & 6568.08 & 305.49 & $7.53 \%$ \\
\hline 130.1 & 120.9 & $7.61 \%$ & 16.68 & 7361.88 & 520.59 & $12.83 \%$ \\
\hline 152.2 & 136.5 & $11.50 \%$ & 18.26 & 8078.56 & 833.33 & $20.53 \%$ \\
\hline 217.5 & 188.6 & $15.32 \%$ & 18.16 & 8033.20 & 1067.40 & $26.30 \%$ \\
\hline
\end{tabular}

Table F10: Moisture Content for Phase 1 Probe, 138' Cable, Compacted Native Soil

\begin{tabular}{|c|c|c|c|c|c|c|}
\hline M of Wet Soil & M of Solids & $\begin{array}{c}\text { Moisture Content } \\
(\mathrm{w})\end{array}$ & $\begin{array}{c}\text { Total Mass } \\
(\mathrm{lbs})\end{array}$ & $\begin{array}{c}\text { Total Mass } \\
(\mathrm{g})\end{array}$ & V of Water & $\begin{array}{c}\text { Volumetric } \\
\mathrm{w}\end{array}$ \\
\hline 189.2 & 185.8 & $1.83 \%$ & 14.66 & 6649.73 & 119.50 & $2.94 \%$ \\
\hline 84.5 & 80.4 & $5.10 \%$ & 14.99 & 6595.30 & 320.01 & $7.89 \%$ \\
\hline 143.7 & 133 & $8.05 \%$ & 16.66 & 7352.81 & 547.50 & $13.49 \%$ \\
\hline 149.8 & 134.3 & $11.54 \%$ & 18.34 & 8114.85 & 839.65 & $20.69 \%$ \\
\hline 213.8 & 185.2 & $15.44 \%$ & 18.49 & 8182.89 & 1094.62 & $26.97 \%$ \\
\hline
\end{tabular}

Table F11: Moisture Content for Phase 1 Probe, 59' Cable, Sand

\begin{tabular}{|c|c|c|c|c|c|c|}
\hline M of Wet Soil & M of Solids & $\begin{array}{c}\text { Moisture Content } \\
(\mathrm{w})\end{array}$ & $\begin{array}{c}\text { Total Mass } \\
(\mathrm{lbs})\end{array}$ & $\begin{array}{c}\text { Total Mass } \\
(\mathrm{g})\end{array}$ & V of Water & $\begin{array}{c}\text { Volumetric } \\
\mathrm{w}\end{array}$ \\
\hline 183.7 & 181.9 & $0.99 \%$ & 15.28 & 6726.84 & 65.91 & $1.62 \%$ \\
\hline 204.5 & 197.7 & $3.44 \%$ & 15 & 6599.84 & 219.46 & $5.41 \%$ \\
\hline 194.8 & 183.3 & $6.27 \%$ & 14.73 & 6477.37 & 382.39 & $9.42 \%$ \\
\hline 271.7 & 244.3 & $11.22 \%$ & 15.99 & 7048.90 & 710.86 & $17.52 \%$ \\
\hline 297.8 & 249.9 & $19.17 \%$ & 17.09 & 7547.85 & 1214.04 & $29.91 \%$ \\
\hline
\end{tabular}

Table F12: Moisture Content for Phase 1 Probe, 82' Cable, Sand

\begin{tabular}{|c|c|c|c|c|c|c|}
\hline M of Wet Soil & M of Solids & $\begin{array}{c}\text { Moisture Content } \\
(\mathrm{w})\end{array}$ & $\begin{array}{c}\text { Total Mass } \\
(\mathrm{lbs})\end{array}$ & $\begin{array}{c}\text { Total Mass } \\
(\mathrm{g})\end{array}$ & V of Water & $\begin{array}{c}\text { Volumetric } \\
\mathrm{w}\end{array}$ \\
\hline 183.7 & 181.9 & $0.99 \%$ & 15.28 & 6726.84 & 65.91 & $1.62 \%$ \\
\hline 204.5 & 197.7 & $3.44 \%$ & 15 & 6599.84 & 219.46 & $5.41 \%$ \\
\hline 194.8 & 183.3 & $6.27 \%$ & 14.73 & 6477.37 & 382.39 & $9.42 \%$ \\
\hline 271.7 & 244.3 & $11.22 \%$ & 15.99 & 7048.90 & 710.86 & $17.52 \%$ \\
\hline 297.8 & 249.9 & $19.17 \%$ & 17.09 & 7547.85 & 1214.04 & $29.91 \%$ \\
\hline
\end{tabular}


Table F13: Moisture Content for Phase 1 Probe, 138' Cable, Sand

\begin{tabular}{|c|c|c|c|c|c|c|}
\hline M of Wet Soil & M of Solids & $\begin{array}{c}\text { Moisture Content } \\
(\mathrm{w})\end{array}$ & $\begin{array}{c}\text { Total Mass } \\
(\mathrm{lbs})\end{array}$ & $\begin{array}{c}\text { Total Mass } \\
(\mathrm{g})\end{array}$ & V of Water & $\begin{array}{c}\text { Volumetric } \\
\mathrm{w}\end{array}$ \\
\hline 161.8 & 159.9 & $1.19 \%$ & 15.47 & 6813.03 & 80.00 & $1.97 \%$ \\
\hline 141.1 & 136.7 & $3.22 \%$ & 14.5 & 6373.04 & 198.73 & $4.90 \%$ \\
\hline 223.1 & 209.9 & $6.29 \%$ & 15.33 & 6749.52 & 399.34 & $9.84 \%$ \\
\hline 192.4 & 175.8 & $9.44 \%$ & 15.35 & 6758.60 & 583.12 & $14.37 \%$ \\
\hline 284.4 & 253.7 & $12.10 \%$ & 15.89 & 7003.54 & 756.01 & $18.63 \%$ \\
\hline 279.7 & 234 & $19.53 \%$ & 16.8 & 7416.31 & 1211.75 & $29.86 \%$ \\
\hline
\end{tabular}

Table F14: Moisture Content for Phase 1 Probe, 59' Cable, Uncompacted Native Soil

\begin{tabular}{|c|c|c|c|c|c|c|}
\hline M of Wet Soil & M of Solids & $\begin{array}{c}\text { Moisture Content } \\
(\mathrm{w})\end{array}$ & $\begin{array}{c}\text { Total Mass } \\
(\mathrm{lbs})\end{array}$ & $\begin{array}{c}\text { Total Mass } \\
(\mathrm{g})\end{array}$ & V of Water & $\begin{array}{c}\text { Volumetric } \\
\mathrm{w}\end{array}$ \\
\hline 136.8 & 134.8 & $1.48 \%$ & 13.68 & 6205.21 & 90.72 & $2.24 \%$ \\
\hline 175.6 & 167.8 & $4.65 \%$ & 12.29 & 5574.71 & 247.62 & $6.10 \%$ \\
\hline 96 & 90.4 & $6.19 \%$ & 12.09 & 5483.99 & 319.90 & $7.88 \%$ \\
\hline 118.2 & 108.7 & $8.74 \%$ & 14.83 & 6726.84 & 540.65 & $13.32 \%$ \\
\hline 178.9 & 160.1 & $11.74 \%$ & 15.68 & 7112.40 & 747.42 & $18.42 \%$ \\
\hline 246.9 & 213.7 & $15.54 \%$ & 16.83 & 7634.04 & 1026.53 & $25.29 \%$ \\
\hline
\end{tabular}

Table F15: Moisture Content for Phase 1 Probe, 82' Cable, Uncompacted Native Soil

\begin{tabular}{|c|c|c|c|c|c|c|}
\hline M of Wet Soil & M of Solids & $\begin{array}{c}\text { Moisture Content } \\
(\mathrm{w})\end{array}$ & $\begin{array}{c}\text { Total Mass } \\
(\mathrm{lbs})\end{array}$ & $\begin{array}{c}\text { Total Mass } \\
(\mathrm{g})\end{array}$ & V of Water & $\begin{array}{c}\text { Volumetric } \\
\mathrm{w}\end{array}$ \\
\hline 136.8 & 134.8 & $1.48 \%$ & 13.68 & 6205.21 & 90.72 & $2.24 \%$ \\
\hline 175.6 & 167.8 & $4.65 \%$ & 12.29 & 5574.71 & 247.62 & $6.10 \%$ \\
\hline 96 & 90.4 & $6.19 \%$ & 12.09 & 5483.99 & 319.90 & $7.88 \%$ \\
\hline 118.2 & 108.7 & $8.74 \%$ & 14.83 & 6726.84 & 540.65 & $13.32 \%$ \\
\hline 178.9 & 160.1 & $11.74 \%$ & 15.68 & 7112.40 & 747.42 & $18.42 \%$ \\
\hline 246.9 & 213.7 & $15.54 \%$ & 16.83 & 7634.04 & 1026.53 & $25.29 \%$ \\
\hline
\end{tabular}

Table F16: Moisture Content for Phase 1 Probe, 98' Cable, Uncompacted Native Soil

\begin{tabular}{|c|c|c|c|c|c|c|}
\hline M of Wet Soil & M of Solids & $\begin{array}{c}\text { Moisture Content } \\
(\mathrm{w})\end{array}$ & $\begin{array}{c}\text { Total Mass } \\
(\mathrm{lbs})\end{array}$ & $\begin{array}{c}\text { Total Mass } \\
(\mathrm{g})\end{array}$ & V of Water & $\begin{array}{c}\text { Volumetric } \\
\mathrm{w}\end{array}$ \\
\hline 160.2 & 156.4 & $2.43 \%$ & 13.67 & 6200.67 & 147.08 & $3.62 \%$ \\
\hline 84.4 & 80 & $5.50 \%$ & 13.3 & 6032.84 & 314.51 & $7.75 \%$ \\
\hline 158.3 & 145.7 & $8.65 \%$ & 12.56 & 5697.18 & 453.47 & $11.17 \%$ \\
\hline 114.3 & 102.5 & $11.51 \%$ & 15.85 & 7189.51 & 742.22 & $18.29 \%$ \\
\hline 159.7 & 139 & $14.89 \%$ & 15.63 & 7089.72 & 918.96 & $22.64 \%$ \\
\hline
\end{tabular}


Table F17: Moisture Content for Phase 1 Probe, 120' Cable, Uncompacted Native Soil

\begin{tabular}{|c|c|c|c|c|c|c|}
\hline M of Wet Soil & M of Solids & $\begin{array}{c}\text { Moisture Content } \\
(\mathrm{w})\end{array}$ & $\begin{array}{c}\text { Total Mass } \\
(\mathrm{lbs})\end{array}$ & $\begin{array}{c}\text { Total Mass } \\
(\mathrm{g})\end{array}$ & V of Water & $\begin{array}{c}\text { Volumetric } \\
\mathrm{w}\end{array}$ \\
\hline 127.3 & 125.1 & $1.76 \%$ & 13.46 & 6105.42 & 105.51 & $2.60 \%$ \\
\hline 110.2 & 104.6 & $5.35 \%$ & 13.11 & 5946.66 & 302.19 & $7.45 \%$ \\
\hline 126 & 115.1 & $9.47 \%$ & 13.29 & 6028.30 & 521.50 & $12.85 \%$ \\
\hline 209.8 & 186.4 & $12.55 \%$ & 14.94 & 6776.74 & 755.84 & $18.62 \%$ \\
\hline 208.3 & 180.1 & $15.66 \%$ & 16.51 & 7488.89 & 1013.86 & $24.98 \%$ \\
\hline
\end{tabular}

Table F18: Moisture Content for Phase 1 Probe, 138' Cable, Uncompacted Native Soil

\begin{tabular}{|c|c|c|c|c|c|c|}
\hline M of Wet Soil & M of Solids & $\begin{array}{c}\text { Moisture Content } \\
(\mathrm{w})\end{array}$ & $\begin{array}{c}\text { Total Mass } \\
(\mathrm{lbs})\end{array}$ & $\begin{array}{c}\text { Total Mass } \\
(\mathrm{g})\end{array}$ & $\begin{array}{c}\text { V of Water } \\
\text { ( })\end{array}$ & $\begin{array}{c}\text { Volumetric } \\
\mathrm{w}\end{array}$ \\
\hline 83.9 & 80.3 & $4.48 \%$ & 11.5 & 5216.37 & 223.82 & $5.52 \%$ \\
\hline 199.6 & 186.8 & $6.85 \%$ & 12.48 & 5660.89 & 363.02 & $8.95 \%$ \\
\hline 127 & 115.5 & $9.96 \%$ & 13.72 & 6223.35 & 563.53 & $13.89 \%$ \\
\hline 145.8 & 128.5 & $13.46 \%$ & 16.14 & 7321.06 & 868.68 & $21.40 \%$ \\
\hline 208.7 & 175.3 & $19.05 \%$ & 16.86 & 7647.65 & 1223.92 & $30.16 \%$ \\
\hline
\end{tabular}

Table F19: Moisture Content for Phase 2 Probe, All Cables, Compacted Native Soil

\begin{tabular}{|c|c|c|c|c|c|c|}
\hline M of Wet Soil & M of Solids & $\begin{array}{c}\text { Moisture Content } \\
(\mathrm{w})\end{array}$ & $\begin{array}{c}\text { Total Mass } \\
(\mathrm{lbs})\end{array}$ & $\begin{array}{c}\text { Total Mass } \\
(\mathrm{g})\end{array}$ & $\begin{array}{c}\text { V of Water } \\
\text { Volumetric } \\
\mathrm{w}\end{array}$ \\
\hline 170.1 & 168 & $1.25 \%$ & 78.63 & 35667 & 440.33 & $2.15 \%$ \\
\hline 379.5 & 362.6 & $4.66 \%$ & 78.57 & 35640 & 1587.13 & $7.75 \%$ \\
\hline 224.8 & 210.8 & $6.64 \%$ & 85.41 & 38743 & 2412.82 & $11.77 \%$ \\
\hline 596 & 536.3 & $11.13 \%$ & 93.48 & 42892 & 4296.40 & $20.97 \%$ \\
\hline 248.2 & 215.9 & $14.96 \%$ & 95.6 & 42823 & 5572.86 & $27.20 \%$ \\
\hline
\end{tabular}

Table F20: Moisture Content for Phase 2 Probe, All Cables, Sand

\begin{tabular}{|c|c|c|c|c|c|c|}
\hline M of Wet Soil & M of Solids & $\begin{array}{c}\text { Moisture Content } \\
(\mathrm{w})\end{array}$ & $\begin{array}{c}\text { Total Mass } \\
(\mathrm{lbs})\end{array}$ & $\begin{array}{c}\text { Total Mass } \\
(\mathrm{g})\end{array}$ & V of Water & $\begin{array}{c}\text { Volumetric } \\
\mathrm{w}\end{array}$ \\
\hline 146.3 & 144.3 & $1.39 \%$ & 78.76 & 35800 & 489.41 & $2.39 \%$ \\
\hline 229.4 & 219.3 & $4.61 \%$ & 76.15 & 34615 & 1524.03 & $7.44 \%$ \\
\hline 252.8 & 236.3 & $6.98 \%$ & 80.00 & 36365 & 2373.51 & $11.58 \%$ \\
\hline 214.7 & 194.2 & $10.56 \%$ & 84.77 & 38531 & 3679.02 & $17.95 \%$ \\
\hline 344.3 & 297.9 & $15.58 \%$ & 89.89 & 40858 & 5506.28 & $26.87 \%$ \\
\hline
\end{tabular}


Table F21: Moisture Content for Phase 2 Probe, All Cables, Uncompacted Native Soil

\begin{tabular}{|c|c|c|c|c|c|c|}
\hline M of Wet Soil & M of Solids & $\begin{array}{c}\text { Moisture Content } \\
(\mathrm{w})\end{array}$ & $\begin{array}{c}\text { Total Mass } \\
(\mathrm{lbs})\end{array}$ & $\begin{array}{c}\text { Total Mass } \\
(\mathrm{g})\end{array}$ & V of Water & $\begin{array}{c}\text { Volumetric } \\
\mathrm{w}\end{array}$ \\
\hline 136.4 & 134.7 & $1.26 \%$ & 74.74 & 33971 & 423.39 & $2.07 \%$ \\
\hline 148.7 & 142.3 & $4.50 \%$ & 71.40 & 32455 & 1396.85 & $6.82 \%$ \\
\hline 218.3 & 204.4 & $6.80 \%$ & 76.51 & 34775 & 2214.26 & $10.81 \%$ \\
\hline 189 & 171.5 & $10.20 \%$ & 85.79 & 38997 & 3610.83 & $17.62 \%$ \\
\hline 256.9 & 222 & $15.72 \%$ & 92.04 & 41837 & 5683.58 & $27.74 \%$ \\
\hline
\end{tabular}




\section{ATTACHMENT G}

Plots of Third Order Polynomials for Individual Cable Lengths 
$6 \%$ Bentonite, Phase I, $59^{`}$ Cable

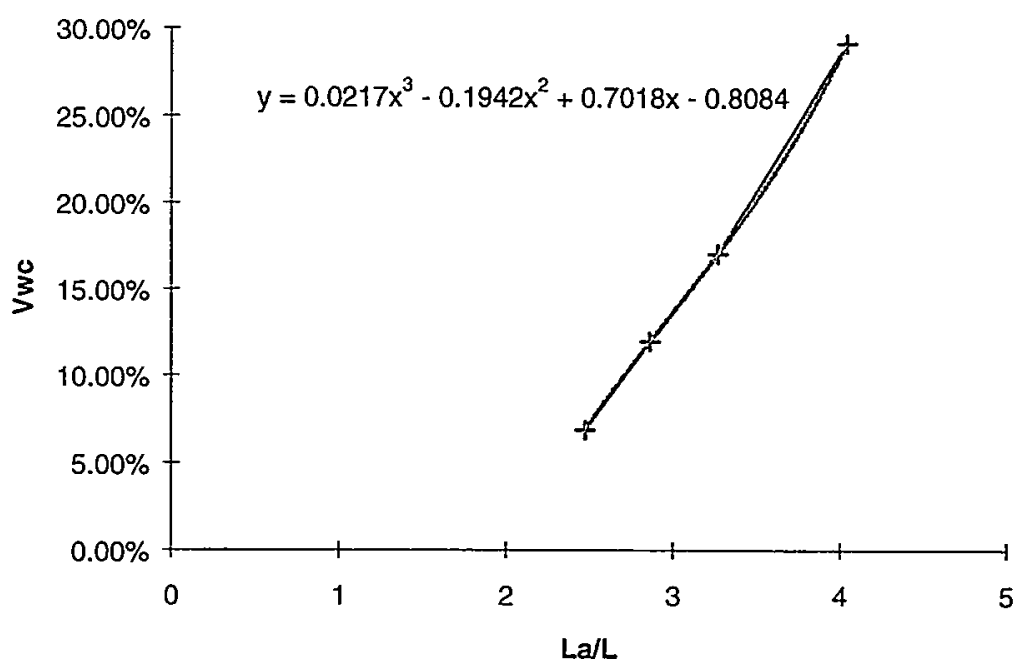

Figure G-1

6\% Bentonite, Phase 1, $82^{\prime}$ Cable

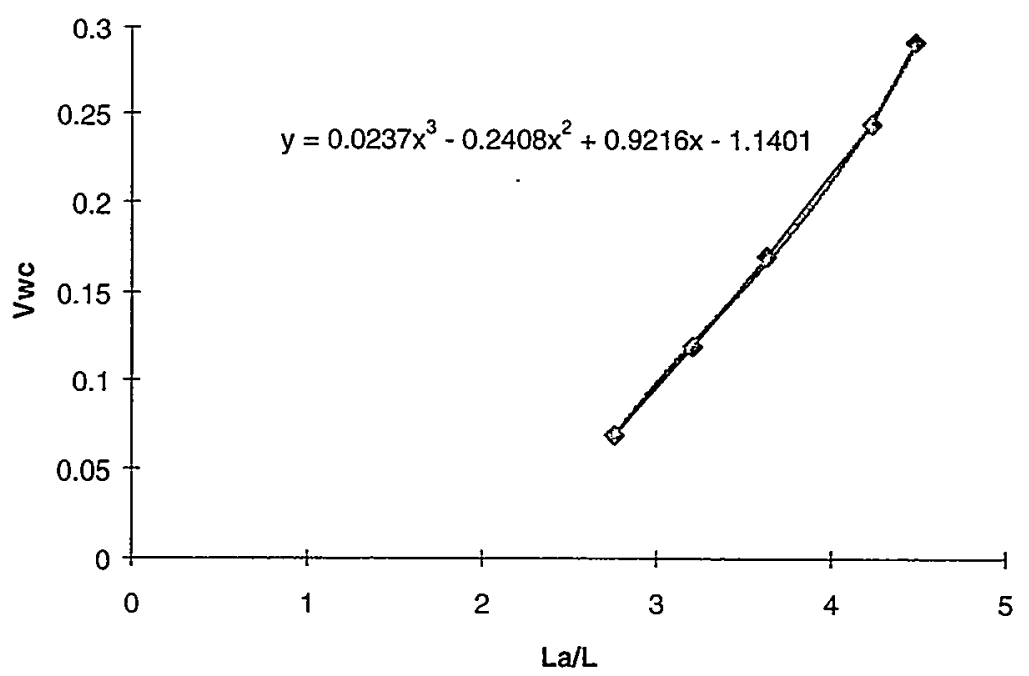

Figure G-2 
$6 \%$ Bentonite, Phase I, 98 ' Cable

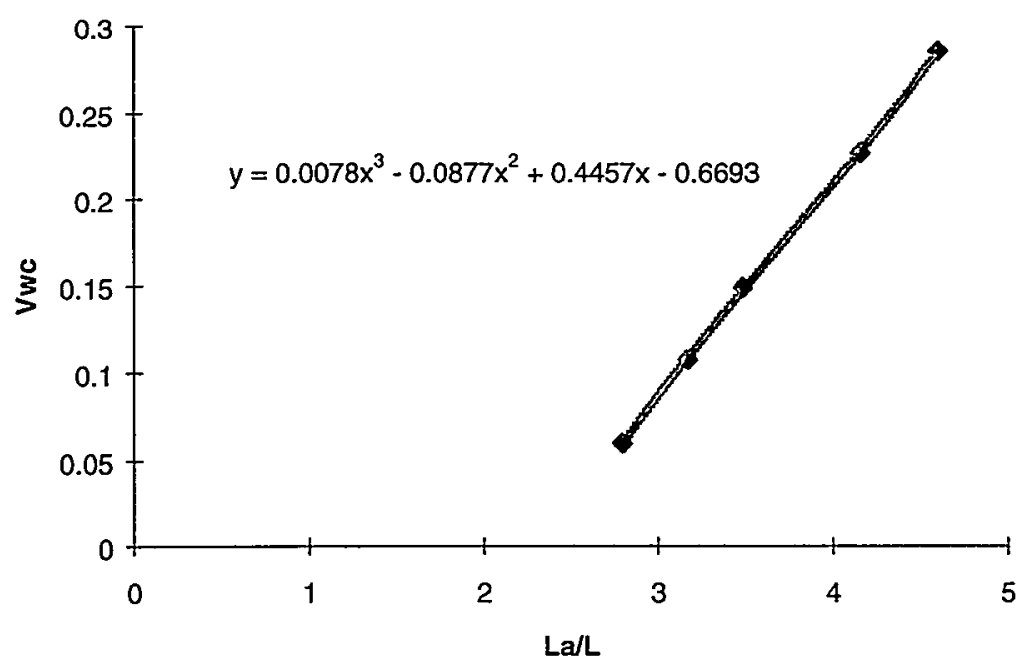

Figure G-3

$6 \%$ Bentonite, Phase I, $120^{2}$ Cable

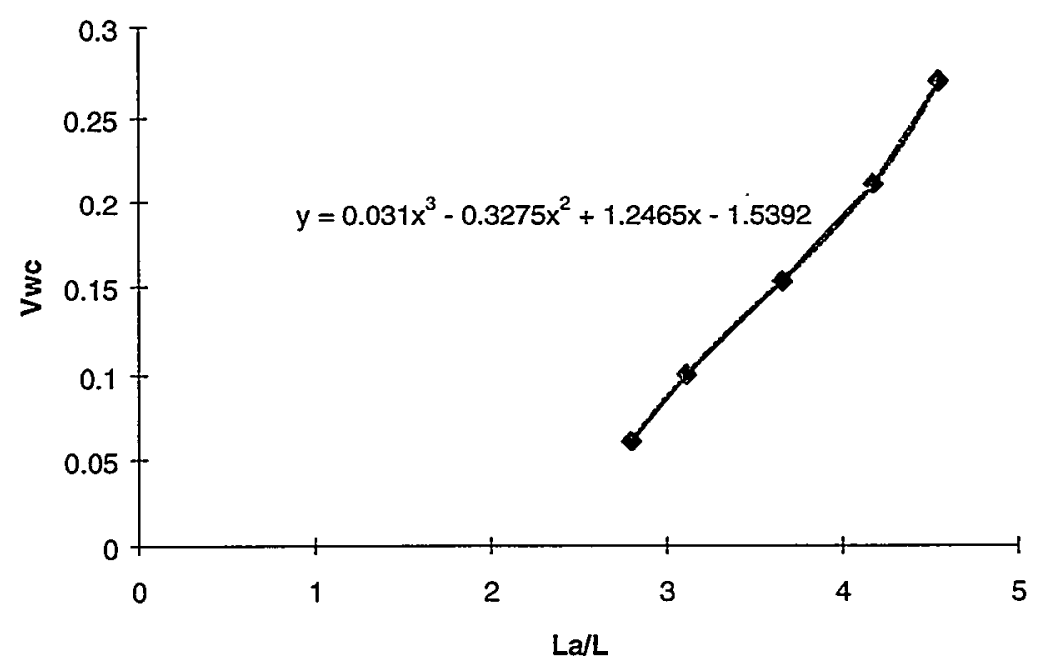

Figure G-4 
6\% Bentonite, Phase I, 138' Cable

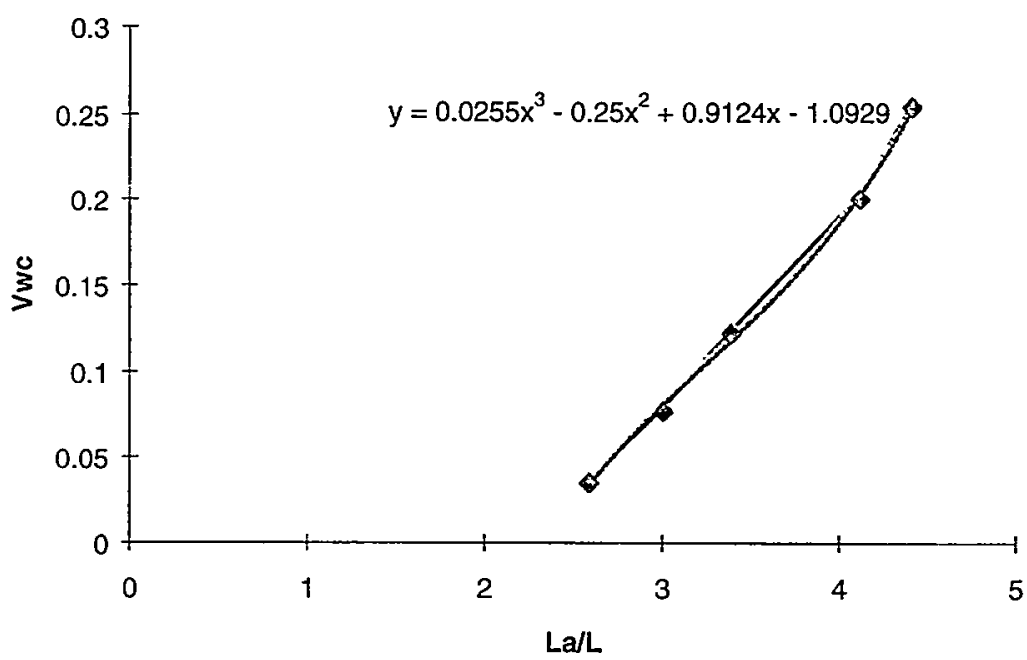

Figure G-5

\section{Compacted Native Soil, Phase I, 59' Cable}

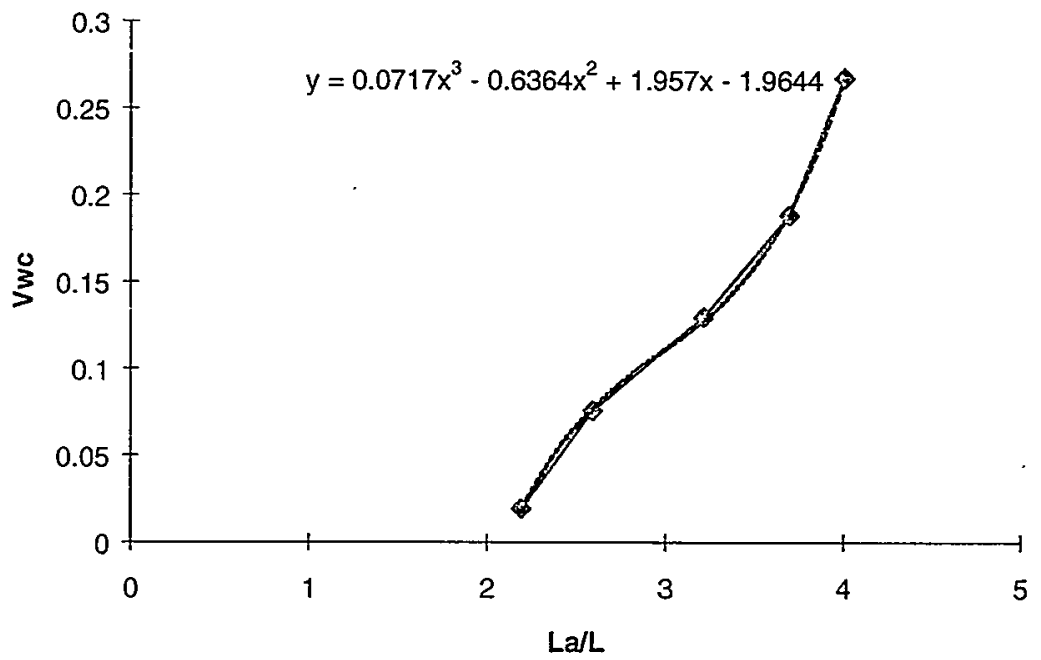

Figure G-6 
Compacted Native Soil, Phase I, 82' Cable

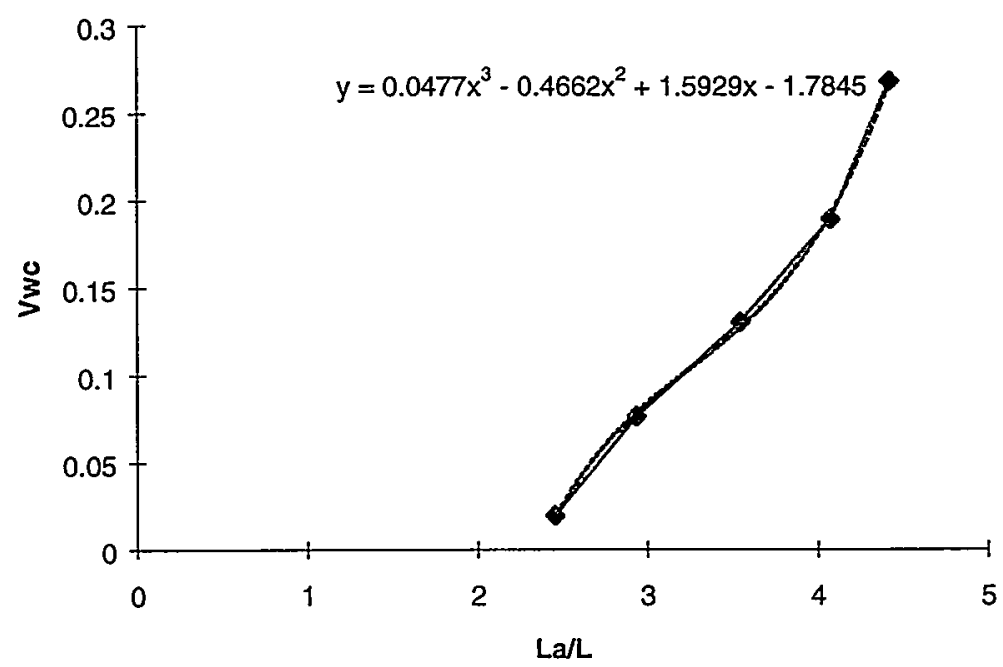

Figure G-7

Compacted Native Soil, Phase I, 98' Cable

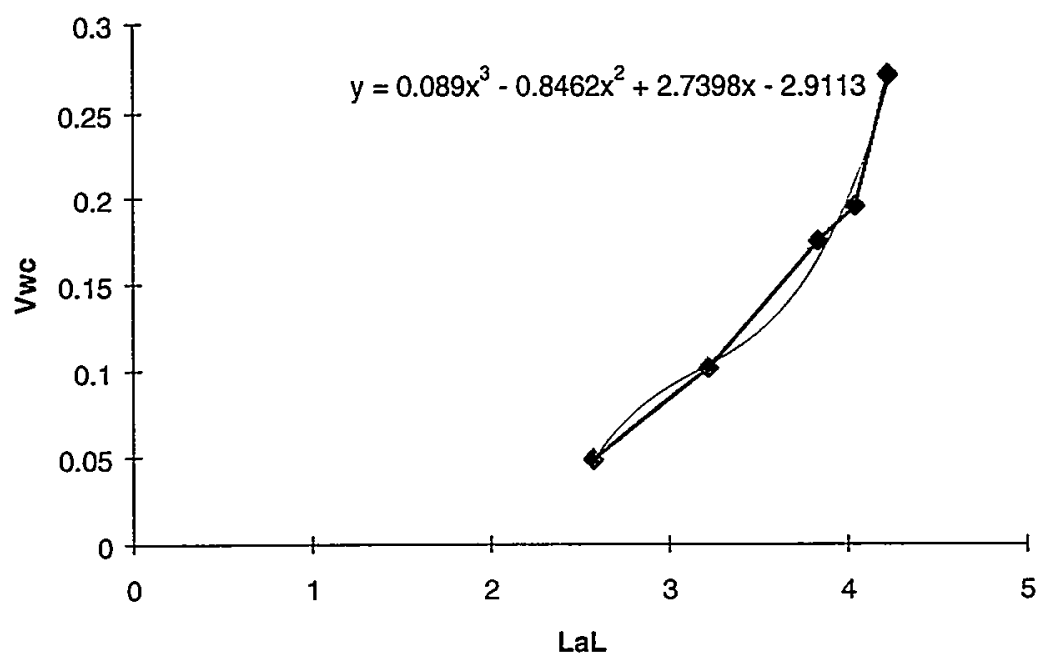

Figure G-8 


\section{Compacted Native Soil, Phase I, $120^{1}$ Cable}

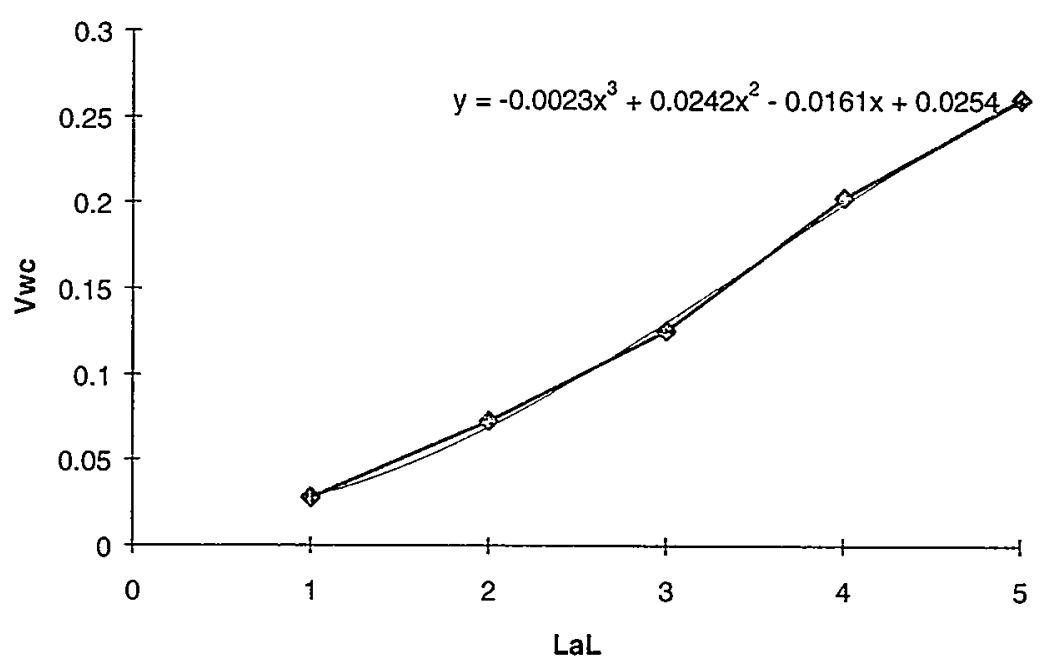

Figure G-9

Compacted Native Soil, Phase I, 138' Cable

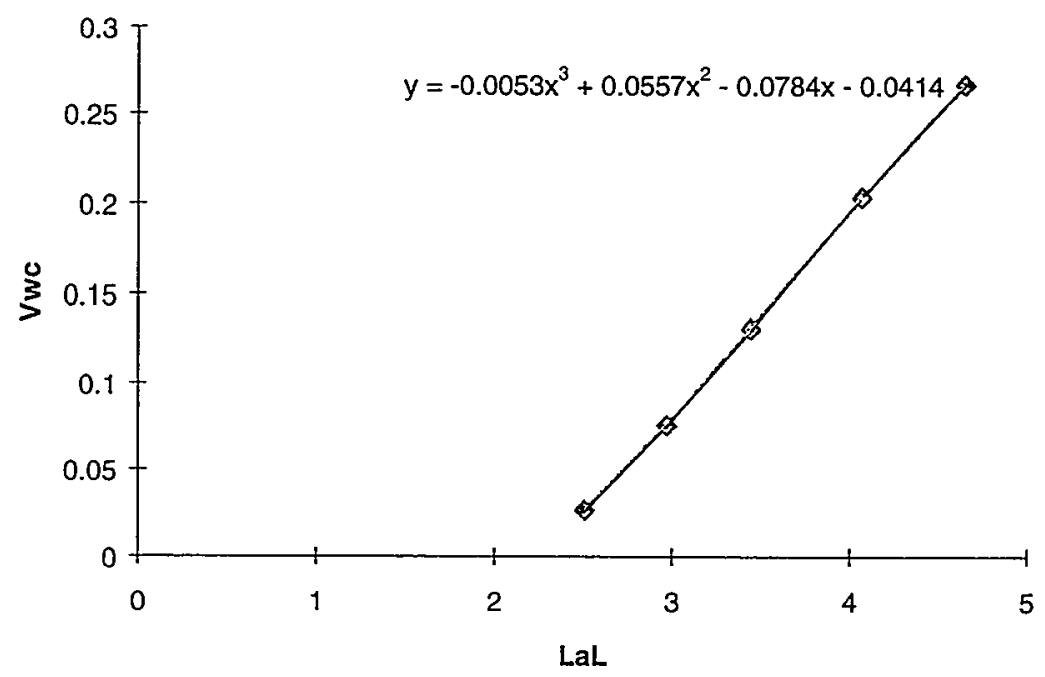

Figure G-10 
Uncompacted Native Soil, Phase I, 59' Cable

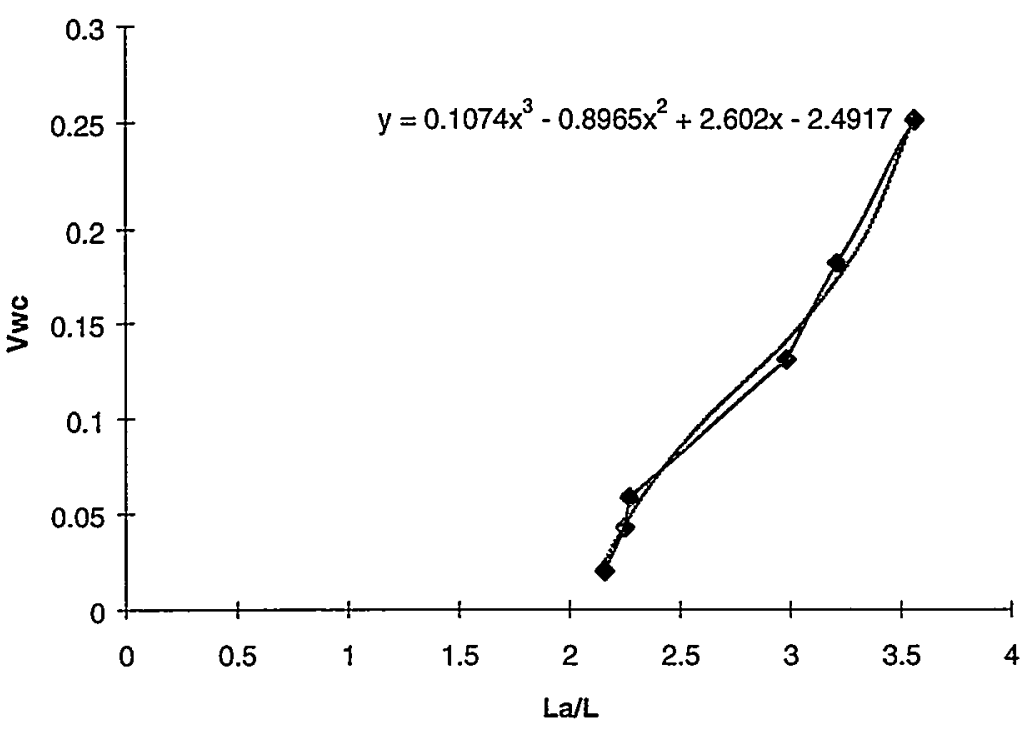

Figure G-11

\section{Uncompacted Native Soil, Phase I, 82' Cable}

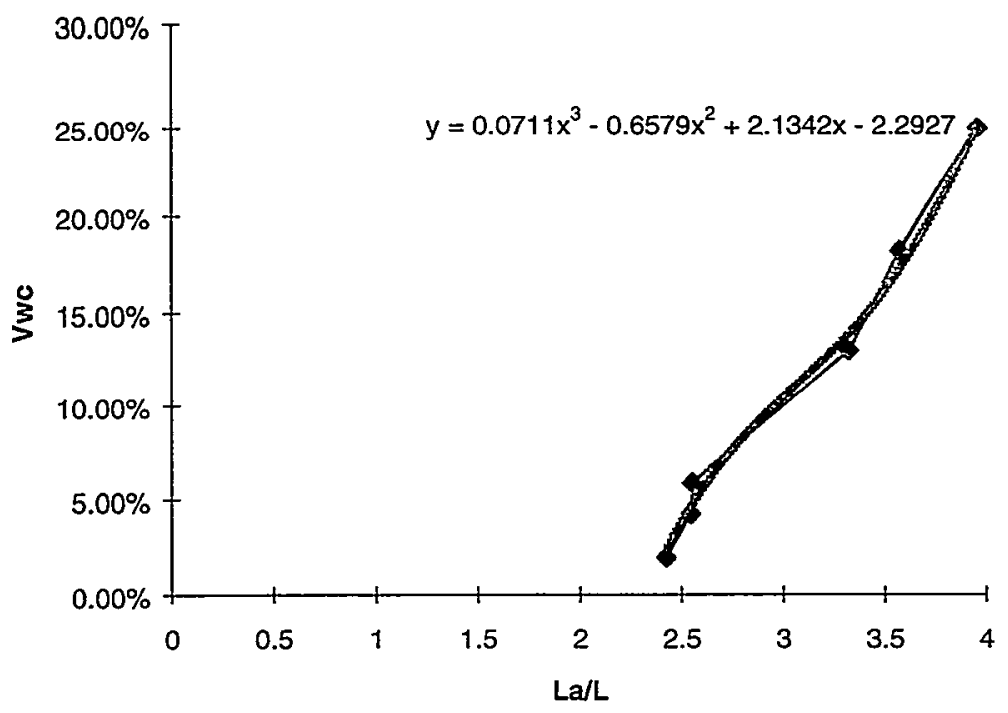

Figure G-12 


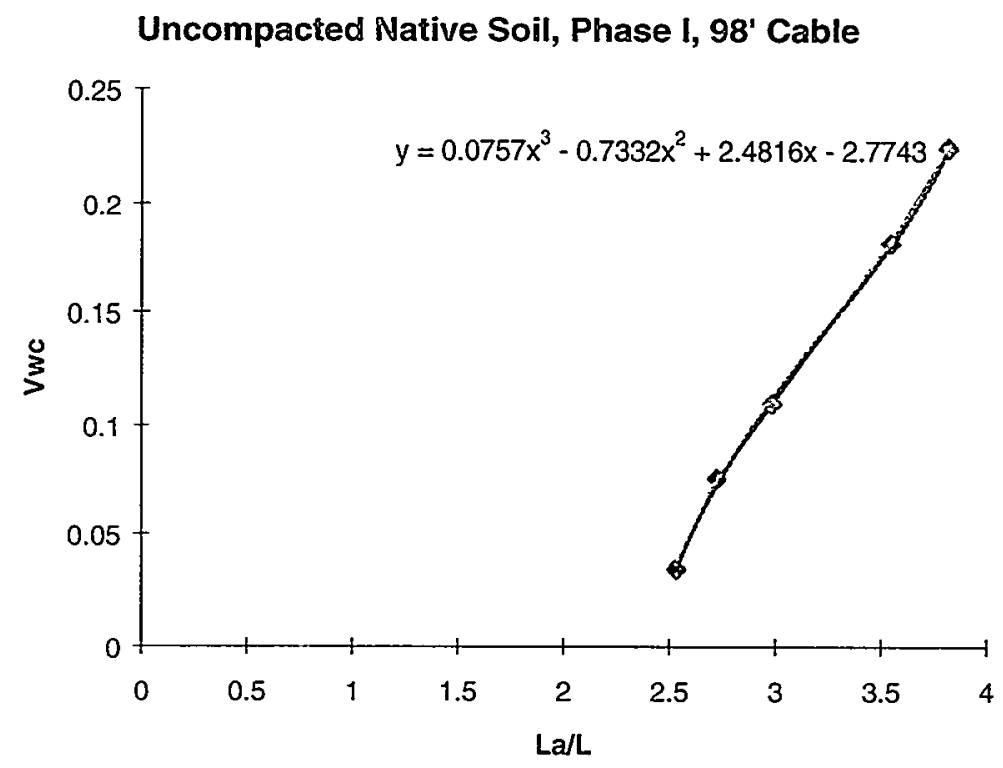

Figure G-13

\section{Uncompacted Native Soil, Phase I, 120' Cable}

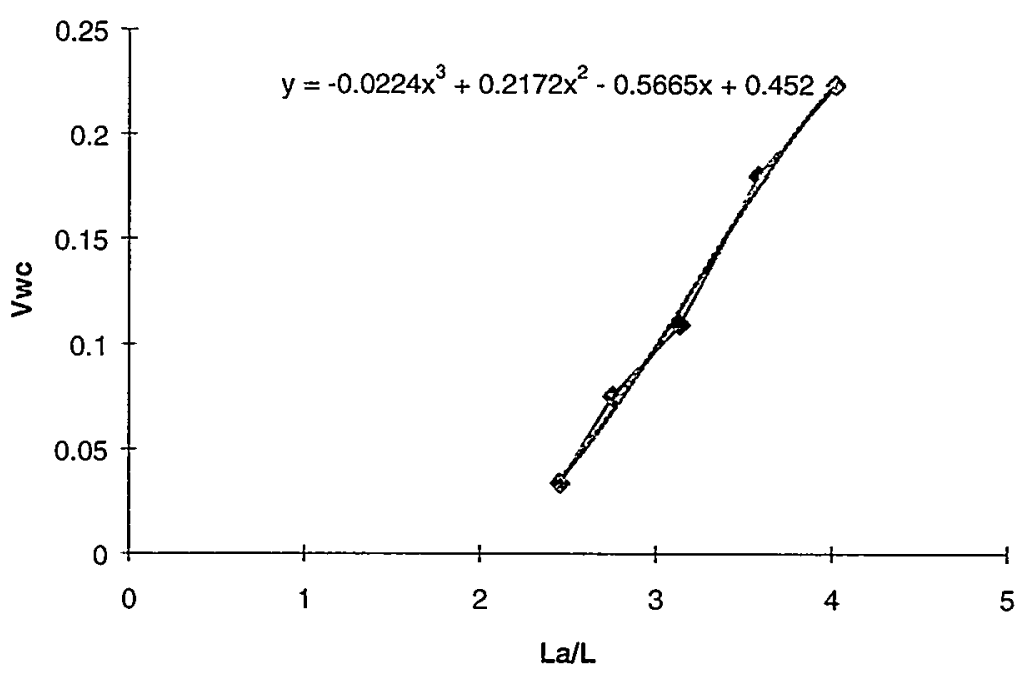

Figure G-14 
Uncompacted Native Soil, Phase I, 138' Cable

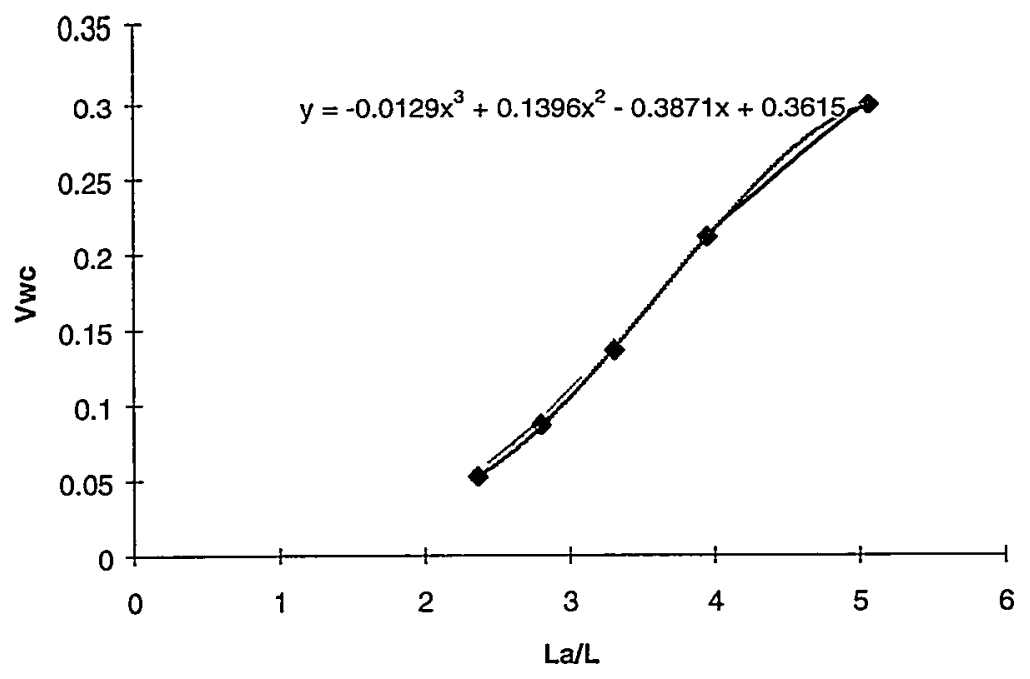

Figure G-15

Sand, Phase I, Short Cables

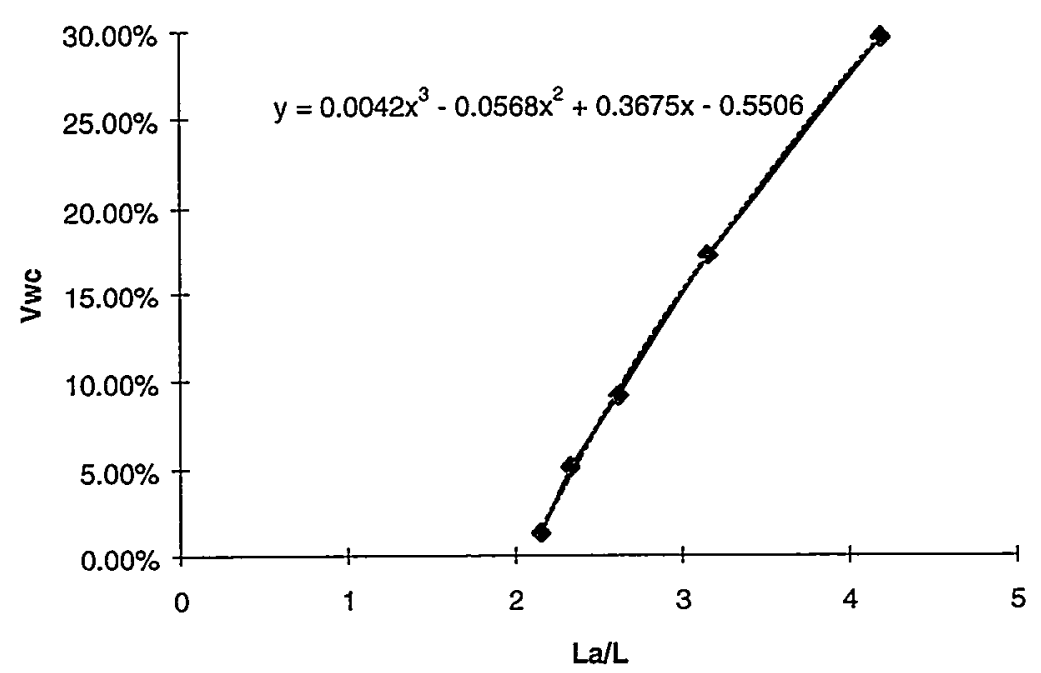

Figure G-16 


\section{Sand, Phase I, Long Cables}

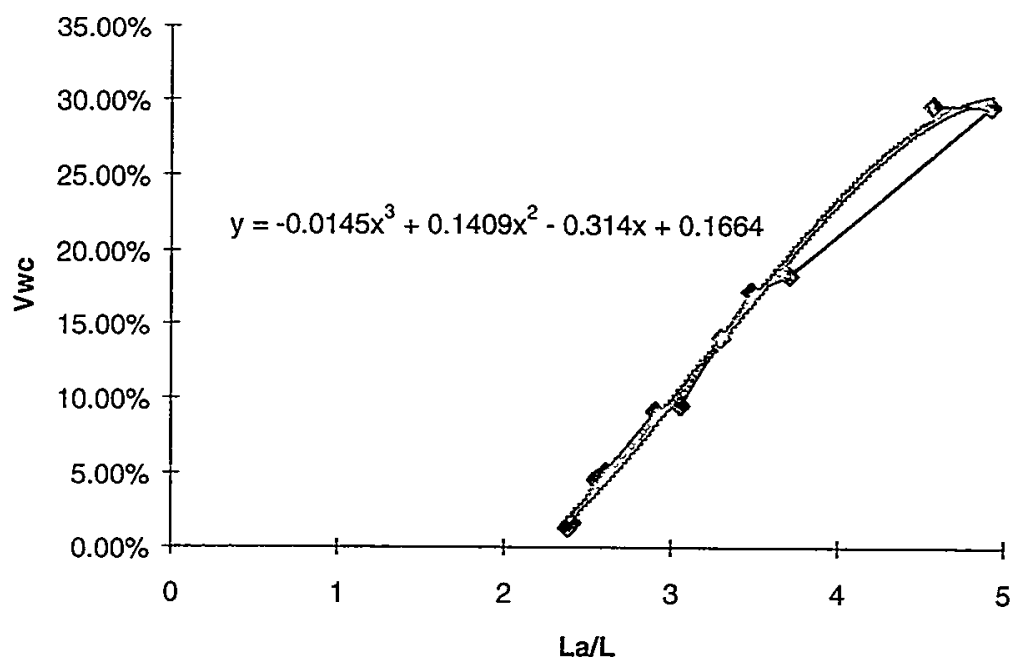

Figure G-17

Compacted Native Soil, Phase II, 85' Cable

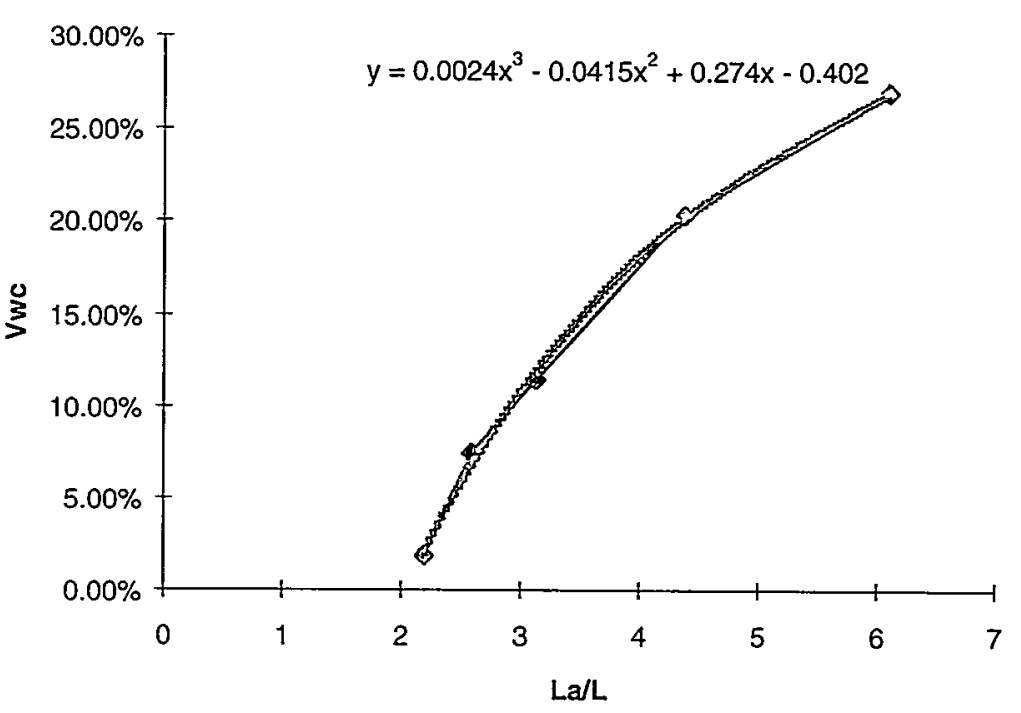

Figure G-18 
Compacted Native Soil, Phase II, 102' Cable

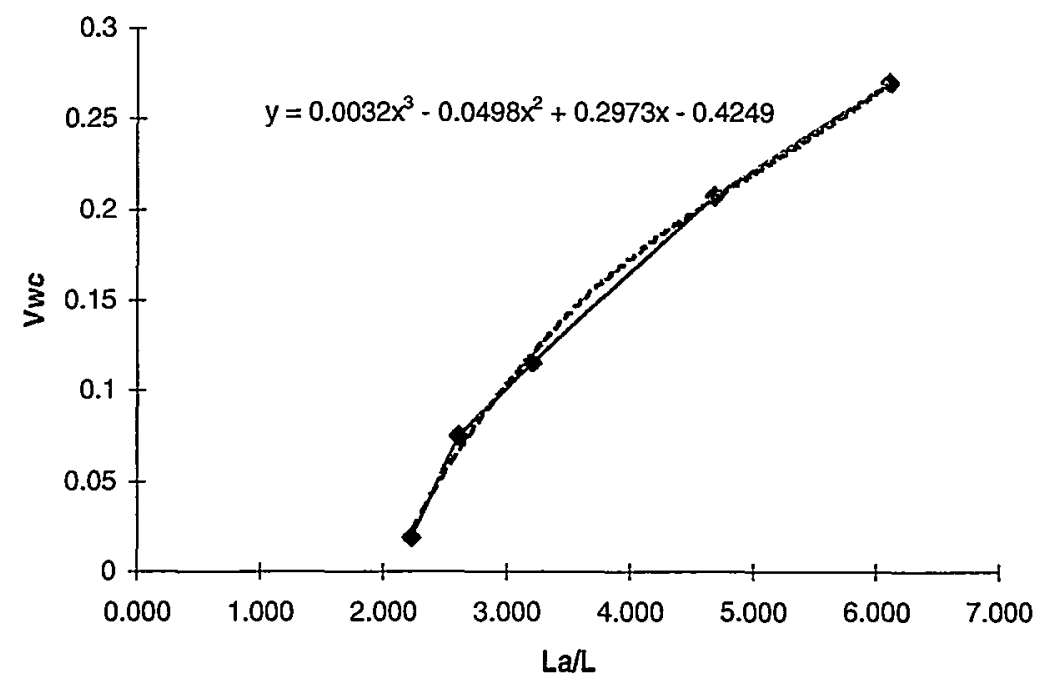

Figure G-19

Compacted Native Soil, Phase II, 119' Cable

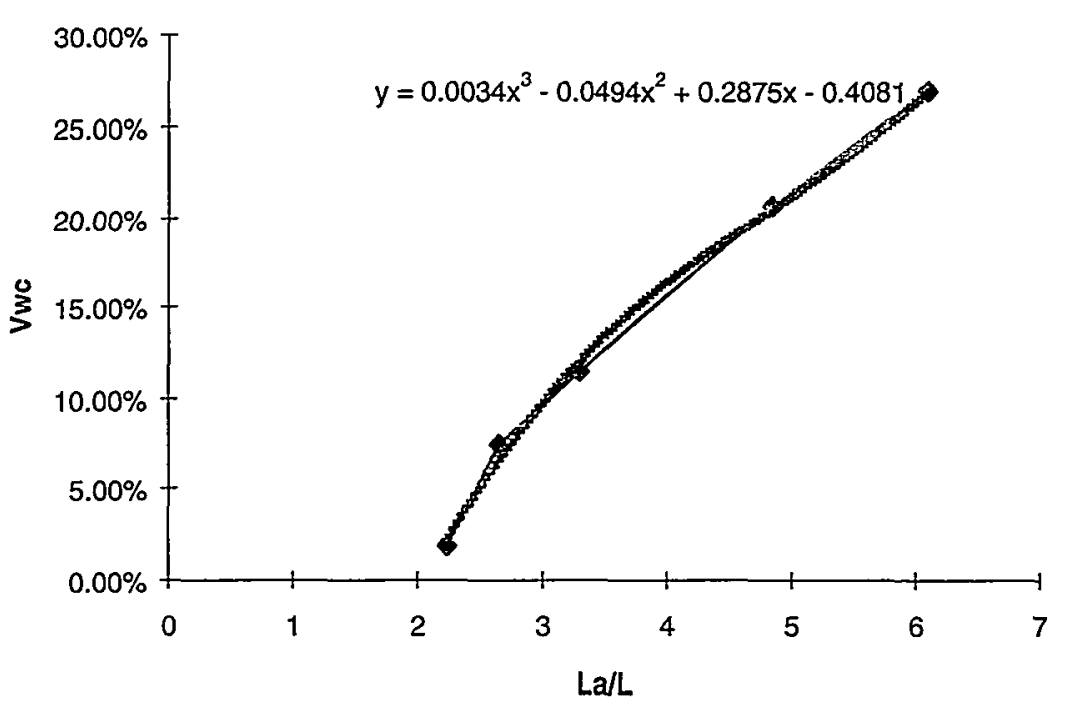

Figure G-20 


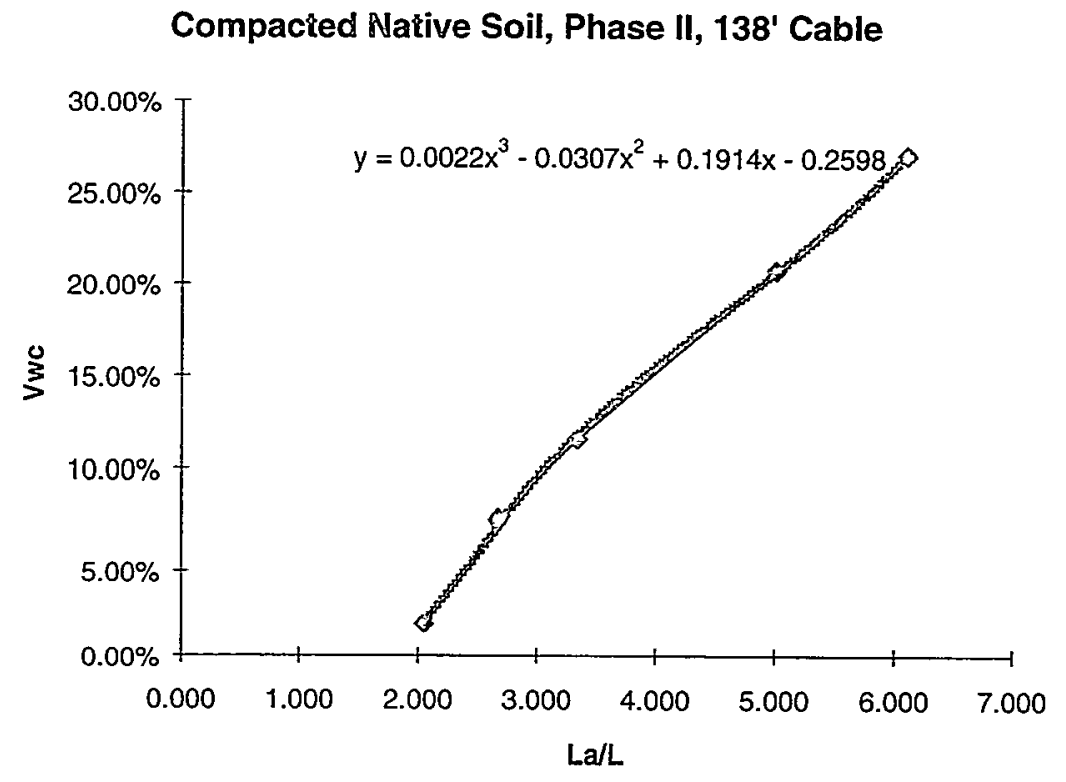

Figure G-21

Compacted Native Soil, Phase II, 156' Cable

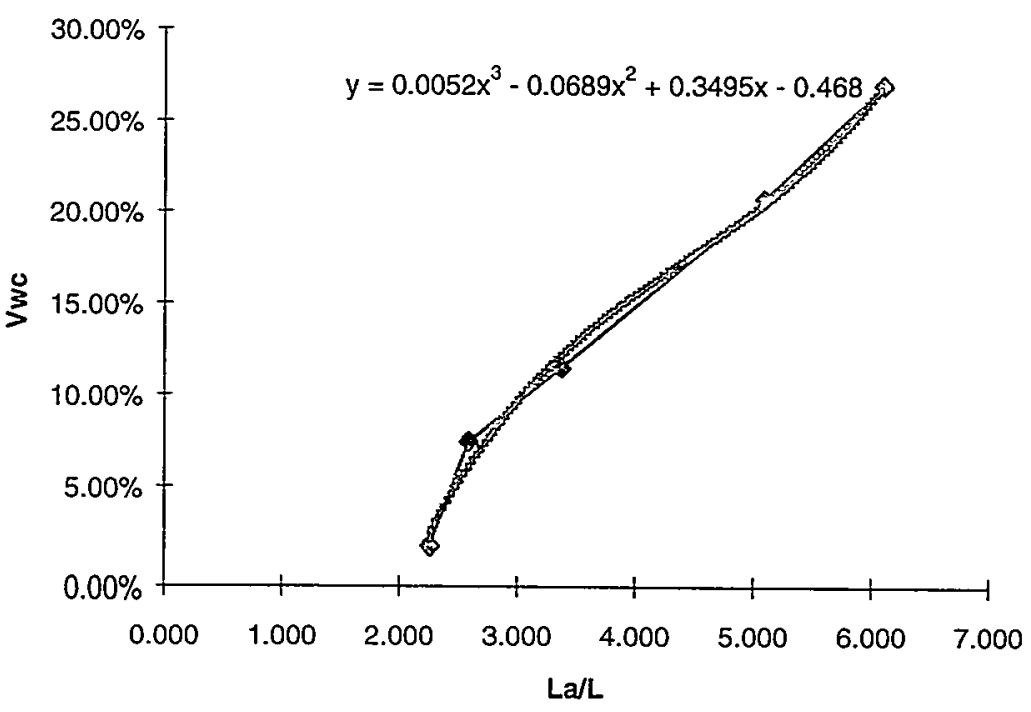

Figure G-22 
Uncompacted Native Soil, Phase II, $85^{\prime}$ Cable

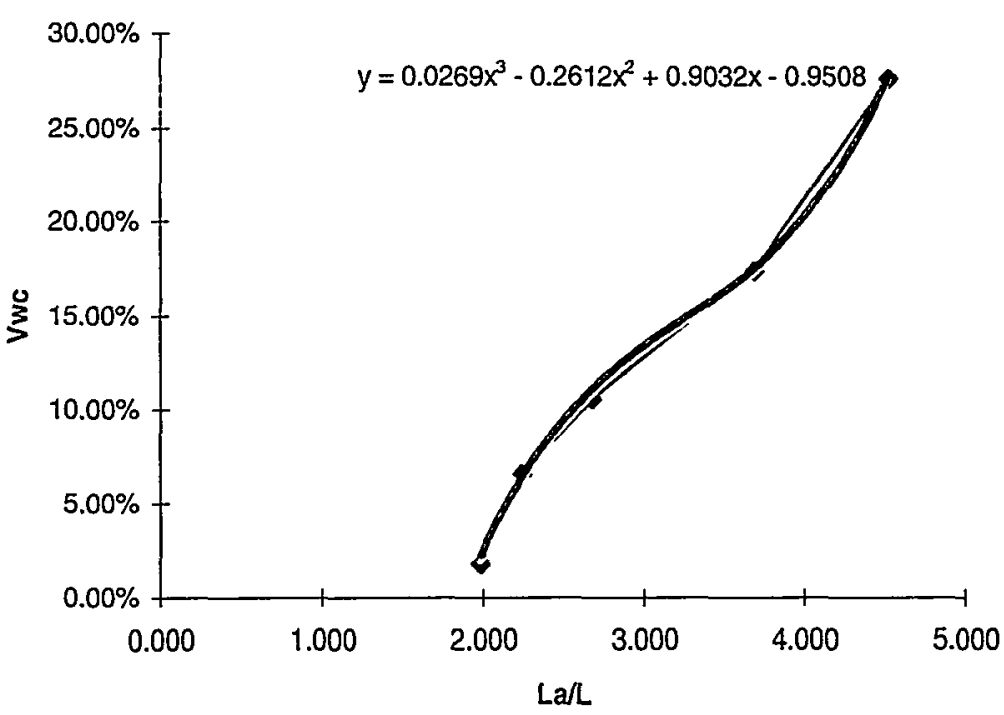

Figure G-23

Uncompacted Native Soil, Phase II, 102' Cable

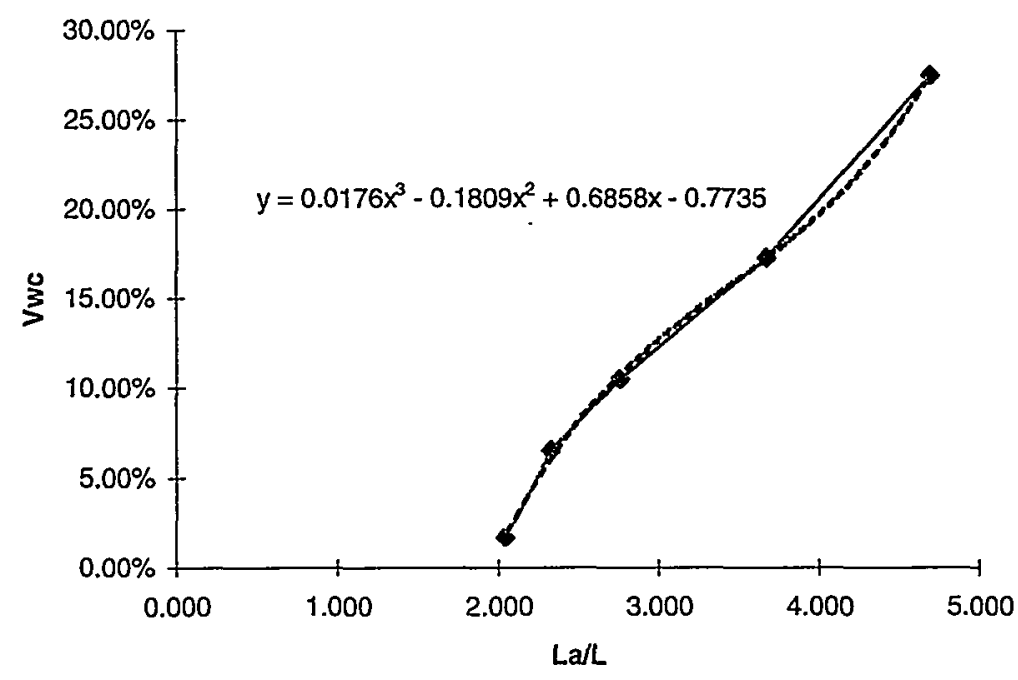

Figure G-24 
Uncompacted Native Soil, Phase II, 119' Cable

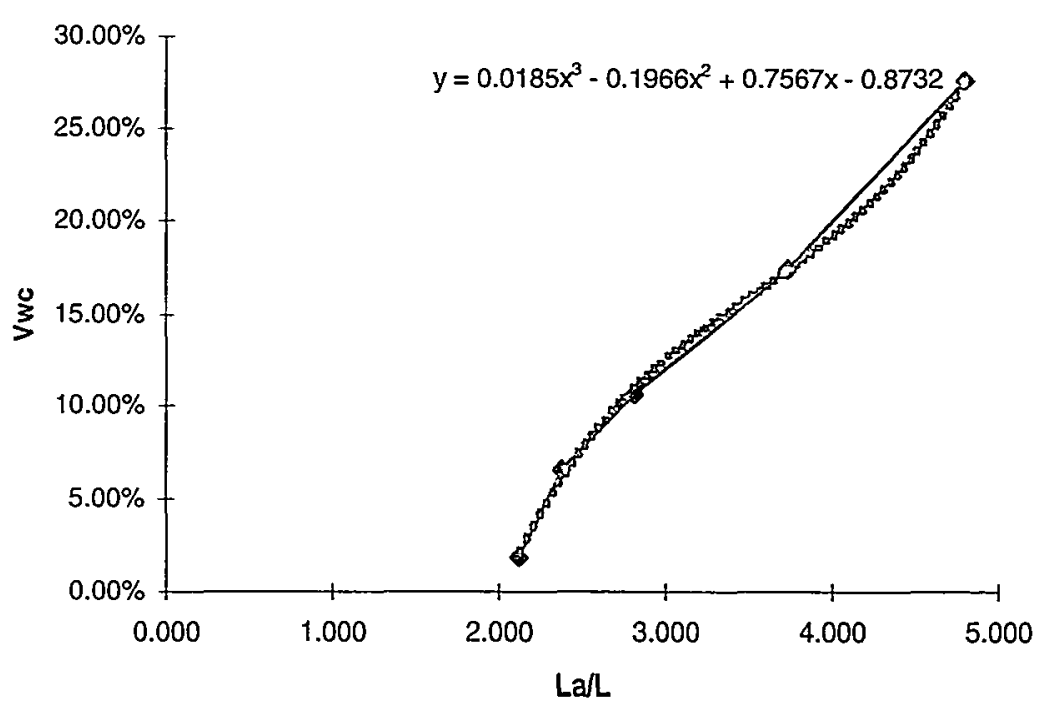

Figure G-25

\section{Uncompacted Native Soil, Phase II, 138' Cable}

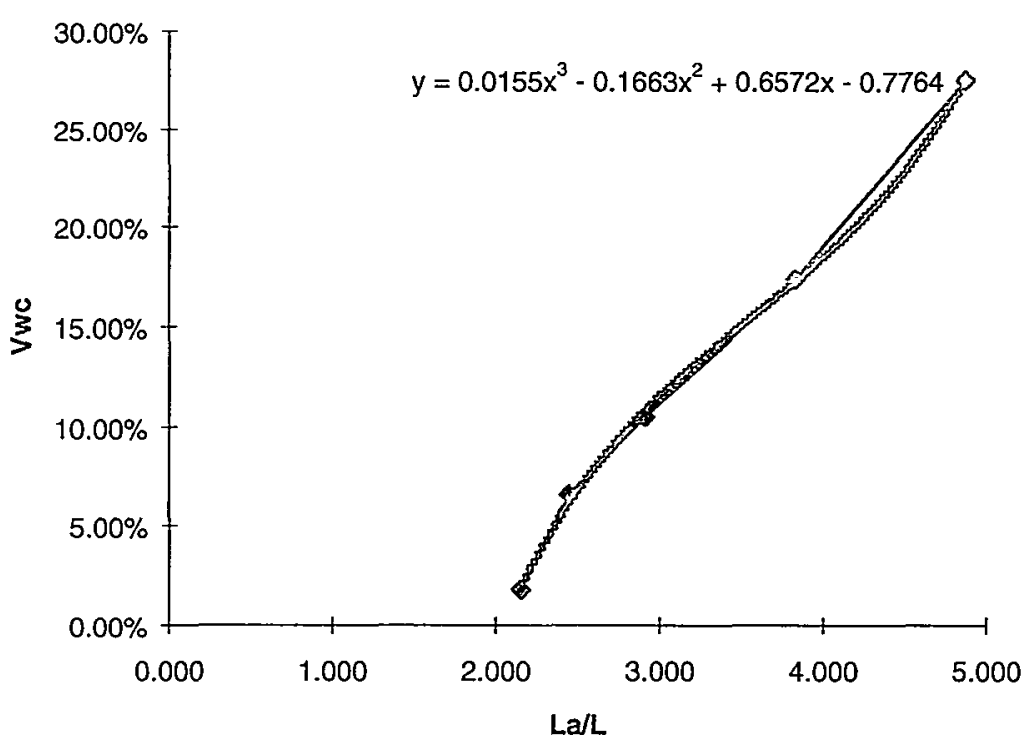

Figure G-26 
Uncompacted Native Soil, Phase II, 156' Cable

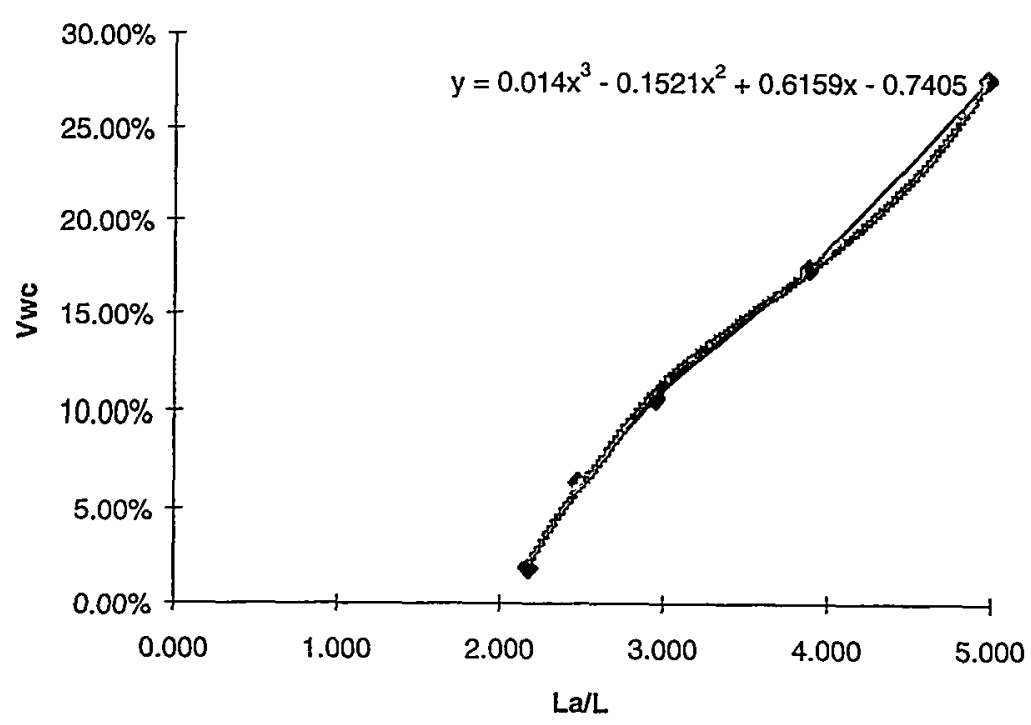

Figure G-27

Sand, Phase II, All Cable Lengths

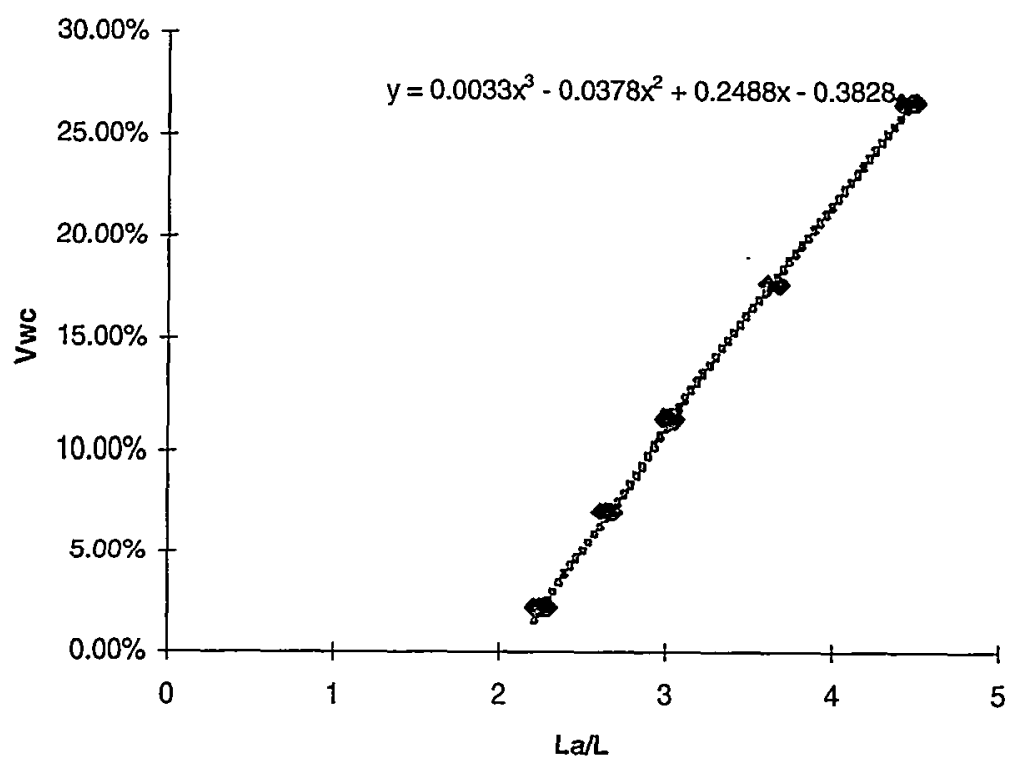

Figure G-28 


\section{ATTACHMENT H}

Surface Runoff System Calibration 


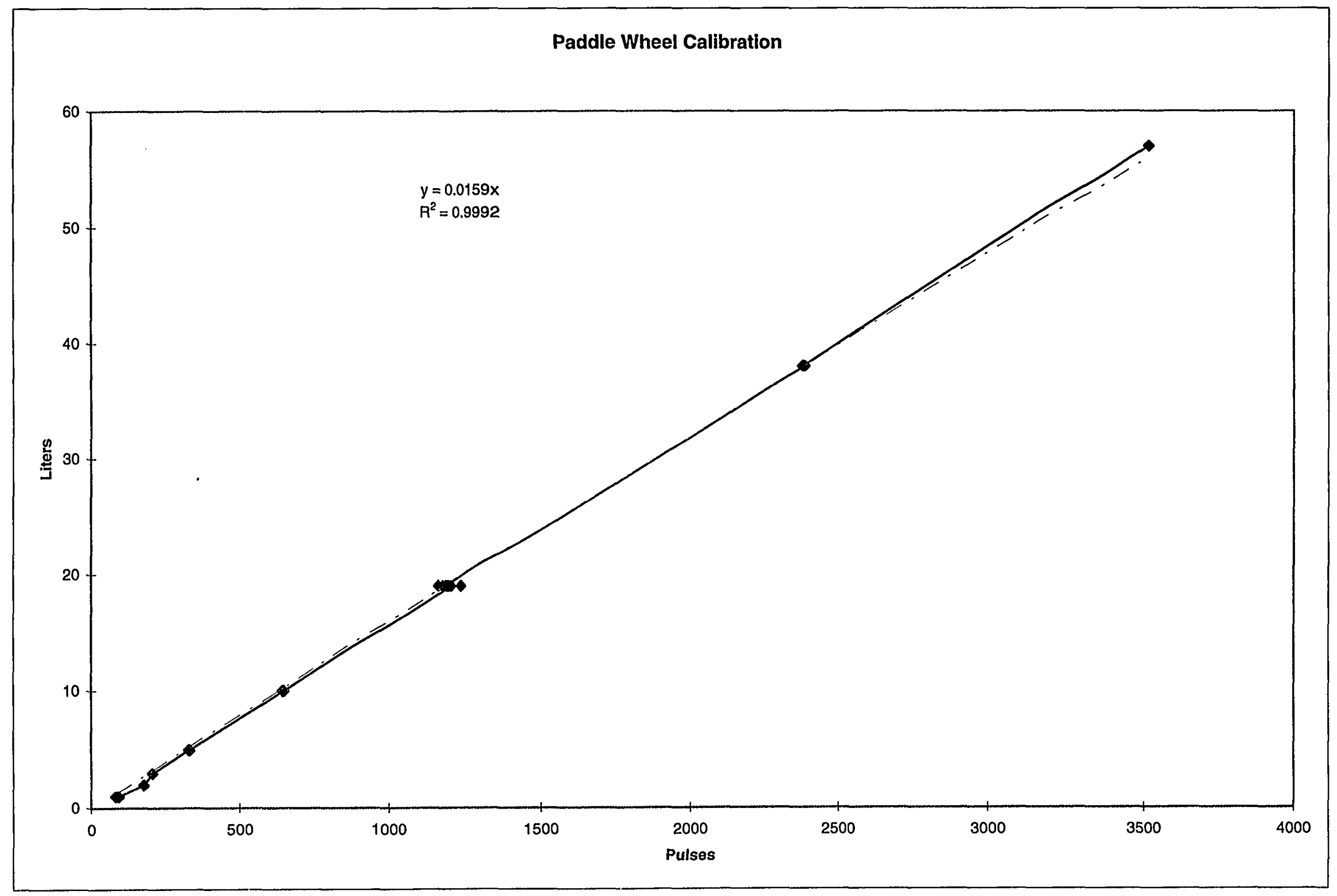




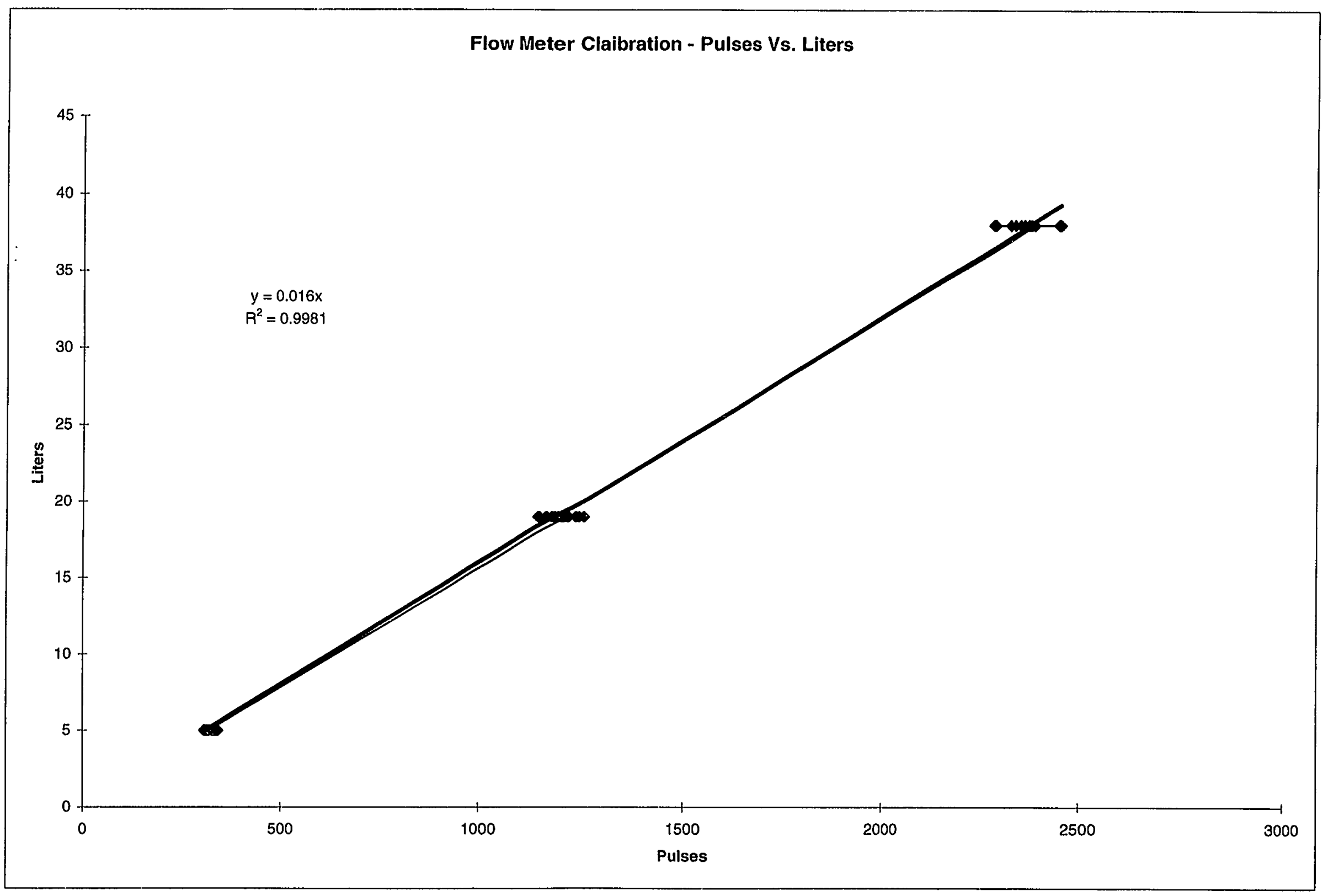




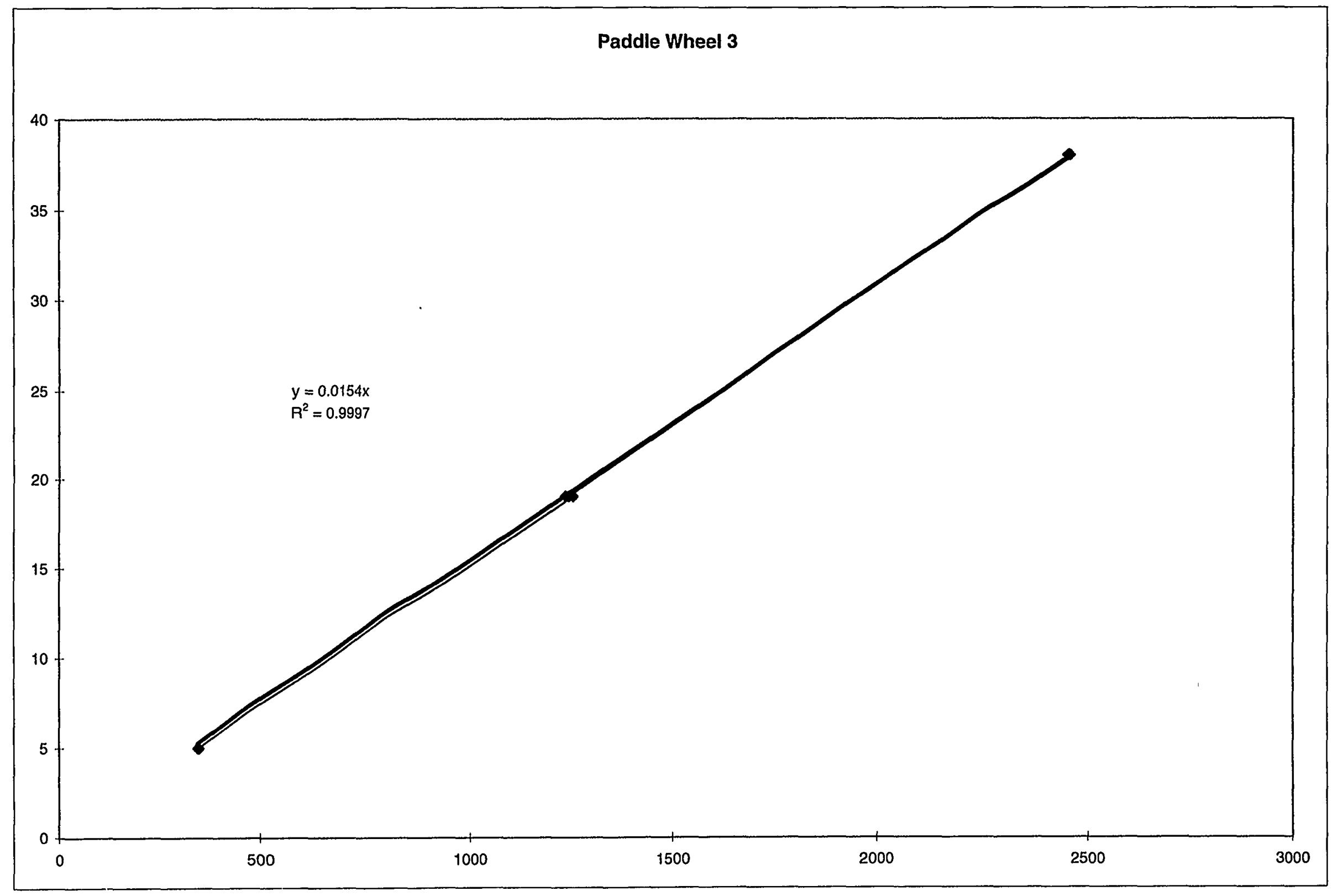




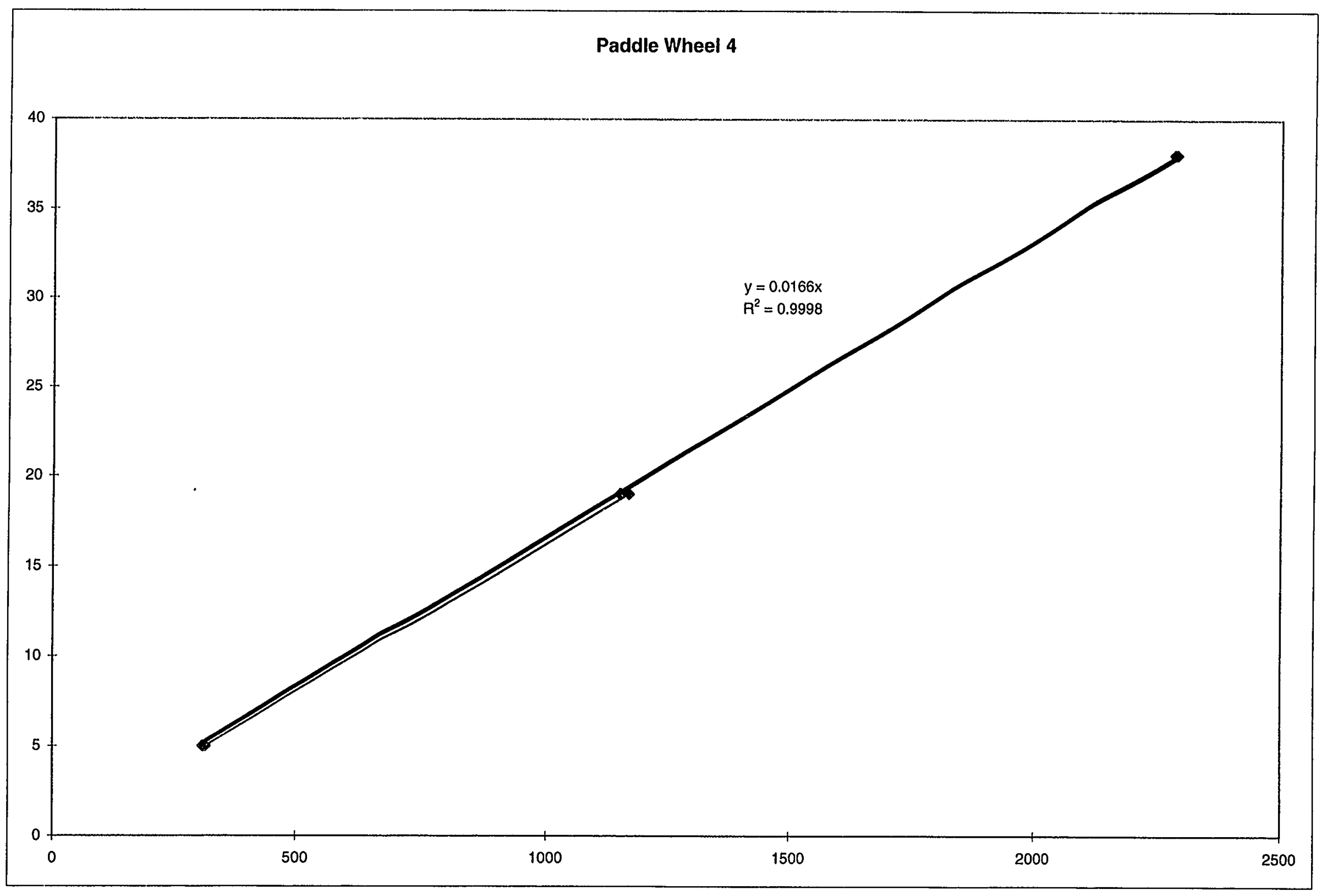




\section{DISTRIBUTION}

1 Bureau of Land Management

Attn: Brent Lewis

Denver Service Center

Building 50

Denver, CO 80225

1 Bureau of Land Management

Attn: Mike Moran

U.S. Department of Interior

Las Vegas Field Office

4765 Vegas Drive

Las Vegas, NV 89108

1 Bureau of Land Management

Attn: Stephanie Odell

Farmington District Office

1235 La Plata Highway

Farmington, NM 87401

1 CETCO

Attn: Jim Olsha

$1350 \mathrm{~W}$. Shure Dr.

Arlington Heights, IL 60004

1 CH2M Hill

Attn: Michael Yacyshyn

3 Hutton Center Drive

Suite 200

Santa Ana, CA 92707

1 City of Tuscon

Attn: Chris Leverenz, Disposal Administrator

Solid Waste Management Department

P.O. Box 27210

Tuscon, AZ 85726

1 Colloid Environmental Technologies Company

Attn: Bradford H. Miller

25105 Bellota

Mission Viejo,CA 92692

1 Duke Engineering

Attn: Cindy Ardito

1650 University Blvd. N.E.

Albuquerque, NM 87102

1 Duke Engineering

Attn: Jim Studer

1650 University Blvd. N.E.

Albuquerque, NM 87102
1 Engineering Solutions \& Design

Attn: Jack P. Chappelle, P.E.

3916 Juan Tabo Blvd. N.E.

Albuquerque, NM 87111

1 Exxon Biomedical Sciences, Inc.

Attn: James H. Higinbotham

Mettlers Road

CN 2350

East Millstone, NJ 08875-2350

1 FERMCO

Attn: Larry Stebbins

MS: 81-2

P.O. Box 538704

Cincinnati, $\mathrm{OH} 45235$

1 Issa Hamud

P.O. Box 527

255 N. Main

Logan, UT 84321

1 Los Alamos National Laboratory

Attn: Bruce Erdal

EM-TD

MS J591

Los Alamos, NM 87545

1 MDM/Lamb. Inc.

Attn: Eric Rogoff

6121 Indian School Rd., N.E.

Suite 105

Albuquerque, NM 87110

1 National Research Council

Attn: Robert Andrews, Ph.D.

National Academy of Science

Board of Radioactive Waste Mgmt.

2001 Wisconsin Ave., N.W.

Washington, DC 20007

1 New Mexico Junior College

Attn: Pannell Library

Lovington Highway

Hobbs, NM 88240

1 New Mexico Environment Department

Attn: J. Espinosa

1190 St. Francis Drive

Santa Fe, NM 87503

1 New Mexico Environment Department

Attn: S. DinWiddie

1190 St. Francis Drive

Santa Fe, NM 87503

Dist-1 
1 New Mexico State Library 325 Don Gaspar

Santa Fe, NM 87503

1 New Mexico Tech

Attn: Martin Speere Memorial Library

Campus Street

Socorro, NM 87810

1 Oak Ridge National Laboratory

Attn: Tom Early

P.O. Box 2008

Oak Ridge, TN 37831

1 SCS Engineers

Attn: Jian W. (Frank) Lliu

2702 North $44^{\text {th }}$ Street

Suite 105B

Phoenix, AZ 85008-1583

1 Sadat Associates

Attn: John Caputo

116 Village Blvd.

Princeton, NJ 08543

1 Science \& Engineering Assoc., Inc. Attn: Bill Lowry

3205 Richards Lane

Suite A

Santa Fe, NM 87505

1 The Snow Company Inc.

Attn: Tom Snow

P.O. Box 90670

Albuquerque, NM 87199

1 Thomas Branigan Library

Attn: D. Dresp

106 W. Hadley St.

Las Cruces, NM 88003

1 U.S. Department of Energy

Attn: Skip Chamberlain

EM-53

Cloverleaf Building

19901 Germantown Rd.

Germantown, MD 20874

1 U.S. Department of Energy

Attn: Michele DeGennaro

Environmental Measurements lab

201 Varick St., $5^{\text {th }}$ floor

New York, NY 10014-4811
1 U.S. Department of Energy

Attn: Gillian Eaton

Rocky Flats

Tech Site, Bldg. T124A

P.O. Box 928

Golden, CO 80402

1 U.S. Department of Energy Attn: John Geiger

Savannah River Operations Office

P.O. Box A

Aiken, SC 29802

$1 \quad$ U.S. Department of Energy

Attn: Dennis Green

Idaho Operations Office

850 Energy Drive

Idaho Falls, ID 83401

1 U.S. Department of Energy

Attn: Marvin Gross

Fernald Field Office

P.O. Box 538704

Cincinnati, $\mathrm{OH} 45253$

1 U.S. Department of Energy

Attn: Thomas Hicks

Savannah River Operations Office

Bldg. 703-46A

P.O. Box A

Aiken, SC 29802

1 U.S. Department of Energy

Attn: Gary Huffman

Rocky Flats Office

Highway $93^{\text {rd }} \&$ Cactus St.

Golden, CO 80402

1 U.S. Department of Energy

Attn: Sharon Johnson

Savannah River Operations Office

703 A, Rm. B202

Aiken, SC 29802

1 U.S. Department of Energy

Attn: Jeff Lenhert

Albuquerque Operations Office

P.O. Box 5400

Albuquerque, NM 87185

1 U.S. Department of Energy

Attn: Julianne Levings

Albuquerque Operations Office

P.O. Box 5400

Albuquerque, NM 87185 
1 U.S. Department of Energy

Attn: Jerry Lorenz

232 Energy Way

N. Las Vegas, NV 89030

1 U.S. Department of Energy

Attn: Scott McMullin

Savannah River Operations Office

P.O. Box A

Aiken, SC 29802

1 U.S. Department of Energy

Attn: David Roelant

232 Energy Way

N. Las Vegas, NV 89030

1 U.S. Department of Energy

Attn: Shannon Saget

Richland Operations Office-

P.O. Box 550, K8-50

Richland, WA 99352

1 U.S. Department of Energy

Attn: Pam Saxman

Albuquerque Operations Office

P.O. Box 5400

Albuquerque, NM 87185

1 U.S. Department of Energy

Dirk Schmidhofer

232 Energy Way

N. Las Vegas, NV 89030

1 U.S. Department of Energy

Attn: George Schneider

Idaho Operations Office

785 DOE Place

Idaho Falls, ID 83402

1 U.S. Department of Energy

Attn: Mel Shupe

Federal Energy Technology Center

Industrial Park

P.O. Box 3462

Butte, MT 59702

1 U.S. Department of Energy

Attn: Helen Stolz

Nevada

P.O. Box 98518

Las Vegas, NV 89193
1 U.S. Department of Energy

Attn: Jef Walker

EM-53

Cloverleaf Bldg.

19901 Germantown Rd.

Germantown, MD 20874

1 U. S. Department of Energy

Attn: Jody Wangh

Grand Junction Office

2597 B 3/4 Road

Grand Junction, CO 81503

1 U.S. Department of Energy

Attn: James Wright

Savannah River Operations Office

Bldg. 703-46A

P.O. Box A

Aiken, SC 29803

1 U.S. Environmental Protection Agency

Attn: Darryl Petker, P.E.

State of California

Integrated Waste Management Board

8800 Cal Center Drive

Sacramento, CA 95826

1 U.S. Environmental Protection Agency

Attn: Robert Shelnutt

75 Hawthorn Street. H-2-2

San Francisco, CA 94105-3901

1 U. S. Environmental Protection Agency Attn: Ken Skahn

(5202G)

401 M Street S.W.

Washington, D.C. 20460

1 U.S. Environmental Protection Agency

Attn: Art Palomares

Region 8

$99918^{\text {th }}$ Street

Suite 500 MC: 8EPR-F

Denver, CO 80202-2466

1 University of New Mexico

Attn: John Stormont

Dept. of Civil Engineering

Albuquerque, NM 87131

1 University of New Mexico

Attn: Bruce Thompson

Dept. of Civil Engineering

Albuquerque, NM 87131 
1 University of Texas at Austin

Attn: David Daniel

Dept. of Civil Engineering

Cockrell Hall

Room 9.102

Austin, TX 78712

1 University of Wisconsin

Attn: Craig Benson

Engineering Hall 2214

1415 Engineering Drive

Madison, WI 53706

1 Westinghouse Savannah River Company

Attn: Michael Serrato

Savannah River Technology Center

Environmental Sciences Section

P.O. Box 616/773-42A

Aiken, SC 29802

1 MS0701 L. E. Shephard, 6100

1 MS0719 G. C. Allen, 6131

1 MS0719 T. D. Burford, 6131

10 MS0719 S.F. Dwyer, 6131

1 MS0719 D.A. Padilla, 6131

1 MS0724 J. B. Woodard, 6000

1 MS1132 W. B. Cox, 6100

1 MS1132 R. E. Fate, 6132

1 MS1147 F. B. Nimick, 6133

1 MS1148 G. L. Peace,

2 MS0619 Review \& Approval Desk 12690, for DOE/OSTI

2 MS0899 Technical Library, 4916

1 MS9018 Central Technical Files, 8940-2 